\title{
Temporary Losses of Highway Capacity and Impacts on Performance: Phase 2
}

November 2004

Prepared by

S. M. Chin

O. Franzese

D. L. Greene

H. L. Hwang

Oak Ridge National Laboratory

Oak Ridge, Tennessee

R. C. Gibson

The University of Tennessee

Knoxville, Tennessee 


\section{DOCUMENT AVAILABILITY}

Reports produced after January 1, 1996, are generally available free via the U.S. Department of Energy (DOE) Information Bridge:

Web site: http://www.osti.gov/bridge

Reports produced before January 1, 1996, may be purchased by members of the public from the following source:

National Technical Information Service

5285 Port Royal Road

Springfield, VA 22161

Telephone: 703-605-6000 (1-800-553-6847)

TDD: 703-487-4639

Fax: 703-605-6900

E-mail: info@ntis.fedworld.gov

Web site: http://www.ntis.gov/support/ordernowabout.htm

Reports are available to DOE employees, DOE contractors, Energy Technology Data Exchange (ETDE)

representatives, and International Nuclear Information System (INIS) representatives from the following source:

Office of Scientific and Technical Information

P.O. Box 62

Oak Ridge, TN 37831

Telephone: 865-576-8401

Fax: 865-576-5728

E-mail: reports@adonis.osti.gov

Web site: http://www.osti.gov/contact.html

This report was prepared as an account of work sponsored by an agency of the United States Government. Neither the United States government nor any agency thereof, nor any of their employees, makes any warranty, express or implied, or assumes any legal liability or responsibility for the accuracy, completeness, or usefulness of any information, apparatus, product, or process disclosed, or represents that its use would not infringe privately owned rights. Reference herein to any specific commercial product, process, or service by trade name, trademark, manufacturer, or otherwise, does not necessarily constitute or imply its endorsement, recommendation, or favoring by the United States Government or any agency thereof. The views and opinions of authors expressed herein do not necessarily state or reflect those of the United States Government or any agency thereof. 


\title{
TEMPORARY LOSSES OF HIGHWAY CAPACITY AND IMPACTS ON PERFORMANCE: PHASE 2
}

\author{
S.M. Chin \\ O. Franzese \\ D.L. Greene \\ H.L. Hwang \\ Oak Ridge National Laboratory \\ R.C. Gibson \\ The University of Tennessee
}

November 2004

\author{
Prepared by \\ OAK RIDGE NATIONAL LABORATORY \\ P.O. Box 2008 \\ Oak Ridge, Tennessee 37831-6285 \\ managed by \\ UT-Battelle, LLC \\ for the \\ U.S. DEPARTMENT OF ENERGY \\ under contract DE-AC05-00OR22725
}





\section{TABLE OF CONTENTS}

\section{Page}

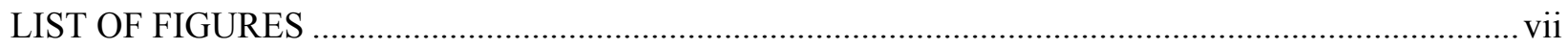

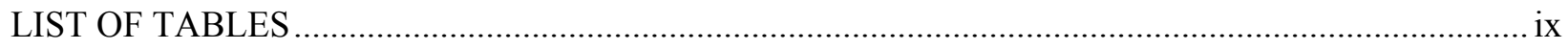

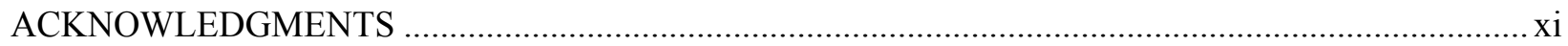

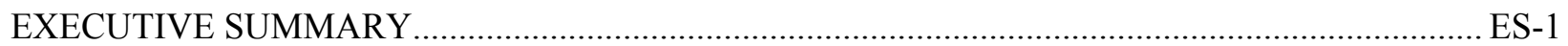

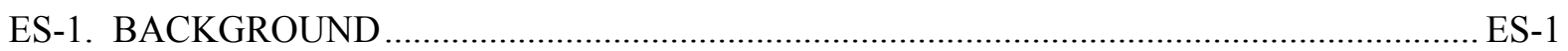

ES-2. OBJECTIVE AND SCOPE....................................................................................

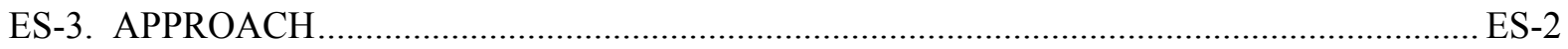

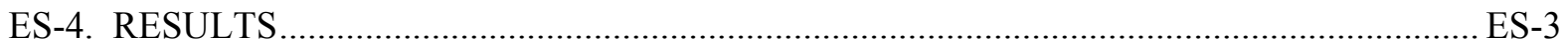

ES-5. COMPOSITE PICTURE AND NEXT STEPS ………………………………………..... ES-9

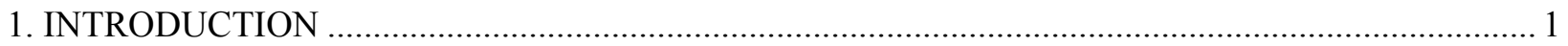

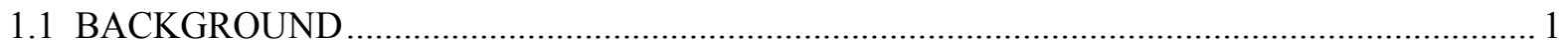

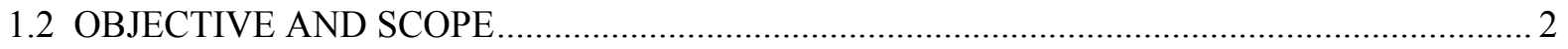

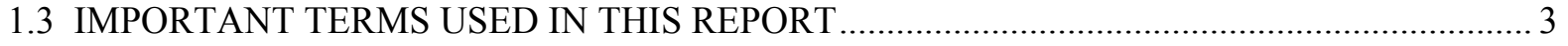

1.4 ORGANIZATION OF THE REPORT

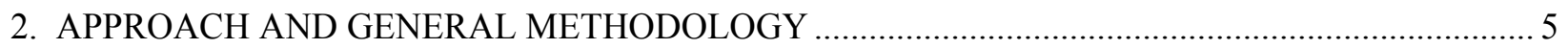

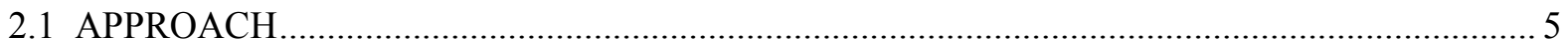

2.2 BASIC CONCEPTS OF CAPACITY, CAPACITY REDUCTION, AND DELAY ....................... 6

2.2.1 Determining Roadway Capacity ................................................................................

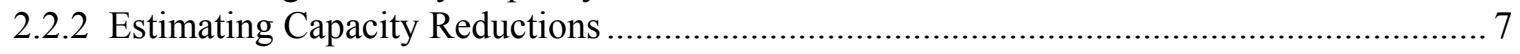

2.2.3 Estimating Delays for Localized Events ……………………………………………….... 11

2.3 DELAY IN THE CONTEXT OF TRAFFIC PATTERNS AND OTHER FACTORS.................. 14

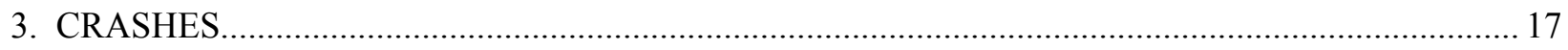

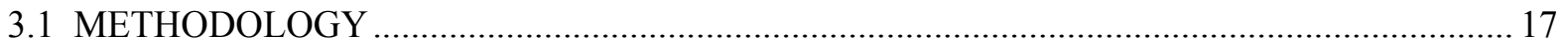

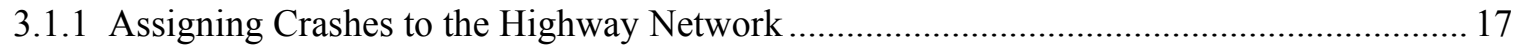

3.1.2 Estimating Capacity Reductions from Crashes.................................................................... 18

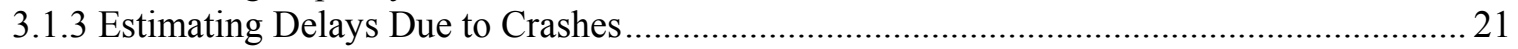

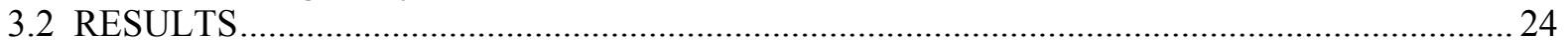

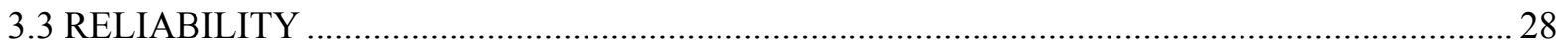

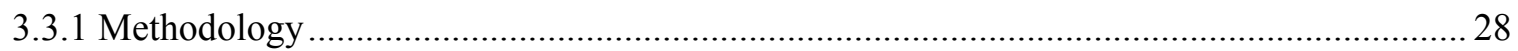

3.3.2 Data \& Key Assumptions ............................................................................................ 28

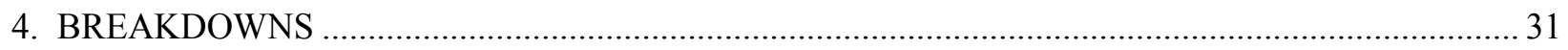

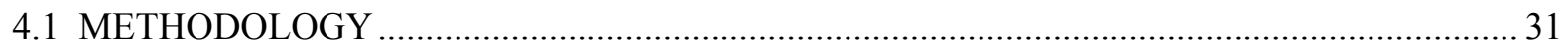

4.1.1 Estimating the Total Number of Breakdowns................................................................... 31

4.1.2 Assigning Each Breakdown to a Location in the Highway Network ...................................32

4.1.3 Assigning Temporal Characteristics to Each Breakdown.................................................... 32

4.1.4 Assigning Each Breakdown a Location within the Selected Link ……………………….....32

4.1.5 Estimating Capacity Reduction..................................................................................... 32 


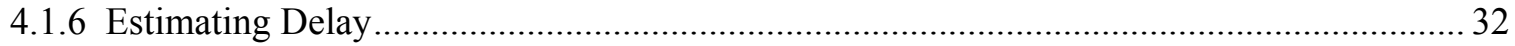

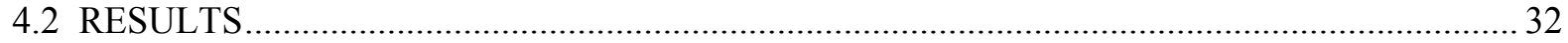

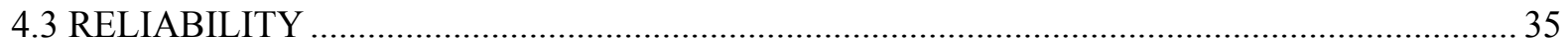

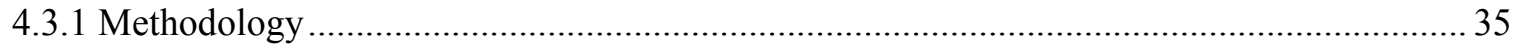

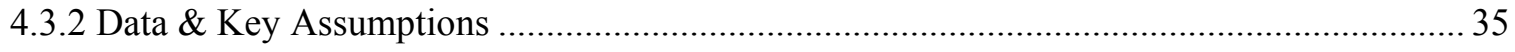

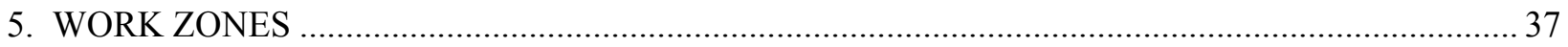

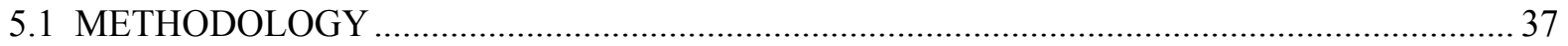

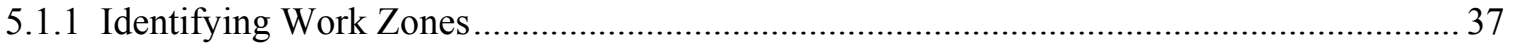

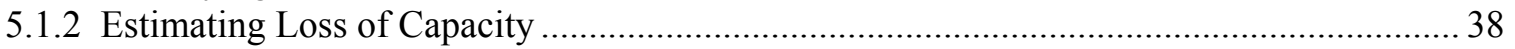

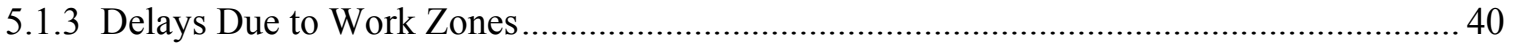

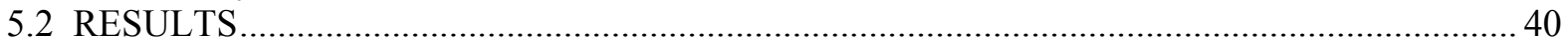

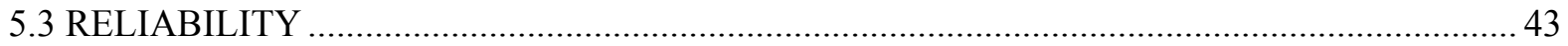

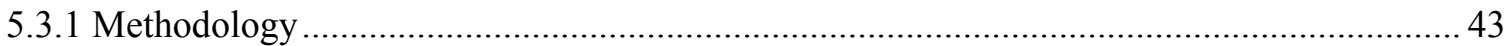

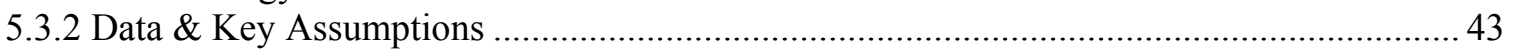

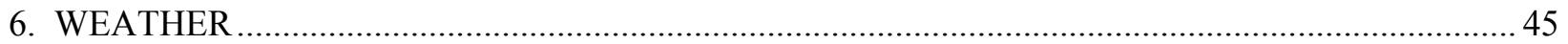

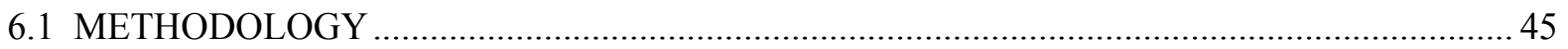

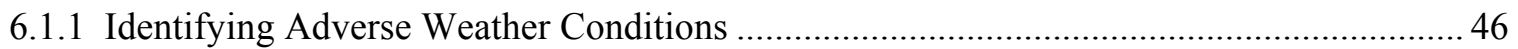

6.1.2 Mapping Adverse Weather Conditions to Highway Segments.......................................... 48

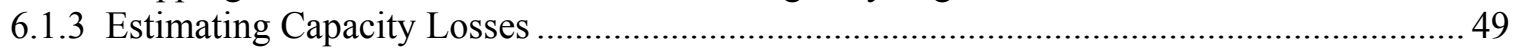

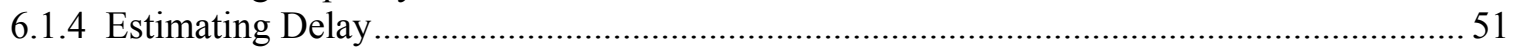

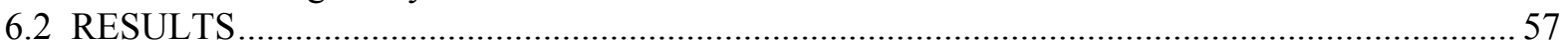

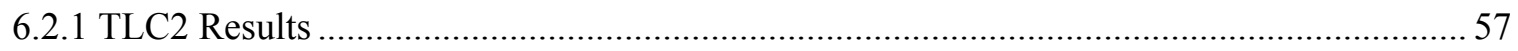

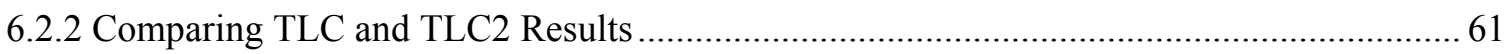

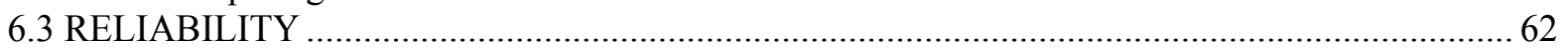

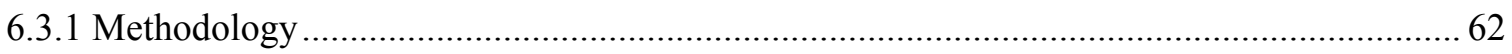

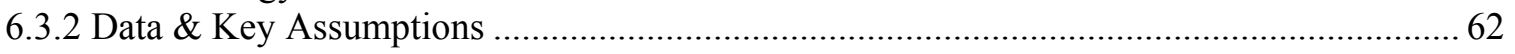

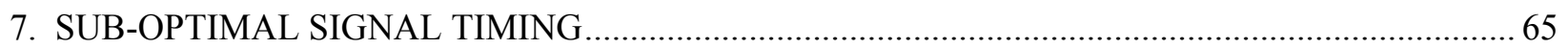

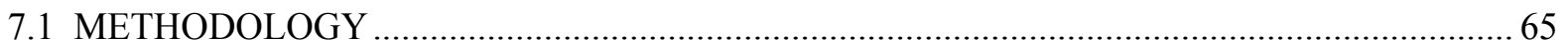

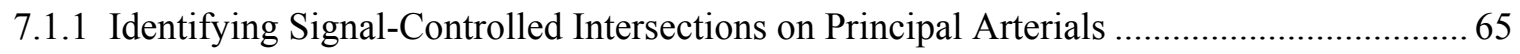

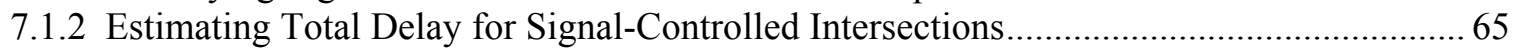

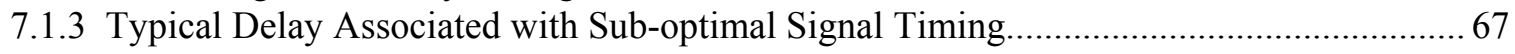

7.1.4 Typical Capacity Loss Associated with Sub-optimal Signal Timing .................................6 68

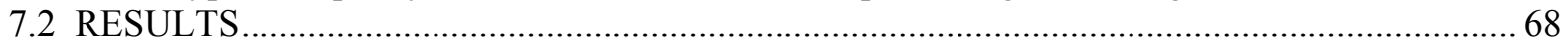

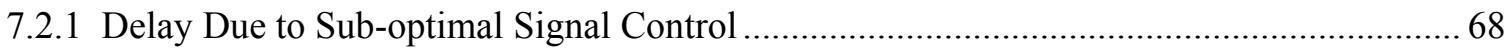

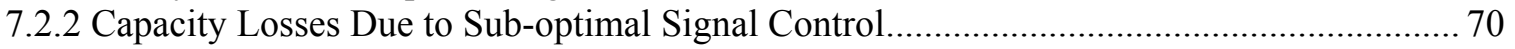

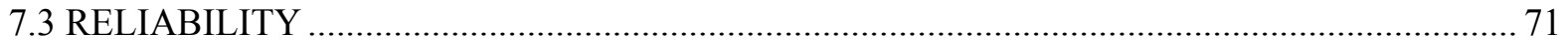

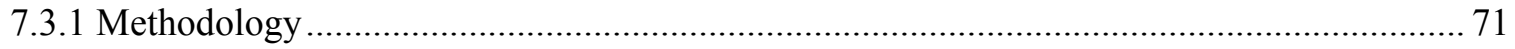

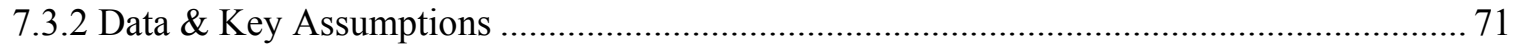

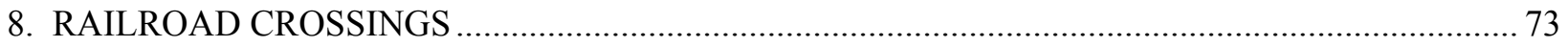

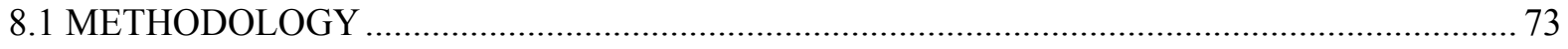

8.2 RESULTS

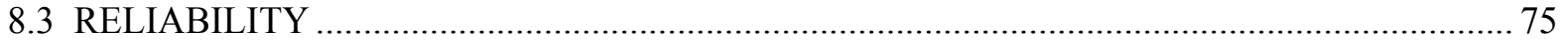

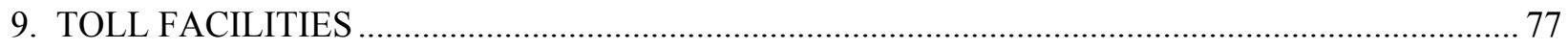

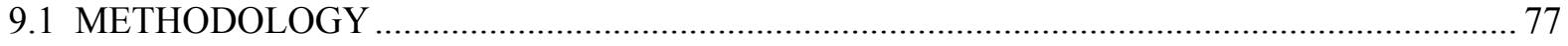

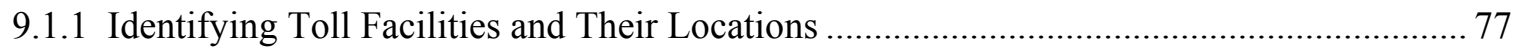

9.1.2 Determining the Number of Transactions at Each Facility ................................................ 77 


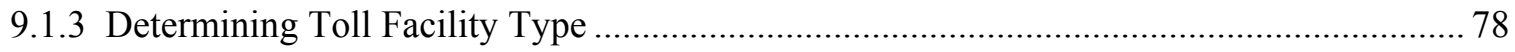

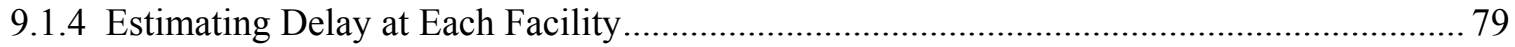

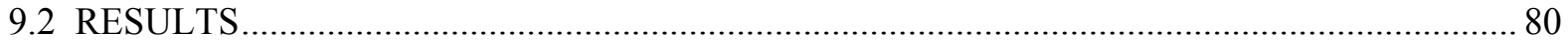

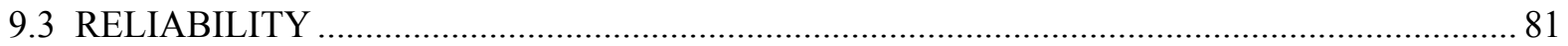

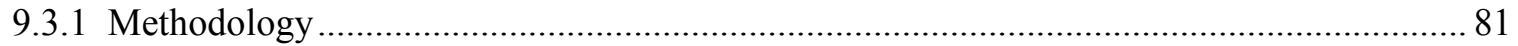

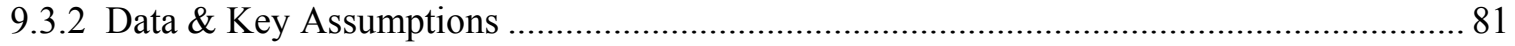

10. COMMERCIAL TRUCK PICKUP AND DELIVERY (PUD) ACTIVITIES IN URBAN AREAS.. 83

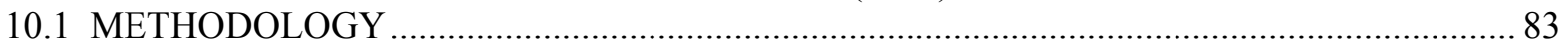

10.1.1 Identifying Arterial Links and Assigning Them to the Appropriate FHWA Urbanized

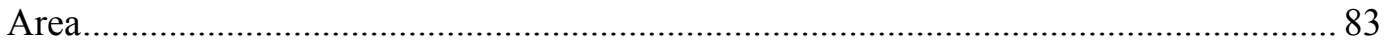

10.1.2 Estimating Weighted Average Capacity Reduction and Delay per PUD Event on Each Link for Each Land Use Type..................................................................................... 83

10.1.3 Estimating Weighted Average Delay per PUD Event for Each Urban Area for Each

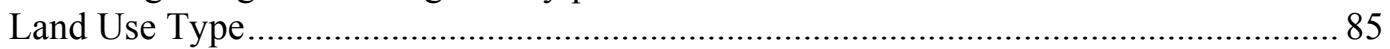

10.1.4 Estimating Annual Delay for Each Urban Area Based on Number of PUD Events.......... 85

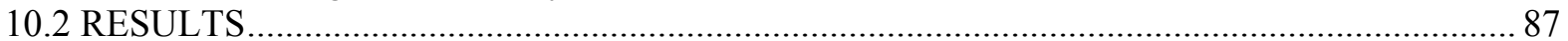

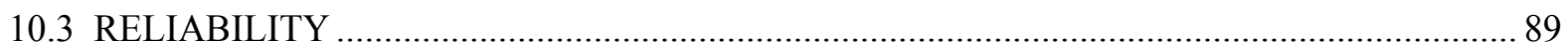

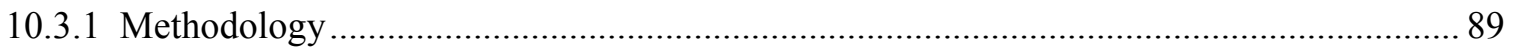

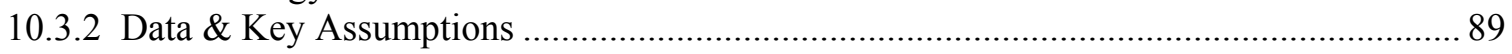

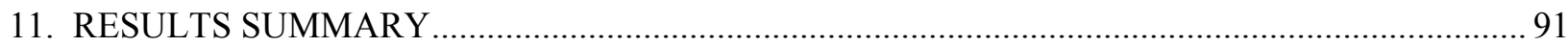

11.1 RESULTS

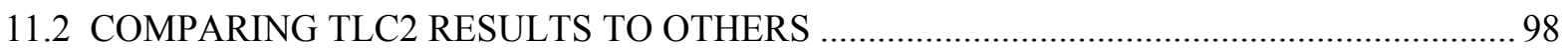

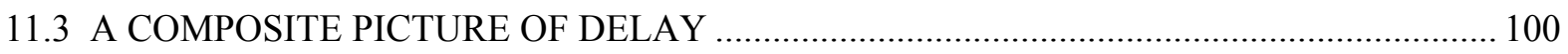

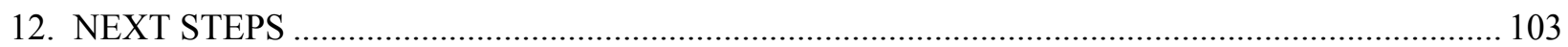

12.1 ESTIMATE IMPACTS FROM SIMULTANEOUS CAPACITY-REDUCING EVENTS ...... 103

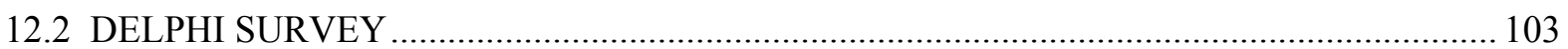

12.3 METHODOLOGY FOR ANALYZING DETAILED DELAY ESTIMATES ........................ 103

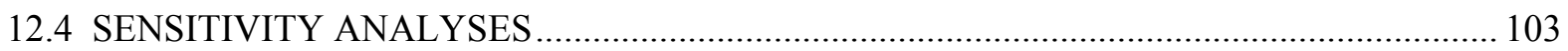

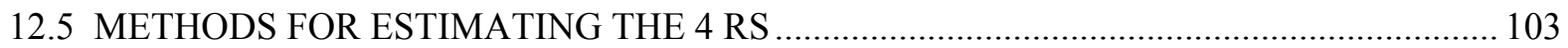

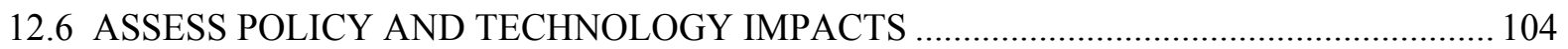

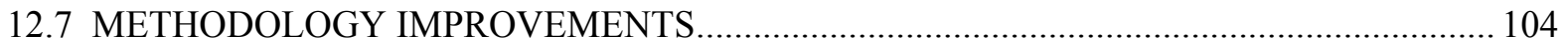

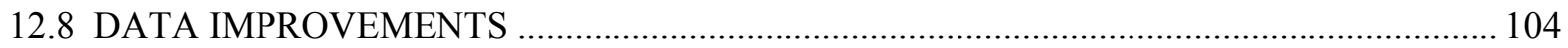

12.9 CONSIDER PUBLISHING TLC STUDY AS AN ANNUAL REPORT ............................... 104

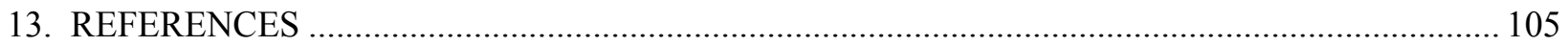




\section{LIST OF FIGURES}

ES-1 Most of the delay attributed to TLC events was caused by crashes and work zone activities

ES-2 A comparison of TLC2 estimates with those from two studies by TTI...

1 The highways within the scope of TLC2 accounted for about 54 percent of the VMT in the U.S. in 1999.

Analytical framework.

3 Flow rate vs. density for a highway segment with capacity $\mathrm{C}$ and a highway segment with capacity $\mathrm{C}^{\prime}=\alpha \mathrm{C}$

Flow rate vs. density for a highway segment

Demand Q1 equal to or less than bottleneck capacity $\mathrm{C}^{\prime}=\alpha \mathrm{C}$.

Demand Q1 larger than bottleneck capacity $C^{\prime}=\alpha C$

Cumulative flow vs. time for a highway bottleneck............................................................

8 Cumulative flow vs. time for a highway bottleneck with demand and capacity varying over time.

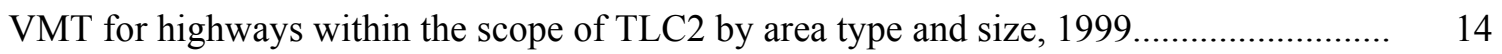

Traffic volume distributions for urban and rural areas.

Delay per crash by severity and highway type.

TLC2 estimates that most delay from breakdowns is experienced on urban principal arterials and rural principal arterials

TLC2 estimates that 98 percent of delay from work zones was experienced on freeways....

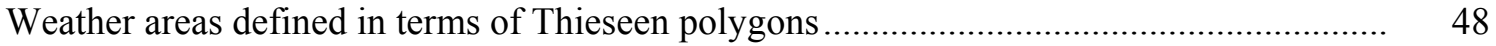

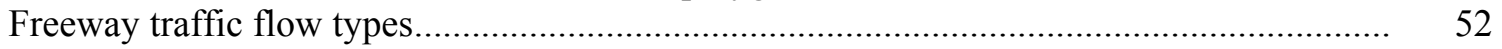

Speed-flow relationships for a typical basic freeway segment ...................................... 52

Speed relationship to volume/capacity ratio for basic freeway segment.............................. 53

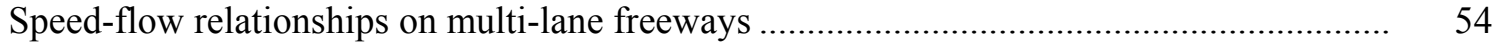

Speed relationship to volume/capacity ratio for multi-lane freeways ................................. 55

Most weather-related capacity reduction occurred from rain on urban and rural arterials .... 57

Most weather-related delay was experienced in urban areas ............................................. 58

Capacity reductions due to fog and snow estimated by TLC 2 were significantly lower than those estimated by the initial TLC study.....

Delays due to fog and snow estimated by TLC2 were significantly lower than those estimated by the initial TLC study.....

Delay from sub-optimal signal timing was greatest in very large urban areas........................ $\quad 70$

Nearly half of the delay from highway-rail at-grade crossings was experienced in small urban areas.

Over three-quarters of the delay from toll facilities in 1999 occurred in lanes using non-electronic collection methods.

Nearly 90 percent of the delay from PUD activities in 1999 occurred in very large urban areas.

Most of the delay on urban principal arterials from PUD activities in 1999 occurred in off-peak hours

Non-fatal crashes and work zones account for over two-thirds of the delay from temporary losses of capacity. 
31 Medium-sized urban areas experienced the least amount of delay from TLC events in 1999 .

32 When the amount of travel in each area type/size is considered, the delay experienced in medium-sized urban areas is more in line with size (in terms of population).

33 Delay from TLC events was most prevalent on urban freeways

34 Most delay from TLC events occurred during off-peak periods

35 On a per-mile-of-travel basis, delay from TLC events was more likely to occur during peak periods on congested roadways.....

36 A comparison of three studies that estimate delay: TLC2, TTI's Urban Mobility Study (85 urbanized areas), and TTI's estimate for the FHWA Office of Operations (all urbanized areas)

37 TLC2 delay estimates are significantly larger than TTI's (all-urban-area study) for most urban area sizes

38 Very large urban areas had a somewhat greater share of recurring delay than other area types 


\section{LIST OF TABLES}

ES-1 Summary of capacity loss \& delay estimates for freeways and principal arterials, 1999 ...... ES-4

ES-2 Summaries of delay by area type \& size, highway type, and traffic period \& congestion level......

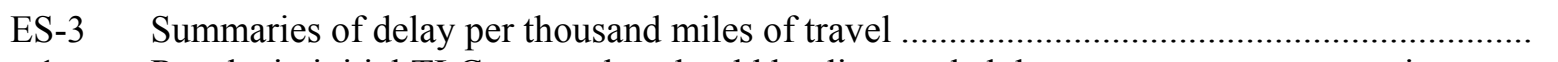

Results in initial TLC report that should be disregarded due to a computer processing error 2

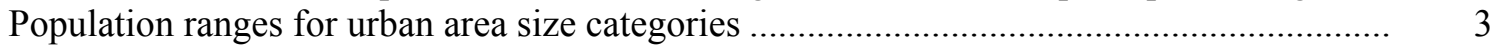

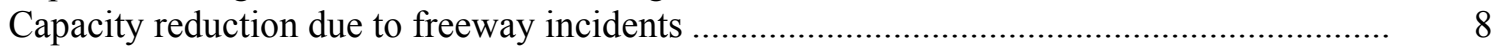

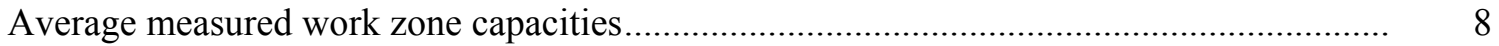

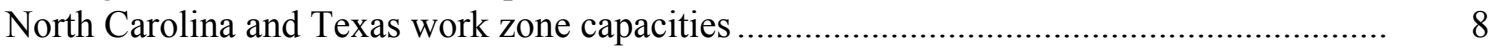

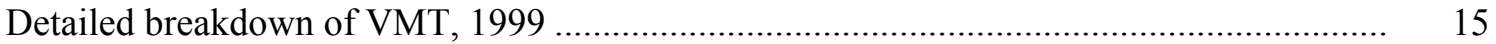

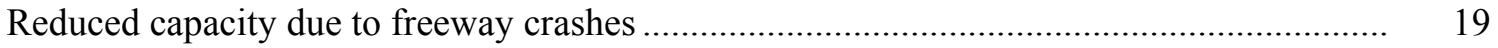

Probability of lane closures due to crashes and breakdowns ............................................. 20

Probability distribution of the number of lanes closed....................................................... 20

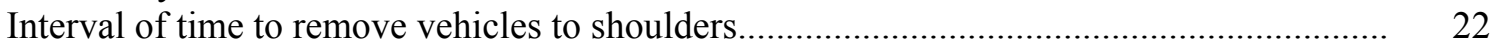

Estimated crash duration (in minutes): mean and standard deviation (SD) .......................... 23

Estimated crash duration (in minutes) ........................................................................... 23

13 Estimated capacity reductions and delay due to fatal and non-fatal crashes on freeways and

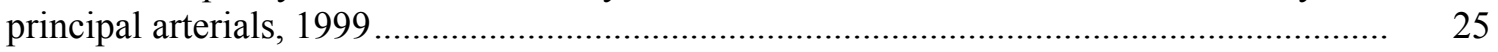

14 Capacity reductions \& delays from fatal crashes on freeways and principal arterials, 1999 . 26

15 Capacity reductions \& delay from non-fatal crashes on freeways and principal arterials, 1999

Estimated breakdowns and resulting capacity reduction and delay by highway type, 1999 . 33

Capacity reduction \& delay from breakdowns on freeways \& principal arterials ................. 34

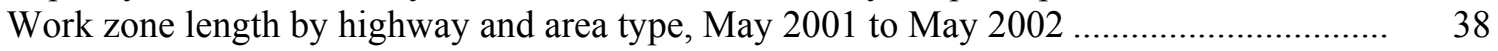

Capacities of open lanes at work zones........................................................................ 39

Probability distribution of the number of lanes closed in work zone area ............................ 40

Capacity reductions \& delay due to work zones .......................................................... 42

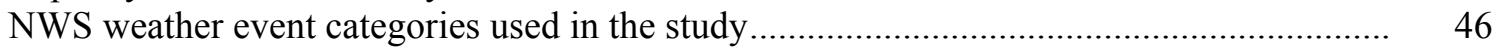

Mobility impacts of weather events ........................................................................ 50

Speed and capacity adjustment factors used in TLC2 ….................................................. 50

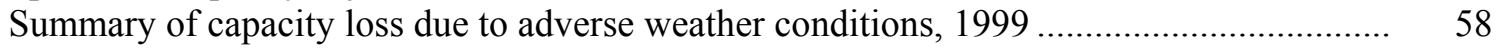

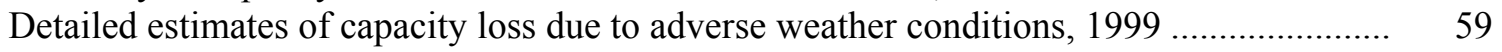

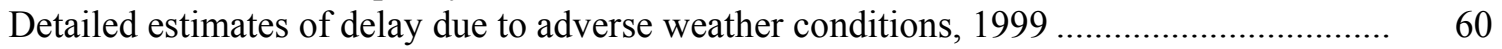

Capacity reduction \& delay from non-optimal signal timings on principal arterials ............ $\quad 69$

Capacity loss on principal arterials due to sub-optimal signal control, 1999........................ 71

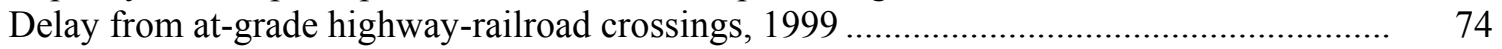

Per-vehicle delay assumptions by toll collection and delay type ......................................... 79

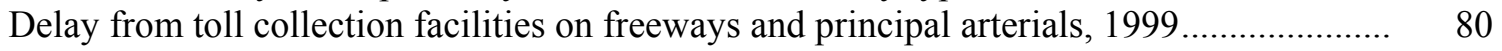

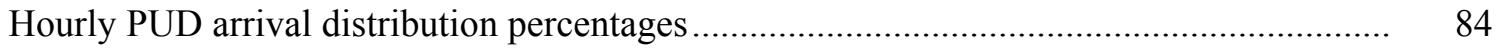

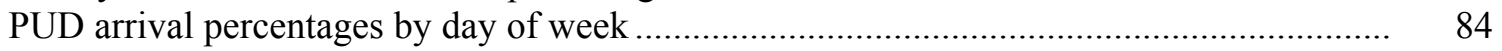

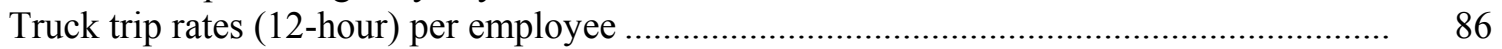

Capacity reduction and delay from PUD events, 1999 .................................................... 88

Summary of capacity loss \& delay estimates for freeways \& principal arterials ................. $\quad 92$

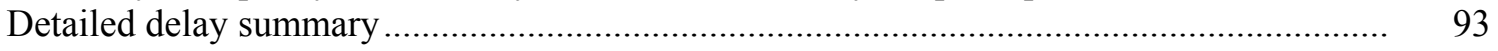

Summaries of delay by area type \& size, highway type, and traffic period \& congestion level...... 
41 Composite estimates of sources of delay 


\section{ACKNOWLEDGMENTS}

The authors gratefully acknowledge the support of the Office of Operations of the Federal Highway Administration, U.S. Department of Transportation. They also thank Dr. Rolf R. Schmitt, Mr. Vincent Pearce and Mr. Jeffrey Lindley for their guidance in the course of this work and review of early drafts of this report. The authors, however, are solely responsible for any remaining deficiencies or errors. 


\section{EXECUTIVE SUMMARY}

\section{ES-1. BACKGROUND}

Traffic congestion and its impacts significantly affect the nation's economic performance and the public's quality of life. In most urban areas, travel demand routinely exceeds highway capacity during peak periods. In addition, events such as crashes, vehicle breakdowns, work zones, adverse weather, railroad crossings, large trucks loading/unloading in urban areas, and other factors such as toll collection facilities and sub-optimal signal timing cause temporary capacity losses, often worsening the conditions on already congested highway networks. The impacts of these temporary capacity losses include delay, reduced mobility, and reduced reliability of the highway system. They can also cause drivers to re-route or reschedule trips. Such information is vital to formulating sound public policies for the highway infrastructure and its operation.

In response to this need, Oak Ridge National Laboratory, sponsored by the Federal Highway Administration (FHWA), made an initial attempt to provide nationwide estimates of the capacity losses and delay caused by temporary capacity-reducing events (Chin et al. 2002). This study, called the Temporary Loss of Capacity (TLC) study, estimated capacity loss and delay on freeways and principal arterials resulting from fatal and non-fatal crashes, vehicle breakdowns, and adverse weather, including snow, ice, and fog. In addition, it estimated capacity loss and delay caused by sub-optimal signal timing at intersections on principal arterials. It also included rough estimates of capacity loss and delay on Interstates due to highway construction and maintenance work zones. Capacity loss and delay were estimated for calendar year 1999, except for work zone estimates, which were estimated for May 2001 to May 2002 due to data availability limitations. Prior to the first phase of this study, which was completed in May of 2002, no nationwide estimates of temporary losses of highway capacity by type of capacityreducing event had been made.

This report describes the second phase of the TLC study (TLC2). TLC2 improves upon the first study by expanding the scope to include delays from rain, toll collection facilities, railroad crossings, and commercial truck pickup and delivery (PUD) activities in urban areas. It includes estimates of work zone capacity loss and delay for all freeways and principal arterials, rather than for Interstates only. It also includes improved estimates of delays caused by fog, snow, and ice, which are based on data not available during the initial phase of the study. Finally, computational errors involving crash and breakdown delay in the original TLC report are corrected.

\section{ES-2. OBJECTIVE AND SCOPE}

The TLC2 study develops estimates of highway capacity losses and delay caused by temporary capacityreducing events. The scope of TLC2 includes all urban and rural freeways and principal arterials in the nation's highway system for 1999-delays on minor arterials, collectors, and local roads are not included. The highways within the scope of TLC2 accounted for about 54 percent of the highway vehicle-miles of travel (VMT) in 1999.

TLC2 attempts to quantify the extent of temporary capacity losses due to the following events:

- Crashes (fatal and non-fatal)

- Breakdowns

- Work zones 
- Adverse weather (rain, snow, fog, ice)

- Sub-optimal signal timing (urban principal arterials only)

- Railroad crossings (urban and rural principal arterials)

- Toll collection facilities

- Commercial truck pickup and delivery (PUD) activities (urban principal arterials only)

These events can cause impacts such as capacity reduction, delays, trip rescheduling, rerouting, reduced mobility, and reduced reliability. This study focuses on the reduction of capacity and resulting delays caused by the temporary events mentioned above. Impacts other than capacity losses and delay, such as re-routing, re-scheduling, reduced mobility, and reduced reliability, are not covered in this phase of research.

It should also be noted that the study does not attempt to estimate capacity losses and delays due to events that occur simultaneously, such as a crash that takes place during a snowstorm or a breakdown that takes place in a work zone. Such coinciding events can often cause more capacity loss and delay than they might have cause if they had occurred separately. The interaction of capacity-reducing events is an area of interest and may be addressed in future research. However, due to time and funding constraints of the initial phases of the study, this interaction was not modeled.

Finally, due to funding and time constraints, a thorough evaluation of TLC and TLC2 results was not possible. Therefore, at the end of each chapter, a brief discussion of the limitations of the methodology, data, and assumptions used to generate the capacity loss and delay for that event type is provided. The potential effects of these limitations on the final estimates are also discussed.

\section{ES-3. APPROACH}

The study uses traffic engineering modeling methods, the best available data, and engineering judgment to derive estimates of capacity losses and delays. Because direct measurements are scarce and available data are generally incomplete, the validity of the estimates is dependent on the reasonableness of a number of critical assumptions. The philosophy followed is to rely on published peer-reviewed studies whenever possible and, when assumptions must be based solely on the researchers' judgment, to err on the side of underestimating losses of capacity and delay. There is one general exception to this rule, which is discussed below.

A critical distinction is made between the loss of capacity and its impacts. Capacity is a measure of potential: it describes the maximum sustainable throughput at a point on a highway. As such, it is independent of the highway's actual level of use. Impacts, however, depend not only on the loss of capacity, but on the volume of traffic on the highway at the time the loss occurs. A crash occurring on an Interstate highway in the middle of the night will cause far less delay than, but possibly the same loss of capacity as, the same crash occurring during rush hour. Delay is measured in vehicle-hours, which can be converted to person-hours by multiplying by a suitable vehicle occupancy. Capacity loss, on the other hand, is a loss of potential throughput (measured in vehicles per lane per hour), integrated over time and a length of roadway. While capacity for a given point on a highway at a given amount of time is measured in vehicles per lane per hour (vplph), the general methodology used in this study attempts to estimate capacity reductions over a finite period of time and along a finite length of roadway with a given number of lanes affected. Therefore, in this study, estimated capacity loss is measured in vehicles.

In the course of this study, methods were developed for estimating the impacts of temporary events on the loss of capacity and delay, but not for estimating the impacts of temporary capacity losses on rescheduling, re-routing, reduced mobility, and reliability (the four Rs). Thus, the impacts of events on 
traffic volumes were not predicted. For example, a heavy snowstorm might reduce traffic volumes drastically due to travelers re-scheduling or canceling planned travel. On the other hand, because normal traffic volumes are assumed, delay will be overestimated. Thus, in general, the delay estimates presented here reflect, to an unknown degree, the other negative impacts of temporary capacity losses on the four Rs. A high priority for future analysis should be to develop methods for analyzing all five types of impacts.

\section{ES-4. RESULTS}

\section{Total Effects}

Temporary capacity losses due to work zones, crashes, breakdowns, adverse weather, sub-optimal signal timing, toll facilities, and railroad crossings caused over three and a half billion estimated vehicle-hours of delay on U.S. freeways and principal arterials in 1999 (Table ES-1). Assuming an average vehicle occupancy of 1.6 persons, this translates into nearly six billion person-hours of delay. Assuming an average value of time of $\$ 15$ per hour for each person impacted, temporary capacity losses produced approximately $\$ 55$ billion in lost time alone in 1999. Because conservative assumptions have been used throughout this analysis, and because several significant sources of delay have not been included, these estimates are believed to be a lower bound on the actual impacts of TLC.

Crashes and breakdowns account for over half the delay attributed to TLC events, and work zones account for about one quarter of the delay (Fig. ES-1). Over 85 percent of the delay from TLC events occurs in the off peak or on uncongested segments in the peak period of weekday recurring congestion (Table ES-2). Americans lose 2.5 hours for every 1,000 miles of travel due to delay from TLC events: delay is over 4 hours per 1,000 miles of travel in very large urban areas, about 3 hours and 45 minutes in large urban areas, over 2 hours in small and medium areas, and 45 minutes in rural areas (Table ES-3).

The TLC2 estimates were compared to two sets of delay estimates for 1999 by Texas Transportation Institute (TTI), including estimates for 85 urban areas in the 2004 Urban Mobility Study (Schrank and Lomax 2004) and unpublished estimates for all urban areas for FHWA. The TLC2 study estimates of total crash and breakdown delay are slightly higher than the TTI estimates for incident delay (Fig. ES-2). 
Table ES-1. Summary of capacity loss \& delay estimates for freeways \& principal arterials, 1999

\begin{tabular}{|c|c|c|c|c|}
\hline Event & $\begin{array}{l}\text { Total capacity loss* } \\
\text { (million vehicles) }\end{array}$ & $\begin{array}{c}\text { Total delay* } \\
\text { (million veh-hours) }\end{array}$ & $\begin{array}{l}\text { Average } \\
\text { delay/driver } \\
\text { (hours) }\end{array}$ & $\begin{array}{l}\text { Average } \\
\text { delay/event* } \\
\text { (veh-hours) }\end{array}$ \\
\hline Crashes & 3,290 & 1,680 & 9.0 & 506 \\
\hline Fatal & 30.5 & 13.7 & 0.1 & 754 \\
\hline Non-fatal & 3,250 & 1,660 & 8.9 & 505 \\
\hline Breakdowns & 7,480 & 440 & 2.4 & 15.9 \\
\hline Work zones & 8,350 & 889 & 4.8 & 836,000 \\
\hline Adverse weather & 20,900 & 330 & 1.8 & \\
\hline Fog & 410 & 5.79 & 0.03 & \\
\hline Rain & 16,200 & 236 & 1.3 & \\
\hline Snow & 3,290 & 43.8 & 0.2 & \\
\hline Ice & 929 & 44.8 & 0.2 & \\
\hline PUD activities & 117 & 0.950 & 0.01 & \\
\hline Railroad crossings & $\mathrm{NC}^{*}$ & 2.95 & 0.02 & \\
\hline Toll facilities & $\mathrm{NC}^{\ddagger}$ & 21.0 & 0.1 & \\
\hline Signal timing & 173,000 & 296 & 1.6 & 2,770 \\
\hline Total & & 3,660 & 19.5 & \\
\hline $\begin{array}{l}\text { Non-recurring } \\
\text { delay }\end{array}$ & & 3,340 & 17.9 & \\
\hline \multicolumn{5}{|c|}{$\begin{array}{l}\text { Due to significant uncertainty as to the accuracy of the estimates, all values in these columns are rounded to three } \\
\text { significant digits. Estimates in detailed tables in chapters 3-10 are not rounded; however, the number of decimal } \\
\text { places shown should not be considered an indication of the accuracy of those estimates. }\end{array}$} \\
\hline $\begin{array}{l}\text { Delay/driver is avera } \\
\text { crash. }\end{array}$ & across all licensed drive & in the U.S. rather than $\mathrm{f}$ & Irivers actually $\mathrm{d}$ & ayed by each \\
\hline
\end{tabular}


Table ES-2. Summaries of delay by area type \& size, highway type, and traffic period \& congestion level.

\begin{tabular}{|c|c|c|c|c|c|c|c|c|c|c|c|}
\hline & \multirow{2}{*}{$\begin{array}{c}\text { Share } \\
\text { of } \\
\text { total }\end{array}$} & \multicolumn{10}{|c|}{ Delay in million vehicle hours } \\
\hline & & Total & $\begin{array}{c}\text { Fatal } \\
\text { crashes }\end{array}$ & $\begin{array}{c}\text { Non-fatal } \\
\text { crashes }\end{array}$ & $\begin{array}{l}\text { Break- } \\
\text { downs }\end{array}$ & $\begin{array}{l}\text { Work } \\
\text { zones } \\
\end{array}$ & Weather & $\begin{array}{c}\text { Signal } \\
\text { timings } \\
\end{array}$ & $\begin{array}{l}\text { Railroad } \\
\text { crossings } \\
\end{array}$ & $\begin{array}{c}\text { Urban } \\
\text { PUD }\end{array}$ & $\begin{array}{c}\text { Toll } \\
\text { facilities } \\
\end{array}$ \\
\hline Total & $100 \%$ & $3,657.9$ & 13.7 & $1,664.2$ & 440.0 & 889.0 & 330.1 & 295.8 & 2.9 & 0.95 & 21.0 \\
\hline \multicolumn{12}{|l|}{ By area type $\&$ size* } \\
\hline Urban - Very large & $38 \%$ & $1,372.6$ & 2.5 & 808.8 & 155.3 & 169.3 & 122.2 & 112.9 & 0.7 & 0.9 & -- \\
\hline Urban - Large & $28 \%$ & $1,041.0$ & 8.4 & 520.6 & 91.6 & 282.6 & 80.5 & 56.8 & 0.4 & 0.09 & -- \\
\hline Urban - Medium & $8 \%$ & 295.2 & 0.1 & 106.2 & 24.5 & 106.3 & 29.6 & 28.2 & 0.2 & 0.001 & -- \\
\hline Urban - Small & $15 \%$ & 547.1 & 2.1 & 128.0 & 72.9 & 181.8 & 71.3 & 89.7 & 1.4 & 0.01 & -- \\
\hline Rural & $10 \%$ & 380.9 & 0.6 & 100.5 & 95.7 & 149.0 & 26.5 & 8.2 & 0.3 & -- & -- \\
\hline \multicolumn{12}{|l|}{ By highway type } \\
\hline Urban freeways \& expressways & $56 \%$ & $2,036.4$ & 6.1 & $1,196.1$ & 12.1 & 730.2 & 91.8 & -- & -- & -- & -- \\
\hline Urban other principal arterials & $33 \%$ & $1,219.5$ & 7.0 & 367.5 & 332.2 & 9.7 & 211.8 & 287.6 & 2.7 & 1.0 & -- \\
\hline Rural Freeways & $5 \%$ & 165.5 & 0.2 & 16.2 & 0.4 & 136.5 & 12.2 & -- & -- & -- & -- \\
\hline Rural other principal arterials & $6 \%$ & 215.4 & 0.4 & 84.3 & 95.3 & 12.5 & 14.3 & 8.2 & 0.3 & -- & -- \\
\hline \multicolumn{12}{|l|}{ By period \& congestion level $^{\dagger}$} \\
\hline Peak - Congested & $13 \%$ & 462.8 & 0.1 & 201.9 & 30.2 & 98.9 & 80.1 & 51.3 & 0.1 & 0.1 & -- \\
\hline Peak - Not congested & $27 \%$ & 992.5 & 3.6 & 495.4 & 133.5 & 243.6 & 50.6 & 64.8 & 0.8 & 0.2 & -- \\
\hline Off-peak & $60 \%$ & $2,181.5$ & 10.0 & 966.9 & 276.3 & 546.5 & 199.5 & 179.7 & 2.0 & 0.6 & -- \\
\hline
\end{tabular}

* Urban area size categories are based on population: very large - more than 3 million; large - 1 to 3 million; medium 0.5 to 1 million; small - less than 0.5 million.

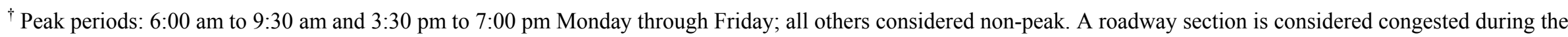
peak periods if its Volume/Service Flow Ratio (V/SF) is greater than $95 \%$. 
Table ES-3. Summaries of delay per thousand miles of travel.

\begin{tabular}{|c|c|c|c|c|c|c|c|c|c|c|c|}
\hline & \multirow[b]{2}{*}{$\begin{array}{c}\text { VMT } \\
\text { (millions) }\end{array}$} & \multicolumn{10}{|c|}{ Delay in hours per thousand miles of travel } \\
\hline & & Total & $\begin{array}{c}\text { Fatal } \\
\text { crashes }\end{array}$ & $\begin{array}{c}\text { Non-fatal } \\
\text { crashes }\end{array}$ & $\begin{array}{l}\text { Break- } \\
\text { downs }\end{array}$ & $\begin{array}{l}\text { Work } \\
\text { zones } \\
\end{array}$ & Weather & $\begin{array}{c}\text { Signal } \\
\text { timings } \\
\end{array}$ & $\begin{array}{l}\text { Railroad } \\
\text { crossings } \\
\end{array}$ & $\begin{array}{l}\text { Urban } \\
\text { PUD } \\
\end{array}$ & $\begin{array}{c}\text { Toll } \\
\text { facilities } \\
\end{array}$ \\
\hline Total & $1,451,424$ & 2.520 & 0.009 & 1.147 & 0.303 & 0.613 & 0.227 & 0.204 & 0.002 & 0.001 & 0.014 \\
\hline \multicolumn{12}{|l|}{ By area type \& size* } \\
\hline Urban - Very large & 329,032 & 4.172 & 0.008 & 2.458 & 0.472 & 0.515 & 0.371 & 0.343 & 0.002 & 0.003 & - \\
\hline Urban - Large & 277,885 & 3.746 & 0.030 & 1.873 & 0.330 & 1.017 & 0.290 & 0.205 & 0.001 & 0.000 & - \\
\hline Urban - Medium & 105,428 & 2.800 & 0.001 & 1.007 & 0.232 & 1.009 & 0.281 & 0.267 & 0.002 & 0.000 & - \\
\hline Urban - Small & 234,925 & 2.329 & 0.009 & 0.545 & 0.310 & 0.774 & 0.303 & 0.382 & 0.006 & 0.000 & - \\
\hline Rural & 504,154 & 0.756 & 0.001 & 0.199 & 0.190 & 0.296 & 0.053 & 0.016 & 0.001 & - & - \\
\hline \multicolumn{12}{|l|}{ By highway type } \\
\hline $\begin{array}{l}\text { Urban freeways \& } \\
\text { expressways }\end{array}$ & 554,549 & 3.672 & 0.011 & 2.157 & 0.022 & 1.317 & 0.166 & - & - & - & - \\
\hline $\begin{array}{l}\text { Urban other principal } \\
\text { arterials }\end{array}$ & 392,721 & 3.105 & 0.018 & 0.936 & 0.846 & 0.025 & 0.539 & 0.732 & 0.007 & 0.002 & - \\
\hline Rural freeways & 260,204 & 0.636 & 0.001 & 0.062 & 0.002 & 0.525 & 0.047 & - & - & - & - \\
\hline $\begin{array}{l}\text { Rural other principal } \\
\text { arterials }\end{array}$ & 243,950 & 0.883 & 0.002 & 0.346 & 0.391 & 0.051 & 0.059 & 0.034 & 0.001 & - & - \\
\hline \multicolumn{12}{|c|}{ By period \& congestion level ${ }^{\dagger}$} \\
\hline $\begin{array}{l}\text { Peak period - } \\
\text { Congested }\end{array}$ & 71,176 & 6.502 & 0.002 & 2.837 & 0.424 & 1.390 & 1.125 & 0.721 & 0.002 & 0.002 & - \\
\hline $\begin{array}{l}\text { Peak period - Not } \\
\text { congested }\end{array}$ & 421,524 & 2.355 & 0.009 & 1.175 & 0.317 & 0.578 & 0.120 & 0.154 & 0.002 & 0.000 & - \\
\hline Off-peak & 958,724 & 2.275 & 0.010 & 1.008 & 0.288 & 0.570 & 0.208 & 0.187 & 0.002 & 0.001 & - \\
\hline
\end{tabular}

* Urban area size categories are based on population: very large - more than 3 million; large - 1 to 3 million; medium 0.5 to 1 million; small - less than 0.5 million.

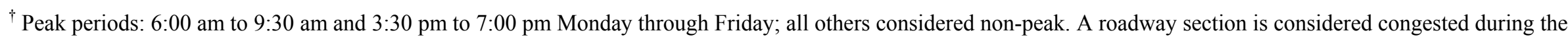
peak periods if its Volume/Service Flow Ratio (V/SF) is greater than 95\%. 


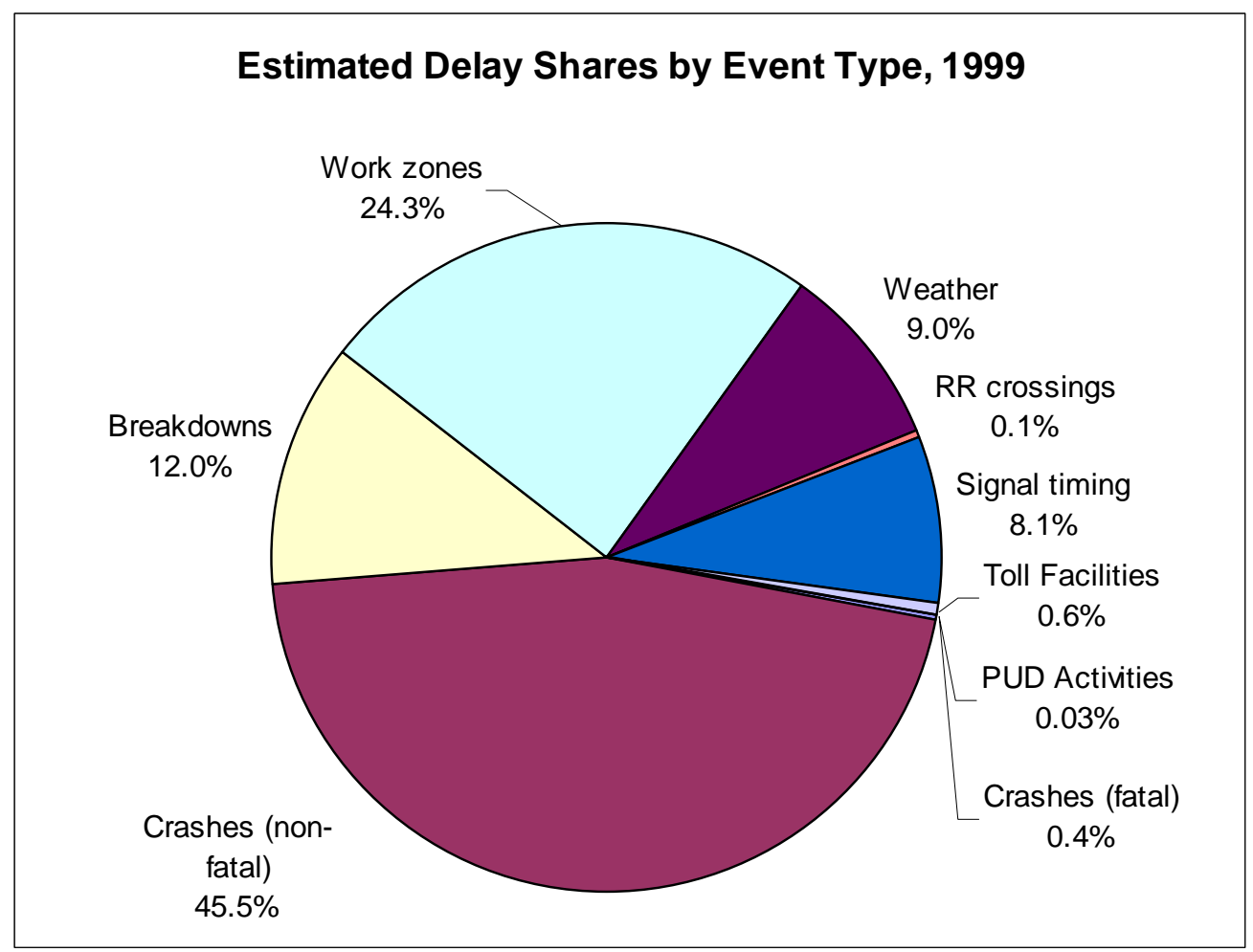

Fig. ES-1. Most of the delay attributed to TLC events was caused by crashes and work zone activities.

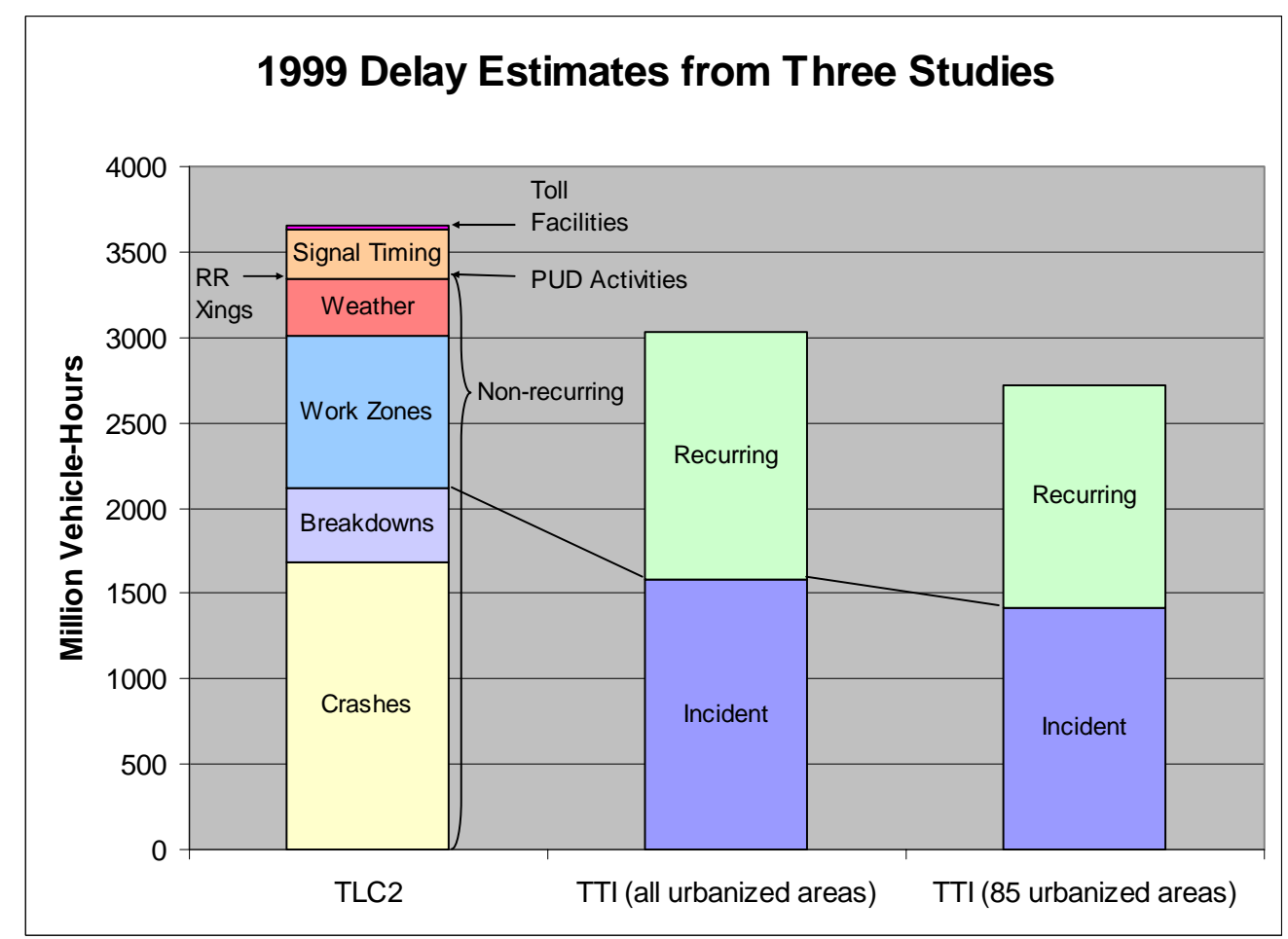

Fig. ES-2. A comparison of TLC2 estimates with those from two studies by TTI: one that includes 85 urbanized areas and another that includes all urbanized areas. 


\section{Crashes}

Crashes on freeways and principle arterials caused an estimated 1.7 billion vehicle-hours (approximately 2.7 billion person-hours) of delay in 1999. Estimated delays on freeways amounted to 1.2 billion vehiclehours, almost three times the delays from crashes on principal arterials. Non-fatal crashes were the primary source of delay due to their greater occurrence rate. The TLC2 study estimates that crashes were responsible for over 45 percent of delay caused by TLC events.

Several areas for improving crash impact estimates have been identified: (1) developing methods to estimate delays on transverse arterials (neglected here), (2) developing improved methods for geolocating non-fatal accidents, and (3) developing means of validating the Monte Carlo methods used in conjunction with the GES crash data.

\section{Breakdowns}

It is estimated that vehicle breakdowns caused approximately 440 million vehicle-hours $(0.7$ billion person-hours) of delay in 1999, accounting for 12 percent of delays from TLC events. Information about breakdowns is scarce, making the associated capacity loss and delay estimates one of the weakest of all impacts estimated. Better data are needed on virtually all aspects of breakdowns. In particular, information regarding the total number of breakdowns or vehicle breakdown rates would improve estimates greatly, as would case studies of the impacts of breakdowns under a variety of circumstances.

\section{Work Zones}

Work zones on freeways and principal arterials caused an estimated 889 million vehicle-hours (1.4 billion person-hours) of delay in 1999. The majority of delay (90 percent) is associated with the transition area of the work zone rather than the activity area. Work zones accounted for about a quarter of all estimated delay from TLC sources within the scope of TLC2.

Work zone impact estimates are believed to be low for two principal reasons. First, the estimates presented in this report are based on freeway and principal arterial construction only. Delay from construction and maintenance on local arterials was not estimated. Second, the research team believes the Rand McNally database under-represents construction projects scheduled for more than four months in the future.

\section{Weather}

Major events of fog, rain, snow, and ice combined to cause an estimated 330 million vehicle-hours $(0.5$ billion person-hours) of delay on freeways and principal arterials in 1999. These adverse weather events accounted for 9 percent of delay from TLC sources. Rain was estimated to be the most significant weather factor, accounting for 71 percent of the estimated weather delay.

The methods used to estimate capacity reductions and delays due to weather have several shortcomings. First, as for other events in the study, the impacts of weather events on traffic volumes were beyond the study's scope and were not considered. Thus, the impacts of major snowstorms and ice are estimated assuming normal traffic volumes. In reality, a substantial fraction of the impacts of such conditions is likely to be in re-scheduled or reduced travel, and these impacts were not estimated. As a result, the delay impacts, per se, have likely been overestimated, while the total impacts may be underestimated. Second, the duration of weather impacts is undoubtedly underestimated due to data limitations. Weather data includes the duration of weather events (such as precipitation falling as snow), but there is no data on how long roadways are actually affected by these events. For example, while the visibility impacts of fog last 
only as long as the event itself, a six-hour snowstorm may reduce capacity for several hours (or days in some areas) after it stops snowing. In order to be conservative in estimating delays, in the absence of impact duration data, the study assumes that the duration of capacity reduction coincides with the duration of the weather event rather than the duration of its impact. Third, capacity reductions and delays from several weather-related events are not included in the study. This includes capacity reductions caused by reduced visibility from solar glare, dust storms, and forest fires, as well as reduced capacity caused by strong winds on high-profile vehicles. Likewise, the impacts of catastrophic events such as hurricanes, floods, and other natural disasters were not considered. Finally, the quality and spatial resolution of the weather data limit the accuracy of capacity reduction and delay estimates.

\section{Sub-optimal Signal Timing}

Sub-optimal traffic controls caused an estimated 0.3 billion vehicle-hours ( 0.5 billion person-hours) of delay on principal arterials in 1999-about 8 percent of delay estimated by this study. These estimates differ from the other delay estimates in TLC2 in that they represent the potential for benefits due to improved operations. Delays caused by fixed-time and actuated signals appear to be roughly proportional to the number of signals of each type.

These impact estimates are believed to be among the most reliable, although a number of areas for refinement have been identified.

\section{Railroad Crossings}

It is estimated that railroad crossings caused about 3 million vehicle-hours ( 4.7 million person-hours) of delay on principal arterials in 1999-freeways do not have railroad crossings. At-grade railroad crossings caused only about 0.1 percent of delay in 1999.

\section{Toll Facilities}

Toll facilities caused an estimated 21 million vehicle-hours (about 34 million person-hours) of delay on freeways and principal arterials in 1999. Average delay per transaction was estimated at about 12 seconds. TLC2 estimates that toll facilities were responsible for only half a percent of the delay attributable to TLC events within the scope of the study, second only to at-grade railroad crossings.

\section{PUD Activities in Urban Areas}

Large truck pickup and delivery (PUD) activities can cause delay if the truck is parked such that it blocks a lane of traffic. The study estimates that lane-blocking PUD activities in urban areas resulted in just under a million vehicle-hours of delay in 1999. The PUD-related delay share was less than three hundredths of a percent, the smallest impact from any source in the study.

\section{ES-5. COMPOSITE PICTURE AND NEXT STEPS}

No single empirical or modeling study provides a comprehensive estimate of all sources of delay. However, an approximate picture of total delay can be assembled by combining TTI's estimate of recurring delay for all urban areas, the TLC2 estimate of recurring delay from suboptimal signal timing and tollbooths, and TLC2 estimates of nonrecurring delay. The resulting 5.1 billion hours of delay is 35 percent recurring and 65 percent nonrecurring. Recurring delay is a higher percentage in the larger cities. 
While the TLC2 study breaks new ground in estimating capacity loss and delay from a wide variety of sources, it is only the beginning of needed efforts to understand all forms of congestion and their consequences. No national studies examine recurring congestion from weekend and holiday travel or recurring weekday congestion in rural areas. The full effects of bottlenecks, the extent and intensity of most forms of temporary capacity reductions, and the consequences of dramatic increases in trucking are not adequately understood or based on robust empirical studies. Delay on roads smaller than freeways and other major arteries; the interactions among different types of delay; relationships between temporary capacity loss and mitigation strategies; and the effects of re-routing, rescheduling, reduced mobility, and reduced reliability are additional areas requiring data collection and analysis. 


\section{INTRODUCTION}

\subsection{BACKGROUND}

Traffic congestion and its impacts significantly affect the nation's economic performance and the public's quality of life. In most urban areas, travel demand routinely exceeds highway capacity during peak periods. In addition, events such as crashes, vehicle breakdowns, work zones, adverse weather, railroad crossings, commercial truck pickup and delivery (PUD) activities in urban areas, and other factors such as toll collection facilities and sub-optimal signal timing cause temporary capacity losses, often worsening the conditions on already congested highway networks. The impacts of these temporary capacity losses include delay, reduced mobility, and reduced reliability of the highway system. They can also cause drivers to re-route or re-schedule trips. Such information is vital to formulating sound public policies for the highway infrastructure and its operation.

In response to this need, Oak Ridge National Laboratory, sponsored by the Federal Highway Administration (FHWA), made an initial attempt to provide nationwide estimates of the capacity losses and delay caused by temporary capacity-reducing events (Chin et al. 2002). This study, called the Temporary Loss of Capacity (TLC) study, estimated capacity loss and delay on freeways and principal arterials resulting from fatal and non-fatal crashes, vehicle breakdowns, and adverse weather, including snow, ice, and fog. In addition, it estimated capacity loss and delay caused by sub-optimal signal timing at intersections on principal arterials. It also included rough estimates of capacity loss and delay on Interstates due to highway construction and maintenance work zones. Capacity loss and delay were estimated for calendar year 1999, except for work zone estimates, which were estimated for May 2001 to May 2002 due to data availability limitations. Prior to the first phase of this study, which was completed in May of 2002, no nationwide estimates of temporary losses of highway capacity by type of capacityreducing event had been made.

This report describes the second phase of the TLC study (TLC2). TLC2 improves upon the first study by expanding the scope to include delays from rain, toll collection facilities, railroad crossings, and commercial truck PUD activities in urban areas. It includes estimates of work zone capacity loss and delay for all freeways and principal arterials, rather than for Interstates only. It also includes improved estimates of delays from fog, snow, and ice, which are based on data not available during the initial phase of the study.

Finally, it should be noted that, in the process of expanding the scope and improving the data sources and methodologies for TLC2, it was discovered that a computer-processing error generated incorrect capacity reduction and delay estimates for crashes and breakdowns presented in the original study report (Chin et al. 2002). This error affects all crash and breakdown estimates in that report. The authors have corrected this error and re-calculated the crash and breakdown estimates. These revised estimates are included in the TLC2 report. The specific estimates in the initial TLC report that should be disregarded are given in Table 1 below. 
Table 1. Results in initial TLC report that should be disregarded due to a computer processing error

\begin{tabular}{l|l|c|l}
\hline Chapter & Table/figure no. & Page(s) & Erroneous estimates \\
\hline \multirow{2}{*}{$\begin{array}{l}\text { Executive } \\
\text { Summary }\end{array}$} & Table ES-1 & xii & Estimates for crashes, breakdowns, and all summary totals \\
\cline { 2 - 4 } & Figs. ES-1 \& ES-2 & xiii & Estimates for crashes, breakdowns, and all summary totals \\
\hline \multirow{2}{*}{ Ch. 3} & Tables $10-13$ & $20-24$ & All estimates \\
\cline { 2 - 4 } & Figs. 8 \& 9 & $20-21$ & All estimates \\
\hline Ch. 4 & Table 14 & 27 & All estimates \\
\hline Ch. 5 & Table 18 & 32 & All estimates for fatal crashes \\
\hline \multirow{2}{*}{ Ch. 8} & Table 28 & 53 & Estimates for crashes, breakdowns, and all summary totals \\
\cline { 2 - 4 } & Fig. 17 & 53 & All values \\
\cline { 2 - 4 } & Fig. 18 & 54 & Estimates for crashes, breakdowns, and totals \\
\hline
\end{tabular}

\subsection{OBJECTIVE AND SCOPE}

The objective of the TLC study was to develop and implement methods for producing national-level estimates of the loss of capacity on the nation's highway facilities due to temporary phenomena as well as estimates of the impacts of such losses. The objective of TLC2 was to improve these estimates by expanding the scope to include phenomena not included in the first study and to refine the initial estimates in cases where improved data and/or methodologies could be employed.

The scope of TLC2 includes all urban and rural freeways and principal arterials in the nation's highway system for 1999-delays on minor arterials, collectors, and local roads are not included. These roadways accounted for about 54 percent of the highway vehicle-miles of travel (VMT) in 1999 (Fig. 1). Specifically, this study attempts to quantify the extent of temporary capacity losses due to crashes, breakdowns, work zones, weather, sub-optimal signal timing, railroad crossings, toll facilities, and commercial truck PUD activities in urban areas. These events can cause impacts such as capacity reduction, delays, trip rescheduling, rerouting, reduced mobility, and reduced reliability. This study focuses on the reduction of capacity and resulting delays caused by the temporary events mentioned above. Impacts other than capacity losses and delay, such as re-routing, re-scheduling, reduced mobility, and reduced reliability, are not covered in this phase of research.

It should also be noted that the study does not attempt to estimate capacity losses and delays due to events that occur simultaneously, such as a crash that takes place during a snowstorm or a breakdown that takes place in a work zone. Such coinciding events can often cause more capacity loss and delay than they might have caused if they had occurred separately. The interaction of capacity-reducing events is an area of interest and may be addressed in future research. However, due to time and funding constraints of the initial phases of the study, this interaction was not modeled. 


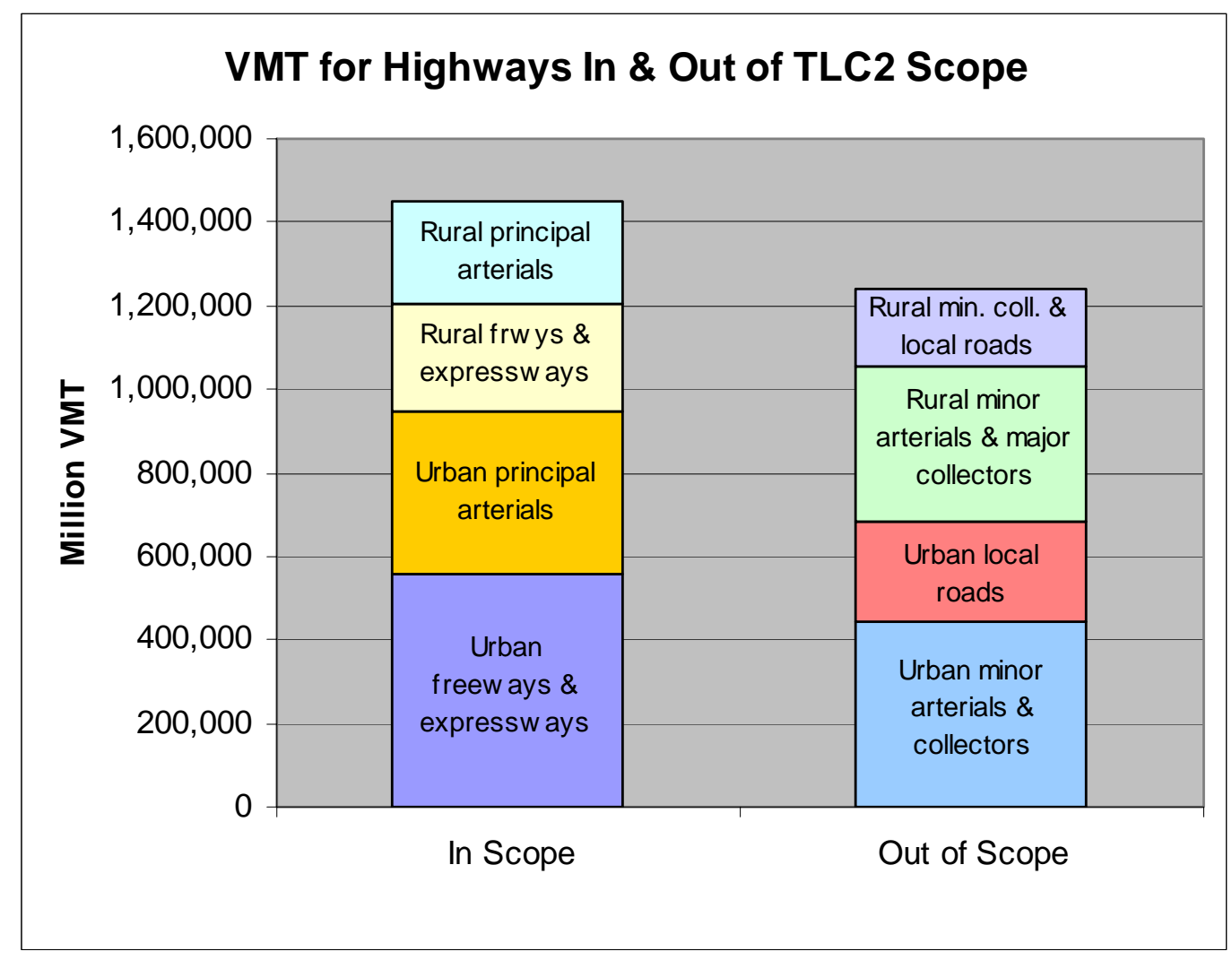

Fig. 1. The highways within the scope of TLC2 accounted for about 54 percent of the VMT in the U.S. in 1999.

\subsection{IMPORTANT TERMS USED IN THIS REPORT}

Definitions of urban areas, peak periods, and traffic conditions are made as consistent as possible with both FHWA practice and congestion measures published by the Texas Transportation Institute (TTI).

Urban areas are identified in Table HM-72 of FHWA's Highway Statistics 1999 and are classified into the size categories in Table 2. These FHWA-recognized urban areas are urbanized areas defined by the U.S. Bureau of the Census with populations of at least 50,000 - boundaries may be adjusted from the Census definition by local transportation officials. TTI used 68 of these areas, including the largest urban areas and a sponsor-selected set of smaller areas, in its 2001 Urban Mobility Report (Schrank and Lomax, 2001). TTI used all FHWA-recognized urban areas in unpublished tabulations for FHWA cited in chapter 11 of this report.

Table 2. Population ranges for urban area size categories

\begin{tabular}{l|c}
\hline Urban area size & Population \\
\hline Very large & $>3,000,000$ \\
\hline Large & $1,000,000$ to $3,000,000$ \\
\hline Medium & 500,000 to $1,000,000$ \\
\hline Small & $<500,000$ \\
\hline
\end{tabular}


Peak periods are assumed to be from 6:00 am to 9:30 am in the morning and 3:30 pm to 7:00 pm in the afternoon from Monday to Friday, to be consistent with TTI's annual mobility report. All other times of the day and days of the week are considered off-peak periods.

Traffic conditions for each roadway segment during peak periods are determined by the Volume/Service Flow Ratio (V/SF) information provided in the sample section of the Highway Performance Monitoring System (HPMS) data. A roadway section is considered congested during the peak periods if its V/SF is greater than $95 \%$. This V/SF is a computed value reflecting peak hour congestion for a sample section. It is used in investment requirements modeling to estimate needed capacity improvements, in the national highway database, and for congestion, delay, and other data analyses. This value is generated by the HPMS software from HPMS data and procedures are described in Appendix N, Highway Performance Monitoring System Field Manual (U.S. DOT/FHWA 2000).

\subsection{ORGANIZATION OF THE REPORT}

This report begins by describing the general approach and methodology used in TLC and TLC2 to estimate capacity reduction and delay. It explains the basic concepts of capacity, capacity reduction, and delay and provides a number of derivations, data sources, and assumptions used throughout the study.

Chapters 3 through 10 describe the specific methodology, data, and assumptions used to estimate capacity loss and delay for each event type. These include

- Crashes (both fatal and non-fatal)

- Breakdowns

- Work zones

- Weather events (rain, fog, ice, and snow)

- Sub-optimal signal timing (principal arterials only)

- Highway-railroad crossings (principal arterials only)

- Toll facilities

- Commercial truck pickup and delivery (PUD) activities (urban principal arterials only)

These chapters also present the estimated capacity loss and delay for each event type and provide information on the reliability of the estimates. Capacity reduction and delay statistics are organized by area type (rural, very large urban, large urban, medium urban, and small urban) and time period category (peak and off-peak), where possible. Peak period estimates are further divided into those occurring under congested conditions and those occurring during under un-congested conditions.

Chapter 11 briefly summarizes the estimates for all event types in the TLC 2 study and compares them with delay estimates generated by the Texas Transportation Institute (TTI) in their 1999 Urban Mobility Study (Schrank and Lomax 2001), as well as an expanded study performed for FHWA.

The report concludes by identifying areas for further research in chapter 12 . 


\section{APPROACH AND GENERAL METHODOLOGY}

\subsection{APPROACH}

The study uses traffic engineering modeling methods, the best available data, and engineering judgment to derive estimates of capacity losses and delays. Because direct measurements are scarce and available data are generally incomplete, the validity of the estimates is dependent on the reasonableness of a number of critical assumptions. The study used rigorous procedures and methodologies firmly grounded in traffic theory and practice, but attempted to keep the methods as straightforward and transparent as possible. Existing data from a variety of sources was used, as well as results from evaluation studies sponsored by the Intelligent Transportation System (ITS) Joint Program Office (JPO). Reliable data was used where possible. For areas where data was not available, data or findings from literature sources, reasonable assumptions, and Monte Carlo simulations were used. The philosophy followed throughout the study was to rely on published peer-reviewed studies whenever possible and, when assumptions must be based solely on the researchers' judgment, to err on the side of underestimating losses of capacity and delay. There is one general exception to this rule: the assumption that traffic volumes are not changed by the occurrence of a TLC event. This issue is discussed below.

The study's analytical framework is portrayed in Fig.2. Events causing temporary capacity losses occur in an environment comprised of roadway characteristics, location, time, and ambient conditions (e.g., weather). The characteristics of the event and its environment provide the information based on which traffic impact models can predict impacts. These travel impacts include delay and the four Rs: (1) rerouting, (2) re-scheduling, (3) reduced mobility (foregone travel, cancelled trips), and (4) reduced reliability. In general, delay is probably the more useful, and certainly the more intuitive, measure of loss of functionality. In general, an event will generate all five types of impacts, with the relative importance of each depending on the nature of the event and its context. For example, an unexpected event such as a crash is likely to produce relatively less re-routing and re-scheduling than a work zone whose existence can be known in advance and which may persist for days, weeks, or even longer.

A critical distinction is made between the loss of capacity and its impacts. Capacity is a measure of potential: it describes the maximum sustainable throughput of a highway. As such, it is independent of the highway's actual use. Impacts, however, depend not only on the loss of capacity, but also on the volume of traffic on the highway when the loss occurs. A crash occurring on an Interstate highway in the middle of the night will cause far less delay than, but possibly the same loss of capacity as, the same crash occurring during rush hour. Delay is measured in vehicle-hours, which can be converted to person-hours by multiplying by an appropriate vehicle occupancy. Capacity loss, on the other hand, is a loss of potential throughput (measured in vehicles per lane per hour, or vplph), integrated over time and a length of roadway.

Another distinction is important to note. While capacity is typically given as a rate of vehicle throughput, the capacity reduction estimates presented in this study are not given as a rate. Since the general methodology used in this study attempts to estimate capacity reductions over a finite period of time and along a finite length of roadway with a given number of lanes affected, the algorithm produces an estimate of vehicles not serviced through those lanes during the given amount of time. Thus, capacity loss is given in units of vehicles rather than vehicles per hour per lane. The more typical unit of capacity, in terms of vphpl, was used in the algorithms to estimate total capacity loss and delay.

In the course of this study, methods were developed for estimating the impacts of temporary events on the loss of capacity and delay, but not for estimating the impacts of TLCs on the four Rs. Thus, the impacts of 
events on traffic volumes were not predicted. For example, a heavy snowstorm might reduce traffic volumes drastically due to travelers re-scheduling or canceling planned travel. Such impacts have not been estimated. On the other hand, because normal traffic volumes are assumed, delay will be overestimated. Thus, in general, the delay estimates presented here reflect, to an unknown degree, the other negative impacts of TLCs on re-scheduling, re-routing, reduced mobility, and reliability. A high priority for future analysis should be to develop methods for analyzing all five types of impacts.

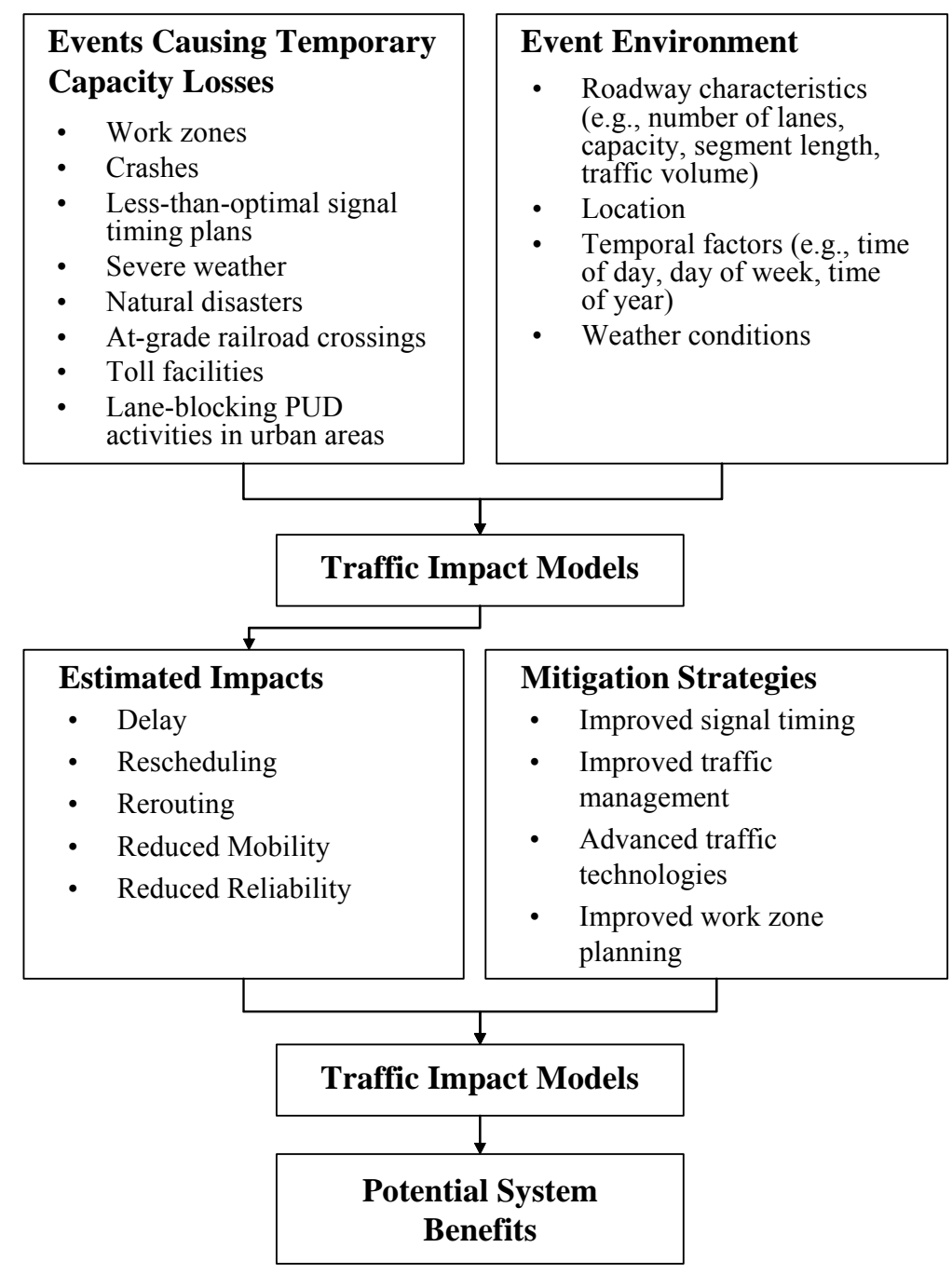

Fig. 2. Analytical Framework

\subsection{BASIC CONCEPTS OF CAPACITY, CAPACITY REDUCTION, AND DELAY}

This section describes the general concepts and methods used in this study to calculate the impacts of TLC events on freeways and major highways. Methodologies to measure other adverse consequences, such as trip re-scheduling, trip re-routing, reduced mobility (i.e., trip cancellation), and to describe the impacts on reliability should be considered candidates for further research. 


\subsubsection{Determining Roadway Capacity}

Roadway capacity is the maximum vehicular flow rate at a point on a segment of the roadway. The capacity of a roadway segment with relatively homogeneous physical and operational characteristics is the maximum number of vehicles the segment can accommodate within a unit of time. In cases where the capacity is different at different points on the segment, the point with the least capacity is considered to represent the capacity for the entire segment. For example, for a roadway segment with a narrow bridge, the capacity of the narrow bridge is assumed to represent the capacity of the roadway segment. Usually, an access-controlled, divided, multi-lane highway can carry approximately 2,000 vehicles per hour per lane (vphpl). This results in a total of approximately 48-thousand vehicles per day per lane or over 17.5 million vehicles per year per lane.

\subsubsection{Estimating Capacity Reductions}

Traffic flow theory asserts that, in a steady state, the flow-density curve of a roadway has a shape similar to the one shown in Fig. 3. For a given demand Q1, the segment of freeway that is represented by the density-flow curve operates at a density D1 and at a speed S1, represented by the slope of the line from the origin to point P1. The segment can also operate at higher densities and slower speeds (point P2), but with unstable flow. The maximum of the flow-density curve is the segment's capacity $\mathrm{C}$.

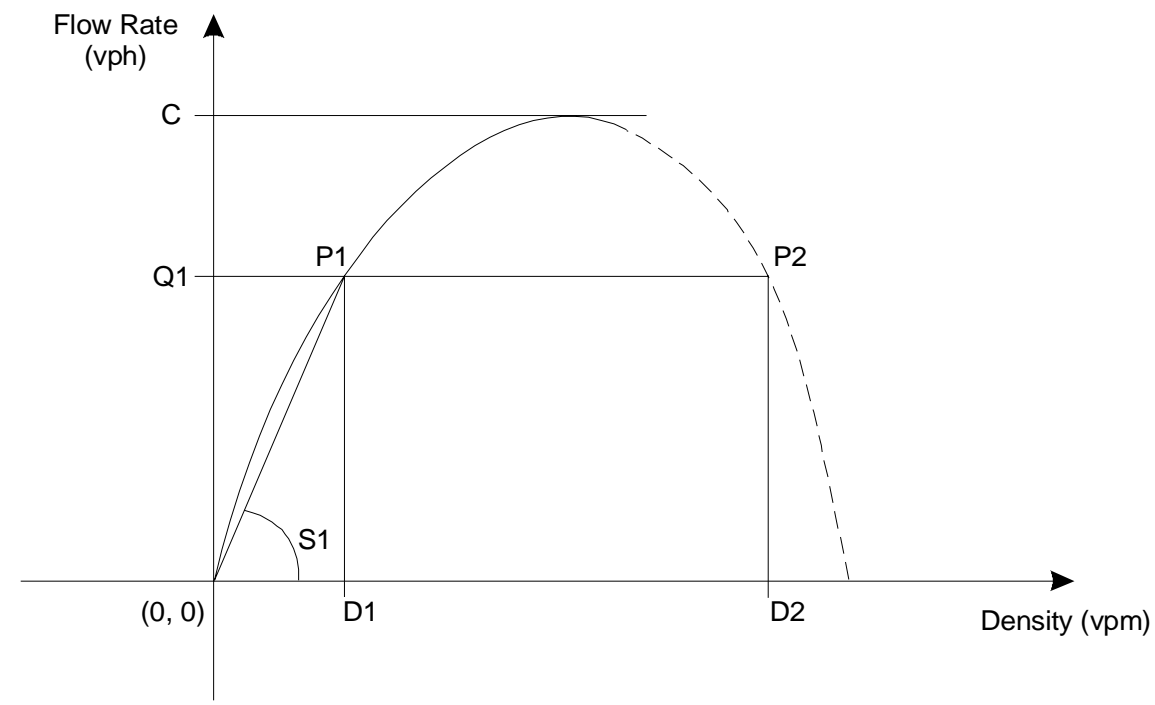

Fig. 3. Flow rate vs. density for a highway segment with capacity $C$ and a highway segment with capacity $C^{\prime}=\alpha C$

Assuming a segment of freeway, with a given capacity $\mathrm{C}$, and a section within that segment with a reduced capacity $C^{\prime}=\alpha C$, where $\alpha<1$, capacity $C^{\prime}$ was computed using the capacity reduction factor $\alpha$ for incidents obtained from Blumentritt et al. (1981) (Table 3). For work zones, the entire term $\mathrm{C}^{\prime}$ was obtained from the Highway Capacity Manual, Special Report 209 (TRB 1985) (Table 4). 
Table 3. Capacity reduction due to freeway incidents

\begin{tabular}{lrrrc}
\hline & \multicolumn{4}{c}{ Highway with } \\
\hline Incident type & 2 lanes & 3 lanes & 4 lanes & 5 lanes \\
\hline Vehicle moved to shoulder & $25 \%$ & $16 \%$ & $11 \%$ & -- \\
1 lane blocked & $68 \%$ & $47 \%$ & $44 \%$ & $25 \%$ \\
2 lanes blocked & $100 \%$ & $78 \%$ & $66 \%$ & $50 \%$ \\
\hline
\end{tabular}

Confidence: $+/-5 \%$ for small numbers; $+/-10 \%$ for large numbers

Table 4 shows the freeway capacity at work zones as a function of the total number of lanes and the number of lanes open. More recently, Dixon et al. (1996) analyzed speed-flow behavior in work zones in North Carolina. The study also presents a comparison with an earlier research conducted in Texas (see Table 5). The differences in the results from those two states are less than 10 percent.

Table 4. Average measured work zone capacities

\begin{tabular}{ccc}
\multicolumn{2}{c}{ Number of lanes } \\
Normal & Open & $\begin{array}{c}\text { Average capacity } \\
\text { (vphpl) }\end{array}$ \\
\hline 3 & 1 & 1,170 \\
2 & 1 & 1,340 \\
5 & 2 & 1,370 \\
4 & 2 & 1,480 \\
3 & 2 & 1,490 \\
4 & 3 & 1,520 \\
\hline
\end{tabular}

Caveat: Data has high variation from site to site.

Table 5. North Carolina and Texas work zone capacities

\begin{tabular}{|c|c|c|c|c|c|}
\hline \multicolumn{2}{|c|}{ No. of lanes } & \multirow[b]{2}{*}{$\begin{array}{l}\text { Rural or } \\
\text { urban }\end{array}$} & \multicolumn{2}{|c|}{ North Carolina* } & \multirow{2}{*}{$\begin{array}{c}\text { Texas** } \\
\begin{array}{l}\text { End of transition } \\
\text { (vphpl) }\end{array}\end{array}$} \\
\hline Normal & Open & & $\begin{array}{c}\text { End of transition } \\
\text { (vphpl) }\end{array}$ & $\begin{array}{l}\text { Activity area } \\
\text { (vphpl) }\end{array}$ & \\
\hline 2 & 1 & Rural & 1,300 & 1,210 & --- \\
\hline 2 & 1 & Urban & 1,690 & $1,515 * * *$ & 1,575 \\
\hline 3 & 1 & Urban & 1,640 & 1,440 & 1,460 \\
\hline
\end{tabular}

* Capacities

** Queue discharge

$* * *$ Two values reported: 1,560 and 1,490 for moderate and heavy work activity, respectively

Fig. 4 shows the flow-density curves for the freeway segment with capacity $\mathrm{C}$ (curve fd) and for the section with reduced capacity $\mathrm{C}^{\prime}$ (curve $f d^{\prime}$ ). 


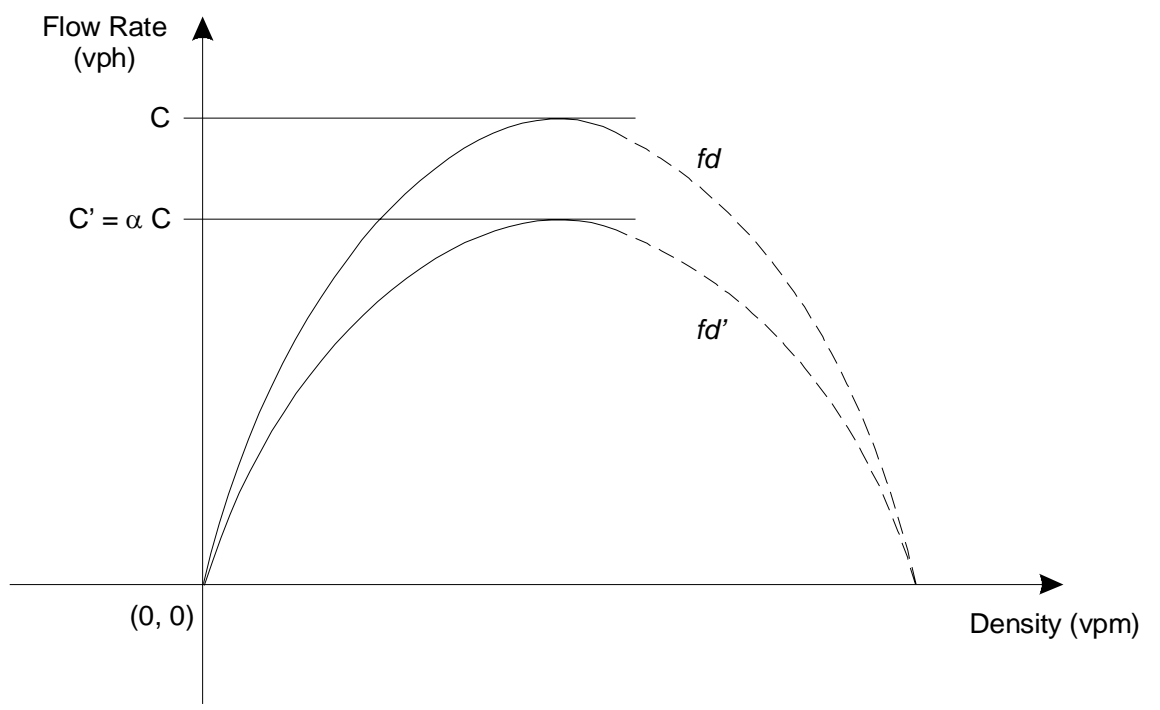

Fig. 4. Flow rate vs. density for a highway segment

Two cases must be analyzed: one in which the demand on the highway segment is less or equal to the reduced capacity $\mathrm{C}^{\prime}$, and one in which the demand is larger than $\mathrm{C}^{\prime}$.

\section{Case 1: Demand Less Than or Equal to the Reduced Capacity}

The first case examined is where the demand is equal to or less than the reduced capacity (Fig. 5). Upstream of the bottleneck (i.e., the freeway section with capacity $\mathrm{C}^{\prime}$ ), the highway is operating at a density D1 for the current demand Q1, with an average speed of S1 $\mathrm{mph}$ as described above (curve fd). Within the reduced capacity section, the flow-density curve is $f d^{\prime}$ and, at demand Q1, that section is operating at a density D2 ( $>$ D1) with average speeds of S2 mph $(<\mathrm{S} 1)$. Downstream of the bottleneck, the highway has capacity C, the density (with demand Q1) decreases again to D1, and the average speed increases from S2 to S1. Thus, demand Q1 is served within the three sections (i.e., upstream of bottleneck, bottleneck, and downstream of bottleneck), with average delays resulting from the difference between speeds S1 and S2.

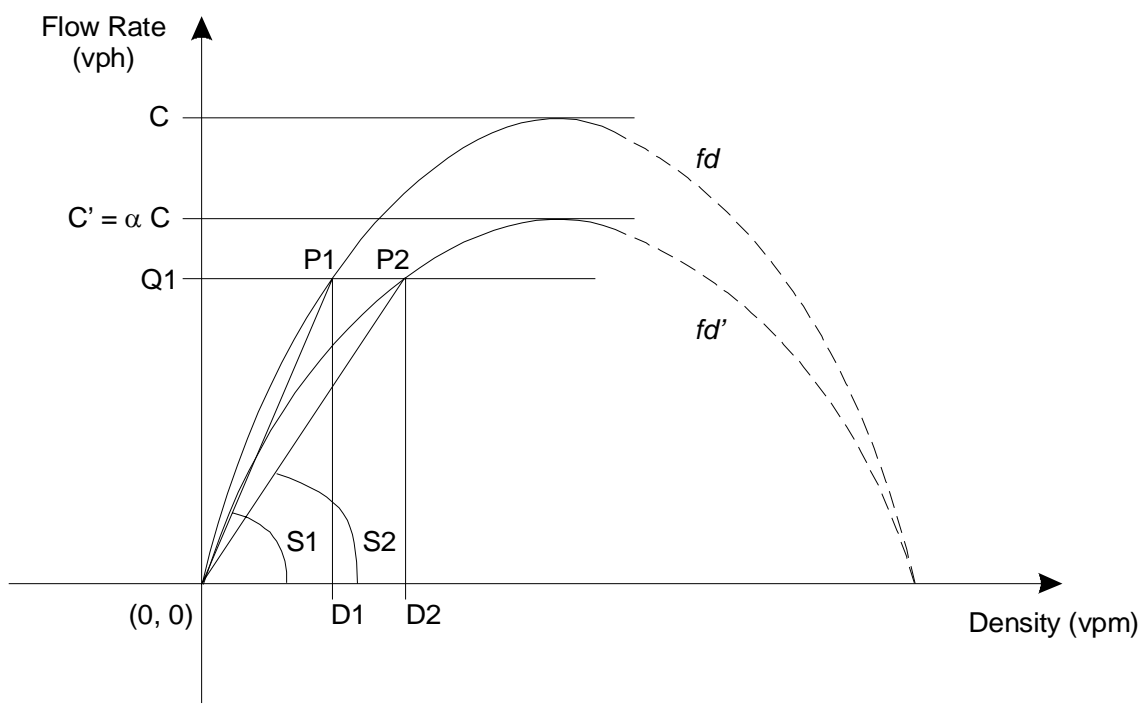

Fig. 5. Demand $Q 1$ equal to or less than bottleneck capacity $C^{\prime}=\alpha C$ 
Assuming the bottleneck section is L' miles long, the average delay can be computed as follows. The travel time (TT) in seconds for the bottleneck section under normal conditions (i.e., no incident) is

$$
T T=\frac{L^{\prime}}{S 1} \times 3,600
$$

where $\mathrm{S} 1$ is the speed in mph obtained from curve $f d$ for demand Q1, and 3,600 is merely a factor for converting hours to seconds. Under reduced capacity conditions, the travel time (in seconds) within the bottleneck section (TT') becomes

$$
T T^{\prime}=\frac{L^{\prime}}{S 2} \times 3,600
$$

where $\mathrm{S} 2$ is the speed in mph obtained from curve $f d^{\prime}$ for demand Q1. The average delay each vehicle experiences $\left(\mathrm{AD}_{1}\right)$ is calculated as the difference between these average travel times:

$$
A D_{1}=T T^{\prime}-T T=\frac{S 1-S 2}{S 1 \times S 2} \times L^{\prime} \times 3,600
$$

where $\mathrm{AD}_{1}$ is given in seconds per vehicle.

This statistic may be consequential for work zones where L' could be significant. For incidents, it may be irrelevant (i.e., $L^{\prime}$ is almost 0 ), but it could be used as a proxy for the rubbernecking delays at the incident location. To account for this effect, this study assumes a length of $L^{\prime}=1 / 2$ mile for incidents and computes the travel time delays on this segment.

\section{Case 2: Demand Greater than the Reduced Capacity}

The next case examined is where demand Q1 is larger than the capacity $\mathrm{C}^{\prime}$ of the bottleneck section (Fig. 6). If there were no loss of capacity, the highway would operate at point P1 with a density D1 and an average speed of S1 mph. However, because of the bottleneck, only a demand equal to $\mathrm{C}^{\prime}$ can be passed through the reduced capacity section, which will operate at density D2 and speed S2 (point P2). Downstream of the bottleneck, the freeway has a normal capacity $\mathrm{C}$, and, with a demand $\mathrm{C}^{\prime}$ (i.e., the demand that the bottleneck section can handle), it operates at point P3 with density D3 and speed S3 > S1.

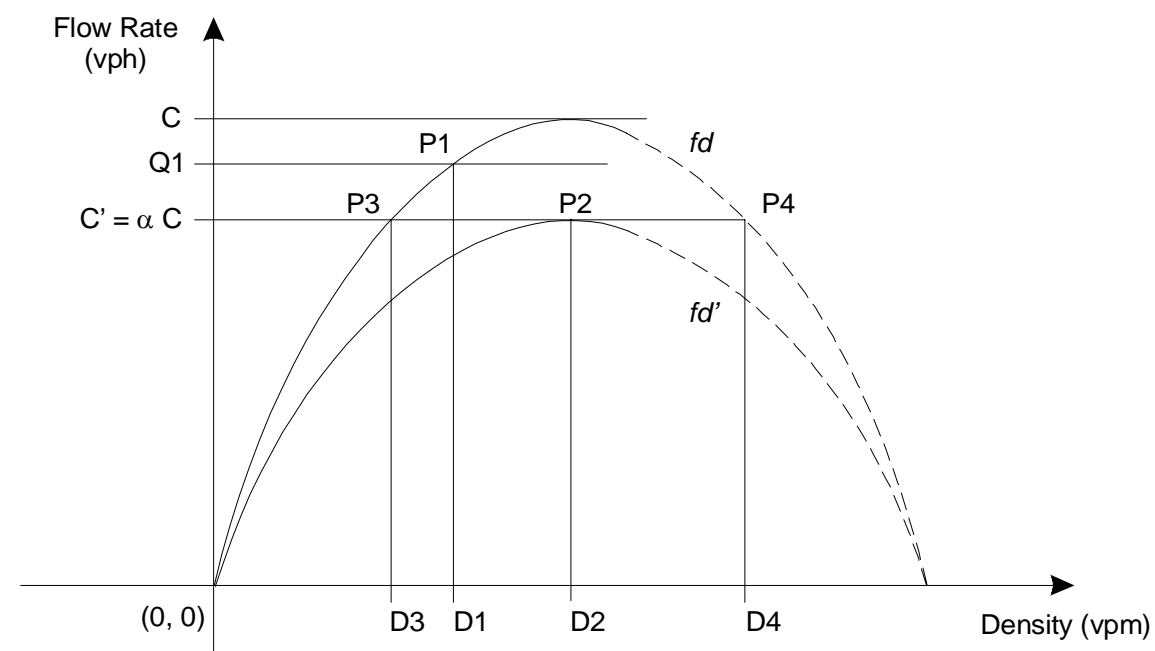

Fig. 6. Demand $Q 1$ larger than bottleneck capacity $C^{\prime}=\alpha C$ 
Upstream of the bottleneck, the freeway also has capacity $\mathrm{C}$ and operates as described by flow-density curve $f d$. However, because only demand $C^{\prime}$ can pass through the bottleneck section, vehicles begin to accumulate upstream of the bottleneck, increasing the density. Thus, this section of the freeway operates at point P4 along line $f d$ with a density D4 which is greater than D1 (the density of the areas upstream of the bottleneck not yet affected by it) and greater than D2 (the density at which the bottleneck section is operating).

Due to the difference between $\mathrm{Q} 1$ and $\mathrm{C}^{\prime}$, a queue builds up upstream of the bottleneck section at a rate indicated by Q1 - C'. Calling SW the speed at which this queue grows (also known as the "shock wave" speed), it is possible to write the following equation:

$$
Q 1-C^{\prime}=S W \times(D 4-D 1)
$$

where

$$
S W=\frac{Q 1-C^{\prime}}{D 4-D 1}
$$

\subsubsection{Estimating Delays for Localized Events}

Fig. 7 shows the effect over time that a loss of capacity can have on a section of freeway. This can also be used to compute several statistics, such as total duration of the congestion produced by the incident, total number of cars affected, the maximum individual delay, and the average delay per vehicle.

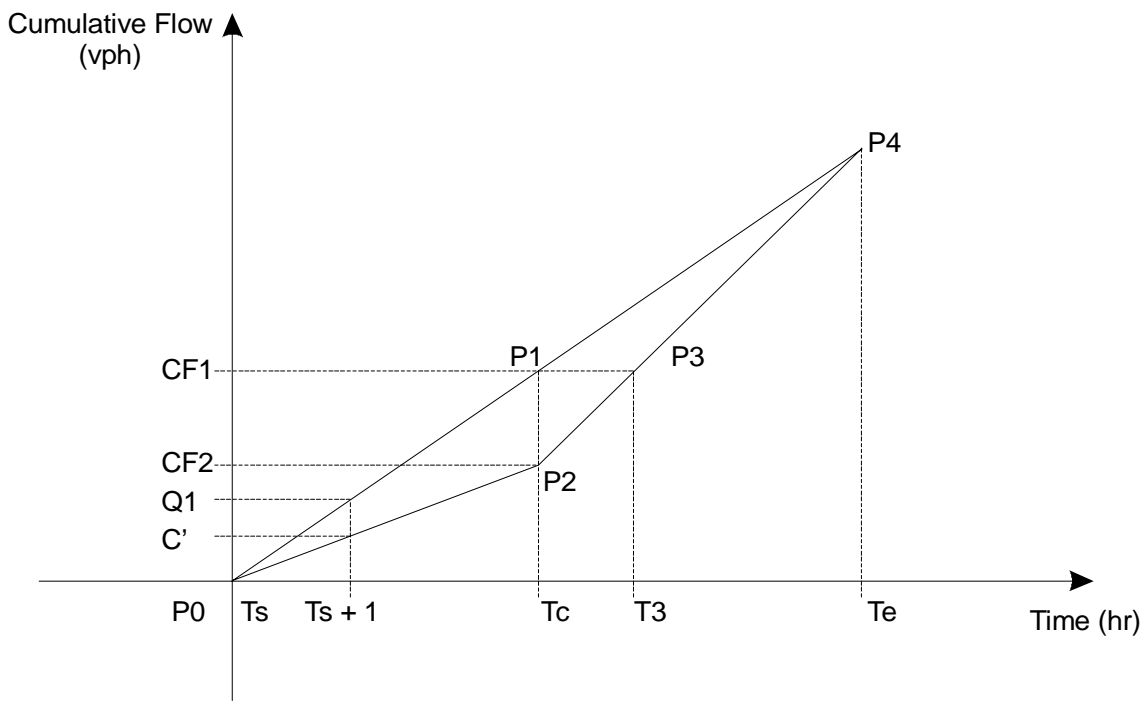

Fig. 7. Cumulative flow vs. time for a highway bottleneck

In Fig. 7, it is assumed that, on a freeway with constant demand Q1, an event occurs at time Ts, reducing the capacity of a section of that freeway from $\mathrm{C}$ to $\mathrm{C}^{\prime}$. Line P0-P4 represents the cumulative demand over the entire period from $\mathrm{Ts}$ to $\mathrm{Te}$ in which there is congestion on the freeway due to an event (the slope of this line is Q1). The event ends at time Tc (i.e., the duration of the event is Tc - Ts), and during this period this section of freeway is operating at reduced capacity $\mathrm{C}^{\prime}$. Line P0-P2 represents the cumulative capacity of the bottleneck (the slope of this line is $\mathrm{C}^{\prime}<\mathrm{Q} 1$ ). Once the event ends (time Tc), the freeway 
returns to its normal capacity $\mathrm{C}$ (line $\mathrm{P} 2-\mathrm{P} 4$ represents the cumulative capacity under normal conditions; the slope of this line is $\mathrm{C}>\mathrm{Q} 1)$.

During the period Ts to Tc, the demand exceeds capacity, causing an accumulation of vehicles upstream of the bottleneck as discussed before. When the capacity of the freeway is restored (time Tc), there is a queue with a length given by the difference in the ordinates of points P1 and P2 (i.e., CF1 - CF2). It takes $\mathrm{Te}-\mathrm{Tc}$ hrs to dissipate this queue once capacity is restored to $\mathrm{C} v \mathrm{vp}$. Therefore, the total duration of the event and its effects are calculated as Te - Ts, and the maximum individual delay is given by the difference of the abscissas of points P3 and P1 (i.e., T3 - Tc). It can also be shown that the area enclosed between the cumulative demand line and the cumulative capacity lines gives the total delay. Equations 6 and 7 show the areas of triangles $\mathrm{P} 1-\mathrm{P} 0-\mathrm{P} 2$ and $\mathrm{P} 1-\mathrm{P} 4-\mathrm{P} 2$, respectively, and equation 8 shows the total delay (TD).

$$
\begin{aligned}
& A T_{1}=\frac{\left(Q 1-C^{\prime}\right) \times(T c-T s)^{2}}{2} \\
& A T_{2}=\frac{\left(Q 1-C^{\prime}\right) \times(T c-T s) \times(T e-T c)}{2} \\
& T D=\frac{\left(Q 1-C^{\prime}\right) \times(T c-T s) \times(T e-T s)}{2}
\end{aligned}
$$

Note: The average delay per vehicle can be computed by dividing TD by the total number of vehicles involved (i.e., $\mathrm{Q} 1 \times(\mathrm{Te}-\mathrm{Ts}))$.

If the event is a crash, several actions may be taken to safeguard public safety while the incident is being cleared. This produces a situation in which the reduced capacity of the roadway changes over time. Also, the demand may not be constant during the duration of the incident and its effects. In Fig. 7 and equation 8 , it is assumed that both demand $\mathrm{Q} 1$ and capacity $\mathrm{C}^{\prime}$ are constant over time. Fig. 8 shows a more general case where this assumption has been relaxed.

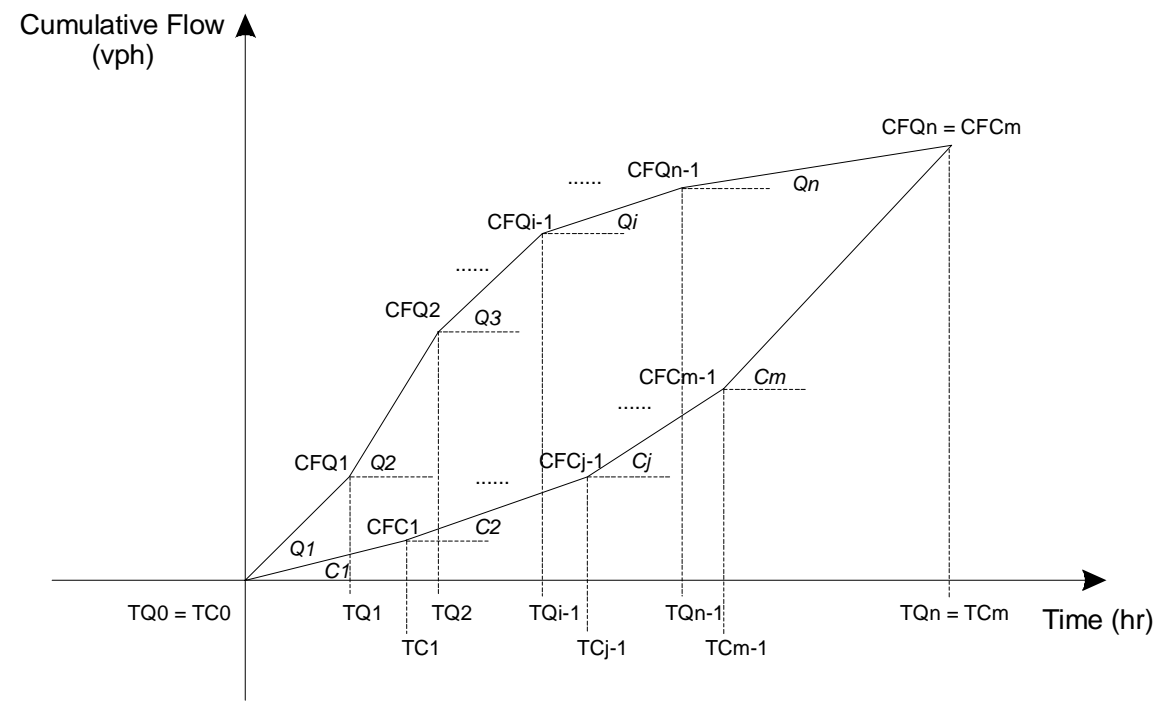

Fig. 8. Cumulative flow vs. time for a highway bottleneck with demand and capacity varying over time 
For example, given an incident that occurs at time $\mathrm{TC}_{0}$ and is cleared at time $\mathrm{TC}_{\mathrm{m}-1}$, events occur during the interval (e.g., arrival of personnel from emergency agencies, removal of affected vehicles to the shoulder, etc.) that affect the capacity of the roadway. In this case, those $\mathrm{m}-1$ events are represented by a starting time $\mathrm{TC}_{\mathrm{j}-1}$, an ending time $\mathrm{TC}_{\mathrm{j}}$, and a capacity level $\mathrm{C}_{\mathrm{j}}$ that is assumed constant during that interval (i.e., the slope of the Cumulative Capacity Flow line in the interval $\left[\mathrm{TC}_{\mathrm{j}-1}, \mathrm{TC}_{\mathrm{j}}\right]$ ). At time $\mathrm{TC}_{\mathrm{m}-1}$, the capacity of the road is restored to normal (i.e., $\mathrm{C}_{\mathrm{m}}=\mathrm{C}$ ).

Thus, during an event and after its clearance, the demand may not be constant. Figure 8 shows a case where the demand has been divided into intervals $\left[\mathrm{TQ}_{\mathrm{i}-1}, \mathrm{TQ}_{\mathrm{i}}\right]$ within which it has a constant value of $\mathrm{Q}_{\mathrm{i}}$ (i.e., the slope of the Cumulative Demand Flow line in the interval [TQ $\left.\mathrm{TQ}_{\mathrm{i}-1}, \mathrm{TQi}\right]$ ). As opposed to the capacity side, it is not known beforehand how many intervals need to be considered for the demand. All of the intervals with constant demand that have starting and ending times within the interval $\left[\mathrm{TC}_{0}=\mathrm{TQ}_{0}\right.$, $\left.\mathrm{TC}_{\mathrm{m}-1}\right]$ must be included. A simple iterative process must be used to determine which constant demand intervals having initial times later than $\mathrm{TC}_{\mathrm{m}-1}$ are to be considered. ${ }^{1}$ This is because point $\left(\mathrm{TQ}_{\mathrm{n}}, \mathrm{CFQ}_{\mathrm{n}}\right) \equiv$ $\left(\mathrm{TC}_{\mathrm{m}}, \mathrm{CFC}_{\mathrm{m}}\right)$ is not known beforehand.

The cumulative demand and capacity at the end of each respective constant interval can be found from equations 9 and 10 .

$$
\begin{gathered}
C F Q_{i}=\sum_{s=1}^{i-1} Q_{s}\left(T Q_{s}-T Q_{s-1}\right) \\
C F C_{j}=\sum_{s=1}^{j-1} C_{s}\left(T C_{s}-T C_{s-1}\right)
\end{gathered}
$$

Assuming that there are $\mathrm{n}$ intervals with constant demand and $\mathrm{m}$ intervals with constant capacity, and considering that $\mathrm{C}_{\mathrm{m}}=\mathrm{C}$, then

$$
T Q_{n}=T C_{m}=\frac{C F C_{m-1}-C F Q_{n-1}+Q_{n} \times T Q_{n-1}-C \times T C_{m-1}+T C_{0} \times\left(Q_{n}-C\right)}{\left(Q_{n}-C\right)}
$$

and the total delay

$$
T D=\frac{1}{2}\left[\sum_{i=1}^{n}\left(C F Q_{i-1} \times T Q_{i}-C F Q_{i} \times T Q_{i-1}\right)-\sum_{j=1}^{m}\left(C F C_{j-1} \times T C_{j}-C F C_{j} \times T C_{j-1}\right)\right]
$$

Note: Another way of computing the delay would be to calculate $S$ from equation 4 as a function of time, given $\mathrm{Q}(\mathrm{t})$ and $\mathrm{C}(\mathrm{t})$ and using the following equation to determine $\mathrm{D}(\mathrm{t})$.

$$
Q(t)=\frac{4 \times C}{J d^{2}} \times D(t) \times[J d-D(t)]
$$

\footnotetext{
${ }^{1}$ For each interval $\mathrm{k}$ with constant demand that starts after time $\mathrm{TC}_{\mathrm{m}-1}$, find the intersection with the line defined by point $\left(\mathrm{TC}_{\mathrm{m}-1}, \mathrm{CFC}_{\mathrm{m}-1}\right)$ and slope $\mathrm{C}_{\mathrm{m}}$. If the intersection is outside the interval $\mathrm{k}$, then go to the next interval with constant demand. If it is inside, then stop.
} 
where $\mathrm{Jd}$ is the "jam density" and C is the capacity of the roadway.

$\mathrm{S}(\mathrm{t})$ would provide the speed at which the queue is growing upstream of the bottleneck. Furthermore, since the density in that area is $\mathrm{D} 4(\mathrm{t})$, it is possible to know the queue length $\mathrm{QL}(\mathrm{t})$ by multiplying $\mathrm{S}(\mathrm{t}) \times$ $\mathrm{D} 4(\mathrm{t})$. The summation of $\mathrm{QL}(\mathrm{t}) \times \mathrm{dt}$ (where $\mathrm{dt}$ is a small discrete interval of time) would give the total delay.

\subsection{DELAY IN THE CONTEXT OF TRAFFIC PATTERNS AND OTHER FACTORS}

The methodology presented in section 2.2 above describes the complex interaction among capacity reduction, demand, and delay factors for a given event. For most of the estimates in this report, this general methodology is applied to each incident on the network, and the capacity loss and delay impacts are summed across area type (including urban area size), highway type, peak period, and (for each peak period) congestion level. These detailed breakdowns of capacity loss and delay present a challenge when trying to (1) compare the relative impacts on different urban area sizes, highway types, and environments and (2) determine the reasonableness of the estimates relative to one another.

A detailed analysis as to why delay estimates vary across city sizes, road types, and time of the day (peak vs. non-peak) is beyond the scope of this study. The amount of vehicle travel (VMT) is certainly a key factor, but other factors such as congestion level and the nature of the events themselves can also affect delay dramatically.

The VMT information in Figure 9 and Table 6 provide a useful context for assessing the order of magnitude of the delay estimates provided for various area, highway, and time-of-day categories presented in this report. However, the reader should also bear in mind that other factors may certainly contribute to differences among various estimates.

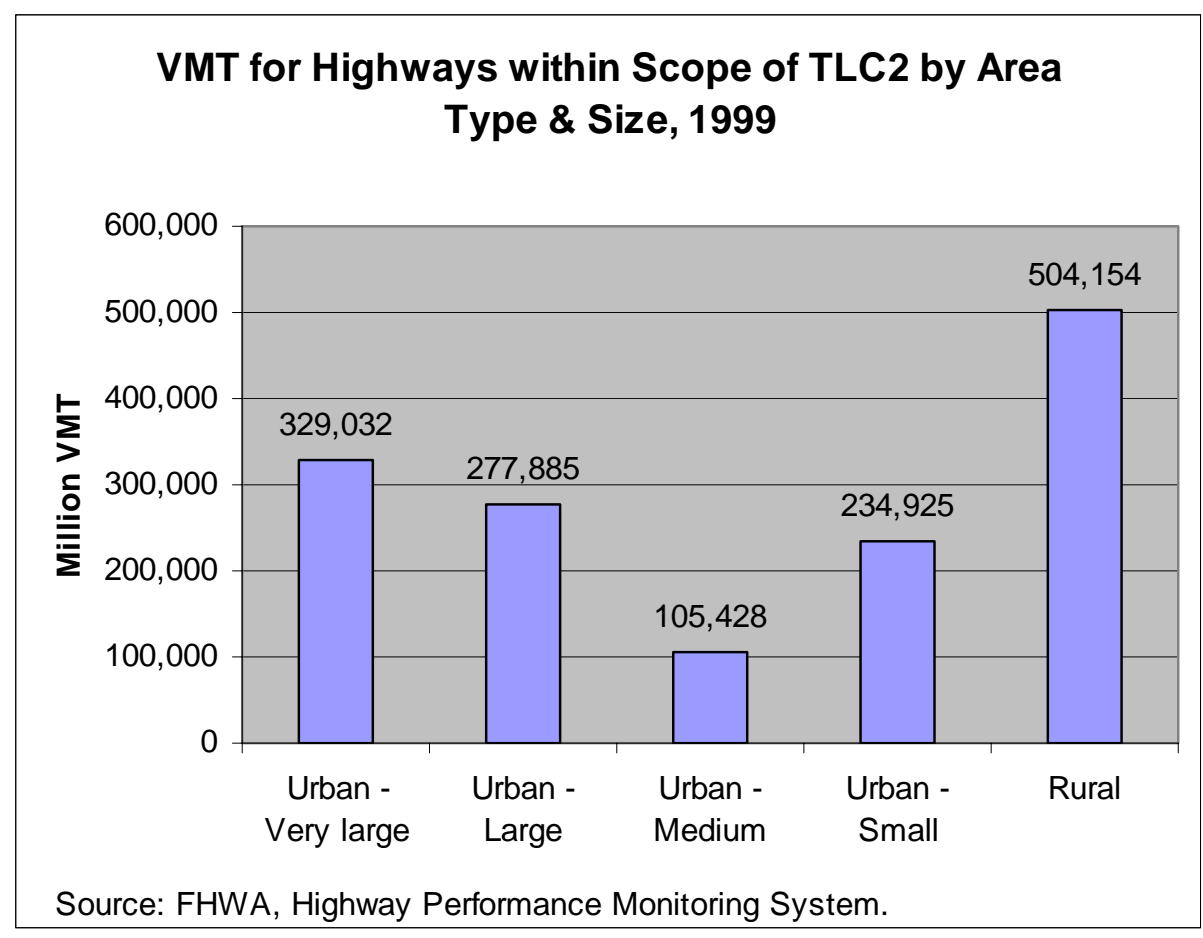

Fig. 9. VMT for highways within scope of TLC2 by area type \& size, 1999 
Table 6. Detailed breakdown of VMT, 1999*

\begin{tabular}{|c|c|c|c|c|}
\hline Highway type & Urban area size & Peak period & Congestion level & Million VMT \\
\hline \multirow{5}{*}{\multicolumn{2}{|c|}{ Very large }} & \multicolumn{3}{|l|}{ Urban } \\
\hline & & \multirow{2}{*}{ Peak } & Congested & 22,345 \\
\hline & & & Not congested & 44,077 \\
\hline & & \multicolumn{2}{|l|}{ Off-peak } & 130,072 \\
\hline & & \multicolumn{2}{|l|}{ Total } & 196,494 \\
\hline \multirow{13}{*}{$\begin{array}{l}\text { Urban freeways \& } \\
\text { expressways }\end{array}$} & \multirow{4}{*}{ Large } & \multirow{2}{*}{ Peak } & Congested & 14,854 \\
\hline & & & Not congested & 45,641 \\
\hline & & \multicolumn{2}{|l|}{ Off-peak } & 117,763 \\
\hline & & \multicolumn{2}{|l|}{ Total } & 178,258 \\
\hline & \multirow{4}{*}{ Medium } & \multirow{2}{*}{ Peak } & Congested & 3,950 \\
\hline & & & Not congested & 17,679 \\
\hline & & \multicolumn{2}{|l|}{ Off-peak } & 41,930 \\
\hline & & \multicolumn{2}{|l|}{ Total } & 63,559 \\
\hline & \multirow{4}{*}{ Small } & \multirow{2}{*}{ Peak } & Congested & 3,883 \\
\hline & & & Not congested & 35,818 \\
\hline & & \multicolumn{2}{|l|}{ Off-peak } & 76,537 \\
\hline & & \multicolumn{2}{|l|}{ Total } & 116,238 \\
\hline & \multicolumn{3}{|l|}{ Total } & 554,549 \\
\hline \multirow{17}{*}{$\begin{array}{l}\text { Urban other } \\
\text { principal arterials }\end{array}$} & \multirow{4}{*}{ Very large } & \multirow{2}{*}{ Peak } & Congested & 9,468 \\
\hline & & & Not congested & 35,340 \\
\hline & & \multicolumn{2}{|l|}{ Off-peak } & 87,730 \\
\hline & & \multicolumn{2}{|l|}{ Total } & 132,538 \\
\hline & \multirow{4}{*}{ Large } & \multirow{2}{*}{ Peak } & Congested & 4,963 \\
\hline & & & Not congested & 28,882 \\
\hline & & Off-peak & & 65,782 \\
\hline & & Total & & 99,627 \\
\hline & & Peak & Congested & 2,168 \\
\hline & Medium & & Not congested & 12,049 \\
\hline & viediumi & Off-peak & & 27,652 \\
\hline & & Total & & 41,869 \\
\hline & & Peak & Congested & 4,297 \\
\hline & Small & Peak & Not congested & 36,146 \\
\hline & Sindan & Off-peak & & 78,244 \\
\hline & & Total & & 118,687 \\
\hline & Total & & & 392,721 \\
\hline & & Peak & Congested & 20,101 \\
\hline Urhan minor colle & & Teak & Not congested & 131,145 \\
\hline 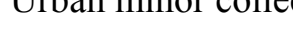 & & Off Peak & & 294,303 \\
\hline & & Total & & 445,549 \\
\hline Urban local & & & & 234,886 \\
\hline All urban & & & & $1,627,705$ \\
\hline
\end{tabular}

\footnotetext{
* Table continued on next page.
} 
Table 6. Detailed breakdown of VMT, 1999 (continued)

\begin{tabular}{|c|c|c|c|}
\hline \multicolumn{4}{|c|}{ Rural } \\
\hline \multirow{4}{*}{ Rural freeways \& expressways } & \multirow{2}{*}{ Peak } & Congested & 2,309 \\
\hline & & Not congested & 86,020 \\
\hline & \multicolumn{2}{|c|}{ Off-peak } & 171,875 \\
\hline & \multicolumn{2}{|l|}{ Total } & 260,204 \\
\hline \multirow{4}{*}{ Rural other principal arterials } & \multirow{2}{*}{ Peak } & Congested & 2,939 \\
\hline & & Not congested & 79,872 \\
\hline & \multicolumn{2}{|c|}{ Off-peak } & 161,139 \\
\hline & \multicolumn{2}{|l|}{ Total } & 243,950 \\
\hline \multirow{4}{*}{ Rural minor arterials \& major collectors } & \multirow{2}{*}{ Peak } & Congested & 2,023 \\
\hline & & Not congested & 125,721 \\
\hline & \multicolumn{2}{|c|}{ Off-peak } & 248,570 \\
\hline & \multicolumn{2}{|l|}{ Total } & 376,314 \\
\hline \multicolumn{3}{|l|}{ Rural minor collectors \& locals } & 183,162 \\
\hline \multicolumn{3}{|l|}{ All rural } & $1,063,631$ \\
\hline \multicolumn{4}{|c|}{ Urban \& Rural } \\
\hline \multicolumn{3}{|l|}{ All urban \& rural } & $2,691,336$ \\
\hline
\end{tabular}

Source: Highway Performance Monitoring System, Federal Highway Administration, USDOT.

* Urban area size categories are based on population: very large - more than 3 million; large - 1 to 3 million; medium 0.5 to 1 million; small - less than 0.5 million.

${ }^{\dagger}$ Peak periods: 6:00 am to 9:30 am and 3:30 pm to 7:00 pm Monday through Friday; all others considered non-peak.

A roadway section is considered congested during the peak periods if its Volume/Service Flow Ratio $(\mathrm{V} / \mathrm{SF})$ is greater than $95 \%$. 


\section{CRASHES}

\subsection{METHODOLOGY}

The concepts described in Section 2 were used to estimate capacity reductions and resulting delays due to vehicle crashes. As discussed before, crashes were defined by the following variables: crash type (fatal, injury, property-damage-only [PDO]), number and type of vehicles involved, the location of the crash, the time of the day and day of the week the crash occurs, and the crash duration. A three-step process was used for estimating delay from vehicle crashes:

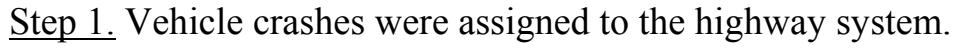

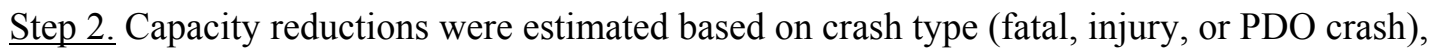
number and type of vehicles involved, the location of the crash, the time of the day the crash occurs, and the crash duration.

Step 3. Delay was estimated based on capacity reduction, vehicle demand, the time of the day and day of the week the crash occurs, and the duration of the capacity reduction.

Due to differences in the characteristics of freeways and principal arterials, capacity reductions and delays for these two highway groups were calculated using slightly different data and methods.

\subsubsection{Assigning Crashes to the Highway Network}

The first step in estimating capacity loss was to assign crashes to a time and location within the national highway network. Two primary data sources were used: the General Estimates System (GES) compiled by the National Highway Traffic Safety Administration (NHTSA) and the Highway Performance Monitoring System (HPMS) maintained by the Federal Highway Administration (FHWA).

General Estimates System (GES): GES data are collected from a nationally representative sample of police-reported crashes, both fatal and non-fatal. To be eligible for the GES sample selection, a police accident report (PAR) must be completed for the crash; the crash must have involved at least one motor vehicle traveling on a highway; and the incident must have resulted in property damage, injury, or death. Data collectors for the GES make weekly visits to approximately 400 police jurisdictions in 60 sites across the United States. They randomly sample about 50,000 PARs every year from these police jurisdictions. These collectors obtain copies of the PARs and send them to a central contractor for coding. No other data is collected beyond the selected PARs - no driver license, vehicle registration, or medical information is obtained. The system began its operation in 1988. At the time of the initial TLC study, the latest available GES data was for 1999.

Highway Performance Monitoring Systems (HPMS): The HPMS, maintained by the FHWA, provides data that reflects the extent, condition, performance, use, and operating characteristics of the nation's highways (U.S. DOT/FHWA 2000). Data collected for 1999 was used for this study. HPMS data compilation is a cooperative effort between FHWA and state highway agencies, local governments, and metropolitan planning organizations (MPOs) working in partnership to collect, assemble, and report necessary information. The physical and operational characteristics of highway facilities on which temporary capacity loss events occurred were drawn from information contained in the HPMS. 
The GES data set was used to assign both fatal and non-fatal crashes to the highway system. GES does not specify the exact location of the crash: it merely specifies the region (Northeast, Mid-West, South, and West), land use type (large central city, suburb, other), general facility type (Interstate or nonInterstate), and number of lanes. Therefore, a Monte Carlo simulation was used to place each crash on a surrogate highway location that would be similar to the environment under which the crash occurred, as described in the GES.

In the initial TLC, the Fatality Analysis Reporting System (FARS) compiled by NHTSA was used to assign fatal crashes to freeways, while GES was used to assign fatal crashes to principal arterials and to assign non-fatal crashes to both freeways and principal arterials. For TLC2, GES data was used to assign all crash types, including fatal crashes on freeways. Although FARS data for fatal crashes is typically believed to be more accurate, it was still necessary to make several assumptions regarding crash location, lane closures, and capacity reduction. Therefore, GES data was used so that a consistent data set, methodology, and assumptions could be used for all crash types. Also, any differences in data quality between GES and FARS should be relatively small compared to the potential error introduced by assumptions used in the methodology.

For crashes on Interstates, the region data was used to narrow down the possible states in which the crash may have taken place. Then, the land use type and number of lanes from GES and the annual average daily traffic (AADT) from HPMS were used in the Monte Carlo simulation to place the crash on the freeway system. ${ }^{2}$ The process for facilities classified as Other Freeways and Expressways was similar. However, since crashes on these roadways are included in the GES "non-Interstate" category with all other non-Interstate highway crashes, a method was used to disaggregate these crashes to more-specific facility types. In this study, crashes were "shared" to different non-Interstate highway types based on vehicle-miles of travel (VMT) by highway class as estimated in the VM-2 table in Highway Statistics (U.S. DOT/FHWA 2001). These crashes were then assigned to the highway network using the same method as for Interstates.

\subsubsection{Estimating Capacity Reductions from Crashes}

\section{Capacity Reduction on Freeways}

Crashes typically produce a loss of capacity on a freeway. This capacity loss depends on the total number of lanes available and the number of lanes affected by the crash. Table 7, adapted from Table 3, was used to determine such capacity losses. For example, a crash causing the closure of one lane on a two-lane highway would reduce the total capacity available (i.e., 2 lanes $\times 2000 \mathrm{pcphpl}$ ) to approximately one-third of its normal value (i.e., $0.320 \times 2$ lanes $\times 2000 \mathrm{pcphpl}$ ) as opposed to one-half (i.e., $0.5 \times 2$ lanes $\times 2000$ pcphpl) as a naive model would predict. $^{3}$

\footnotetext{
${ }^{2}$ The average of the AADT for the freeways was taken for the freeways matching the land use and number of lanes. The Monte Carlo simulation places the incident on a freeway segment that has an AADT close to the average.

${ }^{3} \mathrm{pcphpl}=$ passenger car equivalent $(\mathrm{PCE})$ vehicles per hour per lane. Passenger car equivalent is a measure used to account for the different size and performance of most multi-axle vehicles.
} 
Table 7. Reduced capacity due to freeway crashes (normal capacity $=\mathbf{1 . 0 0 0}$ )

\begin{tabular}{llllll}
\hline \multirow{2}{*}{ Effect of crash } & \multicolumn{5}{c}{ Number of freeway lanes } \\
\cline { 2 - 6 } & 1 & 2 & 3 & 4 & $5+$ \\
\hline Vehicle on shoulder & $0.450^{*}$ & 0.750 & 0.840 & 0.890 & $0.930^{*}$ \\
1 lane blocked & 0.000 & 0.320 & 0.530 & 0.560 & 0.750 \\
2 lanes blocked & N/A & 0.000 & 0.220 & 0.340 & 0.500 \\
3 lanes blocked & N/A & N/A & 0.000 & $0.150^{*}$ & $0.200^{*}$ \\
4 lanes blocked & N/A & N/A & N/A & 0.000 & $0.100^{*}$ \\
\hline
\end{tabular}

*Assumed

The total number of lanes at the location where the crash occurred was obtained from GES. However, data on the number of blocked lanes was not generally available. Lane blockage depends on the type of crash and the number and type of vehicles involved. It was assumed that a fatal or injury crash involving more than one vehicle always results in lane closures (i.e., probability of lane closures $=100$ percent for injury crashes).

To estimate the probability of lane closures when only one vehicle was involved, the study proceeded as follows. First, the probability of a fatal crash not causing lane closures was estimated. From the FARS database for 1998, the total number of fatal crashes that were either located outside of the facility right of way or classified as off-road crashes (1,786 crashes) was estimated. ${ }^{4}$ This number was then divided by the total number of fatal crashes in the database for which the location of the crash was known $(16,542$ crashes out of 16,605). This resulted in a probability of 0.108 (i.e., $1,786 / 16,546=0.108$ ) that no lanes would be closed due to the crash. The probability of lane closures was then computed as $1-0.108=$ 0.892. For injury crashes (i.e., crashes resulting in non-fatal injuries), the same probability of lane closure as for fatal crashes was adopted.

Crash frequency information derived from Giuliano (1989) was used to determine the remaining probabilities of lane closures. Utilizing these frequencies, non-injury crashes had a probability of 0.6 that lanes would be closed (and a complementary probability of 0.4 that no lanes would be closed), while breakdowns were less likely to close lanes (i.e., the probability was 0.154 that a lane would be closed due to a disablement). For property-damage-only (PDO) crashes, these probabilities were overridden if more than three cars or more than one truck was involved in a crash. This study assumed those crashes resulted in lane closures. All fatal crashes involving multiple vehicles were assumed to close lanes. The probability of a fatal crash involving a single vehicle closing a lane was assumed the same as for injury crashes closing a lane. Table 8 summarizes lane closure probabilities.

${ }^{4}$ Although FARS was not used to assign crash locations in TLC2, the data was used to derive the probability of a vehicle involved in a crash to leave the roadway and not block lanes. 
Table 8. Probability of lane closures due to crashes and breakdowns

\begin{tabular}{llcc}
\hline \multicolumn{1}{c}{ Type of crash } & \multicolumn{1}{c}{ Number of vehicles involved } & Lanes closed & No lanes closed \\
\hline Fatal crash & 1 vehicle & 0.892 & 0.108 \\
& More than 1 vehicle & 1.000 & 0.000 \\
Injury crash & 1 vehicle & 0.892 & 0.108 \\
& More than 1 vehicle & 1.000 & 0.000 \\
Property damage only & Less than 3 cars and at most 1 truck & 0.600 & 0.400 \\
& 3 or more cars and/or 2 or more trucks & 1.000 & 0.000 \\
Breakdowns & N/A & 0.154 & 0.846 \\
\hline
\end{tabular}

Table 9 shows the number of lanes closed due to the crash as a function of the number and type of vehicles involved, which is used as a proxy to describe the severity of the crash. It is assumed that, at most, four lanes could be closed due to the crash. This assumption was made since extending the information on capacity losses to cases with more than four lanes closed (see Table 7) could be highly unreliable without further studies (using simulation, for example).

Table 9. Probability distribution of the number of lanes closed

\begin{tabular}{|c|c|c|c|c|c|}
\hline \multirow{2}{*}{$\begin{array}{l}\text { Number of } \\
\text { vehicles involved }\end{array}$} & \multirow{2}{*}{ Type of vehicles involved } & \multicolumn{4}{|c|}{ Lanes closed } \\
\hline & & 1 & 2 & 3 & $4+$ \\
\hline 1 Vehicle & Any type & 0.997 & 0.001 & 0.001 & 0.001 \\
\hline \multirow[t]{2}{*}{2 Vehicles } & 2 cars, or 1 car and 1 truck & 0.950 & 0.048 & 0.001 & 0.001 \\
\hline & 2 trucks & 0.001 & 0.997 & 0.001 & 0.001 \\
\hline \multirow[t]{2}{*}{3 Vehicles } & 3 cars, or 2 cars and 1 truck & 0.500 & 0.450 & 0.049 & 0.001 \\
\hline & 1 car and 2 trucks or 3 trucks & 0.001 & 0.600 & 0.300 & 0.099 \\
\hline More than 3 vehicles & Any type & 0.001 & 0.099 & 0.800 & 0.100 \\
\hline
\end{tabular}

For crashes involving a large number of vehicles (more than 3 ) and occurring on facilities with more than 4 lanes, this assumption may underestimate the delay caused by the crash since more than four lanes could actually be closed. Moreover, the number of lanes closed presented in Table 9 should be a function of the type of crash, since even those crashes involving only one vehicle may result in the closure of all lanes (e.g., a hazardous material spill). In an attempt to capture these types of occurrences, a probability slightly larger than zero (0.001) was given to the closure of any number of lanes for any number and type of vehicles involved.

\section{Capacity Reduction on Principal Arterials}

The procedure used for predicting capacity losses on arterials due to crashes was similar to the one described above for freeways, although some elements differ. Due to a lack of better information, it was assumed that the capacity losses on principal arterials were the same as for crashes on freeways. Thus, it was assumed that the capacity reduction values in Table 5 were also valid for principal arterials.

As explained before, the next step was to determine whether there were lane closures due to the crash. The location distribution for freeways was used as a surrogate for principal arterials. However, since most arterials do not have a shoulder, it was assumed that any crash would produce a lane closure, independent of the type of crash and the number and type of vehicles involved. 
Due to a lack of information, it was assumed that the number of lanes closed was the same as for freeways (see Table 9). It was also assumed, however, that a severe crash on a principal arterial would likely close lanes in both directions of traffic. To account for this, the total number of lanes in both directions was considered when assigning the number of lanes closed. For example, if the model indicated that a crash under consideration closed four lanes on a principal arterial comprised of three lanes in each direction, it was assumed that one direction would be completely closed to traffic, and one lane in the opposite direction would be closed.

\subsubsection{Estimating Delays Due to Crashes}

\section{Delays on Freeways}

Delays were calculated as described in Section 2, "Approach and General Methodology." Three important variables were involved in this computation: (1) the time of the day and day of the week when the crash occurred, (2) its location, and (3) its duration.

Location (rural or urban) together with time of day and day of week were used to determine the demand on the freeway during the interval of time the roadway was affected by the crash. This was done by adjusting the AADT for that location through multipliers obtained from the appropriate demand distribution curve. The study used demand distributions for day of the week and time of the day derived from information collected for four cities: San Antonio, Texas; Milwaukee, Wisconsin; San Diego, California; and Seattle, Washington. ${ }^{5}$ For rural areas, information collected for the state of Tennessee was used. These distributions are shown in the figure below.

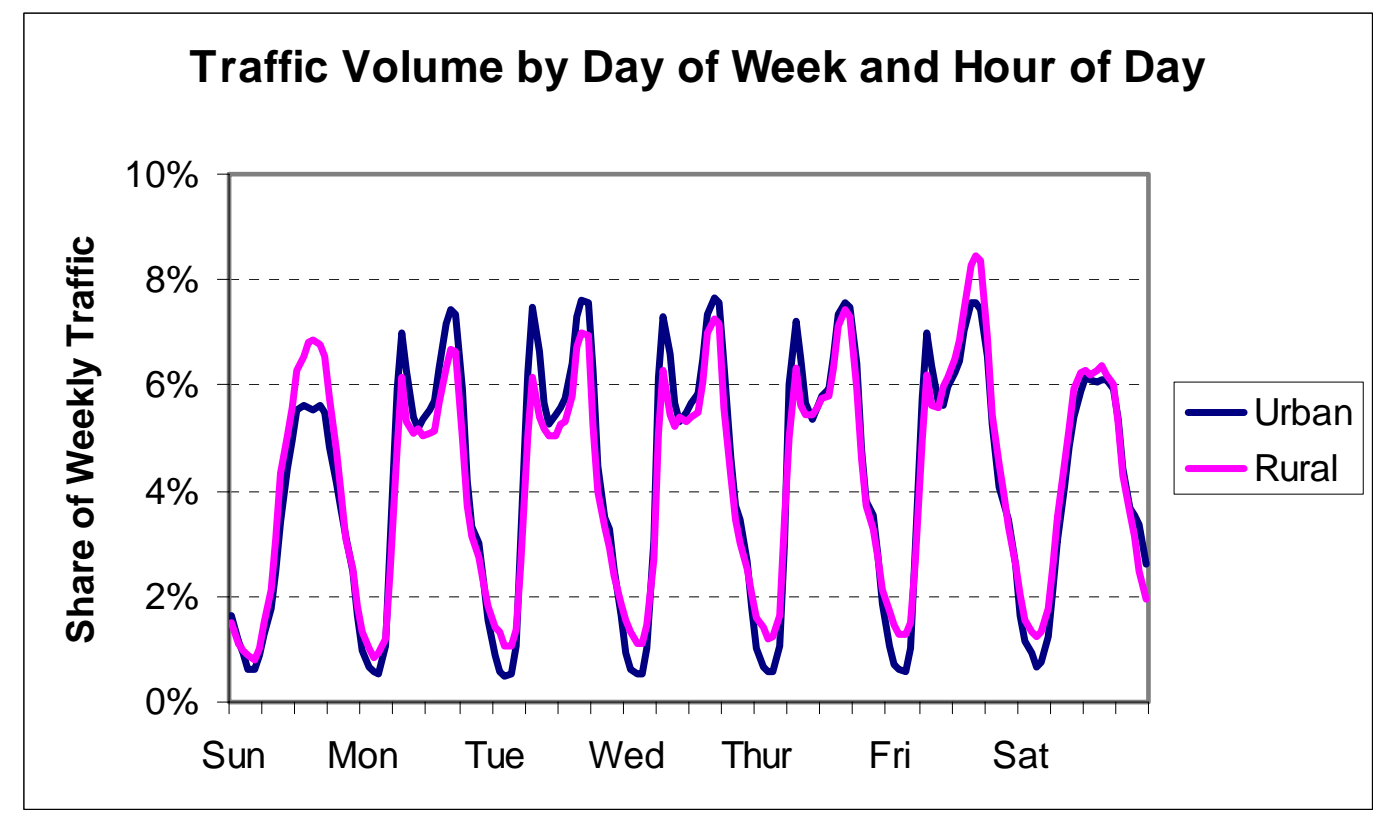

Fig. 10. Traffic volume distributions for urban and rural areas.

\footnotetext{
${ }^{5}$ Data compiled by Oak Ridge National Laboratory (ORNL) from real-time traffic count data from San Antonio, Texas; Milwaukee, Wisconsin; San Diego, California; and Seattle, Washington. For more information see Chin et al., 2000; Chin et al., 1999.
} 
The first step in determining the demand was to adjust the AADT for heavy vehicles. Due to lack of better information, it was assumed that each crash happened on a segment of freeway 0.5 to 1 mile long with a grade of 1 percent. This assumption resulted in a passenger car equivalent (PCE) of 3 for any one percent of trucks in the traffic stream. Information about annual average daily truck volumes (AADTT) on the facility where the crash occurred was obtained from HPMS. To obtain the PCE demand (call it AADT'), the AADT was adjusted by multiplying the estimated number of trucks by three and treating them as passenger vehicles. AADT already contains AADTT, and since the PCE for heavy vehicles was determined to be 3 , then AADT' $=$ AADT $+2 *$ AADTT. The AADT' was then multiplied by 7 to obtain the total volume for the week. The result was then multiplied by the factors (obtained from the urban or rural demand distributions) corresponding to the day of the week and hour of the day when the crash occurred.

Crashes were assumed to be composed of three intervals: (1) the crash detection/arrival-to-scene interval, (2) the remove-to-shoulder interval, and (3) the clearance interval. The crash detection/arrival-to-scene interval is the time that elapses between the actual occurrence of the crash and the time at which the corresponding emergency management (EM) personnel arrive at the location of the crash. During this interval, it was assumed that the vehicles involved were blocking a number of lanes determined using the procedure explained above. For a large percentage of the fatal crashes, the database contained information on the time at which the crash occurred and the time at which the police or other EM personnel arrived at the scene. For those cases, the crash detection/arrival-to-scene time was computed as the difference (in minutes) between these two time points. If any or both of these times were not known, a detection/arrival-to-scene time of 10 minutes was assumed, which is slightly larger than the one reported by Skabardonis et al. (1998).

The remove-to-shoulder interval (RSI) represents the time required to move the vehicles from the roadway to the shoulder. Table 10 shows the remove-to-shoulder duration times as a function of the number and type of vehicles involved in the crash. During this interval, it was assumed that the vehicles involved were blocking a number of lanes determined using the procedure explained above.

Table 10. Interval of time to remove vehicles to shoulders

\begin{tabular}{llcccc}
\hline \multirow{2}{*}{$\begin{array}{l}\text { Number of vehicles } \\
\text { involved }\end{array}$} & Types of vehicles involved & $\begin{array}{c}\text { Time (minutes) } \\
\text { closed }\end{array}$ & $\begin{array}{c}\text { 1 Lane } \\
\text { closed }\end{array}$ & $\begin{array}{c}2 \text { Lanes } \\
\text { closed }\end{array}$ & $\begin{array}{c}\text { 3+ Lanes } \\
\text { closed }\end{array}$ \\
\hline 1 Vehicle & Any type & 0 & 10 & $\infty^{*}$ & $\infty^{*}$ \\
2 Vehicles & 2 cars, or 1 car and 1 truck & 0 & 10 & 15 & $\infty^{*}$ \\
& 2 trucks & 0 & $\infty^{*}$ & $\infty^{*}$ & $\infty^{*}$ \\
3 Vehicles & 3 cars, or 2 cars and 1 truck & 0 & 10 & 15 & $\infty^{*}$ \\
& 1 car and 2 trucks or 3 trucks & 0 & $\infty^{*}$ & $\infty^{*}$ & $\infty^{*}$ \\
More than 3 vehicles & Any type & 0 & $\infty^{*}$ & $\infty^{*}$ & $\infty^{*}$ \\
\hline
\end{tabular}

*Indicates that vehicles are not moved to the shoulder

The crash duration (CD) time represents the time elapsed between the arrival of emergency management personnel to the scene and the time at which the crash was totally cleared (this comprises both the remove-to-shoulder time and the clearance time). The TLC study used information derived from Giuliano (Table 11). 
Table 11. Estimated crash duration (minutes): mean and standard deviation (SD)

\begin{tabular}{l|cccc|cccc}
\hline \multirow{2}{*}{ Incident type } & \multicolumn{3}{|c|}{ No lanes closed } & \multicolumn{4}{c}{ Lanes closed } \\
& Mean & SD & Mean & SD & Mean & SD & Mean & SD \\
\hline Injury crash & 47 & 29 & 62 & 40 & 54 & 28 & 66 & 58 \\
Non-injury crash & 41 & 24 & 47 & 24 & 38 & 22 & 66 & 41 \\
Breakdown & 29 & 22 & 30 & 24 & 14 & 11 & 18 & 22 \\
\hline
\end{tabular}

Knowing the type of crash and whether or not the crash resulted in lane closure (which was previously determined to assess the loss of capacity), the table gives the mean of the crash duration and its standard deviation. Table 12 below, derived from Table 11, shows these mean duration times. (Note: only the means are used in this version of this study.)

Table 12. Estimated crash duration (in minutes)

\begin{tabular}{l|c|c|c|c}
\hline \multirow{2}{*}{ Crash type } & \multicolumn{2}{|c|}{$\begin{array}{c}\text { Daytime } \\
(6 \mathrm{am}-6 \mathrm{pm})\end{array}$} & \multicolumn{2}{c}{$\begin{array}{c}\text { Nighttime } \\
(6 \mathrm{pm}-6 \mathrm{am})\end{array}$} \\
\cline { 2 - 5 } & No lanes closed & Lanes closed & No lanes closed & Lanes closed \\
\hline Injury crash & 47 & 62 & 54 & 66 \\
Non-injury crash & 41 & 47 & 38 & 66 \\
Breakdown & 29 & 30 & 14 & 18 \\
\hline
\end{tabular}

The remove-to-shoulder interval (RSI) is assumed part of the CD interval. That is, for those cases where $\mathrm{RSI}<=15$ minutes, it was assumed that, during the interval CD - RSI, the vehicles involved in the crash where located on the shoulder of the freeway, producing a capacity loss obtained from the first row of Table 7. Otherwise, it was assumed that, during the entire interval CD, the vehicles were blocking 1 or 2 lanes, as determined above, producing the corresponding capacity loss from Table 7.

\section{Delays on Principal Arterials}

Delay on principal arterials was estimated using the same method used for freeways, although additional assumptions were necessary. Principal arterial traffic demand was adjusted using the multipliers for time of day and day of the week, just as it was for freeways. Due to the lack of better information, the same multipliers used for freeway demand were used for principal arterials.

Since it was assumed that principal arterials do not have shoulders, crashes on those types of facilities were composed of just one interval, rather than the three intervals used for crashes on freeways. In most cases, the length of this interval was assumed equal to the duration of the crash (see Table 12) plus another 10 minutes to account for the detection of the crash. However, there is one exception to this rule. As previously stated, the freeway lane-location distribution was used to locate non-fatal crashes on principal arterials. However, "property damage only" (PDO) crashes (i.e., non-fatal, non-injury) involving less than four vehicles and assumed to end up on the shoulder in the location distribution were assigned a much smaller duration. A duration of 15 minutes was assumed for this kind of crash (10 minutes for the arrival of police to the scene and another 5 minutes to move the vehicles out of the way).

The delays were calculated using essentially the same approach used for freeways, with the following differences: First, an ideal capacity of 1,600 vphpl was assumed. Second, the green time percentage for the principal arterial was generated from a uniform distribution with lower and upper bounds of 50 and 70 
percent, respectively. ${ }^{6}$ Since a principal arterial should get a green light at least half of the time, a lower bound of 50 percent was assumed. The upper bound of 70 percent green time was also based on assumption. Third, it was assumed that the principal arterials form a grid with separation $L$ uniformly distributed in the interval $[0.5,3.0]$ miles. It was further assumed that the crash had the same probability of being located anywhere along the arterial under consideration. Calling $d$ the distance from the immediate upstream transversal arterial to the location at which the crash occurred $(d<=L)$, it was assumed that the queue due to the crash could not be longer than $d$, since traffic would likely divert at the transversal arterial upstream of the crash. In effect, the size of each queue on an arterial was limited by truncating it at a length equal to $d$. This represents traffic diversion in an arterial network grid. Although the traffic diverting would not experience any delay due to the crash, the vehicles would incur a longer trip and, in consequence, longer travel times. However, since the effects of re-routing were beyond the scope of the present study, those longer travel times were not computed as part of the delays due to crashes on principal arterials.

\subsection{RESULTS}

The TLC2 study estimates that, in 1999, approximately 3.3 million crashes on freeways and principal arterials caused temporary capacity reductions of about 3.3 billion vehicles and over 1.7 billion vehiclehours of delay (Fig. 11, Table 13). Crashes on freeways caused nearly three times as much delay as those on principal arterials. Freeway crashes produced around 1.2 billion vehicle-hours of delay ( 73 percent), while crashes on principal arterials accounted for around 459 million (27 percent). The average delay caused by each freeway crash (1.2 thousand vehicle-hours) was about six times more than the average for each principal arterial crash (197.5 vehicle-hours). Similarly, average delay per crash was 50 percent higher for fatal crashes than for non-fatal crashes. Still, due to the higher frequency of non-fatal crashes and the fact that fatal crashes typically occur during non-congested conditions, non-fatal crashes accounted for about 99 percent of delay from all crashes.

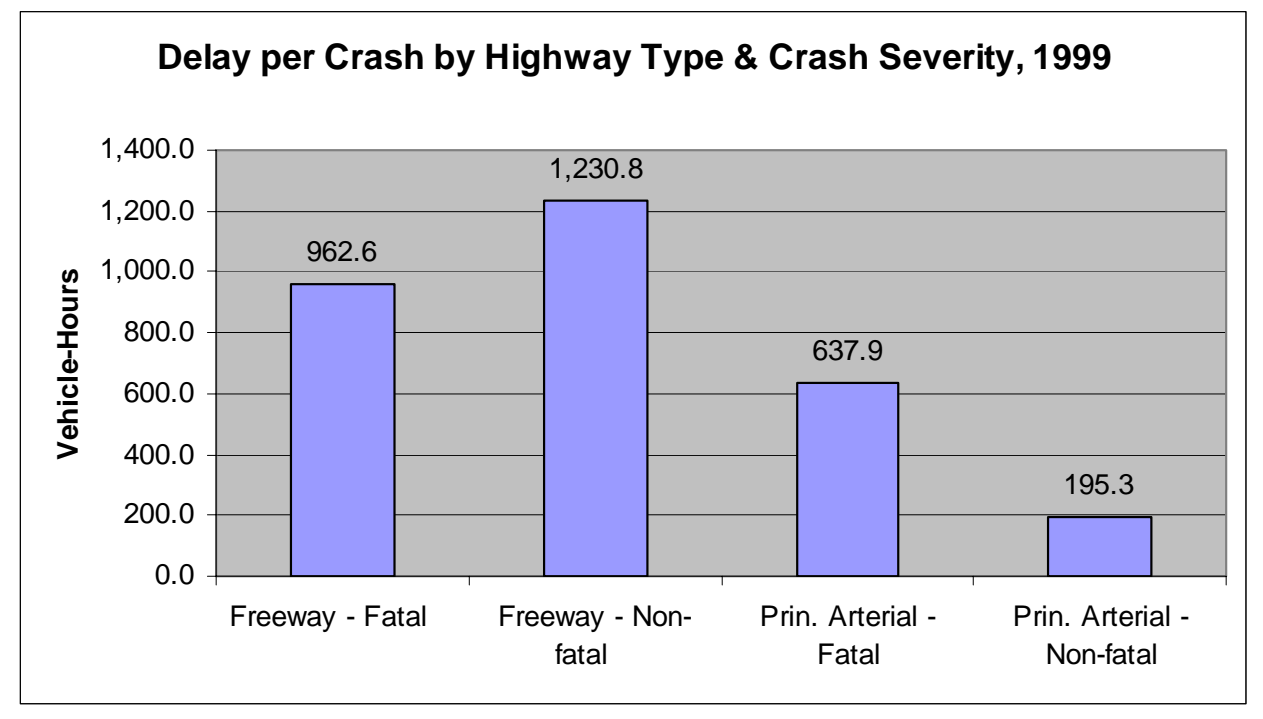

Fig. 11. Delay per crash by severity and highway type

\footnotetext{
${ }^{6}$ Since the ideal capacity represents the flow on uncontrolled links, the green time percentage is used to adjust the capacity to account for the fact that traffic is stopped part of the time.
} 
Table 13. Estimated capacity reductions and delay due to fatal and non-fatal crashes on freeways and principal arterials, 1999

\begin{tabular}{|c|c|c|c|c|c|}
\hline Highway type & Severity & Crashes & $\begin{array}{c}\text { Capacity lost } \\
(1,000 \text { vehicles })\end{array}$ & $\begin{array}{c}\text { Delay } \\
(1,000 \text { veh-hours })\end{array}$ & $\begin{array}{c}\text { Average } \\
\text { delay/crash } \\
\text { (veh-hours) }\end{array}$ \\
\hline \multirow{3}{*}{ Freeways } & Fatal & 6,529 & $12,978.6$ & $6,284.5$ & 962.6 \\
\hline & Non-fatal & 984,934 & $1,376,188.4$ & $1,212,291.8$ & $1,230.8$ \\
\hline & All & 991,463 & $1,389,167$ & $1,218,576$ & $1,229.1$ \\
\hline \multirow{3}{*}{ Principal arterials } & Fatal & 11,689 & $17,558.3$ & $7,456.8$ & 637.9 \\
\hline & Non-fatal & $2,313,405$ & $1,878,329.7$ & $451,864.1$ & 195.3 \\
\hline & All & $2,325,094$ & $1,895,888.0$ & $459,320.9$ & 197.5 \\
\hline \multirow{3}{*}{$\begin{array}{l}\text { Freeways \& } \\
\text { principal arterials }\end{array}$} & Fatal & 18,218 & 30,537 & 13,741 & 754.3 \\
\hline & Non-fatal & $3,298,339$ & $3,254,518$ & $1,664,156$ & 504.5 \\
\hline & All & $3,316,557$ & $3,285,055$ & $1,677,897$ & 505.9 \\
\hline
\end{tabular}


Table 14. Capacity reductions \& delays from fatal crashes on freeways and principal arterials, 1999

\begin{tabular}{|c|c|c|c|c|c|c|}
\hline Highway type & $\begin{array}{c}\text { Urban area } \\
\text { size* }\end{array}$ & $\begin{array}{l}\text { Peak } \\
\text { period }^{\dagger}\end{array}$ & $\begin{array}{c}\text { Congestion } \\
\text { level }^{\ddagger}\end{array}$ & $\begin{array}{l}\text { No. of } \\
\text { crashes }\end{array}$ & $\begin{array}{c}\text { Capacity } \\
\text { reduction } \\
(1,000 \text { vehs }) \\
\end{array}$ & $\begin{array}{c}\text { Delay } \\
(1,000 \text { veh- } \\
\text { hrs }) \\
\end{array}$ \\
\hline \multirow{13}{*}{$\begin{array}{l}\text { Urban freeways } \\
\text { and expressways }\end{array}$} & \multirow{3}{*}{ Very large } & \multirow{2}{*}{ Peak } & Congested & $-\S$ & -\$ & $-\S$ \\
\hline & & & Not congested & 67 & 109.3 & 630.1 \\
\hline & & \multicolumn{2}{|l|}{ Off-peak } & 1,496 & $2,987.1$ & 210.1 \\
\hline & \multirow{3}{*}{ Large } & \multirow{2}{*}{ Peak } & Congested & 319 & 521.4 & 3.4 \\
\hline & & & Not congested & 518 & 906.8 & $1,233.4$ \\
\hline & & \multicolumn{2}{|l|}{ Off-peak } & 1,064 & $1,866.5$ & $3,971.3$ \\
\hline & \multirow{3}{*}{ Medium } & \multirow{2}{*}{ Peak } & Congested & $-\$$ & $-\S$ & $-\S$ \\
\hline & & & Not congested & 110 & 184.7 & 5.9 \\
\hline & & \multicolumn{2}{|l|}{ Off-peak } & 503 & $1,204.9$ & 22.3 \\
\hline & \multirow{3}{*}{ Small } & \multirow{2}{*}{ Peak } & Congested & $-\$$ & — & — \\
\hline & & & Not congested & 242 & 390.8 & 1.1 \\
\hline & & \multicolumn{2}{|l|}{ Off-peak } & 944 & $2,435.6$ & 18.1 \\
\hline & \multicolumn{3}{|l|}{ Total } & 5,263 & $10,607.0$ & $6,095.8$ \\
\hline \multirow{13}{*}{$\begin{array}{l}\text { Urban other } \\
\text { principal arterials }\end{array}$} & \multirow{3}{*}{ Very large } & \multirow{2}{*}{ Peak } & Congested & 2 & 4.4 & 0.01 \\
\hline & & & Not congested & 452 & 584.8 & $1,130.5$ \\
\hline & & \multicolumn{2}{|l|}{ Off-peak } & 1,138 & $1,934.6$ & 529.0 \\
\hline & \multirow{3}{*}{ Large } & \multirow{2}{*}{ Peak } & Congested & $-\$$ & $-\S$ & $-\S$ \\
\hline & & & Not congested & 194 & 302.0 & 118.5 \\
\hline & & \multicolumn{2}{|l|}{ Off-peak } & 1,790 & $3,016.6$ & $3,054.9$ \\
\hline & \multirow{3}{*}{ Medium } & \multirow{2}{*}{ Peak } & Congested & $-\S$ & — & — \\
\hline & & & Not congested & 45 & 103.6 & 65.3 \\
\hline & & \multicolumn{2}{|l|}{ Off-peak } & 267 & 454.0 & 27.6 \\
\hline & \multirow{3}{*}{ Small } & \multirow{2}{*}{ Peak } & Congested & 136 & 240.2 & 0.2 \\
\hline & & & Not congested & 264 & 369.3 & 165.4 \\
\hline & & \multicolumn{2}{|l|}{ Off-peak } & 2,610 & $3,947.7$ & $1,936.6$ \\
\hline & \multicolumn{3}{|l|}{ Total } & 6,898 & $10,957.0$ & $7,028.0$ \\
\hline & & & Congested & 8 & 13.3 & 106.4 \\
\hline Rural freewavs & & Peak & Not congested & 300 & 542.3 & 49.5 \\
\hline Kural ireeways & & Off-peak & & 958 & $1,816.0$ & 32.8 \\
\hline & & Total & & 1,266 & $2,371.6$ & 188.8 \\
\hline & & Peak & Congested & $-\S$ & $-\$$ & $-\S$ \\
\hline Rural other nrincin & al arterials & Peak & Not congested & 1,303 & $1,509.7$ & 247.5 \\
\hline 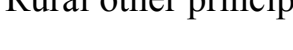 & al allentals & Off-peak & & 3,488 & $5,091.7$ & 181.3 \\
\hline & & Total & & 4,791 & $6,601.4$ & 428.8 \\
\hline Total & & & & 18,218 & $30,537.0$ & $13,741.3$ \\
\hline
\end{tabular}

* Urban area size categories are based on population: very large - more than 3 million; large - 1 to 3 million; medium 0.5 to 1 million; small - less than 0.5 million.

${ }^{\dagger}$ Peak periods: 6:00 am to 9:30 am and 3:30 pm to 7:00 pm Monday through Friday; all others considered non-peak.

*A roadway section is considered congested during the peak periods if its Volume/Service Flow Ratio (V/SF) is greater than $95 \%$.

$\S$ The GES data contain no fatal crashes for this urban area type, time period, and traffic condition. Therefore, capacity reduction and delay could not be extrapolated for this cell within the table. While it is possible that a crash (or crashes) did occur under this condition, the probability of such a crash is very low. 
Table 15. Capacity reductions \& delays from non-fatal crashes on freeways \& principal arterials, 1999

\begin{tabular}{|c|c|c|c|c|c|c|}
\hline Highway type & $\begin{array}{c}\text { Urban area } \\
\text { size* }\end{array}$ & $\begin{array}{l}\text { Peak } \\
\text { period }^{\dagger}\end{array}$ & $\begin{array}{c}\text { Congestion } \\
\text { level }^{\ddagger}\end{array}$ & $\begin{array}{l}\text { No. of } \\
\text { crashes }\end{array}$ & $\begin{array}{c}\text { Capacity } \\
\text { reduction } \\
(1,000 \text { vehs })\end{array}$ & $\begin{array}{c}\text { Delay } \\
(1,000 \text { veh- } \\
\text { hrs })\end{array}$ \\
\hline \multirow{13}{*}{$\begin{array}{l}\text { Urban freeways } \\
\text { and expressways }\end{array}$} & \multirow{3}{*}{ Very large } & \multirow[t]{2}{*}{ Peak } & Congested & 25,280 & $36,151.6$ & $96,559.6$ \\
\hline & & & Not congested & 52,629 & $78,480.1$ & $191,823.1$ \\
\hline & & \multicolumn{2}{|l|}{ Off-peak } & 181,591 & $270,299.0$ & $380,797.7$ \\
\hline & \multirow{3}{*}{ Large } & \multirow[t]{2}{*}{ Peak } & Congested & 17,608 & $23,029.8$ & $49,277.9$ \\
\hline & & & Not congested & 60,222 & $79,320.8$ & $122,497.5$ \\
\hline & & \multicolumn{2}{|l|}{ Off-peak } & 154,149 & $207,720.4$ & $243,336.3$ \\
\hline & \multirow{3}{*}{ Medium } & \multirow[t]{2}{*}{ Peak } & Congested & 2,485 & $4,556.3$ & $9,907.2$ \\
\hline & & & Not congested & 20,877 & $28,789.7$ & $20,927.4$ \\
\hline & & \multicolumn{2}{|l|}{ Off-peak } & 51,901 & $68,989.8$ & $48,582.4$ \\
\hline & \multirow{3}{*}{ Small } & \multirow[t]{2}{*}{ Peak } & Congested & 7,487 & $10,002.3$ & 728.6 \\
\hline & & & Not congested & 64,347 & $89,322.6$ & $12,847.0$ \\
\hline & & \multicolumn{2}{|l|}{ Off-peak } & 162,089 & $220,861.8$ & $18,828.1$ \\
\hline & \multicolumn{3}{|l|}{ Total } & 800,665 & $1,117,524.2$ & $1,196,112.8$ \\
\hline \multirow{13}{*}{$\begin{array}{l}\text { Urban other } \\
\text { principal arterials }\end{array}$} & \multirow{3}{*}{ Very large } & \multirow[t]{2}{*}{ Peak } & Congested & 32,364 & $28,087.6$ & $21,157.9$ \\
\hline & & & Not congested & 81,677 & $66,987.2$ & $38,599.1$ \\
\hline & & \multicolumn{2}{|c|}{ Off-peak } & 260,347 & $243,968.0$ & $79,862.5$ \\
\hline & \multirow{3}{*}{ Large } & \multirow[t]{2}{*}{ Peak } & Congested & 13,013 & $10,817.6$ & $10,883.9$ \\
\hline & & & Not congested & 69,981 & $53,249.0$ & $36,120.7$ \\
\hline & & \multicolumn{2}{|l|}{ Off-peak } & 181,586 & $158,719.9$ & $58,501.1$ \\
\hline & \multirow{3}{*}{ Medium } & \multirow[t]{2}{*}{ Peak } & Congested & 5,610 & $4,050.9$ & $2,778.3$ \\
\hline & & & Not congested & 26,015 & $20,194.1$ & $8,262.5$ \\
\hline & & \multicolumn{2}{|l|}{ Off-peak } & 78,455 & $72,071.2$ & $15,753.4$ \\
\hline & \multirow{3}{*}{ Small } & \multirow[t]{2}{*}{ Peak } & Congested & 17,738 & $12,573.2$ & $8,125.3$ \\
\hline & & & Not congested & 147,352 & $107,952.3$ & $28,279.5$ \\
\hline & & \multicolumn{2}{|l|}{ Off-peak } & 410,144 & $344,304.8$ & $59,197.3$ \\
\hline & \multicolumn{3}{|l|}{ Total } & $1,324,282$ & $1,122,975.9$ & $367,521.5$ \\
\hline & & Peak & Congested & 1,144 & $1,543.6$ & 31.4 \\
\hline & & & Not congested & 61,870 & $84,717.4$ & $5,563.6$ \\
\hline & & Off-peak & & 121,255 & $172,403.3$ & $10,583.9$ \\
\hline Rural freeways & & Total & & 184,269 & $258,664.3$ & $16,178.9$ \\
\hline & & Peak & Congested & 9,632 & $8,341.0$ & $2,457.8$ \\
\hline Rural other & & & Not congested & 288,645 & $196,822.3$ & $30,463.1$ \\
\hline principal arterials & & Off-peak & & 690,846 & $550,190.4$ & $51,421.7$ \\
\hline & & Total & & 989,123 & $755,353.7$ & $84,342.6$ \\
\hline Total & & & & $3,298,339$ & $3,254,518.1$ & $1,664,155.9$ \\
\hline
\end{tabular}

* Urban area size based on population: very large - more than 3 million; large - 1 to 3 million; medium 0.5 to 1 million; small - less than 0.5 million.

${ }^{\dagger}$ Peak periods: 6:00 am to 9:30 am and 3:30 pm to 7:00 pm Monday through Friday; all others considered non-peak.

A roadway section is considered congested during the peak periods if its Volume/Service Flow Ratio $(\mathrm{V} / \mathrm{SF}$ ) is greater than $95 \%$. 


\subsection{RELIABILITY}

\subsubsection{Methodology}

The methodology can be divided into three parts: assigning crashes to the network using a Monte Carlo simulation, estimating capacity loss, and estimating delay.

It is believed that the Monte Carlo simulation method described in section 3.1.1 was a fairly reliable method for assigning crashes to highway links similar to those on which the crash actually occurred. As a limited test of variability, capacity reduction and delay were generated for two datasets, each consisting of ten thousand assigned crash locations. The results for these two data sets were similar. While this is a limited indicator of reliability, it is encouraging.

To estimate capacity loss, capacity reduction multipliers were obtained from the literature or estimated when not available - the reliability of these data and assumptions are discussed below. To estimate delay, the TLC study used standard, well-established procedures and methods derived from traffic flow theory as presented in the Highway Capacity Manual (TRB 1985).

The confidence level of the methodology used here to determine capacity losses and delays due to fatal crashes depends on the type of facility where the accident occurred. For freeways with up to five lanes where at most two lanes are closed, both methodologies can be classified as having a high degree of confidence. For freeways with more lanes (either total and/or closed), the computation of the delays retains a high level of confidence, but the capacity reduction estimates become more unreliable since they are outside the boundaries of the studies from which this information was collected. For arterials and other facilities with unrestricted access, both methodologies can be qualified as having a medium degree of confidence.

\subsubsection{Data \& Key Assumptions}

Crash Locations and Characteristics: The General Estimates System (GES) data used to assign crashes and crash characteristics to the highway network is based on a random sample of about 50,000 PARs every year from about 400 police jurisdictions. It is widely used as a data source for crash-related studies and is qualified as having a high degree of confidence.

Traffic Demand: The traffic demand data is accorded a low level of confidence since it was derived using Annual Average Daily Traffic (AADT) information from HPMS along with time of day and day of week distributions based on freeway data from only four cities and one state. Because of the high impact that demand can have on the computation of delays, further research in this area would need to be performed in future versions of this study.

Probability of Lane Closure: The data and assumptions used for determining lane closure probabilities are classified as having a high degree of confidence for freeway crashes but a lower degree of confidence for arterial crashes.

Number of Lanes Blocked by the Event: The probability distribution of the number of lanes closed by severity of the crash was created based on engineering judgment - number and type of vehicles involved in the crash were used as a proxy to describe its severity. Due to the lack of resources to corroborate the resulting distribution, the data is assigned a medium to low degree of confidence.

Surface Street Characteristics: For principal arterials, the ideal lane capacity, the traffic signal green ratio along the arterial, the geometry of the network (i.e., the size of the arterial grid or separation between 
transversal major arterials), and the location of the crash along the arterial segment in relationship to the closest upstream major transversal arterial were based on engineering judgment. These assumptions can be qualified as having a medium degree of confidence.

Duration of the Event: Crash durations were based on detection and clearance times obtained from several papers that studied freeway incidents, as well as assumptions based on engineering judgment. Assumptions regarding crash duration are accorded a high degree of confidence for freeway crashes.

They are accorded a medium to low level for arterial crashes since the studies on which the assumptions were based included freeways only. 


\section{BREAKDOWNS}

\subsection{METHODOLOGY}

Capacity reductions and delays due to vehicle breakdowns were estimated using a method similar to the one used for crashes. However, since national-level data on breakdowns was not available, additional assumptions were necessary to estimate the annual number of breakdowns, the time they occurred, and their location on the national highway network. The methodology can be summarized into the following steps:

Step 1. The total number of vehicle breakdowns was estimated.

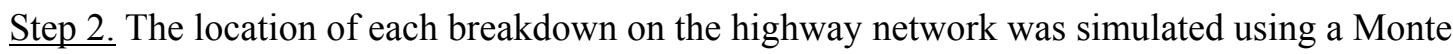
Carlo simulation method based on the VMT on each segment.

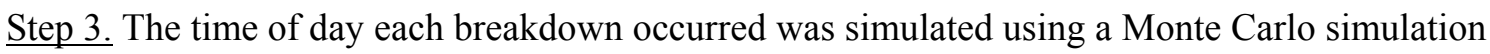
method based on hourly vehicle counts.

Step 4. The location of the vehicle on each selected segment was simulated (e.g., right-hand shoulder, left-hand shoulder, right-most lane, etc.).

Step 5. The capacity reduction due to each breakdown was based on the characteristics of the selected highway segment and the location of the vehicle on the segment (e.g., right-hand shoulder, left-hand shoulder, right-most lane, etc.).

Step 6. Delay was estimated based on capacity reduction, vehicle demand on the segment, and the duration of the capacity reduction.

These steps are described in more detail in the paragraphs below.

\subsubsection{Estimating the Total Number of Breakdowns}

The total number of breakdowns was estimated based on the number of crashes on the highway system. Studies in the literature and data collected from cities with freeway service patrols showed that the ratio of breakdowns to crashes on freeways was roughly eight breakdowns per every crash. Thus, it was assumed that the total number of breakdowns in the nation would be equal to eight times the number of crashes. As described in the sections that follow, temporal and location characteristics were assigned to breakdowns based on traffic volume and VMT.

The ratio of breakdowns to crashes was based on a study by the American Trucking Association (ATA) and Cambridge Systematics, Inc. (1991). In this study, ATA and Cambridge Systematics collected data from Freeway Service Patrols and other agencies that collected crash and breakdown data. They analyzed this data and produced estimates of the percentage of incidents classified as disablements, crashes, and other events (e.g., clearing debris). These shares were further broken down by whether or not they blocked lanes. The study also estimated the average incident duration and the vehicle-hours of delay caused by each type. The ratio of crashes to breakdowns given in the Cambridge Systematics study was comparable to the ratios observed in both the Giuliano (1989) and Skabardonis et al. (1998) studies. 


\subsubsection{Assigning Each Breakdown to a Location in the Highway Network}

Breakdowns were assigned a location within the highway network using a Monte Carlo simulation method. The probability of a breakdown being assigned to a given segment in HPMS was dependent upon the VMT for that segment. VMT was calculated as the product of the segment's length and its AADT volume.

\subsubsection{Assigning Temporal Characteristics to Each Breakdown}

A Monte Carlo simulation was used to assign a day of the week and time of the day to each breakdown. Each hour of the week was assigned a separate bin whose size was dependent upon the traffic volume for that time based on hourly traffic volumes. For breakdowns assigned to urban highways, hourly traffic volume distributions taken from four cities (San Antonio, Texas; Milwaukee, Wisconsin; San Diego, California; and Seattle, Washington) were used in the Monte Carlo simulation (see footnote 5 on page 21). For breakdowns assigned to rural areas, hourly traffic volume distributions taken from the state of Tennessee (T. DOT) were used.

\subsubsection{Assigning Each Breakdown a Location within the Selected Link}

Each breakdown was assigned a location along the link on which it was placed. This includes the lane, shoulder, or other location at which the vehicle came to rest. Locations on freeways were based on statistics in the literature and from data provided by a few highway service patrols. Principal arterial breakdowns were assumed to have characteristics similar to breakdowns on freeways, except it was assumed that they either came to rest in a lane or were able to get the vehicle to a parking area or side street. It was also assumed that most vehicles breaking down on principal arterials were able to get off the principal arterial ( 85 percent) onto a side street or into a parking area.

\subsubsection{Estimating Capacity Reduction}

Capacity reductions were estimated using a methodology similar to the one used for crashes.

\subsubsection{Estimating Delay}

Delay was estimated using a methodology similar to the one used for crashes.

\subsection{RESULTS}

This study estimates that, in 1999, over 27 million vehicle breakdowns occurred on freeways and principal arterials, reducing capacity by nearly 7.5 billion vehicles and causing over 440 million vehiclehours of delay (Table 16). By comparison, crashes caused an estimated 1.7 billion vehicle-hours of delay, nearly four times as much.

This study estimates that breakdowns typically caused only 15.9 vehicle-hours of delay per incident, while crashes caused 505.9 vehicle-hours - over thirty times more delay per occurrence. This is primarily because drivers are usually able to get disabled vehicles off the highway onto the shoulder, a parking area, or a side street with less traffic. Though estimates vary somewhat, studies in the literature and statistics from data sets indicate that approximately 80 percent of vehicle breakdowns do not block highway lanes. This reduces the amount of bottleneck delay significantly although some slowdown from rubbernecking or a vehicle's proximity to traffic lanes can be expected. 
Table 16. Estimated breakdowns and resulting capacity reduction and delay by highway type, 1999

\begin{tabular}{l|l|l|l|l}
\hline \multicolumn{1}{c|}{ Highway type } & Breakdowns & $\begin{array}{c}\text { Capacity } \\
\text { reduction } \\
(1,000 \text { vehs })\end{array}$ & $\begin{array}{c}\text { Delay } \\
(1,000 \text { veh-hrs })\end{array}$ & $\begin{array}{c}\text { Delay per } \\
\text { breakdown } \\
\text { (veh-hrs) }\end{array}$ \\
\hline Urban freeways and expressways & $5,779,862$ & $4,209,866.3$ & $12,130.0$ & 2.1 \\
\hline Urban other principal arterials & $11,892,388$ & $924,006.6$ & $332,192.7$ & 27.9 \\
\hline Rural freeways & $2,707,087$ & $1,825,282.2$ & 428.2 & 0.2 \\
\hline Rural other principal arterials & $7,347,686$ & $525,205.3$ & $95,287.8$ & 13.0 \\
\hline Total & $27,727,023$ & $7,484,360$ & 440,039 & 15.9 \\
\hline
\end{tabular}

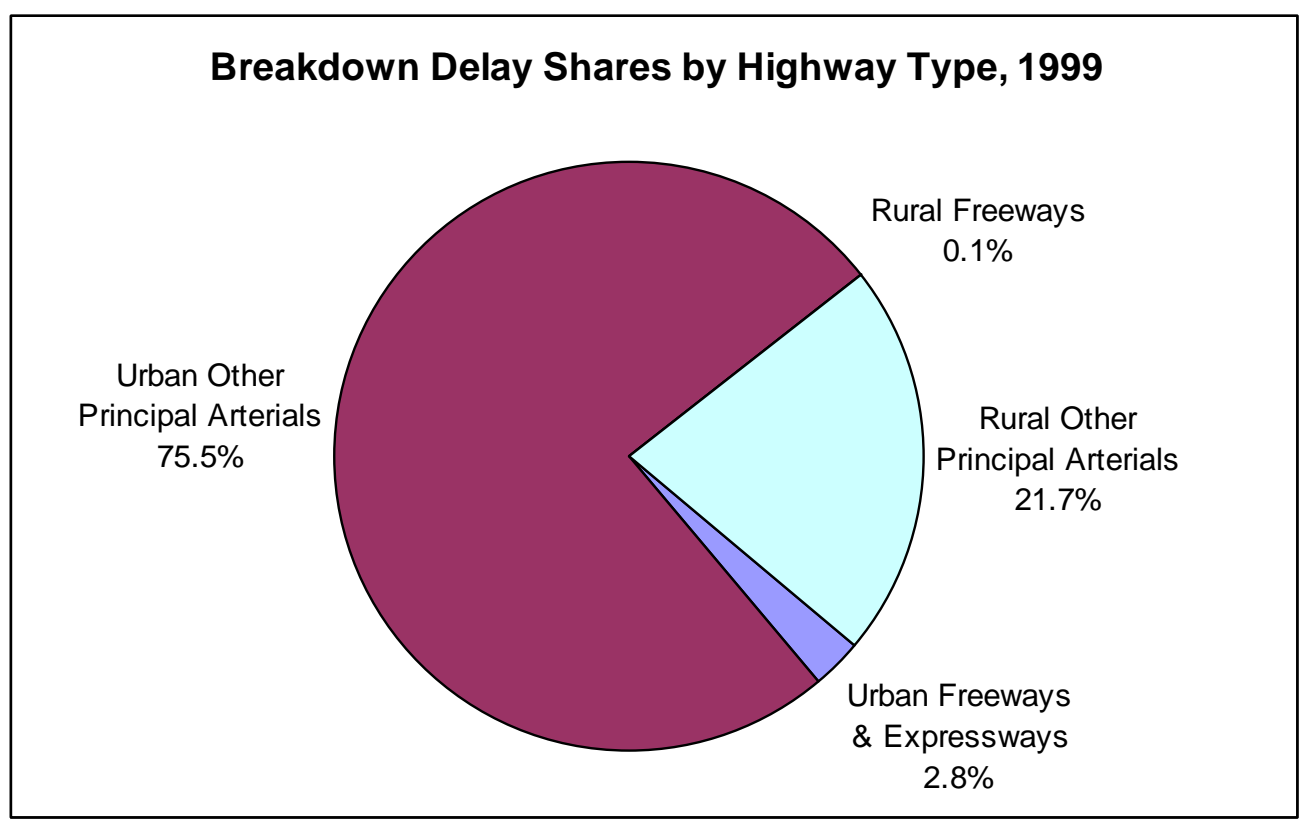

Fig. 12. TLC2 estimates that most delay from breakdowns is experienced on urban principal arterials and rural principal arterials. 
Table 17. Capacity reduction \& delay from breakdowns on freeways \& principal arterials, 1999

\begin{tabular}{|c|c|c|c|c|c|c|}
\hline Highway type & $\begin{array}{c}\text { Urban area } \\
\text { size* }\end{array}$ & $\begin{array}{l}\text { Peak } \\
\text { period }^{\dagger}\end{array}$ & $\begin{array}{c}\text { Congestion } \\
\text { level }^{\ddagger}\end{array}$ & $\begin{array}{c}\text { No. of } \\
\text { breakdowns }\end{array}$ & $\begin{array}{c}\text { Capacity } \\
\text { reduction } \\
(1,000 \text { vehs })\end{array}$ & $\begin{array}{c}\text { Delay } \\
(1,000 \text { veh-hrs })\end{array}$ \\
\hline \multirow{13}{*}{$\begin{array}{l}\text { Urban } \\
\text { freeways and } \\
\text { expressways }\end{array}$} & \multirow[t]{3}{*}{ Very large } & \multirow[t]{2}{*}{ Peak } & Congested & 161,342 & $151,723.4$ & $1,049.5$ \\
\hline & & & Not congested & 338,967 & $312,157.2$ & $1,429.3$ \\
\hline & & \multicolumn{2}{|l|}{ Off-peak } & $1,116,078$ & $1,044,797.7$ & $4,499.8$ \\
\hline & \multirow[t]{3}{*}{ Large } & \multirow[t]{2}{*}{ Peak } & Congested & 110,845 & $96,942.1$ & 208.8 \\
\hline & & & Not congested & 344,454 & $301,133.1$ & 894.1 \\
\hline & & \multicolumn{2}{|l|}{ Off-peak } & $1,021,315$ & $903,586.5$ & $2,278.6$ \\
\hline & \multirow[t]{3}{*}{ Medium } & \multirow[t]{2}{*}{ Peak } & Congested & 25,737 & $21,305.7$ & 45.8 \\
\hline & & & Not congested & 131,190 & $107,014.0$ & 86.4 \\
\hline & & \multicolumn{2}{|l|}{ Off-peak } & 353,838 & $294,319.7$ & 351.3 \\
\hline & \multirow[t]{3}{*}{ Small } & \multirow[t]{2}{*}{ Peak } & Congested & 33,753 & $26,703.6$ & 40.2 \\
\hline & & & Not congested & 344,573 & $273,062.2$ & 192.2 \\
\hline & & \multicolumn{2}{|l|}{ Off-peak } & 844,156 & $677,121.1$ & $1,054.0$ \\
\hline & \multicolumn{3}{|l|}{ Total } & $4,826,247$ & $4,209,866.3$ & $12,130.0$ \\
\hline \multirow{13}{*}{$\begin{array}{l}\text { Urban } \\
\text { other } \\
\text { principal } \\
\text { arterials }\end{array}$} & \multirow[t]{3}{*}{ Very large } & \multirow[t]{2}{*}{ Peak } & Congested & 175,462 & $18,223.6$ & $16,112.5$ \\
\hline & & & Not congested & 606,652 & $62,705.8$ & $42,915.6$ \\
\hline & & \multicolumn{2}{|l|}{ Off-peak } & $1,739,363$ & $188,400.5$ & $89,336.1$ \\
\hline & \multirow[t]{3}{*}{ Large } & \multirow[t]{2}{*}{ Peak } & Congested & 88,081 & $9,070.0$ & $4,050.8$ \\
\hline & & & Not congested & 503,611 & $51,569.7$ & $30,959.6$ \\
\hline & & \multicolumn{2}{|c|}{ Off-peak } & $1,334,201$ & $143,145.8$ & $53,210.0$ \\
\hline & \multirow[t]{3}{*}{ Medium } & \multirow[t]{2}{*}{ Peak } & Congested & 32,638 & $3,194.4$ & $1,119.2$ \\
\hline & & & Not congested & 213,015 & $21,915.7$ & $7,292.3$ \\
\hline & & \multicolumn{2}{|c|}{ Off-peak } & 555,408 & $59,920.6$ & $15,609.9$ \\
\hline & \multirow[t]{3}{*}{ Small } & \multirow[t]{2}{*}{ Peak } & Congested & 100,322 & $9,794.3$ & $3,290.1$ \\
\hline & & & Not congested & $1,008,981$ & $99,299.3$ & $24,462.8$ \\
\hline & & \multicolumn{2}{|l|}{ Off-peak } & $2,459,261$ & $256,766.9$ & $43,833.9$ \\
\hline & \multicolumn{3}{|l|}{ Total } & $8,816,994$ & $924,006.6$ & $332,192.7$ \\
\hline Rural & & Peak & Congested & 17,255 & $14,126.9$ & 7.4 \\
\hline freeways & & & Not congested & 620,928 & $497,693.1$ & 124.8 \\
\hline & & Off-peak & & $1,622,264$ & $1,313,462.2$ & 296.1 \\
\hline & & Total & & $2,260,447$ & $1,825,282.2$ & 428.2 \\
\hline Rural other & & Peak & Congested & 53,278 & $4,803.8$ & $4,232.1$ \\
\hline principal & & & Not congested & $1,502,419$ & $138,562.7$ & $25,176.8$ \\
\hline arterials & & Off-peak & & $3,891,863$ & $381,838.8$ & $65,878.9$ \\
\hline & & Total & & $5,447,560$ & $525,205.3$ & $95,287.8$ \\
\hline Total & & & & $21,351,248$ & $7,484,360.4$ & $440,038.8$ \\
\hline
\end{tabular}

* Urban area size categories are based on population: very large - more than 3 million; large - 1 to 3 million; medium 0.5 to 1 million; small - less than 0.5 million.

${ }^{\dagger}$ Peak periods: 6:00 am to 9:30 am and 3:30 pm to 7:00 pm Monday through Friday; all others considered non-peak.

$¥$ A roadway section is considered congested during the peak periods if its Volume/Service Flow Ratio $(\mathrm{V} / \mathrm{SF})$ is greater than $95 \%$. 


\subsection{RELIABILITY}

\subsubsection{Methodology}

The methodology for estimating the number of breakdowns is suspect. Breakdowns were estimated to be proportional to crashes. However, no direct relationship between crashes and breakdowns has been established - though both may be related to VMT. This method is accorded a low level of confidence.

The Monte Carlo simulation method used to assign breakdowns to a specific facility (freeway or arterial) should be reasonable since it can be argued that breakdowns are predominantly random, with a probability of a breakdown occurring on a link related to the link's VMT.

The TLC study used standard, well-established procedures and methods derived from traffic flow theory to estimate delay (TRB, Highway Capacity Manual 1985). However, the confidence levels of the methodologies used to determine capacity losses and delays due to non-breakdowns depend on the type of facility where the breakdown occurred. For freeways, both methodologies can be classified as having a high degree of confidence. However, due to the number of assumptions required to estimate capacity loss and delay on principal arterials, both methodologies can be qualified as having a medium degree of confidence.

It should be noted that due to the low level of confidence for the data and methods used to estimate the total number of breakdowns, the delay estimates produced by TLC 2 are accorded a low level of confidence.

\subsubsection{Data \& Key Assumptions}

Number of Breakdowns: The number of breakdowns relative to crashes was assumed based on a few published reports that have compiled limited data on crashes and breakdowns on freeways. TLC2 assumes there are eight times as many breakdowns as crashes, but the level of confidence for this assumption is fairly low. Even if this assumption for freeways is reasonable, it is unclear whether ratios based on freeway crashes would be equally valid for principal arterials.

Number of Lanes: The number of lanes available under normal conditions was taken from the HPMS data set. This data is considered reliable for all highway types.

Lane Blockage: Lane block probabilities were based on statistics in the literature and from data provided by a few highway service patrols (mostly freeway data), as well as assumptions based on engineering judgment. These probabilities and assumptions are considered of medium confidence for freeways and of lower confidence for principal arterials.

Breakdown Duration: Assumptions regarding breakdown duration were based on very limited studies conducted on freeways. Their application to other freeways is only of moderate confidence, and their applicability to arterials is of low confidence.

Traffic Demand \& Surface Street Characteristics: The same traffic demand data and surface street assumptions used to estimate crash delays were used for breakdowns. Therefore, the same caveats apply: traffic demand data is accorded a low level of confidence and surface street characteristics are accorded a medium level of confidence (see section 3.3.2). 


\section{WORK ZONES}

\subsection{METHODOLOGY}

The method used to estimate delays due to work zones was similar to the one used for crashes since both types of events produce localized bottlenecks. The relevant variables defining a work zone are its location (urban or rural), the total number of lanes, the number of lanes closed, the length of the work zone, and the duration of the work. Since 1999 data was not available, the work zone estimates in the study are for May 2001 through May 2002.

The basic process for estimating the delay from work zones includes the following:

$\underline{\text { Step } 1 .}$ An inventory of work zones and their attributes was obtained from the Rand McNally website.

Step 2. Capacity loss was estimated based on the number of lanes normally open and the number of lanes closed due to the work zone, along with the length of time the lanes were closed.

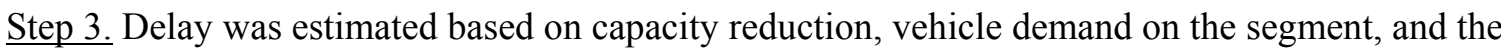
duration of the work zone.

These steps are described in more detail in the paragraphs below.

\subsubsection{Identifying Work Zones}

Identifying the location and time span of work zones was problematic. There are currently two nationallevel data sets that identify work zones: the Rand McNally website and FHWA's Fiscal Management Information System (FMIS). However, each has significant limitations.

\section{Rand McNally Construction Information Data}

Rand McNally Construction Information for North America is an Internet-based searchable highway construction information system maintained to inform drivers of work zone activities. It allows the users to find highway construction information by road type, by signed route, by beginning and ending dates, and by state. It includes location, beginning and ending dates, and, in some cases, lane closures for existing and scheduled work zones. However, any data regarding work zones that are no longer active are purged from their database. In addition, data on scheduled work zones are typically only accurate to about four months into the future from the time it is accessed. Finally, this data is collected from state and local agencies by a team of Rand McNally employees. Thus, time and resource considerations can affect the amount of data that is gathered. ORNL downloaded work zone data for May 2001 through May 2002 and used it as a surrogate for 1999. Statistics for the number of work zone miles and mile-days by highway and area type are given in Table 18.

\section{Fiscal Management Information System (FMIS)}

The FMIS database, maintained by FHWA, tracks the allocation of funding obligated to states for federally funded highway projects. According to FHWA, the database includes information on highway and bridge construction and maintenance projects, their locations, the type of activity funded, and other data. The FMIS data for calendar year 1999 was acquired and evaluated as an alternate or complimentary source of information on work zone characteristics. Initial review of the data suggested that it might be 
useful for developing an inventory of work zones and their characteristics. However, further examination showed that FMIS's project time and location characteristics did not provide enough detail to estimate capacity reduction accurately. Therefore, it was decided that the Rand McNally database was more appropriate for the TLC and TLC2 studies.

Table 18. Work zone length by highway and area type, May 2001 to May 2002

\begin{tabular}{llrr}
\hline & & \multicolumn{2}{c}{ Work zone length } \\
Highway type & Urban area size & Miles & \multicolumn{1}{c}{ Mile-days } \\
\hline Urban freeways and & Very large & 165.6 & $54,053.8$ \\
expressways & Large & 290.1 & $74,409.8$ \\
& Medium & 139.2 & $30,523.7$ \\
& Small & 318.3 & $85,731.7$ \\
& Total & 913.2 & $244,719.0$ \\
\hline Urban other principal & Very large & 36.8 & $11,130.7$ \\
arterials & Large & 36.9 & $5,808.8$ \\
& Medium & 21.8 & $5,646.0$ \\
& Small & 555.6 & $125,730.4$ \\
& Total & 651.1 & $148,315.9$ \\
\hline Rural freeways & & $1,319.3$ & $328,207.3$ \\
\hline Rural other principal arterials & & $1,327.3$ & $283,296.7$ \\
\hline Total & & $4,210.9$ & $1,004,538.9$ \\
\hline
\end{tabular}

\subsubsection{Estimating Loss of Capacity}

Work zones produce a loss of capacity that depends on the total number of lanes normally available and the number of lanes closed. The "end-of-transition" and "activity-area" capacities of work zones' open lanes as a function of the available lanes and the environment (rural or urban) of the facility were determined using data from the Highway Capacity Manual, Special Report 209 (TRB 1985) and studies performed by Dixon (1996). Table 19 shows the end-of-transition and activity-area capacities of work zones' open lanes as a function of the available lanes and the environment (rural or urban) of the facility. The table was obtained by combining information from Tables 4 and 5 . 
Table 19. Capacities of open lanes at work zones

\begin{tabular}{cclcc}
\hline \multicolumn{2}{c}{ Number of lanes } & Rural or & \multicolumn{2}{c}{ Capacity (vehicles per hour per lane) } \\
\cline { 1 - 2 } \cline { 5 - 5 } Normal & Closed & urban & End of transition & Activity area \\
\hline 2 & 1 & Rural & 1,300 & 1,210 \\
2 & 2 & Rural & $1,300^{*}$ & $1,210^{*}$ \\
2 & 1 & Urban & 1,690 & 1,515 \\
3 & 1 & Rural & 1,490 & 1,490 \\
3 & 2 & Rural & 1,170 & 1,170 \\
3 & 1 & Urban & 1,490 & 1,490 \\
3 & 2 & Urban & 1,640 & 1,440 \\
4 & 1 & Urban & 1,520 & 1,520 \\
4 & 2 & Urban & 1,480 & 1,480 \\
4 & 3 & Urban & 1,170 & 1,170 \\
5 & 1 & Urban & 1,520 & 1,520 \\
5 & 2 & Urban & 1,480 & 1,480 \\
5 & 3 & Urban & 1,370 & 1,370 \\
5 & 4 & Urban & 1,170 & 1,170 \\
\hline
\end{tabular}

* Crossover work zone, both ways operate with one lane. This case has not been considered in this study

Information from Table 5 was given priority in creating Table 16 above. Where information was missing, data provided in Table 4 was used. For some combinations (e.g., 5 available lanes and 3 lanes closed), the closest data from Table 16 that provided the same number of open lanes was used. For the previous example, 1,480 vphpl would be used, which corresponds to 4 lanes available and 2 open lanes. When the information was derived from Table 4 , the same values were adopted for the end-of-transition and activity-area capacities.

To determine the capacity of open lanes in a work zone, it was necessary to know the total number of available lanes and the number of lanes closed. The total number of lanes on each highway segment being analyzed was obtained from HPMS. If the work zone database contained the number of lanes closed, that information was used. For cases where this data was not available, data from the Highway Capacity Manual, Special Report 209 (TRB 1985) was used to estimate the number of lanes closed due to the construction/maintenance work (Table 20). 
Table 20. Probability distribution of the number of lanes closed in work zone areas

\begin{tabular}{llccc}
\hline $\begin{array}{l}\text { Number of } \\
\text { available lanes }\end{array}$ & Type of work & $\begin{array}{c}\text { 1 Lane } \\
\text { closed }\end{array}$ & $\begin{array}{c}\text { 2 Lanes } \\
\text { closed }\end{array}$ & $\begin{array}{c}\text { 3+ Lanes } \\
\text { closed }\end{array}$ \\
\hline 2 Lanes & New construction or road widening & 1.000 & 0.000 & 0.000 \\
& Other type of work & 1.000 & 0.000 & 0.000 \\
3 Lanes & New construction or road widening & 1.000 & 0.000 & 0.000 \\
& Other type of work & 0.000 & 1.000 & 0.000 \\
More than 3 lanes & New construction or road widening & 1.000 & 0.000 & 0.000 \\
& Other type of work & 0.000 & 1.000 & 0.000 \\
\hline
\end{tabular}

Finally, to compute the travel time delays at work zones, it was necessary to know the physical length of the work zone. This information was obtained from the Rand McNally database, where available. If this data was missing, a work zone length of 0.5 miles was assumed for bridgework, or a length of 1.0 mile was assumed for other work.

\subsubsection{Delays Due to Work Zones}

Work zone delay was estimated using a method similar to the one used for crashes, since both events produce localized bottlenecks. Delay estimates were based on (1) the capacity of the open lanes at the work zone (determined based on Table 19), (2) the traffic demand as a function of time of day and day of week, and (3) and the location of the work zone (determined using the same procedure as for crashes). Delays were computed for each hour of each day during the entire duration of the work (using the corresponding demand) and then added to determine the total delays due to the work zone.

To estimate the queues that would form upstream of the work zones and to calculate the delays produced, the "end-of-transition" capacities were used (see Table 19), while the "activity area" capacities were used to compute the travel time delays. One difference, however, between crashes and work zones is that travelers can often adjust their travel behavior (e.g., trip re-routing, trip re-scheduling, and trip canceling) during the duration of the construction period. When this is a feasible alternative to drivers, the demand will be reduced. This effect was not considered in the present study.

\subsection{RESULTS}

Capacity losses due to 1,063 work zones reported by Rand McNally to be active on freeways and principal arterials during May 2001 to May 2002 amounted to an estimated 8.4 billion vehicles per year (Table 21). Resulting delay is estimated at 889 million vehicle-hours. The study estimates that most of the delay was experienced on urban freeways ( 83 percent) and rural freeways (15 percent). Work zones on urban and rural principal arterials combined are estimated to only account for about 2 percent of delay. However, delay for these highways may be underestimated, since work zone data for freeways is likely to be more complete.

The reliability of capacity loss and delay estimates for work zones is unclear due to potential data gaps in the Rand McNally database (the Rand McNally database was not designed for this application). Small or short-term construction or utility work zones may not be included, and the database is less accurate at identifying work zones that will begin activity four months into the future. In addition, work zone impacts are complicated to measure since drivers often have prior knowledge of work zones and can reroute, reschedule, or cancel trips accordingly. This is especially true for long-term work zones. However, our estimates are based on the assumption that no rerouting, rescheduling or reduced mobility occurred. 


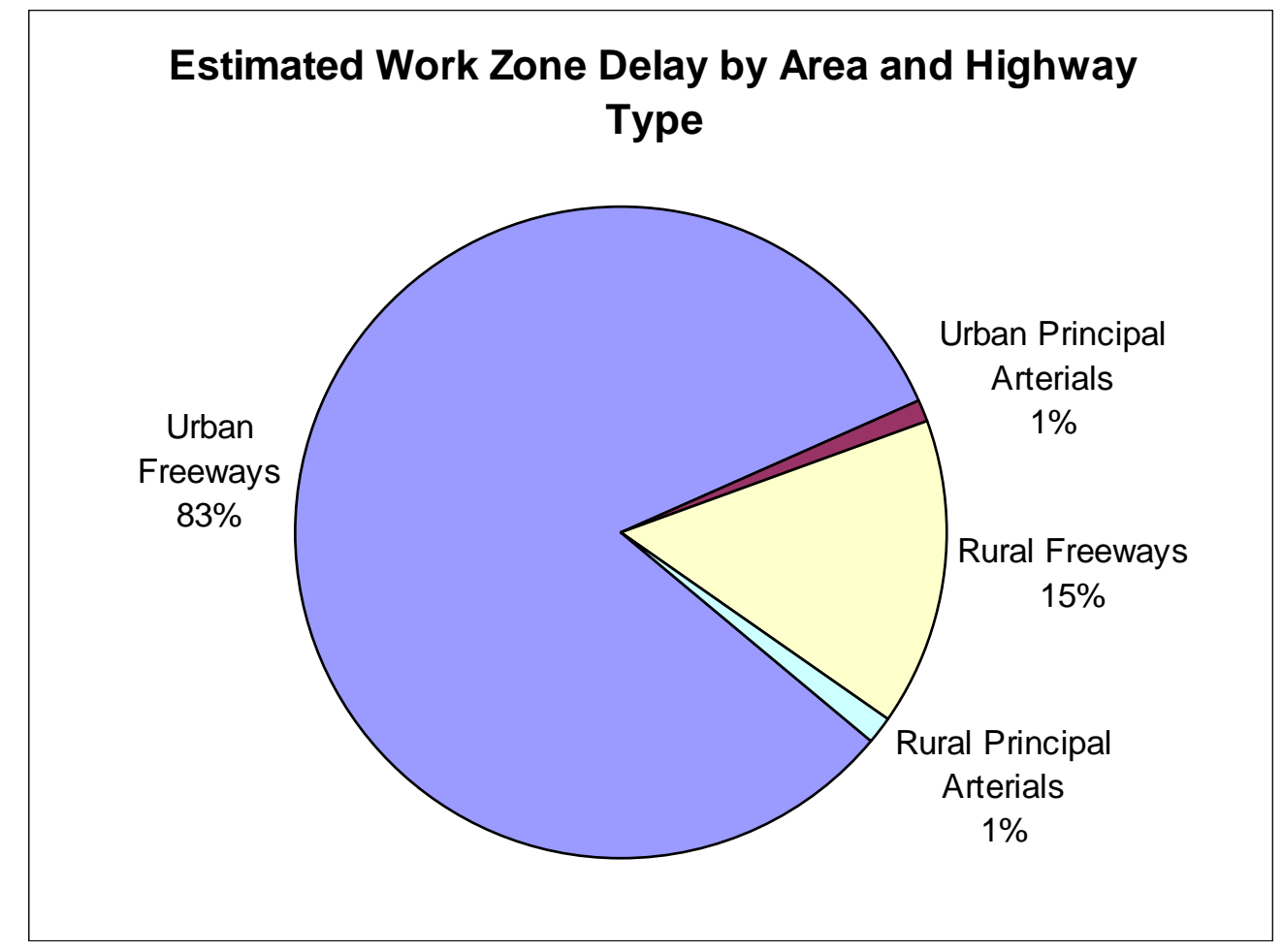

Fig. 13. TLC2 estimates that 98 percent of delay from work zones was experienced on freeways. 
Table 21. Capacity reductions \& delay due to work zones

\begin{tabular}{|c|c|c|c|c|c|}
\hline Highway type & $\begin{array}{c}\text { Urban } \\
\text { area size* }\end{array}$ & $\begin{array}{c}\text { Peak } \\
\text { period }^{\dagger}\end{array}$ & $\begin{array}{c}\text { Congestion } \\
\text { level }^{\ddagger}\end{array}$ & $\begin{array}{c}\text { Capacity } \\
\text { reduction } \\
(1,000 \text { vehs })\end{array}$ & $\begin{array}{c}\text { Delay } \\
(1,000 \text { veh-hrs }) \\
\end{array}$ \\
\hline \multirow{13}{*}{$\begin{array}{l}\text { Urban freeways } \\
\text { and expressways }\end{array}$} & \multirow{3}{*}{$\begin{array}{l}\text { Very } \\
\text { large }\end{array}$} & \multirow{2}{*}{ Peak } & Congested & $11,509.1$ & $19,443.3$ \\
\hline & & & Not congested & $32,465.9$ & $41,708.0$ \\
\hline & & \multicolumn{2}{|l|}{ Off-peak } & $167,104.8$ & $105,716.0$ \\
\hline & \multirow{3}{*}{ Large } & \multirow{2}{*}{ Peak } & Congested & $20,568.9$ & $45,749.2$ \\
\hline & & & Not congested & $86,742.3$ & $61,593.1$ \\
\hline & & \multicolumn{2}{|l|}{ Off-peak } & $407,782.2$ & $174,944.0$ \\
\hline & \multirow{3}{*}{ Medium } & \multirow{2}{*}{ Peak } & Congested & $5,980.1$ & $9,412.3$ \\
\hline & & & Not congested & $43,528.8$ & $37,576.7$ \\
\hline & & \multicolumn{2}{|l|}{ Off-peak } & $188,133.4$ & $59,126.2$ \\
\hline & \multirow{3}{*}{ Small } & \multirow{2}{*}{ Peak } & Congested & $6,178.9$ & $5,485.2$ \\
\hline & & & Not congested & $147,668.6$ & $61,216.2$ \\
\hline & & \multicolumn{2}{|l|}{ Off-peak } & $584,620.5$ & $108,267.5$ \\
\hline & \multicolumn{3}{|l|}{ Total } & $1,702,283.3$ & $730,237.8$ \\
\hline \multirow{13}{*}{$\begin{array}{l}\text { Urban other } \\
\text { principal arterials }\end{array}$} & \multirow{3}{*}{$\begin{array}{l}\text { Very } \\
\text { large }\end{array}$} & \multirow{2}{*}{ Peak } & Congested & $4,258.3$ & 40.2 \\
\hline & & & Not congested & $34,905.5$ & $1,619.2$ \\
\hline & & \multicolumn{2}{|l|}{ Off-peak } & $148,822.3$ & 782.2 \\
\hline & \multirow{3}{*}{ Large } & \multirow{2}{*}{ Peak } & Congested & $3,928.5$ & 76.6 \\
\hline & & & Not congested & $6,368.1$ & 50.6 \\
\hline & & \multicolumn{2}{|l|}{ Off-peak } & $39,126.9$ & 174.0 \\
\hline & \multirow{3}{*}{ Medium } & \multirow{2}{*}{ Peak } & Congested & $1,770.3$ & 4.9 \\
\hline & & & Not congested & $9,986.2$ & 90.8 \\
\hline & & \multicolumn{2}{|l|}{ Off-peak } & $44,674.3$ & 130.1 \\
\hline & \multirow{3}{*}{ Small } & \multirow{2}{*}{ Peak } & Congested & $7,949.2$ & 53.3 \\
\hline & & & Not congested & $207,754.7$ & $3,564.4$ \\
\hline & & \multicolumn{2}{|l|}{ Off-peak } & $819,674.4$ & $3,163.5$ \\
\hline & \multicolumn{3}{|l|}{ Total } & $1,329,218.4$ & $9,749.9$ \\
\hline & & Peok $>$ & Congested & $29,293.2$ & $18,537.2$ \\
\hline Rural freewavs & & Peak & Not congested & $511,286.9$ & $31,485.9$ \\
\hline Kural Ireeways & & Off-peak & & $2,054,204.2$ & $86,474.3$ \\
\hline & & Total & & $2,594,784.2$ & $136,497.3$ \\
\hline & & Peok $>$ ( & Congested & $9,361.5$ & 133.5 \\
\hline Rural other princir & al arterials & Peak & Not congested & $558,416.9$ & $4,686.4$ \\
\hline Rural other princip & al arterials & Off-peak & & $2,157,557.9$ & $7,722.9$ \\
\hline & & Total & & $2,725,336.3$ & $12,542.8$ \\
\hline Total & & & & $8,351,622.2$ & $889,027.7$ \\
\hline
\end{tabular}

* Urban area size categories are based on population: very large - more than 3 million; large - 1 to 3 million; medium 0.5 to 1 million; small - less than 0.5 million.

${ }^{\dagger}$ Peak periods: 6:00 am to 9:30 am and 3:30 pm to 7:00 pm Monday through Friday; all others considered non-peak.

* A roadway section is considered congested during the peak periods if its Volume/Service Flow Ratio (V/SF) is greater than $95 \%$. 


\subsection{RELIABILITY}

\subsubsection{Methodology}

The confidence level of the methodology used to determine capacity losses depends on the type of facility where the work zone was located. The data used to estimate capacity loss is based on only two studies of freeway work zone impacts, and these studies stress the high variability that exist from site to site in terms of lane capacities. Therefore, higher confidence is given to freeway work zones than for work zones located on arterials.

The methodology used to estimate delay for TLC2 is based on traffic flow theory and is well established in the transportation community (TRB, Highway Capacity Manual 1985). This methodology can be qualified as having a high degree of confidence for freeway work zones, and a medium level for arterials and other facilities with unrestricted access. The reliability of the delay estimates, however, is also affected by the reliability of the data and methods used to determine capacity loss and demand.

\subsubsection{Data \& Key Assumptions}

Work Zone Inventory \& Characteristics: The Rand McNally dataset used to identify and locate work zones is accorded a medium to low level of reliability for the application for which it was used. The primary short-comings include (1) the fact that May 2001 to May 2002 had to be used as a surrogate for 1999 data and (2) the likelihood that smaller, short-term work zones and work activities beginning more than four months into the future are not included in the data.

Traffic Demand \& Surface Street Characteristics: The same traffic demand data and surface street assumptions used to estimate crash delays were used for breakdowns. Therefore, the same caveats apply: traffic demand data is accorded a low level of confidence and surface street characteristics are accorded a medium level of confidence (see section 3.3.2). 


\section{WEATHER}

Adverse weather conditions have a major impact on the operation of our Nation's roads. Weather such as fog, rain, snow, and ice can reduce visibility and/or vehicle traction, causing drivers to reduce speeds and increase following distances. This reduces roadway capacity and increases delays. It can also cause drivers to cancel or re-schedule trips or re-route them around problem areas (e.g., roads prone to localized flooding or steep grades that become slick in icy weather), but these effects were outside the scope of this study. These events can also cause crashes or disable vehicles, further reducing capacity and increasing delays. However, it should be noted that the study did not attempt to determine crashes or disabled vehicles caused by these weather events nor did it attempt to consider the additive impacts of these events taking place during weather events - for example, it did not attempt to determine the additive delay caused from a crash occurring in a snowstorm. Finally, rain, fog, snow, and ice are not the only weather events that can cause temporary delay. Solar glare, dust storms, gusting winds, flash floods, melting and re-freezing of snow and ice, and forest fires, as well as catastrophic events such as floods and hurricanes, can all reduce capacity and cause delay. However, due to data limitations and time and funding constraints, these events were not included in the study. Therefore, the weather-related delay estimates should be viewed in the context of these limitations in scope.

\subsection{METHODOLOGY}

Capacity reductions and delays from fog, snow, and ice were estimated in the initial phase of the TLC study. For TLC2, a revised methodology was used to estimate capacity reduction and delays for these weather phenomena, along with those resulting from rain. The primary difference in the methodologies was in the capacity reductions attributed to each type of weather event. In the initial TLC study, capacity reductions were based on estimates reported in a limited number of studies in the literature. However, for TLC2, newly available capacity and speed reduction estimates from FHWA's Road Weather Management Program were used.

The process for estimating delay for adverse weather consisted of the following tasks:

Step 1. Identify all rain-, fog-, snow-, and ice-related weather events, along with the temporal and location characteristics of each event. Location was defined in terms of public forecast zones or "region of influence" defined by weather stations. Archived weather databases compiled by the National Climatic Data Center (NCDC) were used to identify and locate events.

Step 2. Map the weather events to the highway network system. Some weather-related data follows county boundaries. This is convenient since highway data from HPMS is available by county. However, for weather data that is not county-based, a GIS-based computer model was used to map the weather data to the National Highway Performance Network (NHPN).

$\underline{\text { Step 3 }}$. Estimate capacity losses on highways in impacted counties. Capacity losses due to weather were based on weather-induced capacity and speed reductions estimated by FHWA's Road Weather Management Program.

Step 4. Estimate the normal delay (congestion delay without adverse weather condition) for roadways in each county during the time of the event.

Step 5. Estimate the delay with adverse weather conditions for roadways in each county during the time of the event. This was accomplished by repeating the same delay estimation step with 
the appropriate capacity reduction for the weather type. For each link, if the average speed without the weather event was greater than the typical travel speed for that event, the delay was calculated based on the difference between those two speeds. Otherwise, the delay was not adjusted.

Step 6. Calculate the delay induced by adverse weather conditions for each county as the difference in the estimated delay with the adverse weather condition and the estimated delay without the adverse weather condition.

Step 7. Calculate the delay induced by adverse weather conditions for each public forecast zone or "region of influence" defined by weather stations as the sum of the delay for all involved counties multiplied by the percentage of each county's major arterial mileage lying within the public forecast zone.

\subsubsection{Identifying Adverse Weather Conditions}

The first step in assessing the effect of adverse weather conditions on travel was to identify events with the potential to reduce capacities and cause delays. Adverse weather events were identified using archived weather databases from the National Climatic Data Center (NCDC). The Center has long served the Nation as a national resource for climate information.

NCDC's "Storm Data and Unusual Weather Phenomena" database was used to identify fog- and icerelated weather events. The Storm Data database contains a chronological listing, by state, of events such as hurricanes, tornadoes, thunderstorms, hail, floods, drought conditions, lightning, high winds, snow, temperature extremes, and other weather phenomena. Since the database identifies events in more-specific terms than used in the study, each fog- and ice-related event was assigned to more broad categories as shown in the table below.

Table 22. NWS weather event categories used in the study

\begin{tabular}{l|ll}
\hline Fog-related events & Ice-related events & \\
\hline Fog & Freezing Fog & Ice Storm \\
& Freezing Rain & Icy Roads \\
& Freezing & Light Freezing Rain \\
& Rain/Sleet & Light Snow/Freezing \\
& Glaze & Precipitation \\
& Ice & Snow \& Ice \\
& Ice Roads & \\
\hline
\end{tabular}

In principle, the database only includes storms and other important climatological events having sufficient intensity to cause injuries, loss of life, and/or significant property damage. "Significant" events, for the purposes of inclusion in the database, include those causing losses of at least $\$ 1,000$. Within the National Weather Service Weather Services Operation Manual (Chapter F-42, Storm Data and Related Reports), quantitative measures and procedures are provided to identify severe and/or significant weather and weather-related events. However, much of the data is compiled by NWS personnel based on their judgment in ascertaining the severity of a meteorological event. Furthermore, it is possible for some events, especially fog, to have a significant impact on traffic without causing enough financial damage to be classified as severe. 
In the database, the location and impacted area for an event is described in terms of (1) the county name and nearby town, or by mileage and direction from one town or between two towns, or (2) public forecast zones. Public forecast zones are geographical areas within a state designated by the Weather Service Forecast Offices (WSFO) and Next Generation Radar (NEXRAD) Weather Service Forecast Offices (NWSFO) with consent of the Regional Headquarters. Ideally, a forecast zone is an area with sufficient climatological and meteorological homogeneity to allow a single forecast to serve as the local forecast for the communities within the area. Each WSFO's area of responsibility is divided into forecast zones. The only exceptions are a few mountainous or largely unpopulated areas of the far western United States, certain wilderness areas in Alaska, island areas of the Pacific Region, and Isle Royale in Lake Superior.

Zone boundaries are typically determined by considering:

- Homogeneity of climate and usual weather features

- Population distribution

- Local communications and dissemination capabilities

In practice, most public forecast zones follow county boundaries, although some counties might be split into multiple public forecast zones and some forecast zones might consist of multiple counties. For example, public forecast zone boundaries in Alabama typically coincide with county boundaries. This is true for all counties except Baldwin and Mobile counties, which border the Gulf of Mexico. The local WSFO further divides these two counties into four public forecast zones. However, in some states, public forecast zone boundaries do not follow county boundaries. For example, public forecast zones in Utah and Colorado typically span several counties. Each public forecast zone is assigned a Universal Generic Code. This allows local offices to create their own "regional" areas.

\section{Rain and Snow}

Rain- and snow-related weather events were identified using the National Weather Service's "Hourly Precipitation Data (HPD) TD3240" and "Cooperative Summary of Day TD3200" databases. The HPD database contains hourly precipitation amounts recorded by rain gages located at National Weather Service, Federal Aviation Administration, and cooperative observer stations. HPD includes maximum precipitation for nine (9) daily periods, ranging in length from 15 minutes to 24 hours, for selected stations.

Unfortunately, the HPD only contains precipitation information; it has little information on the type of precipitation, such as rain, snow, or freezing rain. Therefore, the Summary of Day database was used to infer the precipitation type. The Summary of Day database includes daily characteristics such as maximum/minimum temperatures, precipitation, and snowfall/snow depth. Some stations have additional data such as evaporation, soil temperature, peak wind gust, etc. This data is a compilation of daily observations initially obtained from state universities, state cooperatives, and the National Weather Service. Most stations that collect HPD data also collect Summary of Day data. However, for the small percentage that did not collect this data during the study year, data from the nearest station that did collect it was used.

A few assumptions were made to infer precipitation type. If the Summary of Day data gives an amount in the "snow/ice pellets" field, it was assumed that all of the precipitation for that day was snow. The precipitation amount in the HPD was also assumed to be snow if the maximum temperature as given in the Summary of Day data was $32^{\circ} \mathrm{F}$ or less. Another limitation of the data is the accuracy of precipitation gauges. These gauges measure snow and ice somewhat less accurately than rain. 
The HPD and Summary of Day databases contain information on the locations of the cooperative weather stations (i.e., the point locations where the weather measurements are made). However, the weather data for these one-dimensional points must be applied to two-dimensional areas. This was accomplished by creating Thiessen Polygons around the weather station location points. Thiessen polygons - also referred to as the Dirichlet Tessellation or the Voronoi Diagram - define the individual "region of influence" around each point within a set of points. Thiessen polygons are polygons whose boundaries define the area that is closest to each point relative to all other points. Thiessen polygons, which are generated from a set of points, are mathematically defined by the perpendicular bisectors of the lines between all points.

By using the Thiessen polygons, this study assumes the adverse weather condition is represented by the weather data collected at the cooperative weather station at the centroid and that the weather is the same throughout the polygon. This assumption is adequate if a sufficient number of cooperative weather stations are available and the polygons are small enough to describe weather phenomenon effectively. Some of the resulting polygons in the TLC2 study are somewhat large due to the sparsity of weather stations in some areas. However, these are typically lower population areas that are likely to generate less traffic. Therefore, any inaccuracies in weather impacts within these larger polygons should not greatly affect delay estimates. The figure below shows the Thiessen polygons used with this data set.

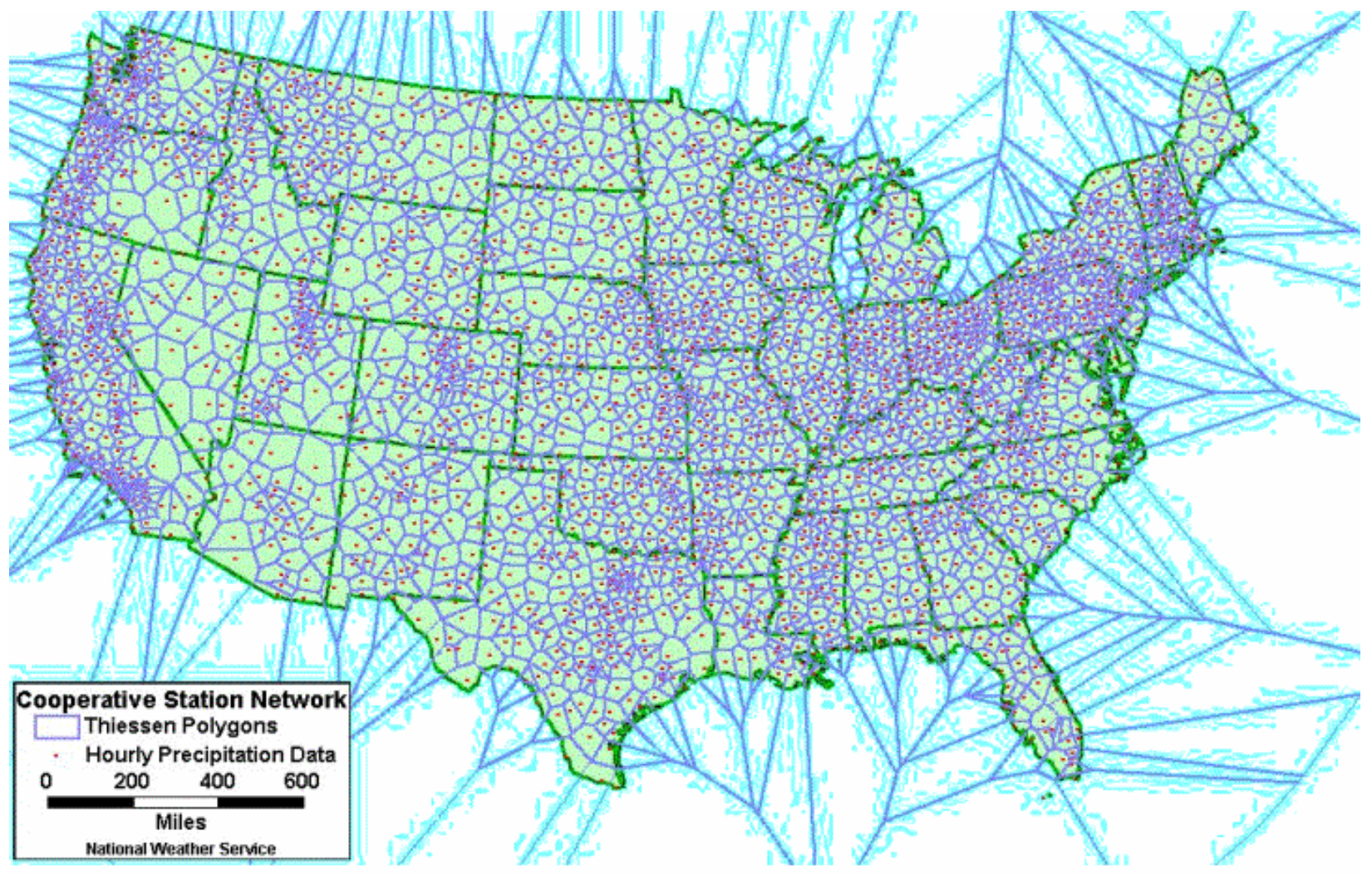

Fig. 14. Weather areas defined in terms of Thiessen polygons

\subsubsection{Mapping Adverse Weather Conditions to Highway Segments}

FHWA's HPMS was used to help estimate weather impacts in terms of traffic capacity losses and delays. HPMS provides traffic operation information by highway functional class, county, and state. Since the locations of fog- and ice-related weather events are described using public forecast zones and rain- and snow-related events are assigned to Thiessen polygons, additional effort is needed to map these weather conditions to HPMS data, which is county-based. To calculate weather impacts, the percentage of each 
county's major arterial mileage affected within a given public forecast zone and/or Thiessen polygon was determined for each public forecast zone and Thiessen polygon.

Because the HPMS does not include coordinate data (i.e., longitude and latitude), an additional database was used to help map the coordinate-based weather data to highway segments. FHWA's National Highway Planning Network (NHPN) Ver. 2.2 was used to establish linkage between weather data and counties (U.S. DOT/FHWA 2000). The NHPN, which has been under development since the mid 1980s, was originally assimilated from a variety of sources at a nominal scale of 1:2 million and contains a set of data attributes that are suited to analytical modeling of large-scale transportation activities. The accuracy of the Version 2 database has changed from a scale of 1:2 million to a scale of 1:100,000 (an accuracy of about 80 meters rather than 1,500 ).

The method for mapping highway links to coordinate-based weather data consisted of the following steps:

1. "Cut" All NHPN Links at Public Forecast Zone and Thiessen Polygon Boundaries All NHPN links (roadway segments) are already "cut" at county boundaries. In order words, each NHPN link lies entirely within a county boundary. In order to establish linkage with coordinate-based weather data, all NHPN major arterial links were "cut" at public forecast zone and Thiessen polygon boundaries.

2. Aggregate All NHPN Major Arterial Links by Public Forecast Zone and Thiessen Polygon Aggregated mileages can be calculated for each public forecast zone and each Thiessen polygon based on the NHPN major arterial link's public forecast zone and Thiessen polygon subdivision.

3. Determine Each County's Percentage of Major Arterial Mileage Lying within Each Forecast Zone and Thiessen Polygon

Using the information obtained from the previous step, the percentage of each county's major arterial mileage within each public forecast zone and Thiessen polygon was determined.

\subsubsection{Estimating Capacity Losses}

Adverse weather conditions affect capacity and reduce operating speeds significantly, and each type of weather affects highway travel differently. The Transportation Research Board's Highway Capacity Manual discusses the traffic operations impacts of rain, snow, and fog and provides quantitative capacity and speed reduction factors based on limited studies - quantitative information on ice is not provided. For the initial study, information from the Highway Capacity Manual and studies by Lamm et al. (1990), Ibrahim and Hall (1994), Hogema et al. (1994), Aron et al. (1994), and Brilon and Ponzlet (1995) were used to produce estimates of reduced capacity. The revised study used capacity reduction factors compiled from various sources and studies by the Federal Highway Administration's (FHWA's) Road Weather Management Program.

The Road Weather Management Program was established by the FHWA Office of Operations to facilitate deployment of integrated road weather systems, decision support applications, and tools and practices in response to adverse weather that meet the needs of all transportation system users. Under this program, the Office of Operations has collected and compiled, from several sources, quantitative weather impacts on different roadway facilities at different locations. These impacts are summarized in the following table. 
Table 23. Mobility impacts of weather events*

\begin{tabular}{|c|c|}
\hline Highway type & Mobility impacts \\
\hline \multirow{4}{*}{ Urban freeways } & Light rain reduces speed by roughly $10 \%$, decreasing capacity by approximately $4 \%$. \\
\hline & Heavy rain decreases speed by about $16 \%$, lowering capacity by roughly $8 \%$. \\
\hline & Light snow reduces capacity by $5 \%$ to $10 \%$, depending upon accumulation. \\
\hline & $\begin{array}{l}\text { In heavy snow, speeds decline by nearly } 38 \% \text { suggesting a } 25-30 \% \text { reduction in } \\
\text { capacity. }\end{array}$ \\
\hline \multirow{4}{*}{ Rural freeways } & $\begin{array}{l}\text { When visibility is below } 530 \text { feet, speeds decline by } 13 \% \text {. } \\
\text { Capacity would be reduced by about } 6 \% \text {. }\end{array}$ \\
\hline & $\begin{array}{l}\text { Under heavy precipitation and wet pavement conditions, speeds decrease by } 26 \% \text {. } \\
\text { Capacity would drop by nearly } 10 \% \text {. }\end{array}$ \\
\hline & $\begin{array}{l}\text { Under severe conditions (i.e., visibility below } 0.23 \text { miles, wind speed over } 30 \mathrm{mph} \text {, } \\
\text { heavy snowfall and snow-covered pavement), speeds are reduced by } 41 \% \text {. }\end{array}$ \\
\hline & Capacity would decrease by roughly $14 \%$. \\
\hline \multirow{5}{*}{ Arterials } & Rain reduces speed by $10 \%$ and capacity by $6 \%$. \\
\hline & Snowfall and wet pavement conditions decrease speed by $13 \%$ and capacity by $11 \%$. \\
\hline & $\begin{array}{l}\text { When "wet and slushy" conditions exist, speed declines by } 25 \% \text { and capacity drops by } \\
18 \% \text {. }\end{array}$ \\
\hline & $\begin{array}{l}\text { When travel lanes are "slushy," speed is reduced by } 30 \% \text { and capacity decreases by } \\
18 \% \text {. }\end{array}$ \\
\hline & Snowfall and snow-covered pavement conditions reduce capacity by $20 \%$. \\
\hline
\end{tabular}

* These factors are no longer posted on the Road Weather Management Program's web site.

The estimates in the above table do not cover all weather types used in this study. Therefore, it was necessary to make some assumptions. For example, it was assumed that fog impacts would be the same as for situations where visibility on rural freeways was less than 530 feet. These values were used for all highway types. Furthermore, it was assumed that the effects of ice were the same as those for heavy snow.

The above table provides mobility reduction estimates for heavy and light precipitation, although it does not quantitatively define the differences between "heavy" and "light." This study assumes rain or snow falling at a rate of 1 inch or more per hour to be heavy, and assumes other amounts are light. The highway capacity and speed reduction impacts of adverse weather conditions used in the study are summarized below.

Table 24. Speed and capacity adjustment factors used in TLC2

\begin{tabular}{l|r|r|r|r|r|r|r|r}
\hline \multirow{2}{*}{$\begin{array}{c}\text { Weather } \\
\text { condition }\end{array}$} & \multicolumn{9}{|c}{ Highway type } \\
\cline { 2 - 8 } & Urban freeway & \multicolumn{2}{|c}{ Rural freeway } & \multicolumn{2}{c}{ Urban arterial } & \multicolumn{2}{c}{ Rural arterial } \\
\cline { 2 - 8 } & Capacity & Speed & Capacity & \multicolumn{1}{c}{ Speed } & Capacity & Speed & Capacity & Speed \\
\hline Light rain & $4 \%$ & $10 \%$ & $4 \%$ & $10 \%$ & $6 \%$ & $10 \%$ & $6 \%$ & $10 \%$ \\
\hline Heavy rain & $8 \%$ & $16 \%$ & $10 \%$ & $25 \%$ & $6 \%$ & $10 \%$ & $6 \%$ & $10 \%$ \\
\hline Light snow & $7.5 \%$ & $15 \%$ & $7.5 \%$ & $15 \%$ & $11 \%$ & $13 \%$ & $11 \%$ & $13 \%$ \\
\hline Heavy snow & $27.5 \%$ & $38 \%$ & $27.5 \%$ & $38 \%$ & $18 \%$ & $25 \%$ & $18 \%$ & $25 \%$ \\
\hline Fog & $6 \%$ & $13 \%$ & $6 \%$ & $13 \%$ & $6 \%$ & $13 \%$ & $6 \%$ & $13 \%$ \\
\hline Ice & $27.5 \%$ & $38 \%$ & $27.5 \%$ & $38 \%$ & $18 \%$ & $25 \%$ & $18 \%$ & $25 \%$ \\
\hline
\end{tabular}

To accurately estimate capacity reduction, the time period during which each event impaired visibility and affected roadway conditions must also be determined. Unfortunately, there is no available national- 
level data on the length of time weather events impacted roadways. Therefore, the duration of the weather event was used as a surrogate for the duration of the capacity loss. This assumption is valid for events that only affect visibility, such as fog. However, it correlates less closely with capacity reductions realized from adverse pavement conditions, since events such as rain, snowfall, and ice can impede traffic well after precipitation stops. Furthermore, if the road temperature is sufficient, snow may not stick on the pavement and may cause little capacity reduction. Therefore, this assumption likely leads to an underestimate of capacity reduction and delay.

\subsubsection{Estimating Delay}

All methodologies used to estimate delays are based on procedures outlined in Highway Capacity Manual 2000 (TRB 2000). These are described in the sections below.

\section{Rural/Urban Interstates and Urban Other Expressways}

One of the major traffic operation parameters required for calculating traffic delay is average speed. The average speed information can be determined by the speed-volume relationship. The traffic volume by hour of the day and day of the week can be estimated based on the AADT and the k-factor (peak-hour traffic volume factor) from HPMS.

Traffic flow on a freeway segment can be categorized into three flow types: under-saturated flow, queue discharge flow, and over-saturated flow (Fig. 15). Each flow type is defined within general speed-flow-density ranges, and each represents different conditions on the freeway.

- Under-saturated flow represents traffic flow which is unaffected by upstream or downstream conditions. This flow regime is generally defined within a speed range of 90 to 120 kilometers per hour (kph) at low to moderate flows and between 70 and $100 \mathrm{kph}$ at high flow rates.

- Queue discharge flow represents traffic flow that has just passed through a bottleneck and is accelerating back up to the free-flow speed of the freeway. Queue discharge flow is characterized by relatively stable flow as long as the effects of another bottleneck downstream are not present. This flow type is generally defined within a narrow range of flows, 2,000-2,300 pcphpl, with speeds typically ranging from $55 \mathrm{kph}$ up to the free-flow speed of the freeway segment. Lower speeds are typically observed just downstream of the bottleneck. Depending upon horizontal and vertical alignments, queue discharge flow usually accelerates back up to the free-flow speed of the facility within 1 to 2 kilometers downstream from the bottleneck. Studies suggest that the queue discharge flow rate from the bottleneck is lower than the maximum flows observed prior to breakdown. A typical value for this drop in flow rate is approximately 5 percent.

- Over-saturated flow represents traffic flow that is influenced by the effects of a downstream bottleneck. Traffic flow in the congested regime can vary over a broad range of flows and speeds depending upon the severity of the bottleneck. Queues may extend several kilometers upstream from the bottleneck. Freeway queues differ from queues at intersections in that they are not static, or standing. On freeways, vehicles move slowly through a queue, with periods of both stopping and movement.

Speed-flow and density-flow relationships for a typical basic freeway segment under either base or non-base conditions in which free-flow speed is known are shown in Fig. 16. Recent freeway studies indicate that speed on freeways is insensitive to flow in the low to moderate range. This is reflected in Fig. 16, which shows speed to be constant for flows up to $1,300 \mathrm{pcphpl}$ for a 120-kph free-flow speed. For lower free-flow speeds, the region over which speed is insensitive to flow extends to even higher flow rates. 
Under base traffic and geometric conditions, freeways will operate with capacities as high as 2,400 pcphpl. This capacity is typically achieved on freeways with free-flow speeds of $120 \mathrm{kph}$ or greater. As the free-flow speed decreases, there is a slight decrease in capacity. For example, capacity of a basic freeway segment with a free-flow speed of $90 \mathrm{kph}$ is expected to be approximately $2,250 \mathrm{pcphpl}$.

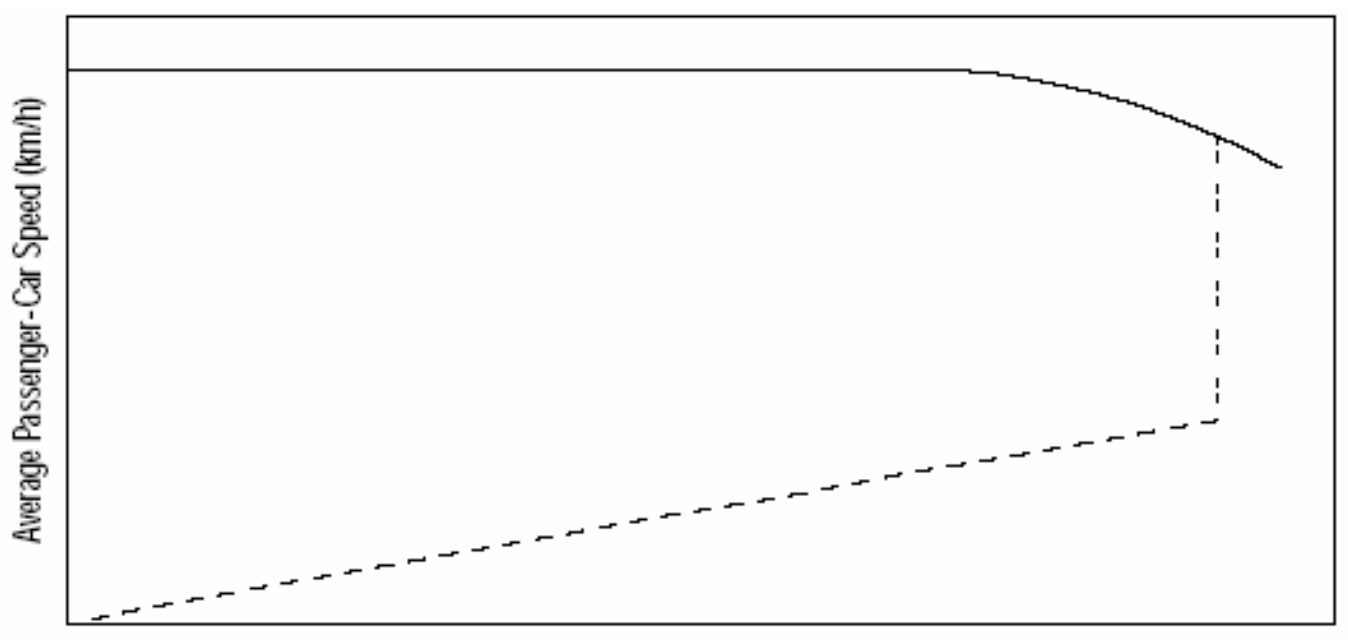

Flow Rate $(\mathrm{pc} / \mathrm{h} / \mathrm{ln})$

Regime 1 (undersaturated)

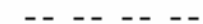

Regime 2 (queue discharge)

Regime 3 (coversaturated)

Fig. 15. Freeway traffic flow types

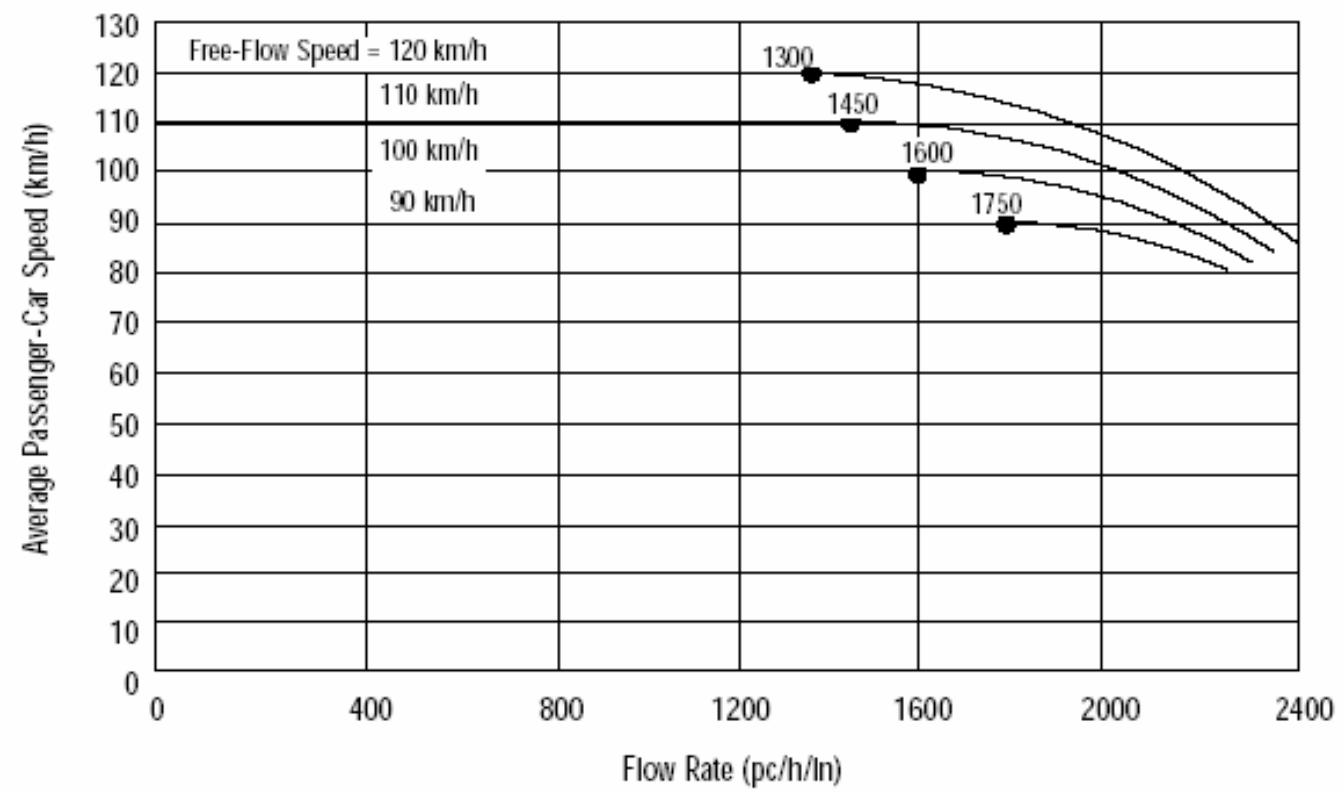

Fig. 16. Speed-flow relationships for a typical basic freeway segment

As indicated in Fig. 16, the point at which an increase in flow rate begins to impact the average passenger car speed varies from 1,300 to 1,750 pcphpl. Speed will be reduced beginning at 1,300 pcphpl for freeway segments with a free-flow speed of $120 \mathrm{kph}$. For facilities with lower free-flow speeds, the average speed begins to diminish at higher flow rates. 
The relationships in Fig. 16 were digitized and transformed into speed volume-to-capacity ratio relationships. Polynomial equations to the fourth order were used and "fitted" to these curves (Equations 14-17 and Fig. 17). These equations made it possible to use a computer program to calculate average speeds based on volume-to-capacity ratio.

Using the speed limit (used as free flow speed), AADT, peak-hour factor, directional flow split (applied to morning and afternoon peak-hours) and peak-hour capacity information from HPMS, the average speed was estimated based on these equations. If the volume-to-capacity ratio was larger than 1 , the queue was tracked, and queue length and queue delay were calculated. The total travel time was calculated as the travel time (based on average speed estimated by one of the abovementioned equations) plus the queue delay.

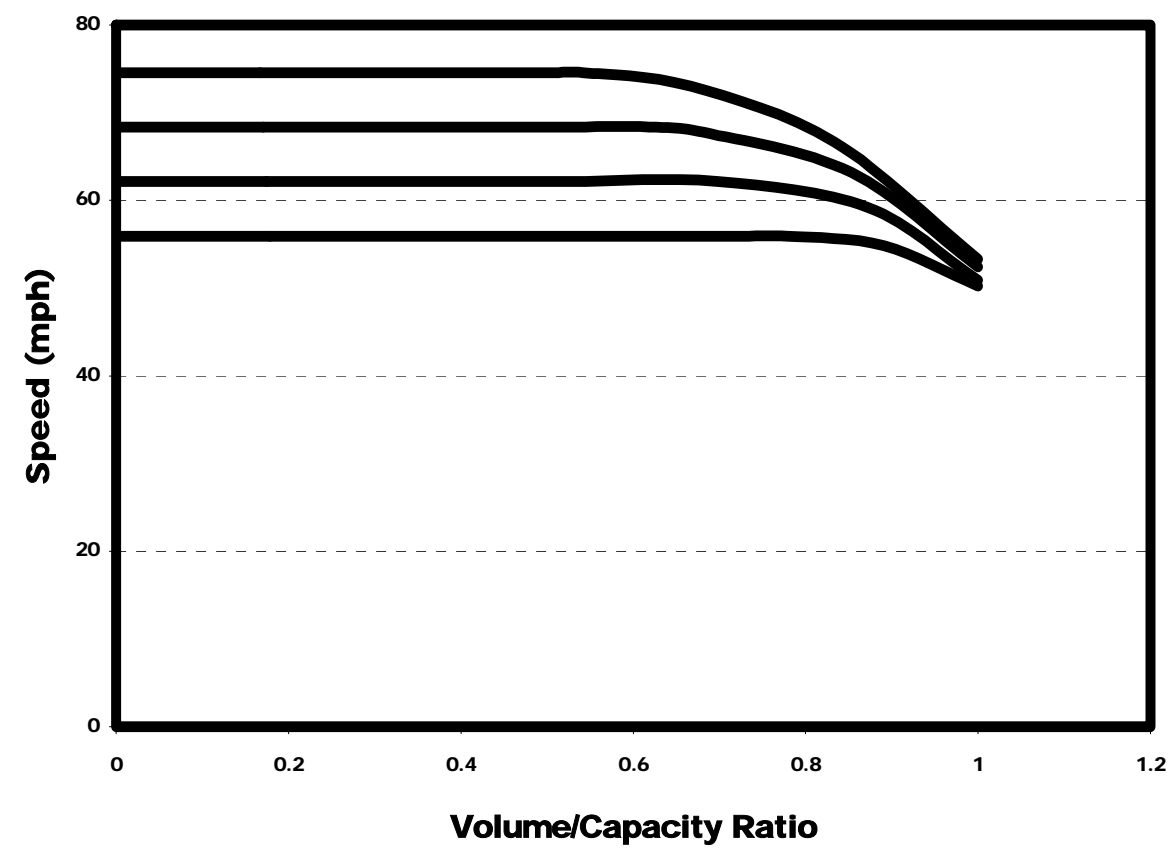

Fig. 17. Speed relationship to volume/capacity ratio for basic freeway segment

Avg Speed $(m p h)_{F F S \approx 75 m p h}=-46.97\left(\frac{v}{c}\right)^{4}+22.72\left(\frac{v}{c}\right)^{3}+5.0909\left(\frac{v}{c}\right)^{2}-2.1844\left(\frac{v}{c}\right)+74.6$

Avg Speed $(m p h)_{F F S \approx 68 m p h}=-87.69\left(\frac{v}{c}\right)^{4}+107.92\left(\frac{v}{c}\right)^{3}-40.719\left(\frac{v}{c}\right)^{2}+4.5651\left(\frac{v}{c}\right)+68.345$

Avg Speed $(m p h)_{F F S \approx 60 m p h}=-111.01\left(\frac{v}{c}\right)^{4}+164.11\left(\frac{v}{c}\right)^{3}-74.212\left(\frac{v}{c}\right)^{2}-9.9859\left(\frac{v}{c}\right)+60.091$

Avg Speed $(m p h)_{F F S \approx 55 m p h}=-79.645\left(\frac{v}{c}\right)^{4}+127.36\left(\frac{v}{c}\right)^{3}-62.256\left(\frac{v}{c}\right)^{2}-9.0488\left(\frac{v}{c}\right)+55.87$

\section{Rural/Urban Other Major Principal Arterials}

Intersections without Signals or Stop Signs: The speed-flow relationships for a typical uninterrupted-flow segment on a multilane highway under either base or non-base conditions in which 
free-flow speed is known are shown in Fig. 18. The operating characteristics for a multilane highway may be slightly lower than for a freeway because drivers on multilane highways allow for potential conflicts with turning traffic, even when there are no access points in the immediate vicinity.

As indicated in Fig. 18, the speed of traffic on a multilane highway is insensitive to traffic volume up to a flow rate of 1,400 pcphpl. The exhibit shows that the capacity of a multilane highway under base conditions is 2,200 pcphpl for highways with a 100-kph free-flow speed. For flow rates from 1,400 to 2,200 pcphpl, the speed on a multilane highway with a 100-kph free-flow speed drops $12 \mathrm{kph}$.

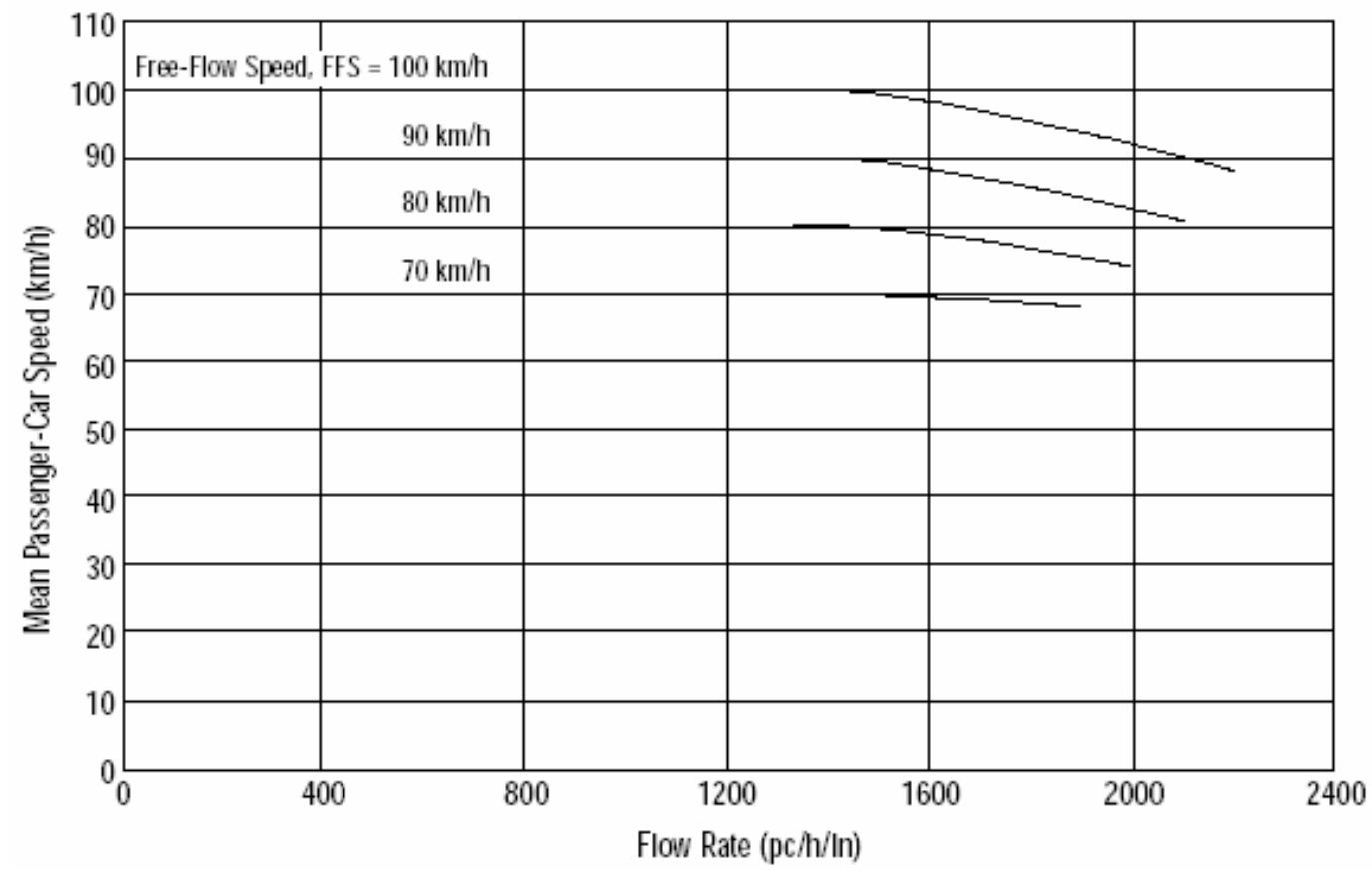

Fig. 18. Speed-flow relationships on multi-lane freeways

The capacity value of 2,200 pcphpl is representative of the maximum 15-minute flow rate that can be accommodated under base conditions for highways with a free-flow speed of $100 \mathrm{kph}$. Actual capacities on specific multilane highway sections may vary from this value.

The relationships presented in Fig. 18 were digitized, polynomial equations to the fourth order were "fitted" to the displayed curves (Equations 18-21 and Fig. 19). These equations made it possible to use a computer program to calculate average speeds based on volume-to-capacity ratio.

$\operatorname{Avg} \operatorname{Speed}(\mathrm{mph})_{\mathrm{FFS}=62 \mathrm{mph}}=-2.2238\left(\frac{v}{c}\right)^{4}-23.011\left(\frac{v}{c}\right)^{3}+21.701\left(\frac{v}{c}\right)^{2}-4.3551\left(\frac{v}{c}\right)+62.184$

$\operatorname{Avg} \operatorname{Speed}(\mathrm{mph})_{\mathrm{FFS}=55 \mathrm{mph}}=-6.4985\left(\frac{v}{c}\right)^{4}-10.795\left(\frac{v}{c}\right)^{3}+14.347\left(\frac{v}{c}\right)^{2}-3.226\left(\frac{v}{c}\right)+55.962$

$\operatorname{Avg} \operatorname{Speed}(\mathrm{mph})_{\mathrm{FFS}=50 \mathrm{mph}}=0.246\left(\frac{v}{c}\right)^{4}-14.159\left(\frac{v}{c}\right)^{3}+12.523\left(\frac{v}{c}\right)^{2}-2.4503\left(\frac{v}{c}\right)+49.729$ 
$\operatorname{Avg} \operatorname{Speed}(\mathrm{mph})_{\mathrm{FFS}=44 \mathrm{mph}}=-13.636\left(\frac{v}{c}\right)^{4}+20.225\left(\frac{v}{c}\right)^{3}-9.1272\left(\frac{v}{c}\right)^{2}+1.2136\left(\frac{v}{c}\right)+43.496$

Similar to the method used for freeways, the average speed was estimated using these equations and the speed limit (used as free flow speed), AADT, peak-hour factor, directional flow split (applied to morning and afternoon peak-hours) and peak-hour capacity information from HPMS. If the volume-to-capacity ratio was larger than 1 , the queue was tracked, and queue length and queue delay were calculated. The total travel time was calculated as the travel time (based on average speed estimated by one of the abovementioned equations) plus the queue delay.

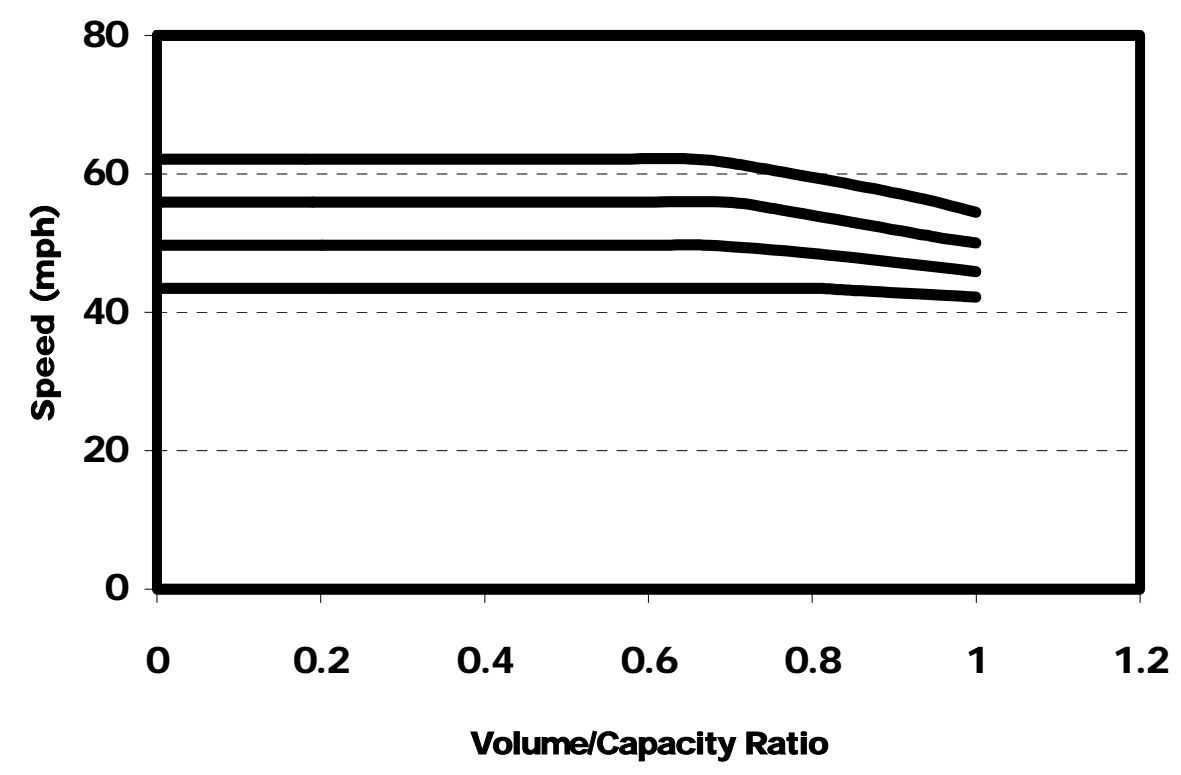

Fig. 19. Speed relationship to volume/capacity ratio for multi-lane freeways

Signal-Controlled Intersections: For principal arterials with signal-controlled intersections, the capacity for the arterial segment was calculated as the capacity at the signal-controlled intersections. Thus, average speed was dominated by the delay time at these signal-controlled intersections.

The methodology described in Section 7.1.2, "Estimating Total Delay for Signal-Controlled Intersections," was used to estimate total delay time at signal-controlled intersections during adverse weather conditions. If the volume-to-capacity ratio was larger than 1 , queue was tracked and queue length and queue delay were calculated. The total travel time was calculated as the sum of the travel time (based on free flow speed), signal-controlled intersection delay time, and queue delay.

Stop-Sign-Controlled Intersections: For principal arterials with stop-sign-controlled intersections, the capacity for the arterial segment was calculated as the capacity of the stop-sign-controlled intersections. Thus, average speed was dominated by the delay time at these intersections.

The delay experienced by a motorist is made up of a number of factors that relate to control, geometry, traffic, and incidents. Total delay is the difference between the travel time actually experienced and the reference travel time that would result during ideal conditions, in the absence of incident, control, traffic, or geometric delay. This study quantifies only that portion of total delay attributed to traffic control measures (i.e., either traffic signals or stop signs). This delay is called control delay. Control delay includes initial deceleration delay, queue move-up time, stopped delay, and final acceleration delay. With 
respect to field measurements, control delay is defined as the total elapsed time from when a vehicle stops at the end of the queue until the vehicle departs from the stop line. This total elapsed time includes the time required for the vehicle to travel from the last-in-queue position to the first-in-queue position, including deceleration of vehicles from free-flow speed to the speed of vehicles in queue.

Average control delay for any particular minor movement is a function of the capacity of the approach and the degree of saturation. The analytical model used to estimate control delay (Equation 22) assumes that the demand is less than capacity for the period of analysis. In situations where the degree of saturation is greater than about 0.9 , average control delay is significantly affected by the length of the analysis period. In most cases, the recommended analysis period is 15 minutes. If demand exceeds capacity during a 15-minute period, the delay results calculated by the procedure may not be accurate. In this case, the period of analysis should be lengthened to include the period of over-saturation.

The constant value of 5 seconds/vehicle is included in Equation 22 to account for the deceleration of vehicles from free-flow speed to the speed of vehicles in queue and the acceleration of vehicles from the stop line to free-flow speed.

$$
d=\frac{3,600}{c_{x}}+900 T\left(\frac{v_{x}}{c_{x}}-1+\sqrt{\left(\frac{v_{x}}{c_{x}}-1\right)^{2}+\frac{\left(\frac{3,600}{c_{x}}\right)\left(\frac{v_{x}}{c_{x}}\right)}{450 T}}\right)+5
$$

where

$$
\begin{aligned}
& \mathrm{d}=\text { stop sight control delay in seconds/vehicle } \\
& \mathrm{v}_{\mathrm{x}}=\text { flow rate for movement } \mathrm{x} \text { in vehicles/hour } \\
& \mathrm{c}_{\mathrm{x}}=\text { capacity of movement } \mathrm{x} \text { in vehicles/hour } \\
& \mathrm{T}=\text { analysis time period in hours }(\mathrm{T}=0.25 \text { for a } 15 \text {-minute period })
\end{aligned}
$$

If the volume-to-capacity ratio was larger than 1, the queue was tracked, and queue length and queue delay were calculated. The total travel time was calculated as the travel time (based on free flow speed, stop-sign-controlled intersection delay time) plus the queue delay.

To estimate the delay due to adverse weather conditions, the normal delay (congestion delay without the adverse weather condition) for roadways in each county during the time of the event was estimated. ${ }^{7}$ The delay with adverse weather conditions for roadways in each county during the time of the event was then estimated. This was accomplished by repeating the same delay estimation step with the appropriate capacity reduction for the event type. If the link average speed was greater than the typical travel speed for that event, the delay was calculated at that speed. Otherwise, the delay was not adjusted. The delay induced by adverse weather conditions for each county was calculated as the difference in the estimated delay with the adverse weather condition and the estimated delay without the adverse weather condition. The delay induced by adverse weather conditions was then calculated for each weather zone (public

\footnotetext{
${ }^{7}$ It should be noted that the event duration was assumed to be the time period during which the weather event took place. For rain, snow, and ice, the duration would include only the time during which precipitation fell; it would not include the impact of the precipitation on the ground after it stopped falling.
} 
forecast zone or Thiessen polygon) as the sum of the delay for all involved counties multiplied by the percentage of each county's major arterial mileage lying within that zone. These estimates were summed to produce a national total.

\subsection{RESULTS}

The methodology and data used in TLC2 to estimate weather-related capacity reductions and delay was modified from the original TLC study. Therefore, this section includes the revised weather-related delay estimates, along with an illustration of the difference between the estimates produced by both methods and an explanation as to why some of the revised estimates are considerably different from those in the original study.

\subsubsection{TLC2 Results}

The TLC2 study estimated that, in 1999, rain, fog, snow, and icy conditions temporarily reduced capacity on freeways and principal arterials by approximately 20.9 billion vehicles. This resulted in an estimated 330.1 million vehicle-hours of delay. Rain accounted for most of the delay from adverse weather (71 percent), followed by ice (14 percent), snow (13 percent), and fog ( 2 percent). Urban areas experienced 92 percent of the delay from weather events, with most of this delay experienced on principal arterials. As shown in the figures below, capacity reductions were less likely to translate into delays in rural areas.

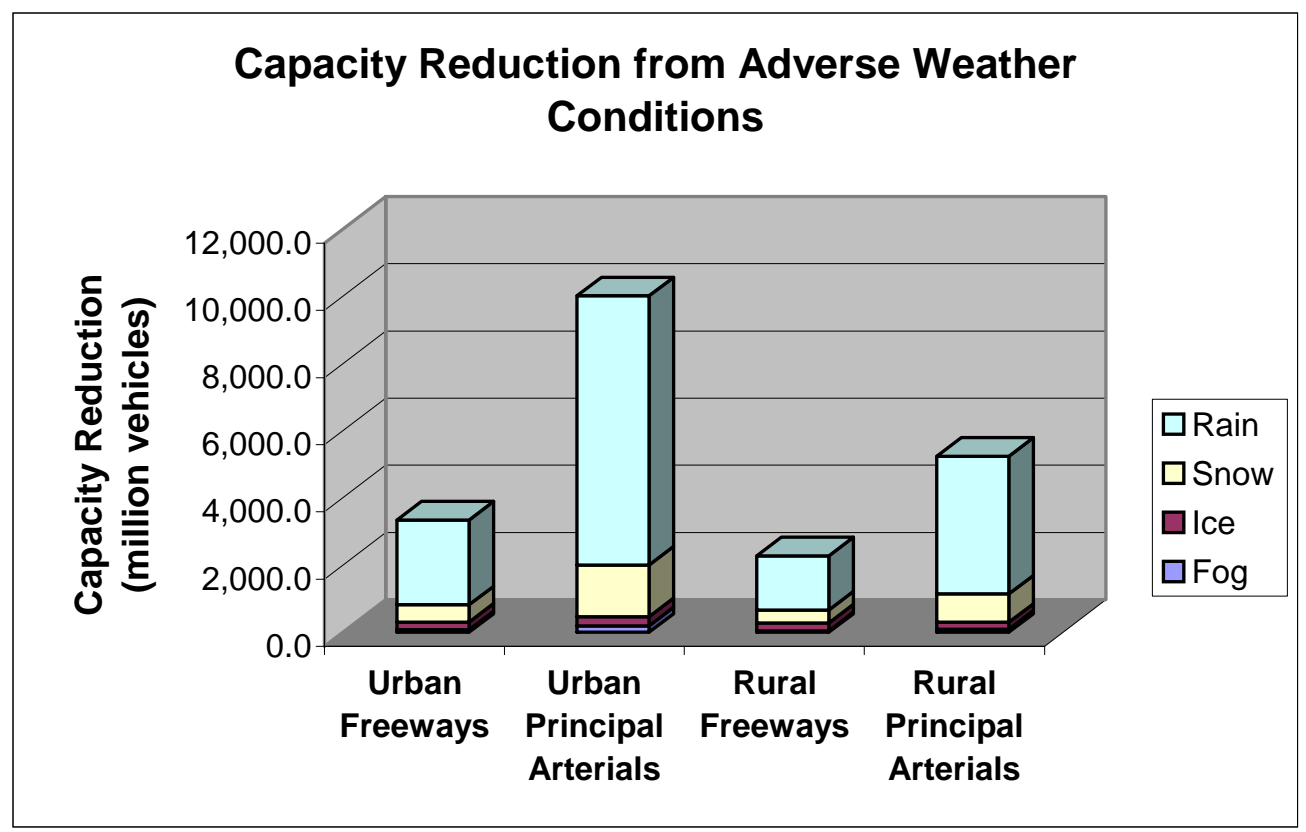

Fig. 20. Most weather-related capacity reduction occurred from rain on urban and rural arterials. 


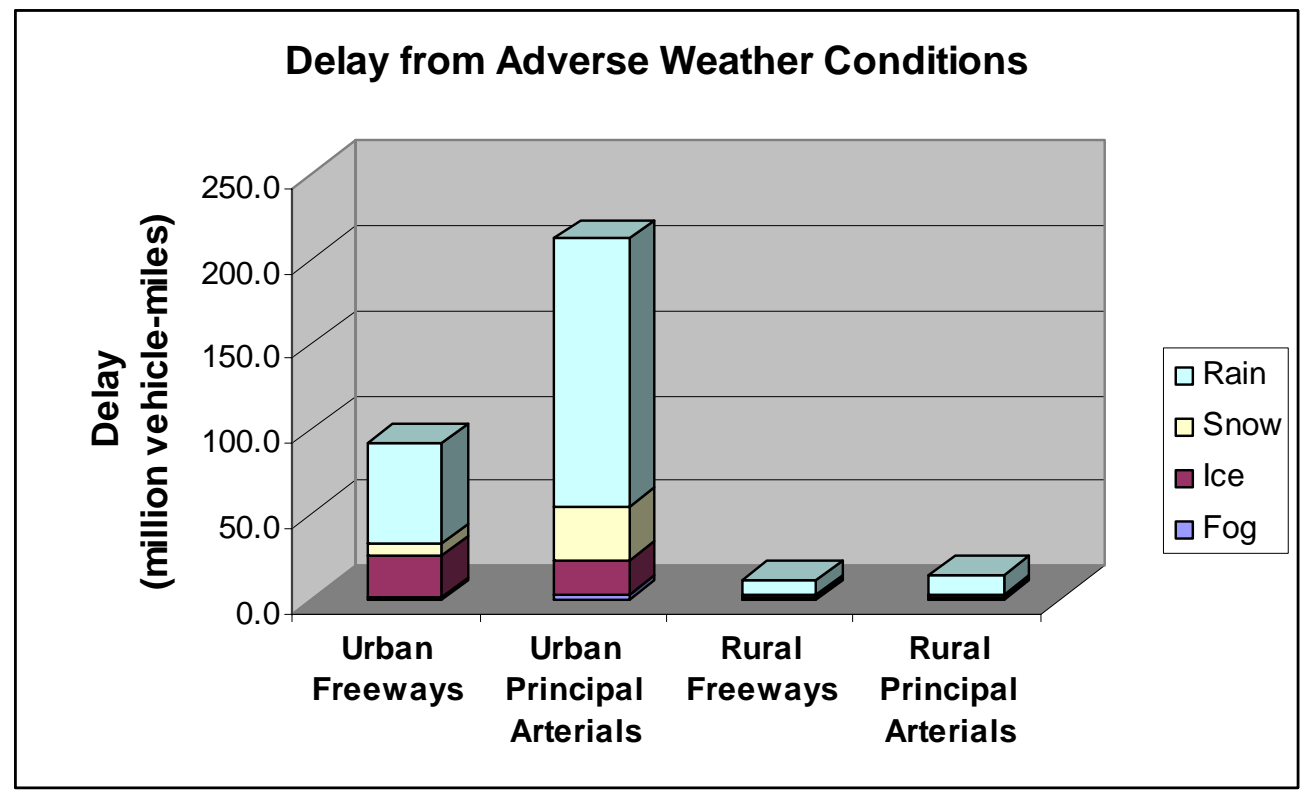

Fig. 21. Most weather-related delay was experienced in urban areas

Table 25. Summary of capacity loss \& delay due to adverse weather conditions, 1999

\begin{tabular}{lrrrrr}
\hline \multicolumn{1}{c}{ Highway type } & Fog & Ice & Snow & \multicolumn{1}{c}{ Rain } & \multicolumn{1}{c}{ All } \\
\hline & \multicolumn{7}{c}{} \\
CAPACITY REDUCTION & (MILLION & VEHICLES) & & \\
\hline Urban freeways & 75.2 & 225.5 & 517.0 & $2,520.9$ & $3,338.5$ \\
Urban principal arterials & 191.2 & 274.5 & $1,541.1$ & $8,009.1$ & $10,016.0$ \\
Rural freeways & 46.0 & 230.1 & 384.6 & $1,614.5$ & $2,275.2$ \\
Rural principal arterials & 97.9 & 199.4 & 843.7 & $4,093.9$ & $5,235.0$ \\
\hline Total & 410.3 & 929.5 & $3,286.4$ & $16,238.4$ & $20,864.6$ \\
\hline \multicolumn{7}{c}{} & & & & \\
DELAY (MILLION VEHICLE-HOURS) & 1.6 & 23.8 & 8.4 & 58.0 & 91.8 \\
\hline Urban freeways & 3.4 & 19.5 & 32.2 & 156.7 & 211.8 \\
Urban principal arterials & 0.2 & 0.9 & 1.7 & 9.3 & 12.2 \\
Rural freeways & 0.5 & 0.5 & 1.7 & 11.7 & 14.3 \\
Rural principal arterials & 5.8 & 44.8 & 43.8 & 235.7 & 330.1 \\
\hline Total
\end{tabular}


Table 26. Detailed estimates of capacity loss due to adverse weather conditions, 1999

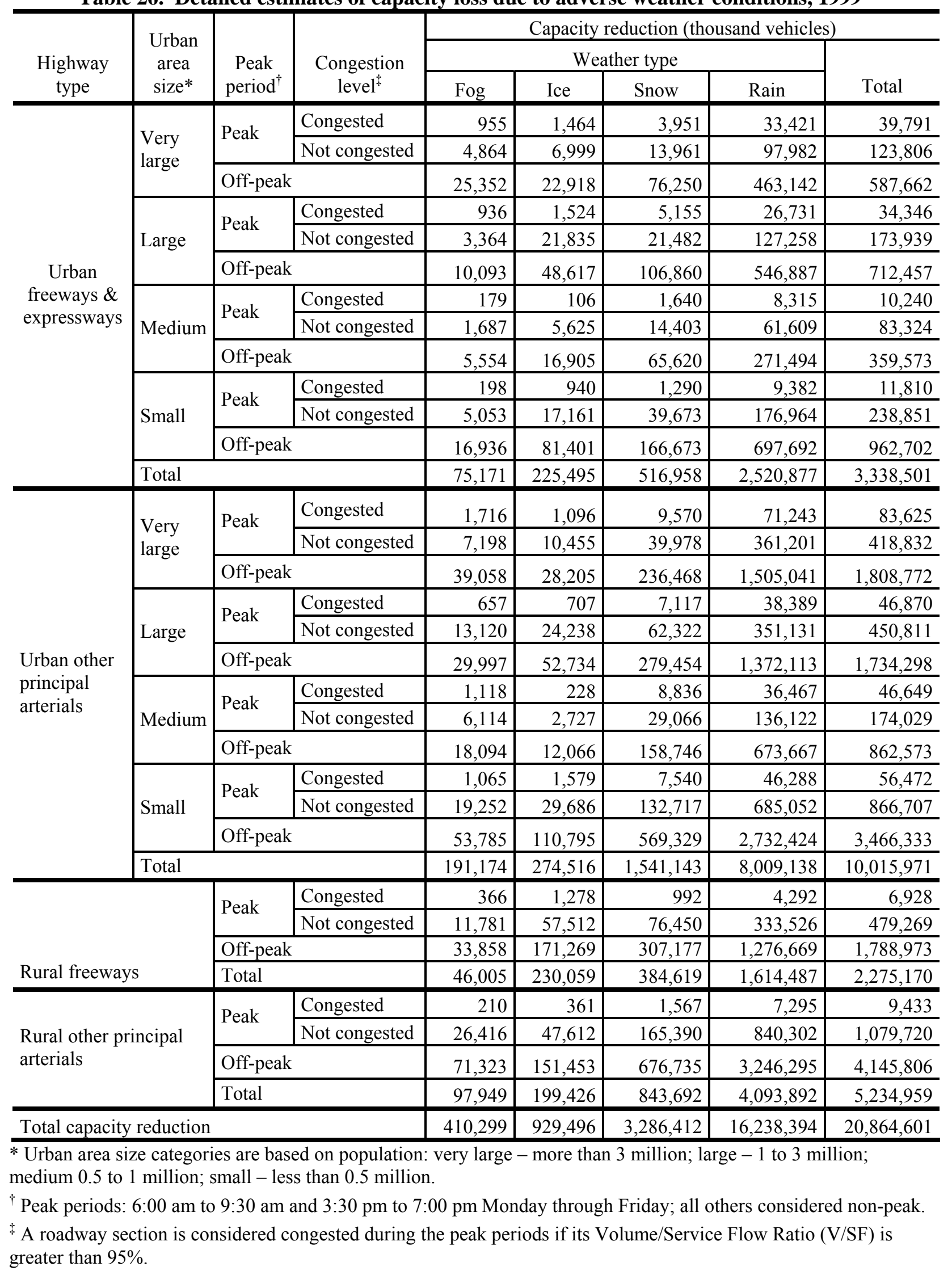


Table 27. Detailed estimates of delay due to adverse weather conditions, 1999

\begin{tabular}{|c|c|c|c|c|c|c|c|c|}
\hline \multirow[b]{3}{*}{ Highway type } & \multirow{3}{*}{$\begin{array}{l}\text { Urban } \\
\text { area } \\
\text { size* }\end{array}$} & \multirow{3}{*}{$\begin{array}{c}\text { Peak } \\
\text { period }^{\dagger}\end{array}$} & \multirow{3}{*}{$\begin{array}{c}\text { Congestion } \\
\text { level }^{*}\end{array}$} & \multicolumn{5}{|c|}{ Delay (thousand vehicle-hours) } \\
\hline & & & & \multicolumn{4}{|c|}{ Weather type } & \multirow[b]{2}{*}{ Total } \\
\hline & & & & Fog & Ice & Snow & Rain & \\
\hline \multirow{13}{*}{$\begin{array}{l}\text { Urban } \\
\text { freeways \& } \\
\text { expressways }\end{array}$} & \multirow{3}{*}{$\begin{array}{l}\text { Very } \\
\text { large }\end{array}$} & \multirow{2}{*}{ Peak } & Congested & 49 & 2,653 & 1,175 & 9,935 & 13,812 \\
\hline & & & Not Congested & 87 & 1,282 & 345 & 2,817 & 4,531 \\
\hline & & \multicolumn{2}{|l|}{ Off-Peak } & 164 & 6,366 & 1,846 & 13,458 & 21,834 \\
\hline & \multirow{3}{*}{ Large } & \multirow{2}{*}{ Peak } & Congested & 54 & 3,456 & 582 & 5,258 & 9,350 \\
\hline & & & Not Congested & 47 & 803 & 365 & 3,022 & 4,236 \\
\hline & & \multicolumn{2}{|l|}{ Off-Peak } & 74 & 8,062 & 1,380 & 9,456 & 18,973 \\
\hline & \multirow{3}{*}{ Medium } & \multirow{2}{*}{ Peak } & Congested & 393 & 122 & 277 & 1,338 & 2,130 \\
\hline & & & Not Congested & 49 & 71 & 232 & 1,203 & 1,556 \\
\hline & & \multicolumn{2}{|l|}{ Off-Peak } & 569 & 172 & 664 & 3,295 & 4,700 \\
\hline & \multirow{3}{*}{ Small } & \multirow{2}{*}{ Peak } & Congested & 5 & 111 & 71 & 660 & 848 \\
\hline & & & Not Congested & 53 & 185 & 421 & 2,351 & 3,011 \\
\hline & & \multicolumn{2}{|l|}{ Off-Peak } & 75 & 537 & 994 & 5,219 & 6,825 \\
\hline & Total & & & 1,619 & 23,822 & 8,352 & 58,012 & 91,805 \\
\hline \multirow{13}{*}{$\begin{array}{l}\text { Urban other } \\
\text { principal } \\
\text { arterials }\end{array}$} & \multirow{3}{*}{$\begin{array}{l}\text { Very } \\
\text { large }\end{array}$} & \multirow{2}{*}{ Peak } & Congested & 410 & 1,227 & 2,921 & 20,762 & 25,319 \\
\hline & & & Not Congested & 134 & 333 & 1,459 & 5,158 & 7,084 \\
\hline & & \multicolumn{2}{|l|}{ Off-Peak } & 689 & 3,333 & 8,214 & 37,415 & 49,651 \\
\hline & \multirow{3}{*}{ Large } & \multirow{2}{*}{ Peak } & Congested & 268 & 1,532 & 1,366 & 8,568 & 11,733 \\
\hline & & & Not Congested & 118 & 270 & 381 & 5,026 & 5,795 \\
\hline & & Off-Peak & & 214 & 4,085 & 3,964 & 22,115 & 30,379 \\
\hline & & Peak & Congested & 61 & 62 & 1,090 & 3,121 & 4,333 \\
\hline & Medium & reak & Not Congested & 104 & 499 & 1,039 & 3,361 & 5,003 \\
\hline & & Off-Peak & & 121 & 891 & 2,710 & 8,187 & 11,908 \\
\hline & & Peak & Congested & 404 & 1,455 & 1,741 & 8,434 & 12,034 \\
\hline & Small & & Not Congested & 320 & 816 & 1,666 & 8,769 & 11,571 \\
\hline & & Off-Peak & & 604 & 4,979 & 5,613 & 25,798 & 36,995 \\
\hline & Total & & & 3,446 & 19,480 & 32,164 & 156,714 & 211,805 \\
\hline & & Peak & Congested & 7 & 45 & 34 & 115 & 201 \\
\hline Rural freewavs & & & Not Congested & 102 & 315 & 462 & 2,776 & 3,656 \\
\hline Nurar ile ways & & Off-Peak & & 135 & 575 & 1,173 & 6,448 & 8,330 \\
\hline & & Total & & 244 & 934 & 1,669 & 9,340 & 12,187 \\
\hline & & Peak & Congested & 11 & 27 & 35 & 228 & 301 \\
\hline Rural other prin & pal & Геак & Not Congested & 189 & 162 & 428 & 3,397 & 4,176 \\
\hline arterials & & Off-Peak & & 276 & 346 & 1,194 & 8,052 & 9,868 \\
\hline & & Total & & 476 & 536 & 1,657 & 11,677 & 14,346 \\
\hline Total delay & & & & 5,785 & 44,772 & 43,842 & 235,743 & 330,142 \\
\hline
\end{tabular}

* Urban area size categories are based on population: very large - more than 3 million; large - 1 to 3 million; medium 0.5 to 1 million; small - less than 0.5 million.

${ }^{\dagger}$ Peak periods: 6:00 am to 9:30 am and 3:30 pm to 7:00 pm Monday through Friday; all others considered non-peak.

$\$$ A roadway section is considered congested during the peak periods if its Volume/Service Flow Ratio (V/SF) is greater than $95 \%$. 


\subsubsection{Comparing TLC and TLC2 Results}

The TLC2 study's estimated capacity reduction and delay resulting from snow and fog are significantly different from those estimated by the initial TLC study. This is primarily due to two factors. First, different speed reduction factors were used in these studies. In TLC2, speed reduction factors found on the FHWA Road Weather Management Program website were used in lieu of those derived from the Highway Capacity Manual. Second, the Road Weather Management Program's site provided separate factors for "heavy" and "light" weather conditions, whereas the sources used for the initial TLC only applied to "heavy" conditions. In the first TLC study, all events were assumed to be heavy. Conversely, a significant number of the weather events in the TLC2 study were characterized as light fog or light snow. Therefore, these lower speed reduction estimates affect a large number of the weather events within the study, resulting in much lower delay estimates for these kinds of events and for the overall delay from weather. These are the only differences in the methodologies for estimating impacts from fog, snow, and ice.

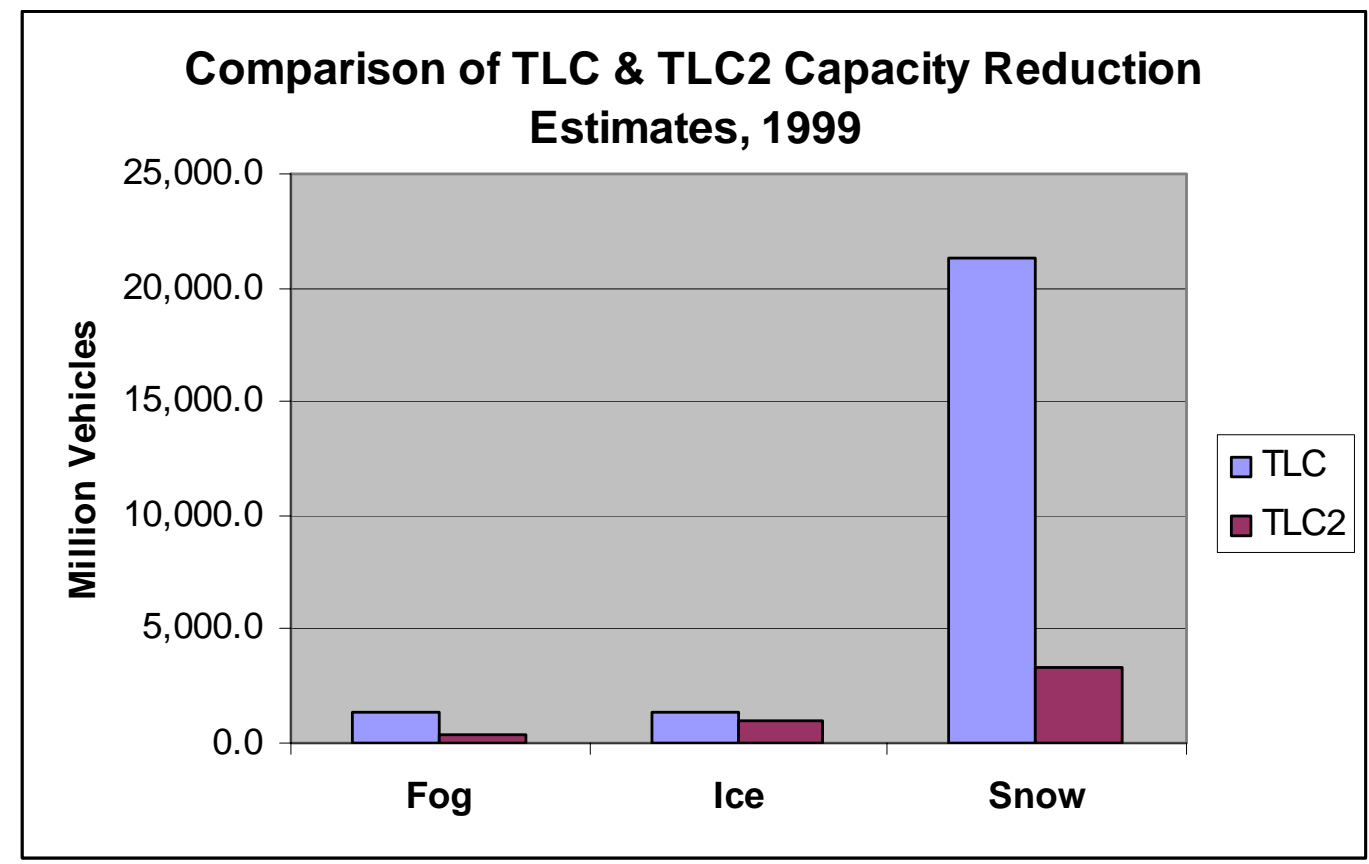

Fig. 22. Capacity reductions due to fog and snow estimated by TLC2 were significantly lower than those estimated by the initial TLC study. 


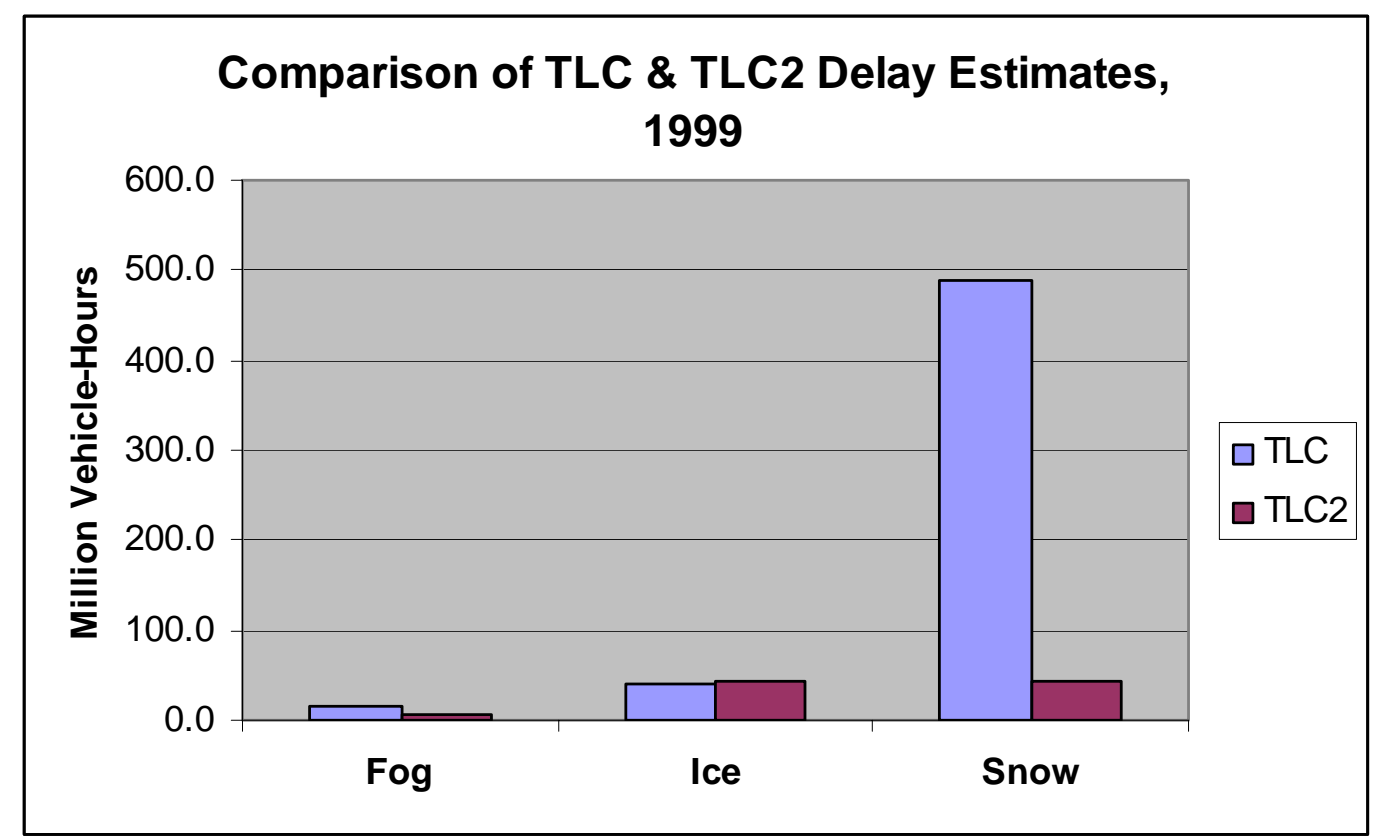

Fig. 23. Delays due to fog and snow estimated by TLC2 were significantly lower than those estimated by the initial TLC study.

\subsection{RELIABILITY}

\subsubsection{Methodology}

Established analytical procedures were used to map weather information to HPMS data. However, due to the complex nature of local weather patterns and the factors that determine them, these methods are qualified as having a medium degree of confidence.

Methodologies from the Highway Capacity Manual were used to estimate capacity loss due to speed reductions and delay due to capacity reductions. These methodologies have evolved over the years and have been updated and enhanced continuously when new information or methodologies are made available. They are well established and accepted within the traffic engineering community. These methodologies are qualified as having a high degree of confidence.

\subsubsection{Data \& Key Assumptions}

The "Hourly Precipitation Data (HPD) TD3240" and "Cooperative Summary of Day TD3200" databases compiled by National Weather Service are qualified as having high degree of confidence. However, it should be noted that precipitation gauges are not as accurate for frozen precipitation and, therefore, may slightly underestimate snow measurements.

The "Storm Data and Unusual Weather Phenomena" database compiled by the National Climatic Data Center (NCDC) is qualified as having a high degree of confidence. However, as noted previously, the database only includes storms and other important climatologic events having sufficient intensity to cause injuries, loss of life, and/or significant property damage ( $\$ 1,000$ or more). Less severe events can still reduce capacity and cause delay - this is especially true for fog, which can cause delay even if no significant monetary damages result. However, due to data limitations, these events are not modeled and their overall contribution to delay is not known. 
Adverse weather impacts on capacity and operating speeds are based on information acquired from the FHWA Office of Operations, Road Weather Management Program web site. However, this information was not collected by FHWA, and FHWA has a low degree of confidence in its reliability.

Several assumptions were made in modeling weather and its impacts that have a significant influence on the reliability of these capacity loss and delay estimates.

- The TLC2 study assumes that weather-related impacts occur only during the time that the event occurs (for rain, ice, and show, this would be the duration that precipitation fell). While this relationship rarely exists, especially for ice- and snow-related events that can affect capacity for hours or days after precipitation falls, there is no national-level data on how long roadways were affected by weather events. In addition, the duration of impacts is quite localized and depends upon factors other than the weather itself, such as the availability and use of equipment to prevent or mitigate snow and ice impacts. Therefore, in the absence of data, we have decided to limit the duration of impact to the duration of precipitation, the only part of the weather event for which data was available. Therefore, this assumption is accorded a low degree of confidence for ice and snow.

- The precipitation data does not include specific information about the precipitation form (rain, snow, etc.). Therefore, the precipitation type (rain vs. snow) was estimated based on temperature: at a temperature of $32^{\circ} \mathrm{F}$ or less, precipitation was assumed to be in the form of snow. This assumption is accorded a medium degree of confidence.

- Capacity reduction estimates for snow were used as a surrogate for ice-related events since no estimates were available for ice. It was assumed that the impacts of these events were at least similar in nature. While ice likely reduces capacity more than snow, snow-related capacity reductions were used in keeping with the study's philosophy of erring on the conservative side.

- The Road Weather Management Program data provides speed reduction and capacity reduction data for "heavy" and "light" weather conditions, but the definitions of heavy and light are not quantified. Therefore, any precipitation falling at a rate of over 1 inch per hour was classified as heavy. This assumption is accorded a medium degree of confidence.

The same traffic demand data and surface street assumptions used to estimate crash delays were used for breakdowns. Therefore, the same caveats apply: traffic demand data is accorded a low level of confidence and surface street characteristics are accorded a medium level of confidence (see section 3.3.2).

As indicated at the beginning of this report, TLC2 does not consider the impact of rescheduled or canceled trips, although they have a significant impact on demand in some cases. Also, a number of weather events that can cause delay were not included in the report due to data limitations and/or limitations in time and funding. These include solar glare, high winds, dust storms, flash flooding, and melting and re-freezing of ice and snow, along with catastrophic events such as hurricanes and floods. Finally, the increased likelihood of crashes and disabled vehicles is not modeled nor are the additive impacts of these events occurring during a weather event.

The weather-related estimates should be viewed in the context of these limitations. 


\section{SUB-OPTIMAL SIGNAL TIMING}

\subsection{METHODOLOGY}

Capacity reduction and delay on principal arterials due to sub-optimal signal control were estimated using a three-step process. The first step was to determine the number and type of signals on principal arterials. The next step was to estimate the total vehicle delay at these intersections, since traffic signals cause delay in comparison to free-flowing traffic. However, most of this delay is un-avoidable. The third step was to estimate the avoidable delay due to sub-optimal signal timing. Delay due to sub-optimal signal timing was estimated as a percent of the total delay based on evidence from the literature. Capacity reduction was calculated in a similar manner. However, since HPMS provides capacity estimates, it was not necessary to estimate intersection capacities.

The primary data source for estimating signal control delay was the HPMS Sample Database. The HPMS Sample Database contains the following information used to calculate traffic signal delays:

- Annual Average Daily Traffic (AADT)

- Directional Factor

- Prevailing Type of Signalization

- Typical Peak Percent Green Time

- Number and Type of At-Grade Signal-Controlled Intersections

- Peak Capacity

- Volume/Service Flow Ratio (V/SF)

Other data sources used in this part of the study are noted in the methodology descriptions that follow.

\subsubsection{Identifying Signal-Controlled Intersections on Principal Arterials}

The HPMS Sample Database provides a count of the number of intersections and traffic controls on the nation's roadways. These include at-grade intersections at entrances to shopping centers, industrial parks, and other large traffic-generating enterprises. The database also provides information on the type of signal control used at each intersection. Expansion factors were applied to the totals from the Sample Database to estimate the number of signal-controlled intersections on principal arterials (see Table 29 in Section 7.2.2).

\subsubsection{Estimating Total Delay for Signal-Controlled Intersections}

Delay at signal-controlled intersections on principal arterials was estimated based on a methodology outlined in the Highway Capacity Manual 2000. According to Chapter 16 in the manual, the average delay per vehicle for a lane group at a controlled intersection is given by the following equation:

$d=d_{1} \times P F+d_{2}+d_{3}$

where

$\mathrm{d}=$ control delay per vehicle (seconds/vehicle)

$\mathrm{d}_{1}=$ uniform control delay, assuming uniform arrivals (seconds/vehicle) 


$$
\begin{aligned}
\mathrm{PF}= & \begin{array}{l}
\text { uniform delay progression adjustment factor that accounts for the effects of signal } \\
\text { progression }
\end{array} \\
\mathrm{d}_{2}= & \begin{array}{l}
\text { incremental delay to account for the effect of random and over-saturation queues, } \\
\text { adjusted for the duration of the analysis period and the type of signal control }
\end{array} \\
& \begin{array}{l}
\text { (seconds/vehicle). This delay component assumes that there is no residual demand for } \\
\text { the lane group at the start of the analysis period. }
\end{array} \\
\mathrm{d}_{3}= & \begin{array}{l}
\text { supplemental delay to account for over-saturation queues that may have existed prior to } \\
\text { the analysis period (seconds/vehicle) }
\end{array}
\end{aligned}
$$

Thus, the total control delay per vehicle was the sum of the uniform control delay, the incremental delay, and the supplemental delay. Each of these was estimated on an hourly basis, using time-of-day

distributions for a week. Daily average delay was calculated and multiplied by 365 to calculate the delay for an entire year. The methods used to estimate each type of delay are described in the following sections.

\section{Uniform Delay}

The next equation gives an estimate of delay, assuming uniform arrivals, stable flow, and no initial queue. It is based on the first term of the delay formulation suggested in the Highway Capacity Manual 2000 and is widely accepted as an accurate depiction of delay for the idealized case of uniform arrivals.

$$
d_{1}=\frac{0.50 C(1-g / C)^{2}}{1-[\min (1, X) g / C]}
$$

where

$$
\begin{aligned}
& \mathrm{d}_{1}=\quad \text { uniform control delay, assuming uniform arrivals (seconds/vehicle) } \\
& \mathrm{C}=\begin{array}{l}
\text { cycle length (seconds). Cycle length is used for pre-timed signal control; average cycle } \\
\text { length is used for estimating actuated control parameters. }
\end{array} \\
& \mathrm{g}=\begin{array}{l}
\text { effective green time for a lane group (seconds). Green time is used for pre-timed signal } \\
\text { control; average lane group effective green time is used for actuated control. }
\end{array} \\
& \mathrm{X}=\quad \text { volume/capacity ratio or degree of saturation for a lane group }
\end{aligned}
$$

Effective green time and capacity were obtained from HPMS Sample Data. The volume data used in the volume/capacity ratio was calculated by applying a time-of-day distribution to the AADT data given in HPMS. For urban intersections, this distribution was based on a study of four cities: San Antonio, Texas; Milwaukee, Wisconsin; San Diego, California; and Seattle, Washington (see footnote 4 on page 17). For rural intersections, the distribution was based on hourly traffic counts in the state of Tennessee (T.DOT). Cycle length was calculated based on a methodology described in Revised Monograph on Traffic Flow Theory (U.S. DOT/FHWA 1997).

As shown in equation 23, uniform delay $\left(\mathrm{d}_{1}\right)$ is adjusted using a uniform delay progression adjustment factor (PF) that accounts for the effects of signal progression. A suggestion of the Highway Capacity Manual 2000 was followed in using a progression adjustment factor based on Arrival Type 3 (AT-3) for uncoordinated lane groups and Arrival Type 4 (AT-4) for coordinated lane groups. These guidelines are provided for planning situations where the arrival characteristics cannot be directly observed. Thus, they should be suitable for our purposes. 


\section{Incremental Delay}

The next equation was used to estimate the incremental delay due to non-uniform arrivals and temporary cycle failures (random delay), as well as delay caused by sustained periods of over-saturation (oversaturation delay). Such delay is sensitive to the degree of saturation of the lane group (X), the duration of the analysis period $(\mathrm{T})$, the capacity of the lane group (c), and the type of signal control, as reflected by the control parameter $(\mathrm{k})$. The equation assumes that there is no unmet demand that causes residual queues at the start of the analysis period $(\mathrm{T})$.

$$
d_{2}=900 T\left[(X-1)+\sqrt{(X-1)^{2}+\frac{8 k l X}{c T}}\right]
$$

where

$$
\begin{aligned}
\mathrm{d}_{2}= & \begin{array}{l}
\text { incremental delay to account for the effect of random and over-saturation queues, } \\
\text { adjusted for the duration of the analysis period and the type of signal control }
\end{array} \\
& \text { (seconds/vehicle). This delay component assumes that there is no residual demand for the } \\
& \text { lane group at the beginning of the analysis period. } \\
\mathrm{T}= & \text { duration of analysis period (hours) } \\
\mathrm{k}= & \text { incremental delay factor that is dependent on controller settings } \\
l= & \text { upstream filtering/metering adjustment factor } \\
\mathrm{c}= & \text { lane group capacity (vehicles/hour) } \\
\mathrm{X}= & \text { lane group volume/capacity ratio or degree of saturation }
\end{aligned}
$$

Since delay was calculated on an hourly basis in this study, T was equal to 1 . The upstream filtering/metering adjustment factor $(l)$ was also set to 1 , because there is no information that would allow it to be calculated. The incremental delay factor $(\mathrm{k})$ was set to 0.5 for pre-timed control, the value suggested by the Highway Capacity Manual 2000. The incremental delay factor for actuated control was based on the extension value and the degree of saturation and ranged from 0.04 to 0.5 . The degree of saturation was taken from HPMS. Since timing plans for each signal are not available, an extension of 3 seconds was assumed for actuated controls. This yielded $\mathrm{k}$ values ranging from 0.11 to 0.50 .

\section{Supplemental Delay}

When a residual demand from a previous time period causes a residual queue to occur at the start of the analysis period (T), additional delay is experienced since the residual queues must clear the intersection first. A procedure to determine this supplemental delay is described in detail in Appendix F of the Highway Capacity Manual 2000. This procedure is also extended to analyze delay over multiple time periods where a residual demand may be carried from one time period to the next. Due to the lack of information on queue formation, a value of zero was used for supplemental delay $\left(\mathrm{d}_{3}\right)$.

\subsubsection{Typical Delay Associated with Sub-optimal Signal Timing}

According to the Institute of Transportation Engineers (ITE), there are about 300,000 traffic signals in the United States. Over 75 percent of these signals could easily be improved by updating equipment or by simply adjusting their timing. The total number of signal-controlled intersections specified in this ITS study is consistent with the total number of signal-controlled intersections estimated based on the HPMS Sample Database. 
According to FHWA's Arterial Management Benefits database, approximately a 15-20 percent reduction in delay can be achieved by signal-timing updates and/or improvements. Up to a 40 percent reduction in delay can be achieved by implementing automated signal control. This study based its estimates of delay due to sub-optimal signal timing on the percentage of delay reduction that can be realized from improving signal timing.

In order to be conservative in estimating delays, the TLC2 study assumed signal timing to be inadequate at 50 percent of signals, rather than the 75 percent estimated by ITE. Furthermore, it was assumed that a 15 percent reduction in delay could be achieved by correcting these signals. This is at the lower end of the range estimated by the FHWA Arterial Management Benefits database.

\subsubsection{Typical Capacity Loss Associated with Sub-optimal Signal Timing}

According to the ITS Deployment Analysis System (IDAS) User's Manual (Cambridge Systematics, Inc., 2001), updated and improved signal timing can increase capacity at signal-controlled intersections by about 8-23 percent. The amount of improvement depends on traffic condition, time since the last signaltiming update, and intersection densities. This study assumed that signal control could be improved on 50 percent of the signals and that a 10 percent increase of intersection capacity could be achieved. Again, the assumptions were intended to be conservative.

\subsection{RESULTS}

\subsubsection{Delay Due to Sub-optimal Signal Control}

Based on HPMS data, this study estimates that, in 1999, the nation's highway system employed signalized control at 306,177 intersections, 106,859 of which were utilized on principal arterials. The delay from sub-optimal signal controls on principal arterial intersections is estimated at 295.8 million vehicle-hours (Table 28). As expected, the lion's share of this delay (over 97 percent) occurred on urban principal arterials since urban streets contain more signalized intersections. In addition, most of this delay (about 61 percent) occurred during off-peak periods. 
Table 28. Capacity reduction \& delay from non-optimal signal timings on principal arterials

\begin{tabular}{|c|c|c|c|c|c|}
\hline Highway type & $\begin{array}{c}\text { Urban } \\
\text { area size* }\end{array}$ & $\begin{array}{c}\text { Peak } \\
\text { period }^{\dagger}\end{array}$ & $\begin{array}{c}\text { Congestion } \\
\text { level }^{\ddagger}\end{array}$ & $\begin{array}{c}\text { Capacity reduction } \\
(1,000 \text { vehs })\end{array}$ & $\begin{array}{c}\text { Delay } \\
(1,000 \text { veh-hrs }) \\
\end{array}$ \\
\hline \multirow{13}{*}{$\begin{array}{l}\text { Urban other } \\
\text { principal arterials }\end{array}$} & \multirow{3}{*}{$\begin{array}{l}\text { Very } \\
\text { large }\end{array}$} & \multirow{2}{*}{ Peak } & Congested & $1,391,680.9$ & $24,118.5$ \\
\hline & & & Not congested & $7,927,766.9$ & $21,739.7$ \\
\hline & & \multicolumn{2}{|c|}{ Off-peak } & $35,413,901.5$ & $67,057.5$ \\
\hline & \multirow{3}{*}{ Large } & \multirow{2}{*}{ Peak } & Congested & $840,607.3$ & $9,650.8$ \\
\hline & & & Not congested & $6,844,530.1$ & $12,758.0$ \\
\hline & & \multicolumn{2}{|c|}{ Off-peak } & $29,203,521.9$ & $34,425.8$ \\
\hline & \multirow{3}{*}{ Medium } & \multirow{2}{*}{ Peak } & Congested & $636,706.0$ & $5,881.4$ \\
\hline & & & Not congested & $2,575,698.6$ & $5,313.4$ \\
\hline & & \multicolumn{2}{|c|}{ Off-peak } & $12,207,137.4$ & $16,985.3$ \\
\hline & \multirow{3}{*}{ Small } & \multirow{2}{*}{ Peak } & Congested & $1,080,626.3$ & $11,234.7$ \\
\hline & & & Not congested & $13,316,900.0$ & $22,734.7$ \\
\hline & & \multicolumn{2}{|c|}{ Off-peak } & $54,710,600.0$ & $55,685.1$ \\
\hline & \multicolumn{3}{|l|}{ Total } & $166,149,676.8$ & $287,584.9$ \\
\hline \multirow{4}{*}{\multicolumn{2}{|c|}{ Rural other principal arterials }} & \multirow{2}{*}{ Peak } & Congested & $92,435.2$ & 445.2 \\
\hline & & & Not congested & $1,304,590.6$ & $2,210.8$ \\
\hline & & \multicolumn{2}{|c|}{ Off-peak } & $5,308,698.1$ & $5,584.6$ \\
\hline & & \multicolumn{2}{|l|}{ Total } & $6,705,723.9$ & $8,240.6$ \\
\hline \multicolumn{4}{|l|}{ Total } & $172,855,400.7$ & $295,825.5$ \\
\hline
\end{tabular}

* Urban area size categories are based on population: very large - more than 3 million; large - 1 to 3 million; medium 0.5 to 1 million; small - less than 0.5 million.

${ }^{\dagger}$ Peak periods: 6:00 am to 9:30 am and 3:30 pm to 7:00 pm Monday through Friday; all others considered non-peak.

*A roadway section is considered congested during the peak periods if its Volume/Service Flow Ratio (V/SF) is greater than $95 \%$. 


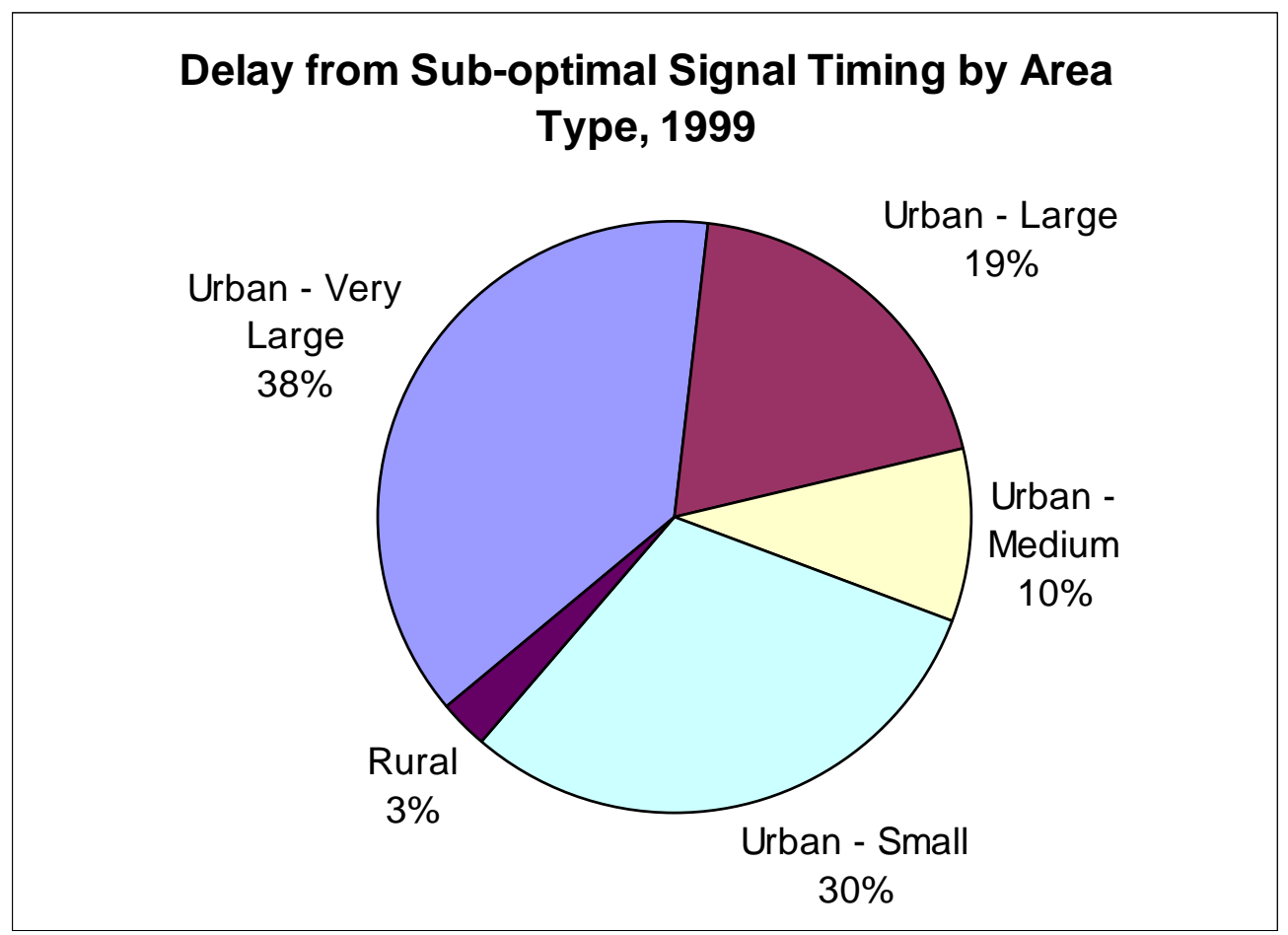

Fig. 24. Delay from sub-optimal signal timing was greatest in very large urban areas.

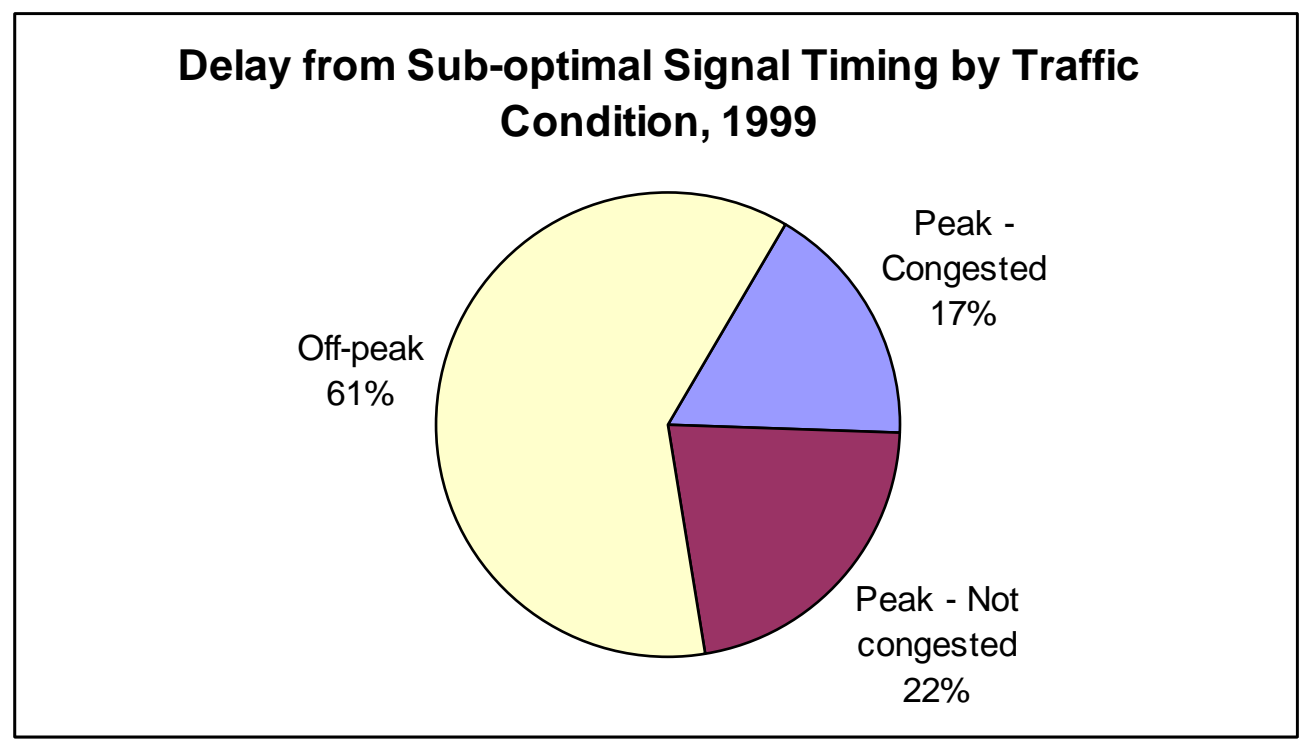

Fig. 25. Most delay from sub-optimal signal timing occurred during off-peak periods.

\subsubsection{Capacity Losses Due to Sub-optimal Signal Control}

The TLC2 study estimates that capacity loss on principal arterials due to sub-optimal signal control was nearly 173 billion vehicles in 1999 (Table 29). Again, about 97 percent of this reduction occurs on urban principal arterials. Capacity losses estimated for signal timing are much larger than capacity losses associated with crashes and vehicle breakdowns. This is because capacity losses at signal-controlled 
intersections occur 24 hours a day 365 days a year, regardless of demand. Therefore, these reductions occur but have less actual impact. The durations for the capacity losses associated with crashes and vehicle breakdowns, on the other hand, are much shorter, and they often coincide with high traffic volumes.

Table 29. Capacity loss on principal arterials due to sub-optimal signal control, 1999 (million vehicles)

\begin{tabular}{lrrrrr}
\hline \multirow{2}{*}{ Arterial type } & \multicolumn{5}{c}{ Signal control type } \\
\cline { 2 - 6 } & Fixed time & Actuated & Coordinated & Unknown & \multicolumn{1}{c}{ Total } \\
\hline Rural principal arterials & 675 & 1,071 & 104 & 4,857 & 6,706 \\
Urban principal arterials & 39,978 & 77,296 & 48,873 & 5 & 166,150 \\
All principal arterials & 40,653 & 78,367 & 48,977 & 4,861 & 172,856 \\
\hline
\end{tabular}

\subsection{RELIABILITY}

\subsubsection{Methodology}

The TLC2 study uses methodologies suggested by the Highway Capacity Manual to calculate delay at signal-controlled intersections. These methods are used by most traffic engineers within the United States and are well established. The delay estimates generated by these methodologies are qualified as having a high degree of confidence.

\subsubsection{Data \& Key Assumptions}

The signal and traffic operation information used to estimate delay at signal-controlled intersections is based on "Sample" data from HPMS. This HPMS data is used extensively in the analysis of highway system condition, performance, and investment needs that make up the biennial Condition and Performance Reports to Congress. This data is accorded a high level of confidence.

Signal parameters that could not be inferred from HPMS data were based on values suggested by the Highway Capacity Manual were used. These parameters are accorded a high level of confidence.

The Institute of Transportation Engineers (ITE) estimates that signal timing is inadequate at 75 percent of all intersections in the U.S. In order to be conservative, TLC2 assumes 50 percent of the national's signal controlled intersections have inadequate signal timing. This is qualified as having a medium degree of confidence.

FHWA's Arterial Management Benefits database indicates that approximately 15-20 percent delay reductions can be achieved by signal-timing updates and/or improvements. This information is based on numerous recent studies across United States. In order to be conservative, TLC2 assumes a 15 percent reduction in delay. This is qualified as having a high degree of confidence. 


\section{RAILROAD CROSSINGS}

\subsection{METHODOLOGY}

Railroad-highway at-grade crossings cause delays when vehicles must stop to yield the right of way to trains. This temporarily reduces the highway capacity to zero for a brief period of time, depending on the speed and the length of the train. Estimates of delays at railroad-highway crossings for TLC2 were based on data produced by a study conducted by the Federal Railroad Administration (FRA) Office of Policy and Program Development (unpublished data). The FRA delay estimates were based on data compiled in the FRA Highway-Rail Crossing Database (U.S. DOT/FRA 2003) and methodologies used in the Highway-Rail Grade Crossing Evaluation Software, or GradeDec 2000 (U.S. DOT/FRA 2002).

In general, the delay estimates are based on queuing theory. Highway vehicle delay depends on the highway vehicle arrival rate (highway traffic volume), frequency of the crossing blockage (railroad traffic volume), and the blockage time (train speed and length). The estimates produced by FRA are for all highway-rail at-grade crossings. Since delays on minor arterials, collectors, and local roads are beyond the scope of TLC2, only those delays incurred on principal arterials were tabulated - Interstates do not have at-grade crossings.

\subsection{RESULTS}

This study estimates that at-grade highway-railroad crossings caused about 2.9 million vehicle-hours of delay on principal arterials in 1999. Most of this delay (91 percent) occurred in urban areas, and most of the urban delay occurred during off-peak hours. Crossings in small urban areas accounted for nearly half (48 percent) of the delay, followed by those in very large urban areas (23 percent). Delay at crossings in medium and large urban areas, combined, only accounted for about 20 percent. Crossings in rural areas accounted for 9 percent of delay. Overall, 96 percent of all delay occurred during off-peak or noncongested peak hours; only 4 percent occurred during congested, peak-hour traffic conditions. 


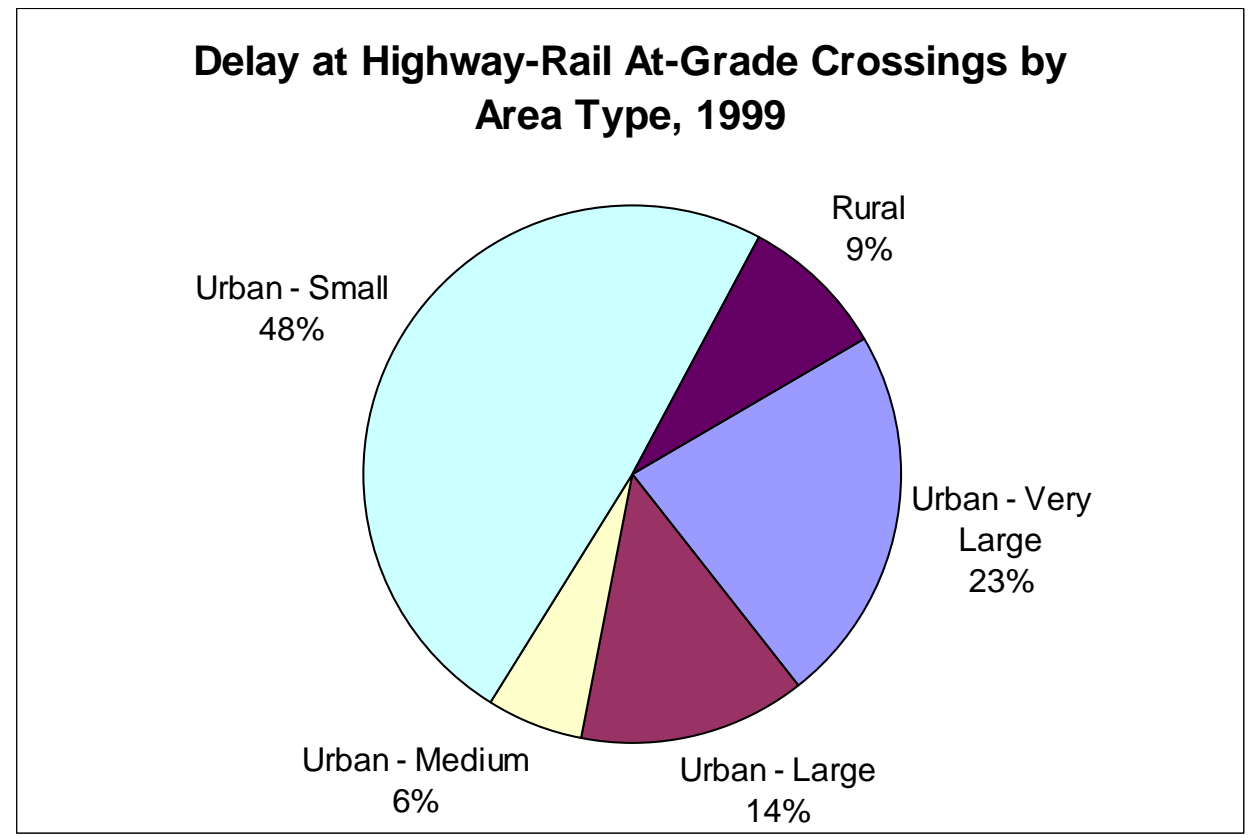

Fig. 26. Nearly half of the delay from highway-rail at-grade crossings was experienced in small urban areas.

Table 30. Delay from at-grade highway-railroad crossings, 1999

\begin{tabular}{|c|c|c|c|c|}
\hline Highway type & $\begin{array}{c}\text { Urban area } \\
\text { size* }\end{array}$ & Peak period ${ }^{\dagger}$ & $\begin{array}{c}\text { Congestion } \\
\text { level }^{\ddagger}\end{array}$ & $\begin{array}{c}\text { Delay } \\
(1,000 \text { veh-hrs })\end{array}$ \\
\hline \multirow{13}{*}{$\begin{array}{c}\text { Urban other } \\
\text { principal arterials }\end{array}$} & \multirow{3}{*}{ Very large } & \multirow{2}{*}{ Peak } & Congested & 41.0 \\
\hline & & & Not congested & 173.7 \\
\hline & & \multicolumn{2}{|l|}{ Off-peak } & 457.4 \\
\hline & \multirow{3}{*}{ Large } & \multirow{2}{*}{ Peak } & Congested & 20.8 \\
\hline & & & Not congested & 107.6 \\
\hline & & \multicolumn{2}{|l|}{ Off-peak } & 273.7 \\
\hline & \multirow{3}{*}{ Medium } & \multirow{2}{*}{ Peak } & Congested & 7.9 \\
\hline & & & Not congested & 47.0 \\
\hline & & \multicolumn{2}{|l|}{ Off-peak } & 116.9 \\
\hline & \multirow{3}{*}{ Small } & \multirow{2}{*}{ Peak } & Congested & 46.4 \\
\hline & & & Not congested & 414.1 \\
\hline & & \multicolumn{2}{|l|}{ Off-peak } & 981.1 \\
\hline & \multicolumn{3}{|l|}{ Total } & $2,687.6$ \\
\hline \multirow{4}{*}{\multicolumn{2}{|c|}{ Rural other principal arterials }} & \multirow{2}{*}{ Peak } & Congested & 3.4 \\
\hline & & & Not congested & 71.8 \\
\hline & & \multicolumn{2}{|l|}{ Off-peak } & 184.4 \\
\hline & & \multicolumn{2}{|l|}{ Total } & 259.6 \\
\hline \multicolumn{4}{|l|}{ Total } & $2,947.2$ \\
\hline
\end{tabular}

* Urban area size categories are based on population: very large - more than 3 million; large - 1 to 3 million; medium 0.5 to 1 million; small - less than 0.5 million.

${ }^{\dagger}$ Peak periods: 6:00 am to 9:30 am and 3:30 pm to 7:00 pm Monday through Friday; all others considered non-peak.

*A roadway section is considered congested during the peak periods if its Volume/Service Flow Ratio (V/SF) is greater than $95 \%$. 


\subsection{RELIABILITY}

The estimates of delay due to at-grade highway-railroad crossings are taken directly from a database prepared by a Federal Railroad Administration study. The results of the study have been published in the 2002 Status of the Nation's Highways, Bridges, and Transit: Conditions \& Performance, Report to Congress, but the baseline delay estimates used by TLC are not published in the Conditions and Performance Report. Potential improvements in delay under different investment scenarios are presented in that report. The baseline delay information used for TLC2 is qualified as having a medium degree of confidence. 


\section{TOLL FACILITIES}

\subsection{METHODOLOGY}

The delay caused by toll facilities is an ever-increasing concern to the transportation community. Today, over 115 authorities operate nearly 250 facilities in 31 states. These facilities comprise more than 900 toll plazas. In the U.S, toll roads account for 4,800 miles of both rural and urban roads. Drivers passing through these toll plazas perform over five billion transactions, transactions that result in approximately 5.7 billion dollars of revenue annually.

Unlike other TLC impacts, the method used to determine delay from toll facilities is not directly based on estimating capacity reduction and modeling vehicle queue build-up and dissipation. This is because the total capacity of a toll facility is difficult to determine using available data. Although information on the number of toll lanes is generally available, all lanes are not always open. Without knowing the number of lanes open to traffic, it is difficult to determine capacity. Therefore, an alternative method was devised comprised of the following steps:

Step 1. Identify all toll collection facilities and their locations

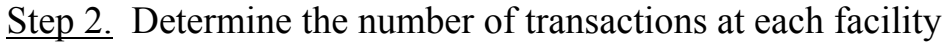

Step 3. Determine the type of toll collection used at each facility

Step 4. Estimate the total delay at each facility based on the number of transactions and the toll collection types used

Step 5. Sum the delay for all collection facilities

These steps are described in the sections that follow.

\subsubsection{Identifying Toll Facilities and Their Locations}

The first step in estimating toll facility delay was to identify all toll facilities on freeways and principal arterials. The primary data source for this information was Toll Facilities in the United States: Bridges Roads - Tunnels - Ferries published by the Federal Highway Administration's Office of Highway Policy Information (OHPI) (FHWA 2003). This report contains selected information on all toll facilities in the United States, such as name, location, financing/operating authority, toll direction, mileage, and type of toll collection. The information is based on a survey of facilities in operation, financed, or under construction as of January 1, 2003. TLC2 used the toll facilities listing in this report as its "master list" of toll facilities. Another document entitled "United States Toll Facilities" by Traffic Technologies, Inc. was used as a supplement to the OHPI report. ${ }^{8}$

\subsubsection{Determining the Number of Transactions at Each Facility}

Once all toll facilities on freeways and principal arterials were identified, the next step was to determine the number of transactions performed at each. The most detailed and reliable source of this information

\footnotetext{
${ }^{8}$ The United States Toll Facilities document was taken from a web site that is no longer operational.
} 
are the web pages and annual reports of the toll facility operating authorities. However, some toll facility operating authorities regard the toll transaction information as business sensitive. In these instances, transaction information can only be obtained from the authority's legal department using the Freedom of Information Act (FOIA). For this study, transaction information was obtained for about 83 percent of the facilities within the study's scope.

For instances where transaction information from the toll authority was not available or could not be obtained, Annual Average Daily Traffic (AADT) information from HPMS was used as a surrogate for the number of daily transactions. As a final option, annual total toll transaction information from the document "United States Toll Facilities" was used if information was not available from the above sources.

\subsubsection{Determining Toll Facility Type}

In order to properly assign delay to a given facility, the methods used to determine toll rates and collect tolls must be considered.

\section{Open vs. Closed Systems}

For long toll roads, two common methods are used to determine the toll rate. The first is the open system. In the open system, there are many toll facilities along the main-line toll road, and drivers pay a toll at each facility they encounter. For this type of facility, each transaction represents one vehicle paying toll at one facility. The other method of toll collection is called the closed system. In a closed system, typically used with ticketed toll facilities, the driver stops and receives a ticket stamped with the location of the entrance to the toll facility. He/she stops again upon exiting the facility and pays the toll, which is based on the point of entry and point of exit along the facility route. Therefore, in the closed system, each transaction represents one vehicle stopping twice.

\section{Toll Collection Method}

Three types of toll collection are used at modern toll facilities:

Manual, or manned, toll facilities. The most common method is the manual tollbooth, where drivers pay the toll to an attendant who then raises a gate to permit the vehicle to pass. While this method is the most common, it causes the most delay of any of the facilities (the cost to maintain a toll collector at each booth also makes it expensive).

Coin-Basket Facility. The coin-basket facility uses an unmanned booth where drivers stop at the tollbooth and toss the exact change in coins into a basket. The machine determines whether the correct amount of toll has been paid and, if so, raises a gate to permit the vehicle to pass. This system causes less delay than a wholly manual system. However, drivers must have exact change and must "hit" the basket with it. Otherwise, drivers can become stuck in the queue. Though common, these systems have begun to decline in popularity in recent years.

Electronic Toll Collection (ETC) systems. In the ETC system, drivers subscribe to a service and are given a transponder. Toll facilities are outfitted to detect the transponder and subtract the toll money from the driver's account when his/her vehicle passes the booth. This system creates the least amount of delay, since drivers must merely slow down, rather than stop, for payment.

Like the transaction data, information on toll collection types was obtained from the annual reports of facility operating authorities or from other information on their web sites. Toll collection method data was 
available for about 83 percent of facilities. For the remaining facilities, these shares were based on the average shares for the 83 percent for which data was available.

\subsubsection{Estimating Delay at Each Facility}

Delay from toll collection can be divided into three types: (1) "process" or "service" delay resulting from the time it takes to make the fee transaction, (2) "slow down" delay from slowing down and speeding up at the facility, and (3) queue delay in cases where a line of vehicles is waiting in line.

For this study, process time for manual and coin-basket facilities was assumed to be 6 seconds. This is loosely based on data from a report entitled Electronic Toll and Traffic Management (ETTM) Systems - A Synthesis of Highway Practice (NCHRP 1993). According to that study, manual collections facilities can process about 350 to 450 vehicles per hour per lane, while coin-basket facilities can process about 500 to 600 . The 6-second process time corresponds with a capacity of 600 vehicles per hour per lane. It was assumed that ETC facilities can process a transaction in half as much time, although it may actually take even less.

Slow down delay for manual and coin-basket facilities was assumed to be 10 seconds. This was loosely based on the fact that it takes most vehicles from 5 to 12 seconds to reach a speed of 60 miles per hour from a complete stop - although this obviously varies due to driver behavior and vehicle performance. It was assumed that a similar amount of time was required to come to a complete stop. The study also assumed that slow down time for ETC facilities is about half of that for manual and coin-basket facilities ( 5 seconds) since vehicles need only slow down while driving through the facility, rather than coming to a complete stop.

For this study, queue delay was assumed to take place only at manual collection facilities during peak hours. Data collected by the New York City Metropolitan Transportation Authority (MTA) shows that peak-hour queue time at toll bridges and tunnels under their authority ranged from 18 to 29 seconds in 2002 and the first quarter of 2003. This study, therefore, assumed that average queue time at manual facilities during peak hours was 20 seconds. It was also assumed that no queue delay is experienced during non-peak hours at these facilities. Several studies in the literature show that ETC facilities reduce delay to the point that no, or almost no, queue delay was experienced (Wilbur Smith \& Assoc. 2001; Vollmer Associated LLP 2000; Saka 2002). Therefore, it is assumed that queue delay for ETC transactions is zero.

The per-vehicle delay assumptions used in this study are summarized in the table below.

Table 31. Per-vehicle delay assumptions by toll collection and delay type

\begin{tabular}{l|c|c}
\hline \multirow{2}{*}{ Delay type } & \multicolumn{2}{|c}{ Toll collection type } \\
\cline { 2 - 3 } & Manual & Electronic \\
\hline Process & 6 seconds & 3 seconds \\
\hline Slow down & 10 seconds & 5 seconds \\
\hline Queue & 20 seconds & 0 seconds \\
\hline
\end{tabular}

In this study, total delay at a given toll facility was calculated as the sum of the delay for (1) the manual collection lanes during peak hours, (2) manual collection lanes during off-peak hours, and (3) electronic toll collection lanes during peak and off-peak hours - delay for coin-basket lanes is calculated the same as for manual lanes. The following equation was used: 


$$
\begin{aligned}
& \text { Delay }=\left(\text { Delay }_{M P}+\text { Delay }_{M S}+\text { Delay }_{M Q}\right) \times \text { Transactions }_{\text {Total }} \times\left(\frac{\text { Hrs }_{\text {Peak }}}{\text { Hrs }_{\text {Total }}}\right) \\
& +\left(\text { Delay }_{M P}+\text { Delay }_{M S}\right) \times \text { Transactions }_{\text {Total }} \times\left(1-\frac{\text { Hrs }_{\text {Peak }}}{\text { Hrs }_{\text {Total }}}\right) \times\left(1-\frac{\text { Lanes }_{\text {ETC }}}{\text { Lanes }_{\text {Total }}}\right) \\
& +\left(\text { Delay }_{E P}+\text { Delay }_{E S}\right) \times \text { Transactions }_{\text {Total }} \times\left(\frac{\text { Lanes }_{\text {ETC }}}{\text { Lanes }_{\text {Total }}}\right)
\end{aligned}
$$

where

$$
\begin{aligned}
& \text { Delay }_{\mathrm{MP}}=\text { average process delay per vehicle for manual collection lanes } \\
& \text { Delay }_{\mathrm{MS}}=\text { average slowdown delay per vehicle for manual collection lanes } \\
& \text { Delay }_{\mathrm{MQ}}=\text { average queue delay per vehicle for manual collection lanes } \\
& \text { Delay }_{\mathrm{EP}}=\text { average process delay per vehicle for ETC lanes } \\
& \text { Delay } \\
& \text { Trans }_{\mathrm{ES}}=\text { average slowdown delay per vehicle for ETC lanes } \\
& \mathrm{Hrs}_{\text {Peak }}=\text { number of hours a day a facility is closest to its maximum capacity } \\
& \mathrm{Hrs}_{\mathrm{Total}}=\text { total number of hours a day the facility is open } \\
& \text { Lanes }_{\mathrm{ETC}}=\text { number of lanes at a given facility that utilize an ETC system } \\
& \text { Lanes }_{\mathrm{Total}}=\text { total number of toll collection lanes at the facility (both manual and ETC) }
\end{aligned}
$$

\begin{tabular}{|c|c|c|c|c|c|c|}
\hline \multirow{2}{*}{$\begin{array}{c}\text { Structure } \\
\text { type }\end{array}$} & \multirow{2}{*}{$\begin{array}{l}\text { Area } \\
\text { type }\end{array}$} & \multirow[b]{2}{*}{ Highway type } & \multicolumn{3}{|c|}{ Delay $(1,000$ veh-hrs) } & \multirow{2}{*}{$\begin{array}{l}\text { Delay/veh } \\
\text { (seconds) }\end{array}$} \\
\hline & & & Electronic & Manual* & Total & \\
\hline \multirow{6}{*}{$\begin{array}{l}\text { Bridges } \\
\text { and } \\
\text { tunnels }\end{array}$} & \multirow[t]{3}{*}{ Urban } & Interstate & 487.4 & $1,619.7$ & $2,107.1$ & 9.4 \\
\hline & & Freeways & 266.5 & $1,293.7$ & $1,560.2$ & 8.1 \\
\hline & & Principal arterials & 109.2 & 840.9 & 950.1 & 13.8 \\
\hline & \multirow[t]{2}{*}{ Rural } & Interstate & 21.0 & 254.7 & 275.6 & 11.7 \\
\hline & & Principal arterials & 16.7 & 204.2 & 220.9 & 13.9 \\
\hline & All & All & 900.7 & $4,213.2$ & $5,113.8$ & 11.4 \\
\hline \multirow[t]{3}{*}{ Toll roads } & \multirow{3}{*}{$\begin{array}{l}\text { Urban \& } \\
\text { rural }\end{array}$} & Interstate & 942.2 & $4,639.2$ & $5,581.3$ & 14.6 \\
\hline & & $\begin{array}{l}\text { Freeways \& } \\
\text { principal arterials }\end{array}$ & $2,913.4$ & $7,431.7$ & $10,345.1$ & 12.2 \\
\hline & & All & $3,855.6$ & $12,070.9$ & $15,926.5$ & 13.4 \\
\hline \multicolumn{3}{|l|}{ Total $^{\dagger}$} & $4,756.2$ & $16,284.0$ & $21,040.3$ & 11.9 \\
\hline
\end{tabular}

\subsection{RESULTS}

The study estimates that 21 million vehicle-hours of delay were caused by toll collection facilities in 1999. The average delay per transaction was 11.9 seconds. This resulted in 33.6 person-hours of delay. Nearly three-quarters of this delay, was incurred at manual and coin-basket (i.e., non-ETC) collection facilities.

Table 32. Delay from toll collection facilities on freeways \& principal arterials, 1999

* Includes coin-basket facilities.

Some totals may not sum due to rounding. 


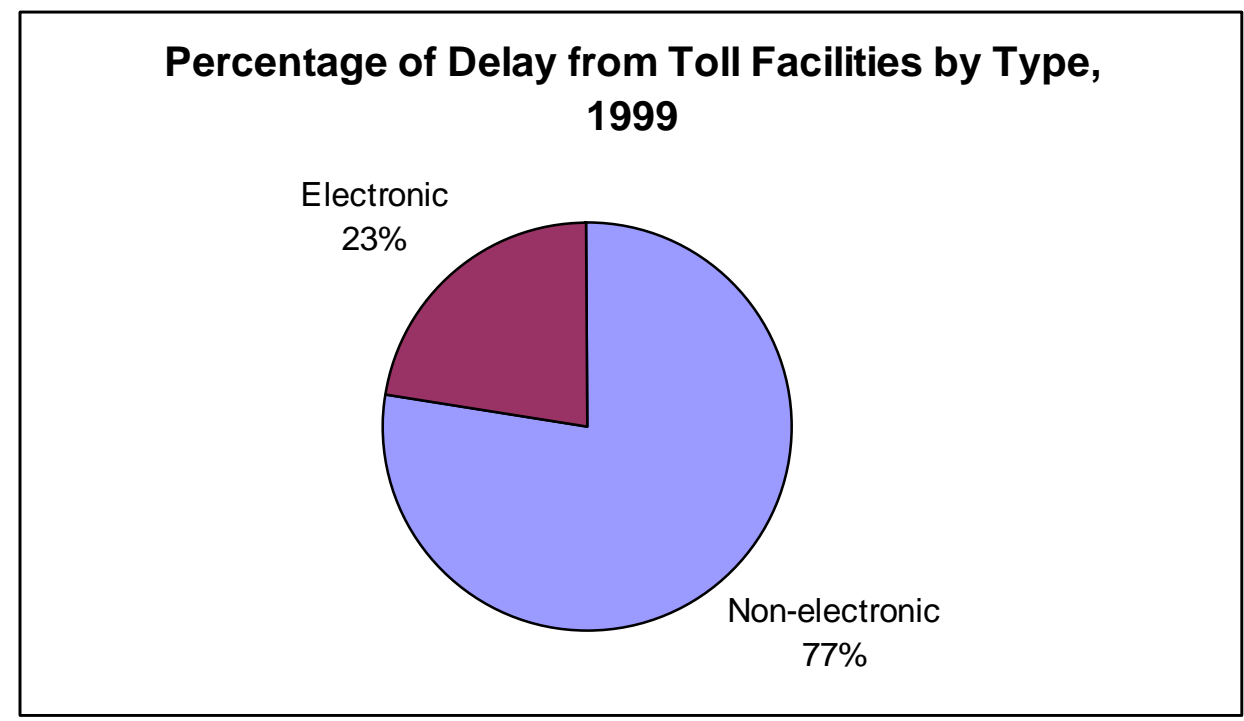

Fig. 27. Over three quarters of the delay from toll facilities in 1999 occurred in lanes using non-electronic collection methods.

\subsection{RELIABILITY}

\subsubsection{Methodology}

The methodology for estimating toll facility delay is simple and straightforward. It is accorded a high level of confidence.

\subsubsection{Data \& Key Assumptions}

The service rates for both manual and electronic toll collection system are based on many recently published studies.

Slowdown and speed resumption delays are based on acceleration rates for all passenger vehicles from recently published reports. Therefore, this information is qualified as having a high degree of confidence.

Assumptions regarding queue formation and queue delays are based solely on a study of toll facilities operated by the New York City Metropolitan Transportation Authority; there is little information on queue delay at other toll facilities. Therefore, this information is qualified as having a low degree of confidence.

The total number of transactions and the percent of transactions processed by ETC are primarily based on toll operator annual reports. Data were available for $83 \%$ of toll facilities. Other transaction information is based either on traffic volume information or older ( 1 to 2 year-old) transaction information. This information is accorded a medium to high degree of confidence. 


\section{COMMERCIAL TRUCK PICKUP AND DELIVERY (PUD) ACTIVITIES IN URBAN AREAS}

\subsection{METHODOLOGY}

Pickup and delivery (PUD) activities of large trucks in urban areas can cause highway capacity reductions and traffic delays when these vehicles are parked illegally, partially or fully blocking a lane of traffic. Commercial drivers park their vehicles illegally for several reasons, including lack of parking near the PUD point or a desire to park their vehicle where it can be easily seen to prevent theft or vandalism.

The method used to estimate the delay from commercial truck PUD activities varies somewhat from the methods used for other capacity-reducing phenomena in this study. Rather than using a Monte Carlo simulation method to place lane-blocking PUD events on highway links at a specific time and location and calculating the resulting capacity loss and delay, this study estimates a weighted average delay value for lane-blocking PUD activities on each link, which are summed for each urban area and multiplied by the estimated number of lane-blocking PUD activities for that area. The steps used to estimate delay from commercial truck PUD activities can be summarized as follows:

Step 1. All urban arterial links were identified and assigned to an FHWA urbanized area using FHWA’s Highway Performance Monitoring System (HPMS) data.

Step 2. The weighted average capacity reduction and delay resulting from a single PUD activity on each urban link was determined.

Step 3. The average delay for a single PUD activity occurring in each urban area was estimated by averaging across all links and weighting by the length of each link.

Step 4. The annual number of PUD activities occurring in each urban area was estimated based on employment statistics for each land use type (i.e., commercial, industrial, and office) and trip generation factors that apply to each land use type-PUD activities were assumed to only occur within ZIP codes where the total annual salary was in excess of $\$ 600$ million per square mile.

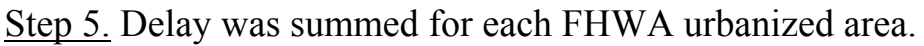

These steps are described in more detail in the sections that follow.

\subsubsection{Identifying Arterial Links and Assigning Them to the Appropriate FHWA Urbanized Area}

HPMS data was used to identify all links classified as urban arterials. This included links designated as functional system code 14 (other principal arterials), 16 (minor arterials), and 17 (collectors) in HPMS. These links were also assigned to the FHWA urbanized area in which they were located.

\subsubsection{Estimating Weighted Average Capacity Reduction and Delay per PUD Event on Each Link for Each Land Use Type}

For each urban principal arterial, the average capacity reduction and delay that would result from a laneblocking PUD was estimated for each of three land use types (commercial, office, and industrial). A method similar to the one used to estimate capacity reduction and delay for crashes, breakdowns, and work zones was used, with some minor changes. 
First, PUD events were not broken down into time intervals with different capacity-reducing characteristics; each event was modeled as a single interval. The dwell time for each event was assumed to be 15 minutes. This was based on observed dwell times reported in studies by Aherns et al. (1977) and Habib (1980). Aherns observed an average dwell time of 17.4 minutes per delivery for all stores participating in its survey. The Habib study also reported an average dwell time of 19.5 minutes for PUD trucks legally parked at curb-side (5,046 observations), 13.8 minutes for trucks curb-parked illegally (1,697 observations), and 11.5 minutes for those double-parking in a moving lane (1,398 observations).

It was assumed that an illegally parked truck will block one lane of traffic - the one closest to the curb. If this event occurs on a link that has only one lane in that direction, it was assumed that traffic will go around the parked vehicle by partially crossing over into the oncoming lane.

For each link, the delay was averaged across all applicable hours of the day (6:00 am to 5:00 pm) and days of the week (Monday through Friday), weighting each time slice by the probability of a PUD activity taking place. An hourly PUD arrival distribution observed by Habib was used to weight delay by time of day for each land use type. The arrival distribution used to weight delay by day of the week was taken from Aherns et al. These distributions are presented in the tables below.

Table 33. Hourly PUD arrival distribution percentages

\begin{tabular}{lrcc}
\hline \multirow{2}{*}{ Time of day } & \multicolumn{3}{c}{ Land use type } \\
\cline { 2 - 4 } & Commercial & Office & Industrial \\
\hline 6:00 - 7:00 a.m. & 1.0 & 0.1 & 0.2 \\
7:00 - 8:00 a.m. & 2.8 & 1.4 & 2.4 \\
8:00 - 9:00 a.m. & 7.7 & 9.6 & 14.0 \\
9:00 - 10:00 a.m. & 16.5 & 14.4 & 15.4 \\
10:00 - 11:00 a.m. & 18.1 & 16.6 & 18.1 \\
11:00 - 12:00 p.m. & 14.6 & 13.4 & 12.4 \\
12:00 - 1:00 p.m. & 11.0 & 11.0 & 8.6 \\
1:00 - 2:00 p.m. & 10.6 & 11.4 & 10.8 \\
2:00 - 3:00 p.m. & 10.4 & 11.9 & 10.0 \\
3:00 - 4:00 p.m. & 7.1 & 9.9 & 7.4 \\
4:00 - 5:00 p.m. & 0.2 & 0.3 & 0.5 \\
\hline
\end{tabular}

Table 34. PUD arrival percentages by day of week

\begin{tabular}{l|c}
\hline Day of week & $\%$ of PUD events \\
\hline Monday & 16.6 \\
\hline Tuesday & 16.4 \\
\hline Wednesday & 18.6 \\
\hline Thursday & 21.8 \\
\hline Friday & 26.6 \\
\hline
\end{tabular}

The equation used to estimate weighted average delay for each link for each land use type is provided below. 


$$
\overline{D_{l_{f=14}, q}}=\sum_{d=1 \text { to } 5} \sum_{t=6 \text { to } 17} D_{l, t, d} \times \text { HFactor }_{t, q} \times \text { DFactor }_{d}
$$

where

$$
\mathrm{D}=\text { delay }
$$

HFactor $=$ probability of PUD activity by hour of the day and land use type

DFactor $=$ probability of PUD activity by day of the week

$1=$ link

$\mathrm{f}=$ FHWA functional classification $(14=$ other principal arterial, 16=minor arterial, $17=$ collector $)$

$\mathrm{q}=$ land use type (commercial, office, industrial)

$\mathrm{d}=$ weekday, Monday through Friday (i.e., 1 to 5 )

$\mathrm{t}=$ time of the day from 6:00 am to 5:00 pm (i.e., 6 to 17)

\subsubsection{Estimating Weighted Average Delay per PUD Event for Each Urban Area for Each Land Use Type}

Once the average delay per link was determined for each land use type, the average delay across all principal arterial links in each urban area for each land use type was estimated. Average delay was estimated by multiplying the delay for each urban principal arterial link by the percentage of total arterial mileage categorized as other principal arterial (functional class 14). This was calculated using the following equation.

$$
\overline{D_{u, q}}=\sum_{l_{f=14}=1 t_{0}}\left(D_{l_{f}} \times \frac{L_{l_{f}=14}, u, q}{\sum_{f=14,16,17} \sum_{l=1 \text { to } n_{f}} L_{l_{f}}}\right)
$$

where

$$
\begin{aligned}
& \text { D = delay } \\
& \mathrm{u}=\text { FHWA urbanized area } \\
& \mathrm{q}=\text { land use type (commercial, office, } \text { industrial) } \\
& \mathrm{l}=\text { link } \\
& \mathrm{f}=\text { FHWA functional classification }(14=\text { other principal arterial, } 16=\text { minor arterial, } 17=\text { collector }) \\
& \mathrm{L}=\text { length }
\end{aligned}
$$

\subsubsection{Estimating Annual Delay for Each Urban Area Based on Number of PUD Events}

The next step was to estimate the annual delay for each urban area. Annual delay was estimated by multiplying the average delay per lane-blocking PUD event for each urbanized area by the annual number of lane-blocking events for that area. The number of PUD events (including those that do not block lanes) 
was estimated by applying PUD trip generation rates by the number of people employed within in each land use type. To estimate the number of PUD events that blocked lanes, it was assumed that $20 \%$ of all PUD events involve illegal parking that blocks a lane. This assumption was based on the Habib study that reported that $20-25 \%$ of PUD vehicles parked illegally in a curb-side moving lane. The equation used to estimate annual delay for each urbanized area is given below.

$$
D_{u}=\sum_{q}\left(D_{u, q} \times E_{u, q} \times R_{q} \times B \times W\right)
$$

where

$$
\begin{aligned}
& D=\text { delay } \\
& E=\text { number of employees } \\
& R=\text { trip rate (number of PUD activities generated per number of employees) } \\
& B=\text { percent of PUD activities that block lanes due to illegal parking } \\
& W=\text { work days in a year (260) } \\
& \mathrm{u}=\text { FHWA urbanized area ( } 400 \text { areas designated as urban) } \\
& \mathrm{q}=\text { land use type (commercial, office, industrial) }
\end{aligned}
$$

The rate for PUD events was based on truck trip generation data from the National Cooperative Highway Research Program (NHCRP) publication Truck Trip Generation Data: A Synthesis of Highway Practice, NCHRP Synthesis 298 (Fischer and Han 2001). The NHCRP report contains tables that provide trip rates per employee by type of land use. The NHCRP trip rates used in the TLC2 study were taken from a 1993 study in Tampa, Florida, by Gannett Fleming, Inc. (Table 35). For each land use type, the average rate for all truck types was used.

Table 35. Truck trip rates (12-hour) per employee

\begin{tabular}{llccc}
\hline \multirow{2}{*}{ Land use } & Truck type & \multicolumn{3}{c}{ Trip rate } \\
\cline { 3 - 5 } Commercial & Light & 0.071 & 0.178 & 0.432 \\
& Heavy & 0.009 & 0.047 & 0.075 \\
& All & 0.080 & $\mathbf{0 . 2 2 5}$ & 0.507 \\
\hline Office & Light & 0.019 & 0.038 & 0.075 \\
& Heavy & 0.003 & 0.009 & 0.015 \\
& All & 0.022 & $\mathbf{0 . 0 4 7}$ & 0.090 \\
\hline Industrial & Light & 0.077 & 0.285 & 0.718 \\
& Heavy & 0.039 & 0.164 & 0.335 \\
& All & 0.116 & $\mathbf{0 . 4 4 9}$ & 1.053 \\
\hline
\end{tabular}

* Based on a survey conducted in Tampa, FL

Employment estimates for each land use type in each urban area were based on two sources: U.S. Census Bureau ZIP Code Business Patterns data and U.S. Department of Labor (U.S. DOL) Bureau of Labor Statistics (BLS) Covered Employment and Wages (CEW) Program data. 
The Census ZIP Code Business Patterns dataset provides business data summarized for nine employmentsize classes by hundreds of North American Industry Classification System (NAICS) codes and about 40,000 ZIP codes nationwide. The database is geo-coded based on the ZIP Code Boundary \& Inventory Files developed by Geographic Data Technology and contains location data for ZIP centroids. However, data on self-employed persons, domestic service workers, railroad employees, agricultural production workers, most government employees, and employees on ocean-borne vessels or in foreign countries are beyond the scope of the dataset.

Therefore, in order to include trips generated by government sites, the BLS data was used to supplement the Census data. The BLS data includes statistics on the number of establishments, monthly employment, and quarterly wages, by NAICS industry, by county, by ownership sector, for the entire United States. County-based government employment statistics were extracted from this dataset, and it was assumed that the land use type for all such establishments was "office."

The Census and BLS employment data were mapped to FHWA urbanized areas using location data in these data sets along with the Bureau of Transportation Statistics (BTS) Federal-aid Urbanized Area Boundaries database, a geographic database of Federal-aid boundaries for urban areas with a population greater than 50,000. The database includes boundaries for urban areas in all 50 states, the District of Columbia, and Puerto Rico. The Census ZIP-code-based dataset includes location information on the centroid of each ZIP. For each urbanized area, employment for each ZIP whose centroid lay within the urbanized area boundary was added to the total for that area.

A different method was used for mapping county-based BLS data to each urbanized area. The percentage of each county's land area that lay within the urbanized area boundary was multiplied by the total employment for that county. The BLS and Census employment estimates were then summed for each urbanized area.

It was assumed that most capacity loss and delays from PUD activities would occur along roadways in central business districts where buildings are close together and parking is limited. However, within the time and funding constraints of this study, it would be quite difficult to identify these locations.

Therefore, as a surrogate indicator for these conditions, capacity reduction was only assumed to occur on roadways within ZIP codes where the total annual salary of employees working within the ZIP code was $\$ 600$ million per square mile - this data is included in the Census ZIP Code Business Patterns dataset.

Since the truck trip generation factors are for daily trip generation, the delay had to be multiplied by the number of workdays in a year (260) in order to generate annual totals.

The final step was to sum capacity reduction and delay for all urban areas by urban area size category.

\subsection{RESULTS}

The study estimates that, in 1999, PUD activities on urban principal arterials caused a capacity reduction of about 117 million vehicles, resulting in approximately 947 thousand vehicle-hours of delay. Most of this delay (nearly 90 percent) occurred in very large urban areas. Nine (9) percent of the total delay occurred in large urban areas, with medium and small urban areas accounting for 0.1 and 1 percent, respectively. Most of the delay (67 percent) occurred during off-peak hours. 
Table 36. Capacity reduction and delay from PUD events, 1999

\begin{tabular}{|c|c|c|c|c|}
\hline $\begin{array}{l}\text { Urban area } \\
\text { size* }\end{array}$ & $\begin{array}{l}\text { Peak } \\
\text { period }^{\dagger}\end{array}$ & Congestion level ${ }^{*}$ & $\begin{array}{c}\text { Capacity reduction } \\
(1,000 \text { vehicles })\end{array}$ & $\begin{array}{c}\text { Delay } \\
(1,000 \text { veh-hrs })\end{array}$ \\
\hline \multirow{3}{*}{ Very large } & \multirow{2}{*}{ Peak } & Congested & $3,322.8$ & 124.5 \\
\hline & & Not congested & $22,291.6$ & 159.9 \\
\hline & \multicolumn{2}{|l|}{ Off-peak } & $70,725.3$ & 564.1 \\
\hline \multirow{3}{*}{ Large } & \multirow{2}{*}{ Peak } & Congested & 454.0 & 15.9 \\
\hline & & Not congested & $4,131.3$ & 12.6 \\
\hline & \multicolumn{2}{|l|}{ Off-peak } & $12,639.3$ & 59.3 \\
\hline \multirow{3}{*}{ Median } & \multirow{2}{*}{ Peak } & Congested & 22.0 & 0.2 \\
\hline & & Not congested & 56.3 & 0.1 \\
\hline & \multicolumn{2}{|l|}{ Off-peak } & 216.1 & 0.6 \\
\hline \multirow{3}{*}{ Small } & \multirow{2}{*}{ Peak } & Congested & 61.6 & 1.6 \\
\hline & & Not congested & 734.4 & 1.3 \\
\hline & \multicolumn{2}{|l|}{ Off-peak } & $2,195.7$ & 6.7 \\
\hline \multirow{3}{*}{ All urban } & \multirow{2}{*}{ Peak } & Congested & $3,860.4$ & 142.2 \\
\hline & & Not congested & $27,213.6$ & 173.9 \\
\hline & \multicolumn{2}{|l|}{ Off-peak } & $85,776.4$ & 630.7 \\
\hline \multicolumn{3}{|l|}{ Total } & $116,850.4$ & 946.7 \\
\hline
\end{tabular}

* Urban area size categories are based on population: very large - more than 3 million; large - 1 to 3 million; medium 0.5 to 1 million; small - less than 0.5 million.

${ }^{\dagger}$ Peak periods: 6:00 am to 9:30 am and 3:30 pm to 7:00 pm Monday through Friday; all others considered non-peak.

${ }^{\ddagger}$ A roadway section is considered congested during the peak periods if its Volume/Service Flow Ratio (V/SF) is greater than $95 \%$.

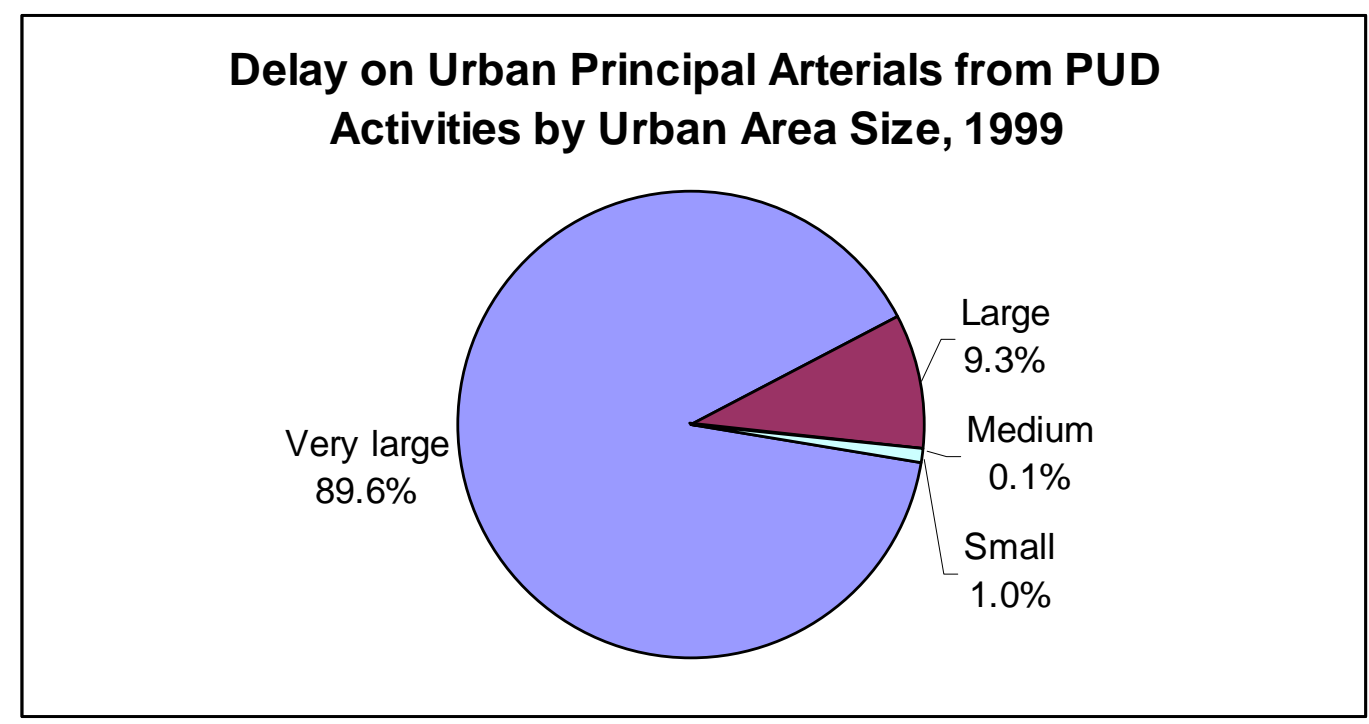

Fig. 28. Nearly 90 percent of the delay from PUD activities in 1999 occurred in very large urban areas. 


\section{Delay on Urban Principal Arterials from PUD Activities by Peak Period \& Traffic Condition, 1999}

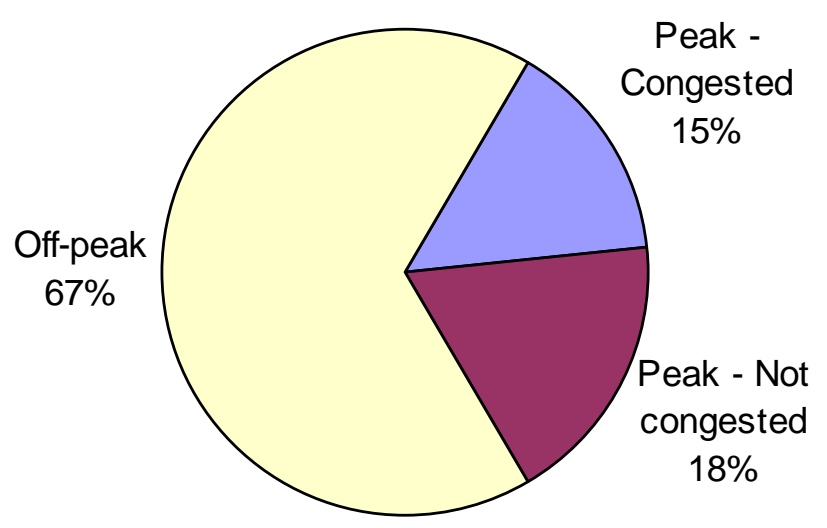

Fig. 29. Most of the delay on urban principal arterials from PUD activities in 1999 occurred in off-peak hours.

\subsection{RELIABILITY}

\subsubsection{Methodology}

Methodologies from the Highway Capacity Manual were used to estimate capacity loss and delay. These methodologies have evolved over the years and have been updated and enhanced continuously when new information or methodologies are made available. They are well established and accepted within the traffic engineering community. These methodologies are qualified as having a high degree of confidence. However, the final capacity reduction and delay estimates are significantly influenced by trip generation assumptions (described below) that are qualified as having a low level of confidence. Furthermore, using annual salary per square mile as a method for identifying dense commercial development with limited parking has a low level of confidence.

\subsubsection{Data \& Key Assumptions}

The Census ZIP Code Business Patterns dataset prepared annually by the U.S. Census Bureau is well established and is qualified as having high degree of confidence.

The Bureau of Labor Statistics (BLS) Covered Employment and Wages (CEW) Program data pertains to workers covered by State unemployment insurance (UI) laws and Federal civilian workers covered by the Unemployment Compensation for Federal Employees (UCFE) program. The data for both private sector and public sector workers are reported to the Bureau of Labor Statistics (BLS) by State employment security agencies as part of the Quarterly Census of Employment and Wages program. The CEW data are used as the benchmark source for employment by the Current Employment Statistics program and the Occupational Employment Statistics program. This information is qualified as having high degree of confidence.

Pickup and delivery trip generation rates are based on a single study cited in Truck Trip Generation Data: A Synthesis of Highway Practice, NCHRP Synthesis 298 and the ITE Trip Generation Handbook (a 
publication widely used by traffic engineers). However, the study is over 10 years old and only recorded observations for 30 sites ( 5 observations for each land use type). Thus, this information is qualified as having low degree of confidence.

Other urban truck pickup and delivery information used in TLC2, such as PUD activity percentages by day of week, percent of PUD events that block a traffic lane, and duration of lane-blocking activities, are also based on limited, outdated studies. Thus, this information is qualified as having low degree of confidence.

None of the information regarding PUD activities is differentiated by highway class. Therefore, TLC2 assumed that PUD events were equally likely to occur on all urban highway classes other than freeways. However, it is likely that a large share of lane-blocking PUD activities occur on lower-order highway classes (i.e., classes other than principal arterials). Therefore, this assumption may result in an overestimate of temporary capacity loss and delay on principal arterials. 


\section{RESULTS SUMMARY}

\subsection{RESULTS}

Temporary capacity losses due to work zones, crashes, breakdowns, adverse weather, sub-optimal signal timing, railroad crossings, toll facilities, and urban PUD activities resulted in over three and a half billion vehicle-hours of delay on U.S. freeways and principal arterials in 1999 (Fig. 30, Table 37). Assuming an average vehicle occupancy of 1.6 persons, this translates into about six billion person-hours of delay. Assuming an average value of time of $\$ 15$ per hour for each person impacted, temporary capacity losses produced about $\$ 55$ billion in lost time alone in 1999. Because conservative assumptions have been used throughout this analysis, and because several significant sources of delay have not been included, these estimates are believed to be a lower bound on the actual impacts of TLC.

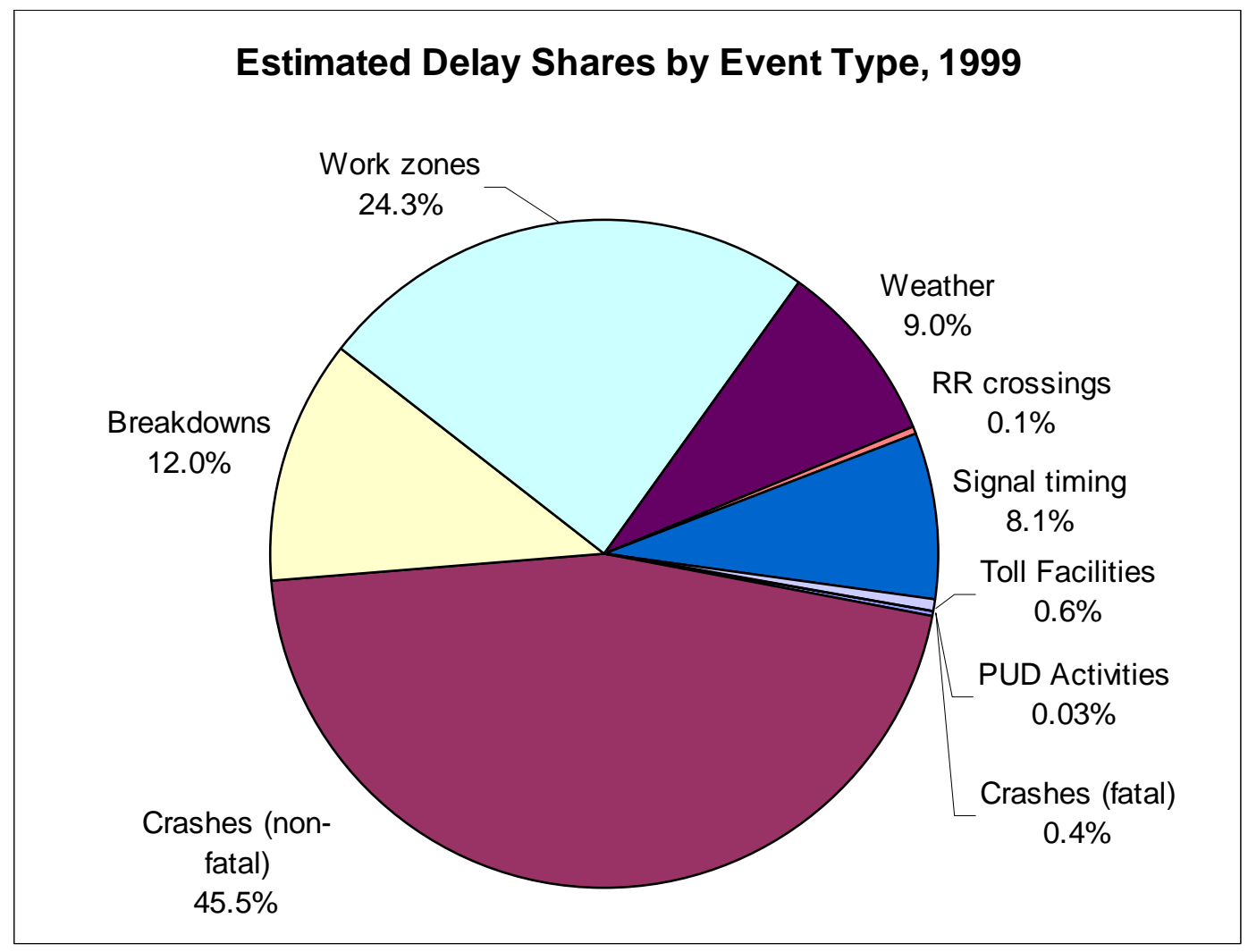

Fig. 30. Non-fatal crashes and work zones account for over two-thirds of the delay from temporary losses of capacity.

Non-fatal crashes were estimated to be the source of most delay from temporary capacity reductions, accounting for 45.5 percent of estimated delay. Work zones accounted for about a quarter of delay from TLC. Breakdowns, adverse weather, and signal timing were next, causing 12, 9, and 8.1 percent, respectively. Delay from toll facilities, fatal crashes, railroad crossings, and urban PUD activities combined were responsible for just over one percent of estimated delay. 
Table 37. Summary of capacity loss \& delay estimates for freeways \& principal arterials

\begin{tabular}{|c|c|c|c|c|}
\hline Event & $\begin{array}{l}\text { Total capacity loss* } \\
\text { (million vehicles) }\end{array}$ & $\begin{array}{l}\text { Total delay* } \\
\text { (million veh-hours) }\end{array}$ & $\begin{array}{l}\text { Average } \\
\text { delay/driver } \\
\text { (hours) }\end{array}$ & $\begin{array}{l}\text { Average } \\
\text { delay/event* } \\
\text { (veh-hours) }\end{array}$ \\
\hline Crashes & 3,290 & 1,680 & 9.0 & 506 \\
\hline Fatal & 30.5 & 13.7 & 0.1 & 754 \\
\hline Non-fatal & 3,250 & 1,660 & 8.9 & 505 \\
\hline Breakdowns & 7,480 & 440 & 2.4 & 15.9 \\
\hline Work zones & 8,350 & 889 & 4.7 & 836,000 \\
\hline Adverse weather & 20,900 & 330 & 1.8 & \\
\hline Fog & 410 & 5.79 & 0.03 & \\
\hline Rain & 929 & 44.8 & 0.2 & \\
\hline Snow & 3,290 & 43.8 & 0.2 & \\
\hline Ice & 16,200 & 236 & 1.3 & \\
\hline PUD activities & 117 & 0.95 & 0.01 & \\
\hline Railroad crossings & $\mathrm{NC}^{\ddagger}$ & 2.95 & 0.02 & \\
\hline Toll facilities & $\mathrm{NC}^{\ddagger}$ & 21.0 & 0.1 & \\
\hline Signal timing & 173,000 & 296 & 1.6 & 2,770 \\
\hline Total & & $3,660.0$ & 19.5 & \\
\hline Non-recurring delay & & $3,340.0$ & 17.9 & \\
\hline \multicolumn{5}{|c|}{$\begin{array}{l}\text { Due to significant uncertainty as to the accuracy of the estimates, all values in these columns are rounded to three } \\
\text { significant digits. Estimates in detailed tables in chapters 3-10 are not rounded; however, the number of decimal } \\
\text { places shown should not be considered an indication of the accuracy of those estimates. }\end{array}$} \\
\hline \multicolumn{5}{|c|}{$\begin{array}{l}\text { Delay/driver is averaged across all licensed drivers in the U.S. rather than for drivers actually delayed by each } \\
\text { crash. }\end{array}$} \\
\hline
\end{tabular}

The sources of delay are tabulated by highway type, urban area size, peak period versus off peak, and congestion level in Tables 38 through 40 . Surprisingly, over 85 percent of the delay estimated in TLC2 occurs in the off-peak period or on uncongested segments in the peak period. Whether or not in times and locations with recurring congestion, TLC 2 indicates that Americans lose 2.5 hours for every 1,000 miles of travel due to delay from incidents, work zones, bad weather, poor signal timing, railroad grade crossings, double-parked urban delivery vehicles, and toll booths. Delay is over 4 hours per 1,000 miles of travel in very large urban areas, about 3 hours and 45 minutes in large urban areas, over 2 hours in small and medium areas, and 45 minutes in rural areas. 
Table 38. Detailed delay summary.

\begin{tabular}{|c|c|c|c|c|c|c|c|c|c|c|c|c|c|c|}
\hline \multirow[b]{2}{*}{$\begin{array}{c}\text { Highway } \\
\text { type }\end{array}$} & \multirow[b]{2}{*}{$\begin{array}{c}\text { Urban } \\
\text { area } \\
\text { size* }^{*}\end{array}$} & \multirow[b]{2}{*}{$\begin{array}{c}\text { Peak } \\
\text { period }^{\dagger}\end{array}$} & \multirow[b]{2}{*}{$\begin{array}{c}\text { Congestion } \\
\text { level }^{*}\end{array}$} & \multirow[b]{2}{*}{ VMT } & \multicolumn{10}{|c|}{ Delay (million vehicle-hours) } \\
\hline & & & & & Total & $\begin{array}{c}\text { Fatal } \\
\text { crashes }^{\S}\end{array}$ & $\begin{array}{c}\text { Non- } \\
\text { fatal } \\
\text { crashes }\end{array}$ & $\begin{array}{l}\text { Break- } \\
\text { downs }\end{array}$ & $\begin{array}{l}\text { Work } \\
\text { zones }\end{array}$ & Weather & $\begin{array}{c}\text { Signal } \\
\text { timings }\end{array}$ & $\begin{array}{l}\text { Railroad } \\
\text { crossings }\end{array}$ & $\begin{array}{l}\text { Urban } \\
\text { PUD }\end{array}$ & $\begin{array}{c}\text { Toll } \\
\text { facilities }\end{array}$ \\
\hline \multirow{13}{*}{$\begin{array}{l}\text { Urban } \\
\text { freeways \& } \\
\text { expressways }\end{array}$} & \multirow{3}{*}{$\begin{array}{l}\text { Very } \\
\text { large }\end{array}$} & \multirow[t]{2}{*}{ Peak } & Congested & 22,345 & 130.9 & 0.0 & 96.6 & 1.1 & 19.4 & 13.8 & -- & -- & -- & -- \\
\hline & & & Not congested & 44,077 & 240.1 & 0.6 & 191.8 & 1.4 & 41.7 & 4.5 & -- & -- & -- & -- \\
\hline & & \multicolumn{2}{|c|}{ Off-peak } & 130,072 & 513.1 & 0.2 & 380.8 & 4.5 & 105.7 & 21.8 & -- & -- & -- & -- \\
\hline & \multirow[t]{3}{*}{ Large } & \multirow[t]{2}{*}{ Peak } & Congested & 14,854 & 104.6 & 0.003 & 49.3 & 0.2 & 45.7 & 9.4 & -- & -- & -- & -- \\
\hline & & & Not congested & 45,641 & 190.5 & 1.2 & 122.5 & 0.9 & 61.6 & 4.2 & -- & -- & -- & -- \\
\hline & & \multicolumn{2}{|c|}{ Off-peak } & 117,763 & 443.5 & 4.0 & 243.3 & 2.3 & $1,74.9$ & 19.0 & -- & -- & -- & -- \\
\hline & \multirow[t]{3}{*}{ Medium } & \multirow[t]{2}{*}{ Peak } & Congested & 3,950 & 21.5 & 0.0 & 9.9 & 0.05 & 9.4 & 2.1 & -- & -- & -- & -- \\
\hline & & & Not congested & 17,679 & 60.2 & 0.006 & 20.9 & 0.09 & 37.6 & 1.6 & -- & -- & -- & -- \\
\hline & & \multicolumn{2}{|c|}{ Off-peak } & 41,930 & 112.8 & 0.02 & 48.6 & 0.4 & 59.1 & 4.7 & -- & -- & -- & -- \\
\hline & \multirow[t]{3}{*}{ Small } & \multirow[t]{2}{*}{ Peak } & Congested & 3,883 & 7.1 & 0.0 & 0.7 & 0.04 & 5.5 & 0.8 & -- & -- & -- & -- \\
\hline & & & Not congested & 35,818 & 77.3 & 0.001 & 12.8 & 0.2 & 61.2 & 3.0 & -- & -- & -- & -- \\
\hline & & \multicolumn{2}{|c|}{ Off-peak } & 76,537 & 135.0 & 0.02 & 18.8 & 1.1 & 108.3 & 6.8 & -- & -- & -- & -- \\
\hline & \multicolumn{3}{|l|}{ Total } & 554,549 & $2,036.4$ & 6.1 & $1,196.1$ & 12.1 & 730.2 & 91.8 & -- & -- & -- & -- \\
\hline \multirow{13}{*}{$\begin{array}{l}\text { Urban other } \\
\text { principal } \\
\text { arterials }\end{array}$} & \multirow{3}{*}{$\begin{array}{l}\text { Very } \\
\text { large }\end{array}$} & \multirow[t]{2}{*}{ Peak } & Congested & 9,468 & 86.9 & $<0.001$ & 21.2 & 16.1 & 0.04 & 25.3 & 24.1 & 0.04 & 0.1 & -- \\
\hline & & & Not congested & 35,340 & 113.4 & 1.1 & 38.6 & 42.9 & 1.6 & 7.1 & 21.7 & 0.2 & 0.2 & -- \\
\hline & & Off-peak & & 87,730 & 288.2 & 0.5 & 79.9 & 89.3 & 0.8 & 49.7 & 67.1 & 0.5 & 0.6 & -- \\
\hline & Large & Peak & Congested & 4,963 & 36.4 & 0.0 & 10.9 & 4.1 & 0.1 & 11.7 & 9.7 & 0.02 & 0.02 & -- \\
\hline & & & Not congested & 28,882 & 85.9 & 0.1 & 36.1 & 31.0 & 0.1 & 5.8 & 12.8 & 0.1 & 0.01 & -- \\
\hline & & Off-peak & & 65,782 & 180.1 & 3.1 & 58.5 & 53.2 & 0.2 & 30.4 & 34.4 & 0.3 & 0.1 & -- \\
\hline & Medium & Peak & Congested & 2,168 & 14.1 & 0.0 & 2.8 & 1.1 & 0.005 & 4.3 & 5.9 & 0.008 & $<0.001$ & -- \\
\hline & & & Not congested & 12,049 & 26.1 & 0.1 & 8.3 & 7.3 & 0.1 & 5.0 & 5.3 & 0.05 & $<0.001$ & -- \\
\hline & & Off-peak & & 27,652 & 60.5 & 0.03 & 15.8 & 15.6 & 0.1 & 11.9 & 17.0 & 0.1 & $<0.001$ & -- \\
\hline & Small & Peak & Congested & 4,297 & 34.8 & $<0.001$ & 8.1 & 3.3 & 0.1 & 12.0 & 11.2 & 0.05 & 0.002 & -- \\
\hline & & & Not congested & 36,146 & 91.2 & 0.2 & 28.3 & 24.5 & 3.6 & 11.6 & 22.7 & 0.4 & 0.001 & -- \\
\hline & & Off-peak & & 78,244 & 201.8 & 1.9 & 59.2 & 43.8 & 3.2 & 37.0 & 55.7 & 1.0 & 0.007 & -- \\
\hline & Total & & & 392,721 & $1,219.5$ & 7.0 & 367.5 & 332.2 & 9.7 & 211.8 & 287.6 & 2.7 & 0.9 & -- \\
\hline Rural freeway & & Peak & Congested & 2,309 & 18.9 & 0.1 & 0.03 & 0.01 & 18.5 & 0.2 & -- & -- & -- & -- \\
\hline & & & Not congested & 86,020 & 40.9 & 0.05 & 5.6 & 0.1 & 31.5 & 3.7 & -- & -- & -- & -- \\
\hline & & Off-peak & & 171,875 & 105.7 & 0.03 & 10.6 & 0.3 & 86.5 & 8.3 & -- & -- & -- & -- \\
\hline & & Total & & 260,204 & 165.5 & 0.2 & 16.2 & 0.4 & 136.5 & 12.2 & -- & -- & -- & -- \\
\hline Rural other pr & acipal & Peak & Congested & 2,939 & 7.6 & 0.0 & 2.5 & 4.2 & 0.1 & 0.3 & 0.4 & 0.003 & -- & -- \\
\hline arterials & & & Not congested & 79,872 & 67.0 & 0.2 & 30.5 & 25.2 & 4.7 & 4.2 & 2.2 & 0.1 & -- & -- \\
\hline & & Off-peak & & 161,139 & 140.8 & 0.2 & 51.4 & 65.9 & 7.7 & 9.9 & 5.6 & 0.2 & -- & -- \\
\hline & & Total & & 243,950 & 215.4 & 0.4 & 84.3 & 95.3 & 12.5 & 14.3 & 8.2 & 0.3 & -- & -- \\
\hline Total & & & & $1,451,424$ & $3,657.9$ & 13.7 & $1,664.2$ & 440.0 & 889.0 & 330.1 & 295.8 & 2.9 & 0.9 & 21.0 \\
\hline
\end{tabular}


* Urban area size categories are based on population: very large - more than 3 million; large - 1 to 3 million; medium 0.5 to 1 million; small - less than 0.5 million.

${ }^{\dagger}$ Peak periods: 6:00 am to 9:30 am and 3:30 pm to 7:00 pm Monday through Friday; all others considered non-peak.

* A roadway section is considered congested during the peak periods if its Volume/Service Flow Ratio (V/SF) is greater than 95 percent in the peak hour.

$\S$ The GES data contain no fatal crashes for this urban area type, time period, and traffic condition. Therefore, capacity reduction and delay could not be

extrapolated for this cell within the table. While it is possible that a crash (or crashes) did occur under this condition, the probability of such a crash is very low.

Table 39. Summaries of delay by area type \& size, highway type, and traffic period \& congestion level.

\begin{tabular}{|c|c|c|c|c|c|c|c|c|c|c|c|}
\hline & \multirow{2}{*}{$\begin{array}{c}\text { Share } \\
\text { of } \\
\text { total }\end{array}$} & \multicolumn{10}{|c|}{ Delay in million vehicle hours } \\
\hline & & Total & $\begin{array}{c}\text { Fatal } \\
\text { crashes }\end{array}$ & $\begin{array}{c}\text { Non-fatal } \\
\text { crashes }\end{array}$ & $\begin{array}{l}\text { Break- } \\
\text { downs }\end{array}$ & $\begin{array}{l}\text { Work } \\
\text { zones } \\
\end{array}$ & Weather & $\begin{array}{c}\text { Signal } \\
\text { timings } \\
\end{array}$ & $\begin{array}{l}\text { Railroad } \\
\text { crossings }\end{array}$ & $\begin{array}{c}\text { Urban } \\
\text { PUD } \\
\end{array}$ & $\begin{array}{c}\text { Toll } \\
\text { facilities } \\
\end{array}$ \\
\hline Total & $100 \%$ & $3,657.9$ & 13.7 & $1,664.2$ & 440.0 & 889.0 & 330.1 & 295.8 & 2.9 & 0.95 & 21.0 \\
\hline \multicolumn{12}{|l|}{ By area type $\&$ size $^{*}$} \\
\hline Urban - Very large & $38 \%$ & $1,372.6$ & 2.5 & 808.8 & 155.3 & 169.3 & 122.2 & 112.9 & 0.7 & 0.9 & -- \\
\hline Urban - Large & $28 \%$ & $1,041.0$ & 8.4 & 520.6 & 91.6 & 282.6 & 80.5 & 56.8 & 0.4 & 0.09 & -- \\
\hline Urban - Medium & $8 \%$ & 295.2 & 0.1 & 106.2 & 24.5 & 106.3 & 29.6 & 28.2 & 0.2 & 0.001 & -- \\
\hline Urban - Small & $15 \%$ & 547.1 & 2.1 & 128.0 & 72.9 & 181.8 & 71.3 & 89.7 & 1.4 & 0.01 & -- \\
\hline Rural & $10 \%$ & 380.9 & 0.6 & 100.5 & 95.7 & 149.0 & 26.5 & 8.2 & 0.3 & -- & -- \\
\hline \multicolumn{12}{|l|}{ By highway type } \\
\hline Urban freeways \& expressways & $56 \%$ & $2,036.4$ & 6.1 & $1,196.1$ & 12.1 & 730.2 & 91.8 & -- & -- & -- & -- \\
\hline Urban other principal arterials & $33 \%$ & $1,219.5$ & 7.0 & 367.5 & 332.2 & 9.7 & 211.8 & 287.6 & 2.7 & 1.0 & -- \\
\hline Rural Freeways & $5 \%$ & 165.5 & 0.2 & 16.2 & 0.4 & 136.5 & 12.2 & -- & -- & -- & -- \\
\hline Rural other principal arterials & $6 \%$ & 215.4 & 0.4 & 84.3 & 95.3 & 12.5 & 14.3 & 8.2 & 0.3 & -- & -- \\
\hline \multicolumn{12}{|l|}{ By period \& congestion level $^{\dagger}$} \\
\hline Peak - Congested & $13 \%$ & 462.8 & 0.1 & 201.9 & 30.2 & 98.9 & 80.1 & 51.3 & 0.1 & 0.1 & -- \\
\hline Peak - Not congested & $27 \%$ & 992.5 & 3.6 & 495.4 & 133.5 & 243.6 & 50.6 & 64.8 & 0.8 & 0.2 & -- \\
\hline Off-peak & $60 \%$ & $2,181.5$ & 10.0 & 966.9 & 276.3 & 546.5 & 199.5 & 179.7 & 2.0 & 0.6 & -- \\
\hline
\end{tabular}

* Urban area size categories are based on population: very large - more than 3 million; large - 1 to 3 million; medium 0.5 to 1 million; small - less than 0.5 million.

${ }^{\dagger}$ Peak periods: 6:00 am to 9:30 am and 3:30 pm to 7:00 pm Monday through Friday; all others considered non-peak. A roadway section is considered congested during the peak periods if its Volume/Service Flow Ratio (V/SF) is greater than 95 percent in the peak hour. 
Table 40. Summaries of delay per thousand miles of travel.

\begin{tabular}{|c|c|c|c|c|c|c|c|c|c|c|c|}
\hline & \multirow[b]{2}{*}{$\begin{array}{c}\text { VMT } \\
\text { (millions) }\end{array}$} & \multicolumn{10}{|c|}{ Delay in hours per thousand miles of travel } \\
\hline & & Total & $\begin{array}{c}\text { Fatal } \\
\text { crashes }\end{array}$ & $\begin{array}{l}\text { Non-fatal } \\
\text { crashes }\end{array}$ & $\begin{array}{l}\text { Break- } \\
\text { downs }\end{array}$ & $\begin{array}{l}\text { Work } \\
\text { zones }\end{array}$ & Weather & $\begin{array}{l}\text { Signal } \\
\text { timings }\end{array}$ & $\begin{array}{l}\text { Railroad } \\
\text { crossings }\end{array}$ & $\begin{array}{l}\text { Urban } \\
\text { PUD }\end{array}$ & $\begin{array}{c}\text { Toll } \\
\text { facilities }\end{array}$ \\
\hline Total & $1,451,424$ & 2.520 & 0.009 & 1.147 & 0.303 & 0.613 & 0.227 & 0.204 & 0.002 & 0.001 & 0.014 \\
\hline \multicolumn{12}{|l|}{ By area type $\&$ size* } \\
\hline Urban - Very large & 329,032 & 4.172 & 0.008 & 2.458 & 0.472 & 0.515 & 0.371 & 0.343 & 0.002 & 0.003 & - \\
\hline Urban - Large & 277,885 & 3.746 & 0.030 & 1.873 & 0.330 & 1.017 & 0.290 & 0.205 & 0.001 & 0.000 & - \\
\hline Urban - Medium & 105,428 & 2.800 & 0.001 & 1.007 & 0.232 & 1.009 & 0.281 & 0.267 & 0.002 & 0.000 & - \\
\hline Urban - Small & 234,925 & 2.329 & 0.009 & 0.545 & 0.310 & 0.774 & 0.303 & 0.382 & 0.006 & 0.000 & - \\
\hline Rural & 504,154 & 0.756 & 0.001 & 0.199 & 0.190 & 0.296 & 0.053 & 0.016 & 0.001 & - & - \\
\hline \multicolumn{12}{|l|}{ By highway type } \\
\hline $\begin{array}{l}\text { Urban freeways \& } \\
\text { expressways }\end{array}$ & 554,549 & 3.672 & 0.011 & 2.157 & 0.022 & 1.317 & 0.166 & - & - & - & - \\
\hline $\begin{array}{l}\text { Urban other principal } \\
\text { arterials }\end{array}$ & 392,721 & 3.105 & 0.018 & 0.936 & 0.846 & 0.025 & 0.539 & 0.732 & 0.007 & 0.002 & - \\
\hline Rural freeways & 260,204 & 0.636 & 0.001 & 0.062 & 0.002 & 0.525 & 0.047 & - & - & - & - \\
\hline $\begin{array}{l}\text { Rural other principal } \\
\text { arterials }\end{array}$ & 243,950 & 0.883 & 0.002 & 0.346 & 0.391 & 0.051 & 0.059 & 0.034 & 0.001 & - & - \\
\hline \multicolumn{12}{|c|}{ By period \& congestion level $^{\dagger}$} \\
\hline $\begin{array}{l}\text { Peak period - } \\
\text { Congested }\end{array}$ & 71,176 & 6.502 & 0.002 & 2.837 & 0.424 & 1.390 & 1.125 & 0.721 & 0.002 & 0.002 & - \\
\hline $\begin{array}{l}\text { Peak period - Not } \\
\text { congested }\end{array}$ & 421,524 & 2.355 & 0.009 & 1.175 & 0.317 & 0.578 & 0.120 & 0.154 & 0.002 & 0.000 & - \\
\hline Off-peak & 958,724 & 2.275 & 0.010 & 1.008 & 0.288 & 0.570 & 0.208 & 0.187 & 0.002 & 0.001 & - \\
\hline
\end{tabular}

* Urban area size categories are based on population: very large - more than 3 million; large - 1 to 3 million; medium 0.5 to 1 million; small - less than 0.5 million.

${ }^{\dagger}$ Peak periods: 6:00 am to 9:30 am and 3:30 pm to 7:00 pm Monday through Friday; all others considered non-peak. A roadway section is considered congested during the peak periods if its Volume/Service Flow Ratio (V/SF) is greater than 95 percent in the peak hour. 
In terms of total delay from TLC events, urban areas were estimated to have experienced more delay than rural areas, and larger urban areas experienced more delay than smaller ones. Although Figure 31 shows that medium-sized urban areas experienced much less total delay than might have been expected (based on area type and size), when the amount of travel within each area type/size is considered, estimated delay for this urban area size follows the trend for other urban areas (Fig. 32).

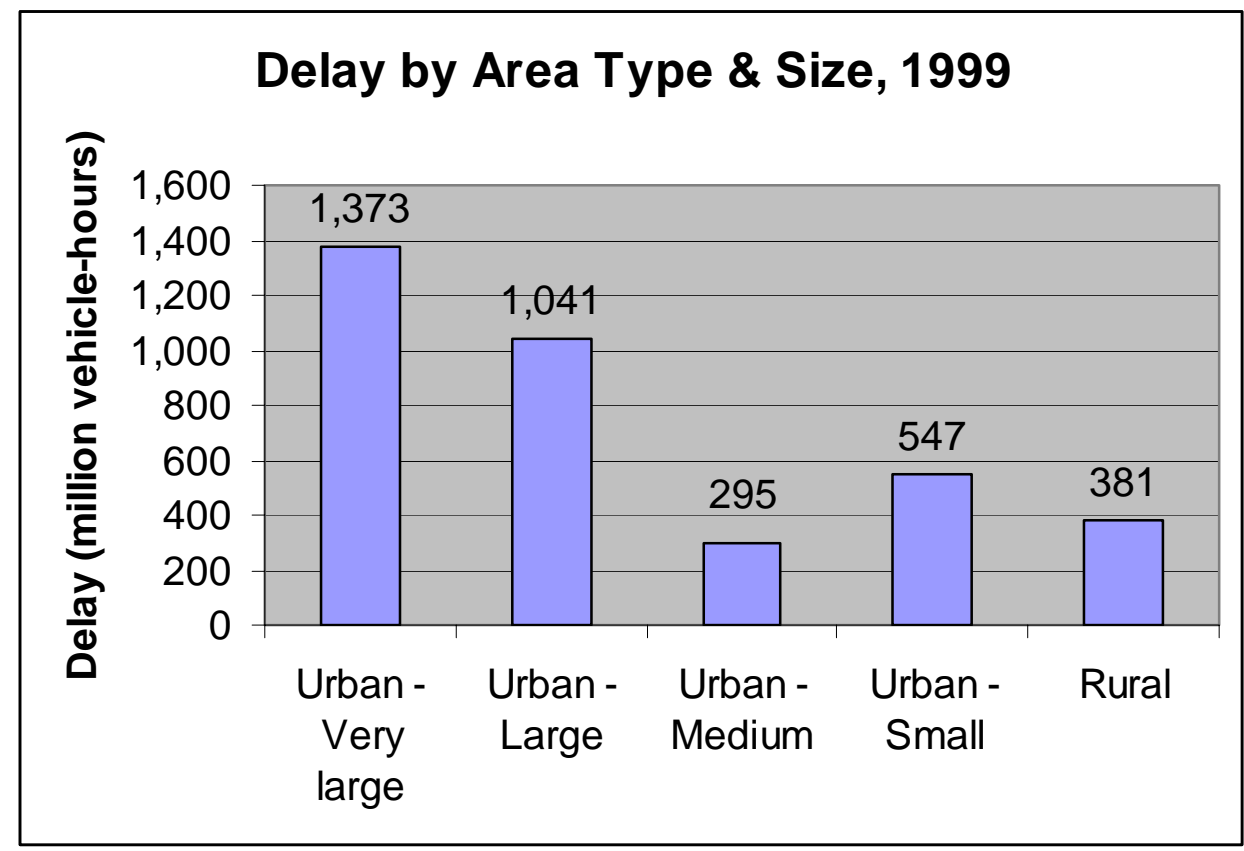

Fig. 31. Medium-sized urban areas experienced the least amount of delay from TLC events in 1999.

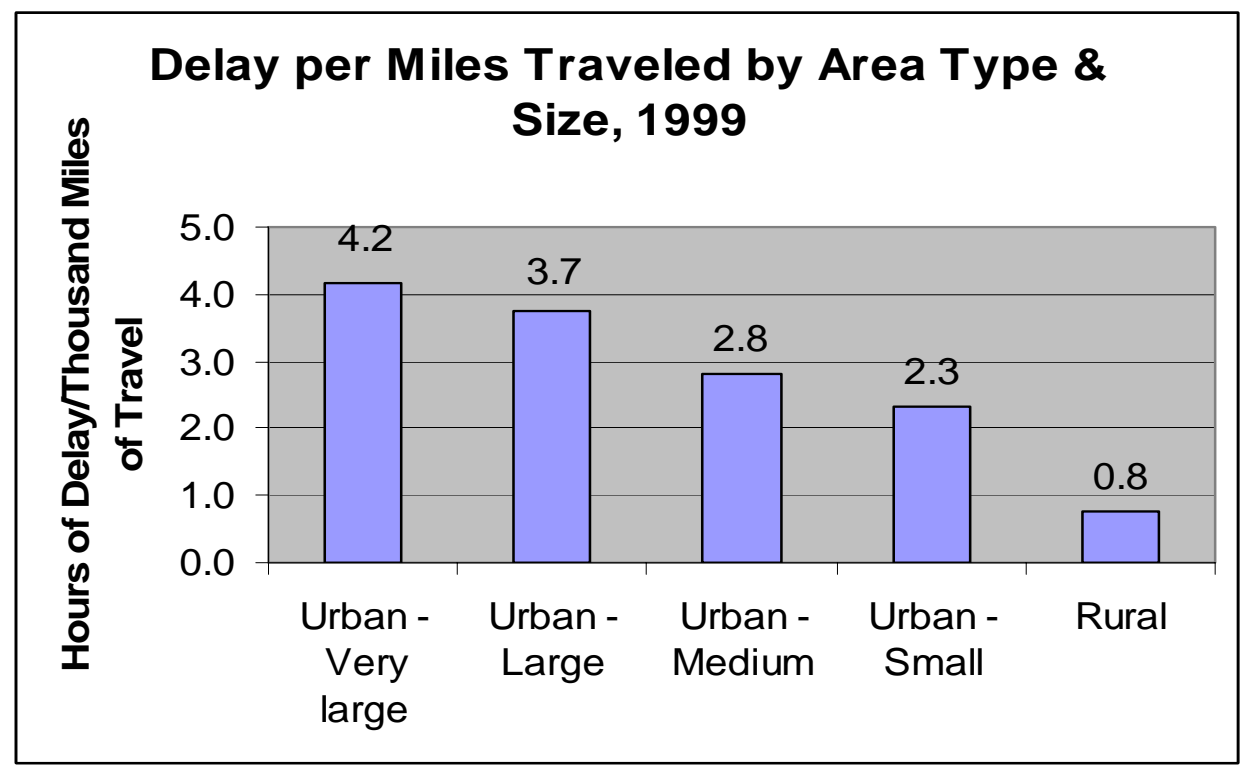

Fig 32. When the amount of travel in each area type/size is considered, the delay experienced in medium-sized urban areas is more in line with size (in terms of population). 
In urban areas, more delay from TLC events was realized on freeways than on principal arterials, but in rural areas the opposite was true: other principal arterials experienced slightly more delay (Fig. 33). This also held true when the amount of vehicle travel on these highway types was considered.

\section{Delay by Highway Type, 1999}

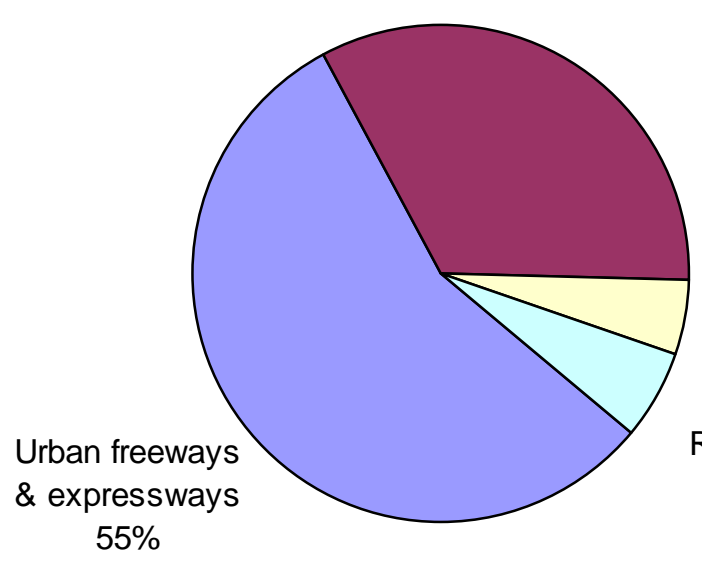

$$
\begin{aligned}
& \text { Urban other } \\
& \text { principal } \\
& \text { arterials } \\
& 34 \%
\end{aligned}
$$

Rural freeways $5 \%$

Rural other principal arterials

$6 \%$

Fig. 33. Delay from TLC events was most prevalent on urban freeways.

Most of the delay from temporary capacity losses (60 percent) was experienced during off-peak periods (Fig. 34). About 27 percent of delay was experienced on uncongested highways during peak periods, and nearly 13 percent was experienced on congested highways during peak-periods.

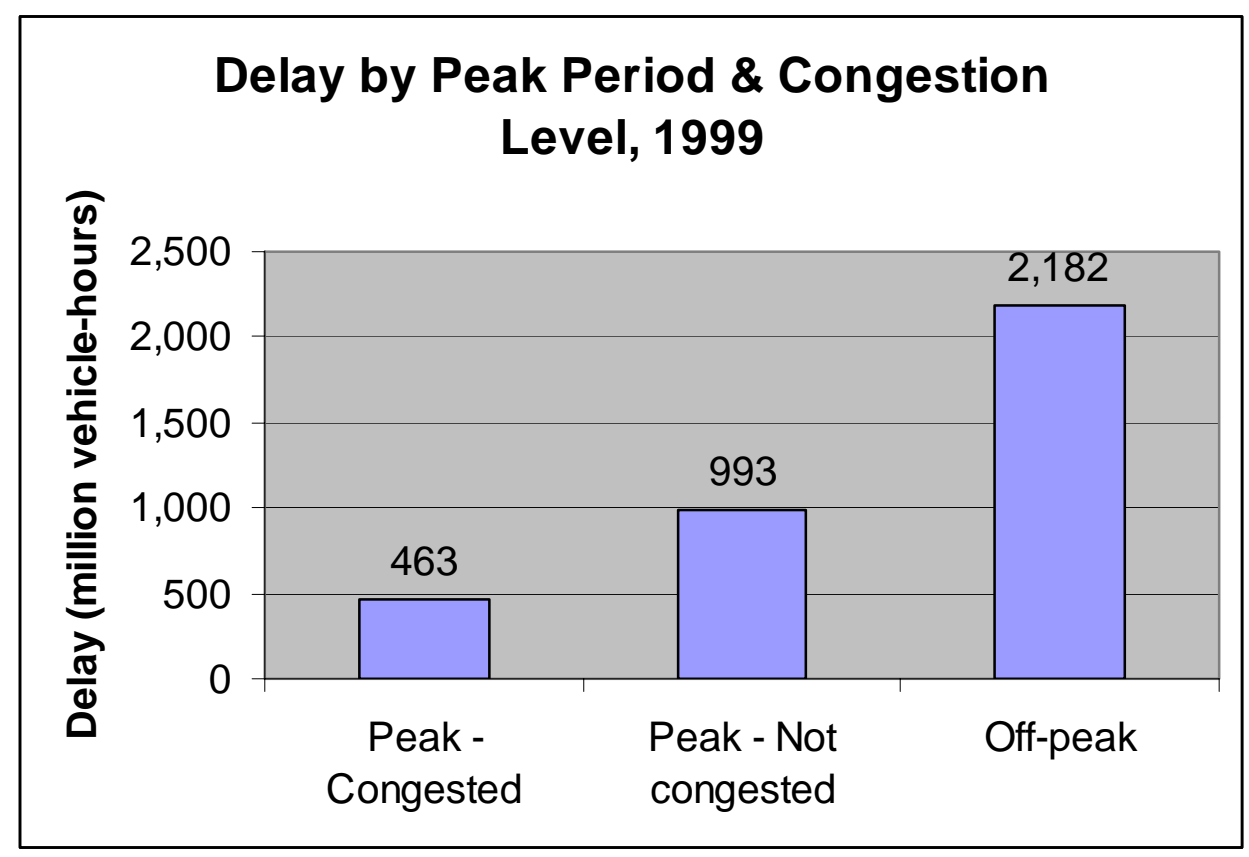

Fig. 34. Most delay from TLC events occurred during off-peak periods. 
While total delay from temporary events is least on congested highway segments (Fig. 34), the picture is reversed when calculated per mile of travel (Fig. 35). This explains why unexpected delay seems worse to people traveling in the peak period. While the ratio of congested VMT to total VMT seems low in Tables 38 and 40 and figures 34 and 35, the TLC2 study overestimates the amount of VMT on congested highway segments because VMT on a segment congested in the peak hour is classified as congested for the entire peak period.

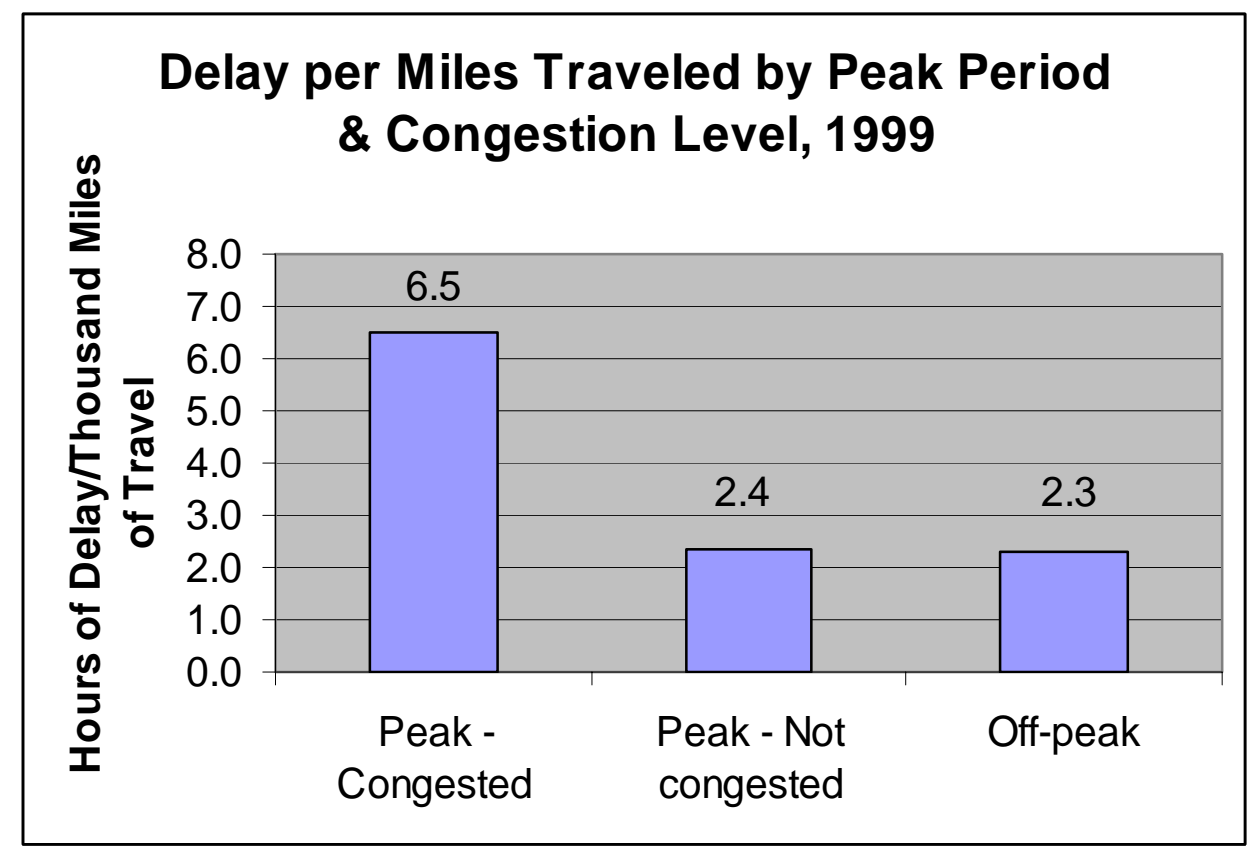

Fig. 35. On a per-mile-of-travel basis, delay from TLC events was more likely to occur during peak periods on congested roadways.

\subsection{COMPARING TLC2 RESULTS TO OTHERS}

This study has broken new ground in developing the first "bottom-up," nationwide estimates of temporary losses of highway capacity and resulting delay. In the course of the research, much has been learned about both data sources and methodologies that can be applied to improve and expand information about TLC impacts.

The TLC2 estimates were compared to two sets of estimates by Texas Transportation Institute (TTI), including estimates of incident delay for 85 urban areas in 1999 in the 2004 Urban Mobility Study (Schrank and Lomax 2004) and unpublished estimates of incident delay in 1999 for all FHWArecognized urban areas in support of FHWA's annual Performance and Accountability Report. FHWArecognized urban areas are urbanized areas defined by the U.S. Bureau of the Census with populations of at least 50,000 - local transportation officials may adjust Census-defined borders. There were just over 400 urban areas in 1999. The TTI estimates are based on the most recent TTI procedures applied to 1999 data, with more extensive quality checks for the published estimates for the 85 areas than for the unpublished estimates for all urban areas. Both published and unpublished TTI estimates of delay per person are converted to vehicle delay with TTI's vehicle occupancy factor of 1.25. The comparable TLC2 estimates are for vehicle delay from crashes and breakdowns, but cover all urban and rural areas. Neither TLC2 nor TTI estimates cover roads other than freeways and other major arteries. 
The delay estimates for crashes and breakdowns in TLC2 are slightly higher than both sets of incident delay estimates from TTI (Fig. 36). The differences between TLC2 and TTI for all urban areas vary slightly by urban area size (Fig. 37). A complete assessment of the causes of differences between TTI and TLC2 estimates is beyond the scope of this study.

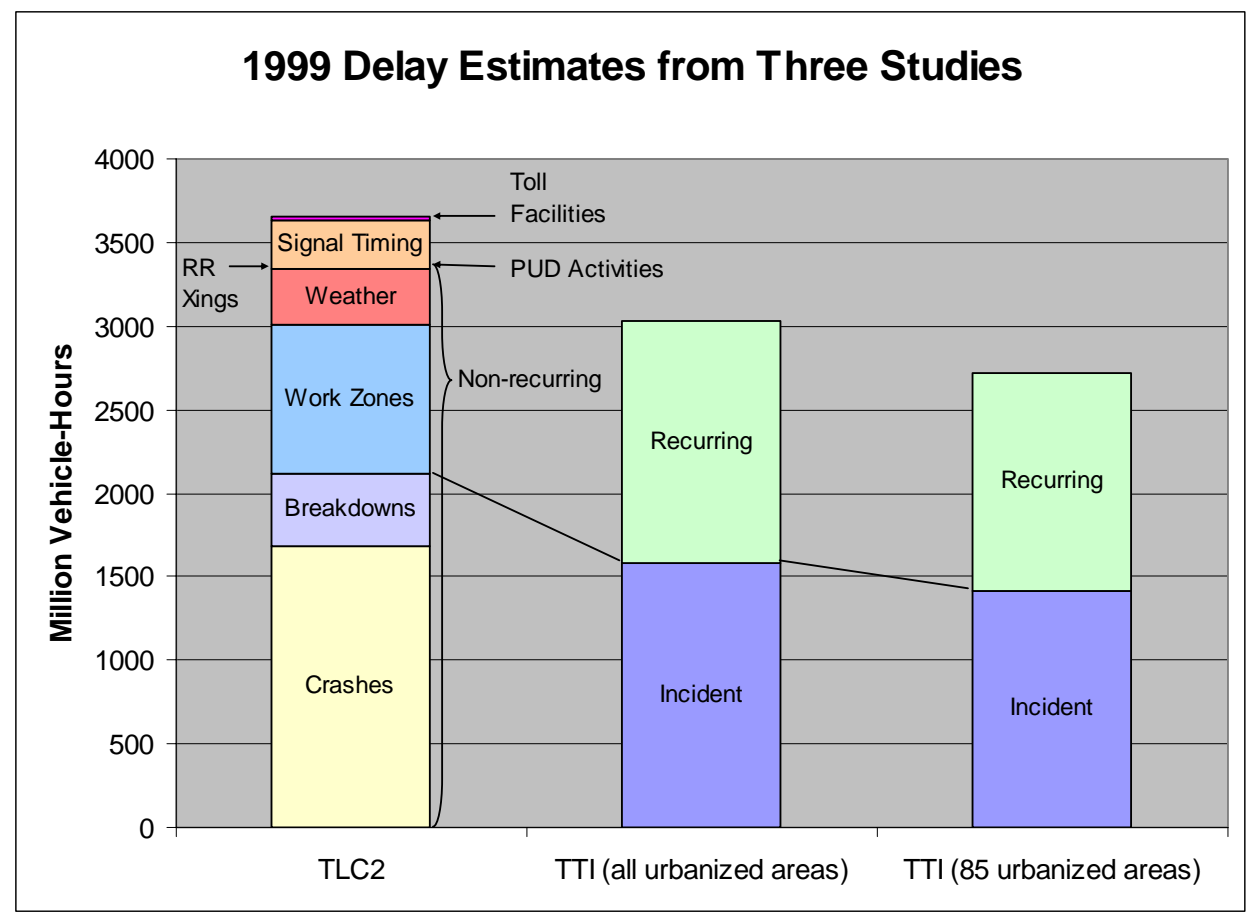

Fig. 36. A comparison of three studies that estimate delay: TLC2, TTI's Urban Mobility Study (85 urbanized areas), and TTI's estimate for the FHWA Office of Operations (all urbanized areas).

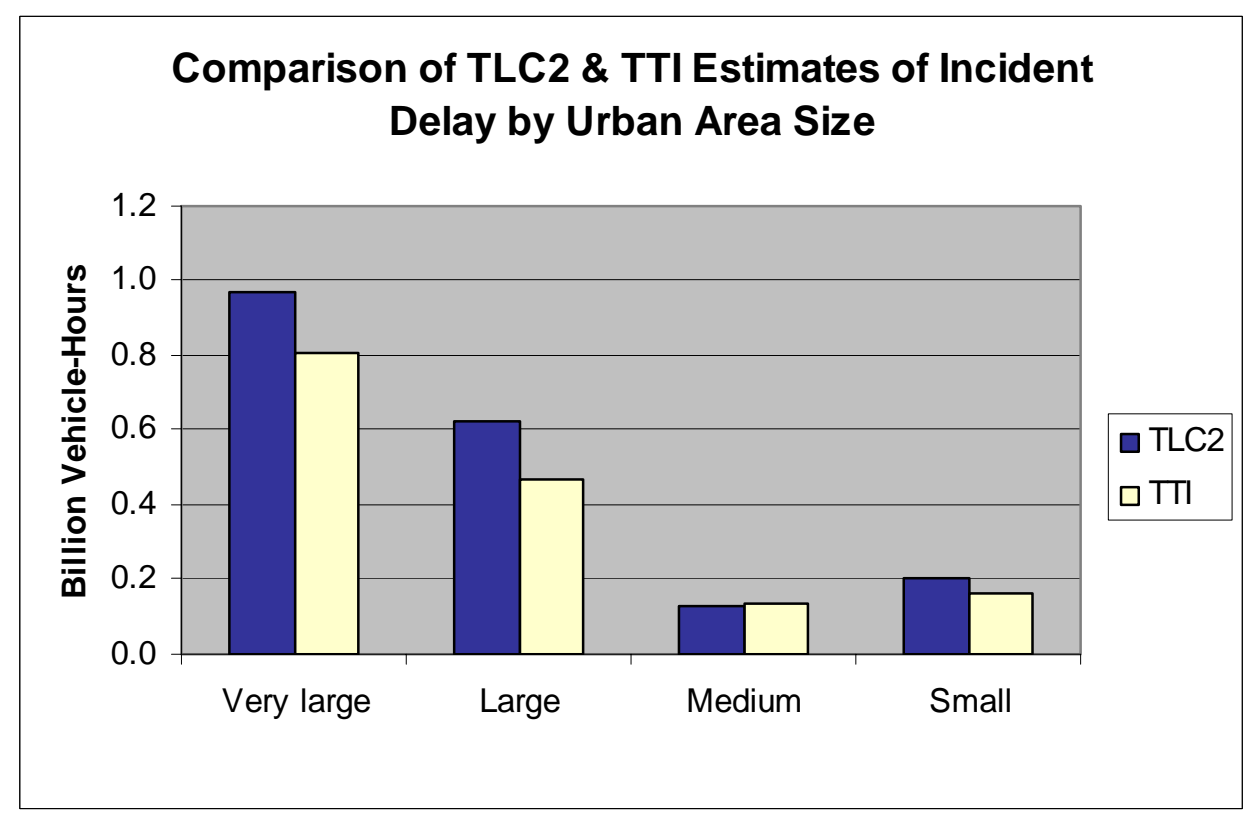

Fig. 37 TLC2 delay estimates are somewhat higher than TTI's (all-urban-area study) for most urban area sizes. 


\subsection{A COMPOSITE PICTURE OF DELAY}

No single empirical or modeling study provides a comprehensive estimate of all sources of delay. However, a comprehensive picture can be assembled by combining elements of TLC2 and TTI's unpublished estimates for FHWA's annual Performance and Accountability Report. The resulting picture is a very approximate composite because very different methods are used in TLC2 and the TTI studies. TTI estimates recurring delay from weekday commuting peaks, while TLC2 estimates recurring delay from two elements (suboptimal signal timing and tollbooths) not covered by TTI. TTI estimates nonrecurring delay from relationships between incident delay and recurring delay in urban areas, while TLC2 uses a bottom-up approach to estimating nonrecurring delay from a variety of sources in both urban and rural areas.

The composite picture uses TTI's estimate of recurring delay for all urban areas, the TLC2 estimate of recurring delay for suboptimal signal timing and tollbooths, and the more comprehensive TLC2 estimates of nonrecurring delay. The resulting 5.1 billion hours of delay is 35 percent recurring and 65 percent nonrecurring. Recurring delay is a higher percentage in the larger cities (Fig. 38).

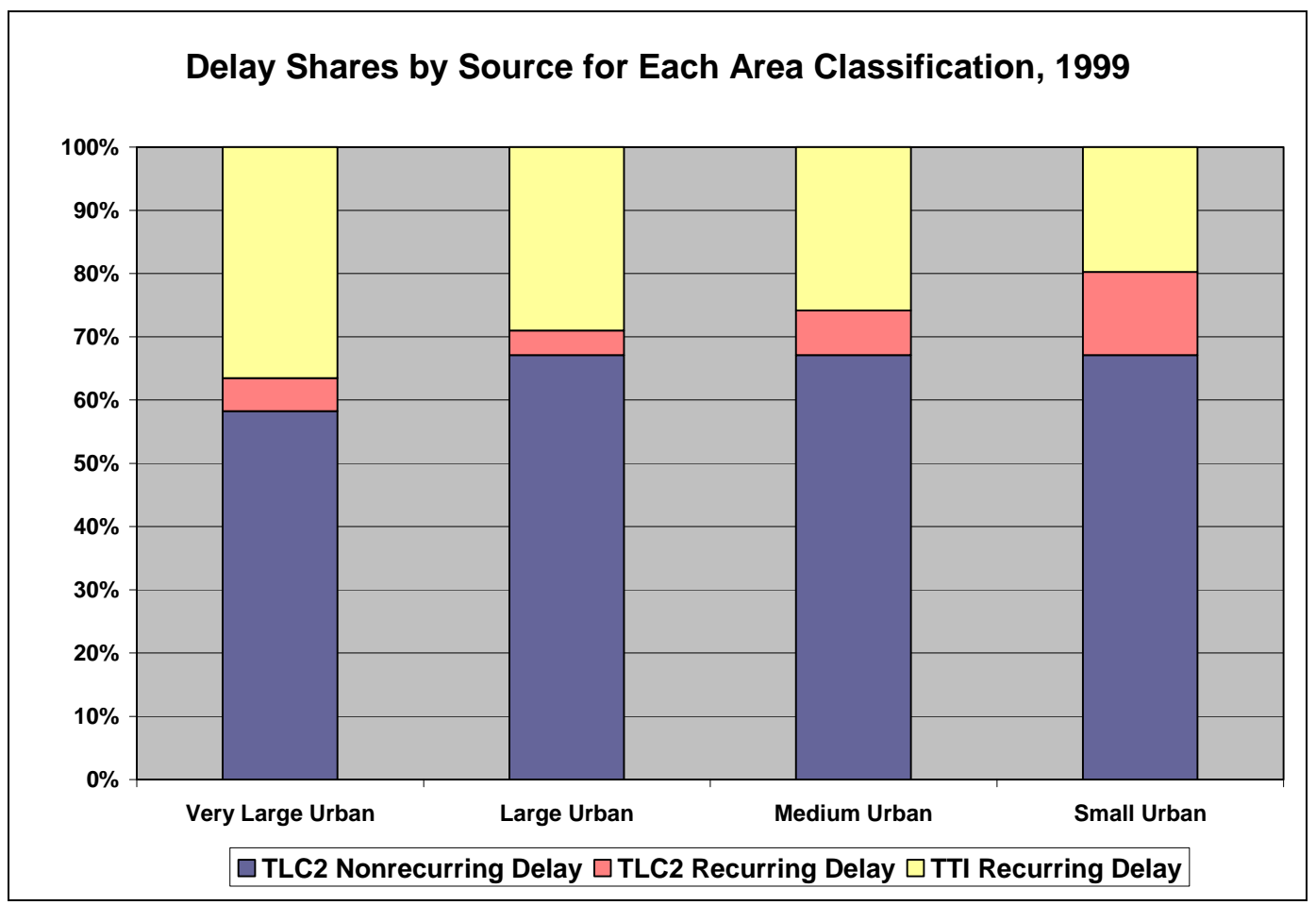

Fig. 38. Very large urban areas had a somewhat greater share of recurring delay than other area types.

The combined TLC2-TTI estimate suggests a slightly higher contribution of nonrecurring delay than the composite picture compiled for the Federal Highway Administration from a variety of studies and professional judgments. The relative delay shares by source in these composite pictures are within 10 percentage points in every category except incidents (Table 41). 
Table 41. Composite estimates of sources of delay

\begin{tabular}{|c|c|c|}
\hline \multirow[b]{2}{*}{ Source category } & \multicolumn{2}{|c|}{ Delay share by source } \\
\hline & $\begin{array}{c}\text { TTI \& TLC2 } \\
\text { composite }\end{array}$ & $\begin{array}{c}\text { Traffic Congestion } \\
\text { and Reliability report* }\end{array}$ \\
\hline TTI recurring delay & $33 \%$ & \\
\hline Bottlenecks & & $40 \%$ \\
\hline Incidents & $39 \%$ & $25 \%$ \\
\hline Work zones & $16 \%$ & $10 \%$ \\
\hline Bad weather & $6 \%$ & $15 \%$ \\
\hline Suboptimal signal timing & $5 \%$ & $5 \%$ \\
\hline Other & $1 \%$ & $5 \%$ \\
\hline Total & $100 \%$ & $100 \%$ \\
\hline
\end{tabular}

Neither composite picture is complete and adequately supported with empirical observations. Recurring congestion from weekend and holiday travel in all areas and recurring weekday congestion in rural areas are poorly captured, if at all. The full effects of bottlenecks, the extent and intensity of most forms of temporary capacity reductions, and the consequences of dramatic increases in trucking are not adequately understood or based on robust empirical studies. Delay on roads smaller than freeways and other major arteries is another unexplored part of the picture. Substantial data collection and analysis are necessary before a complete picture of delay can be framed. 


\section{NEXT STEPS}

The TLC and TLC2 studies represent an initial attempt to estimate the loss of capacity and delay due to short-term events. Due to the scope of these studies, time and effort constraints, and limitations in available data, there are areas in which TLC delay estimates could be significantly improved or expanded. This chapter discusses further activities that would improve the initial estimates.

\subsection{ESTIMATE IMPACTS FROM SIMULTANEOUS CAPACITY-REDUCING EVENTS}

TLC2 could be extended to estimate capacity reduction and delay resulting from simultaneous capacityreducing events, such as a breakdown or crash in a work zone or during a snowstorm. These types of events might cause capacity losses and delays that are much longer than those that result when no interaction is considered.

\subsection{DELPHI SURVEY}

A number of assumptions were made in the TLC study, some of which affected the methodologies and some of which affected equation parameters. Because of uncertainties about many of the key parameters of this analysis, it would be useful to conduct a Delphi survey of traffic engineers and other experts regarding the values they believe are correct. In addition to improving assumptions for key variables in the current methodology, the results of the Delphi survey could be used to determine distributions of values for sensitivity analysis.

\subsection{METHODOLOGY FOR ANALYZING DETAILED DELAY ESTIMATES}

The capacity loss and delay are estimated for detailed breakdowns by area type and size, highway type, and peak period/congestion level. It is often difficult to understand the reasons for the results $\mathrm{A}$ methodology could be developed to help answer why the models in TLC2 produced a given result for a specific category. This would improve our understanding of both the TLC2 models and the factors that affect specific delay estimates.

\subsection{SENSITIVITY ANALYSES}

The national-level scope of the TLC study and its novelty made it necessary to make a large number of assumptions regarding key parameters. Confidence in the resulting estimates could be greatly increased by performing a sensitivity analysis to determine which variables most affect capacity loss and delay estimates.

\subsection{METHODS FOR ESTIMATING THE 4 RS}

As stated often in this report, the TLC study did not attempt to assess impacts of re-routing, rescheduling, reduced mobility, and reduced reliability on delay. Analytical methods for estimating rerouting, rescheduling, reduced mobility, and reduced reliability could be developed to give a more complete and accurate analysis of TLC impacts. 


\subsection{ASSESS POLICY AND TECHNOLOGY IMPACTS}

At present, the TLC study attempts to estimate only the quantity or magnitude of delay from temporary losses of capacity. Of greater significance for policy decision-making would be estimates of the potential benefits of various policy and technology alternatives, such as work zone management strategies, electronic toll technologies, and other alternatives, that may reduce capacity losses and their impacts.

\subsection{METHODOLOGY IMPROVEMENTS}

During the TLC study, several areas in which the initial methodologies could be improved were identified:

- Using probability distributions to assign incident duration instead of using mean duration

- Obtaining more information about crashes on arterials and adjust the methodology to compute delays accordingly

- For crashes at intersections, estimating the impacts on transversal roadway segments

- Including the effects of crossover work zones, where the capacity of lanes in the other direction are affected by the construction

- Improving the delay estimation methodology by considering demand reductions downstream of a capacity-reducing event

- Using stochastic network simulation models to validate the deterministic delay calculation

\subsection{DATA IMPROVEMENTS}

The completeness and reliability of TLC estimates could be improved by using, compiling, and/or collecting additional data.

- Refine data for demand distribution multipliers for freeways and generate demand distribution multipliers for arterials instead of using freeway distributions as a surrogate.

- Several other data sources for crashes and breakdowns on freeways were used as a surrogate for arterials. These data need to be validated for arterials or new data sources need be identified.

- Collect more data on delay and capacity loss estimation due to vehicle breakdowns. This could also lead to methodology improvements.

- Further study of the Monte Carlo simulations used with GES data to estimate delay and capacity loss due to non-fatal crashes should be performed.

\subsection{CONSIDER PUBLISHING TLC STUDY AS AN ANNUAL REPORT}

The TLC study methodology could be used to produce an annual report on delays due to temporary capacity losses. A historical time series of nation-wide delay due to TLC could also be estimated. 


\section{REFERENCES}

Aherns, G.A., K.W. Forstall, R.U. Guthrie, and B.J. Ryan. 1977. "Analysis of Truck Deliveries in a Small Business District," Transportation Research Record 637, Transportation Research Board, National Research Council, Washington, DC, pp. 81-86.

American Trucking Association and Cambridge Systematics, Inc. 1991. Incident Management: Challenges, Strategies, and Solutions for Advancing Safety and Roadway Efficiency.

Aron, M., M. Ellenerg, and P. Veyre. 1994. "Weather Related Traffic Management," Towards an Intelligent Transport System, Vol. 3, Proceedings of the First World Congress on Applications of Transport Telematics and Intelligent Vehicle-Highway Systems, Paris, November 30-December 3, pp. 1089-1096.

Blumentritt, C.W., D.W. Ross, J. Glazer, C. Pinnell, and W.R. McCasland. 1981. Guidelines for Selection of Ramp Control Systems (NCHRP Report 232), Transportation Research Board, National Research Council, Washington DC, May.

Brilon, W., and M. Ponzlet. 1995. Auswirkungen on Zeitlich Veraenderlichen Leistungsfaehigkeiten, Schlussbericht. Lehrstuhl fur Verkeshrswesen, Ruhr Universitat Bochum.

Cambridge Systematics, Inc. 2001. ITS Deployment Analysis System (IDAS) - User's Manual, Oakland, California, November (http://idas.camsys.com/documentation.htm).

Cambridge Systematics, Inc. 2004. Traffic Congestion and Reliability: Linking Solutions to Problems. Prepared for the Federal Highway Administration, July, pp. 2-4 (http://www.ops.fhwa.dot.gov/congestion_report/index.htm).

Chin, S.M., O. Franzese, D.L. Greene, H.L. Hwang, and R.C. Gibson. 2002. Temporary Losses of Highway Capacity and Impacts on Performance, ORNL/TM-2002/3, Oak Ridge National Laboratory, Oak Ridge, Tennessee.

Chin, S.M., A. A. Gibson, D. L. Greene, and H. L. Hwang. 2000. "Real-time Indicators of Vehicle Kilometers of Travel And Congestion: One Year of Experience," Transportation Research Record No. 1719: Transportation Data, Statistics, and Information Technology, Transportation Research Board, National Research Council, Washington DC.

Chin, S. M., D. L. Greene, J. Hopson, H. L. Hwang, and B. Thompson. 1999. "Toward National Indicators of Vehicle Travel And Traffic Congestion Based on Real-time Traffic Data," Transportation Research Record No. 1660, Part 2: Improving Transportation Data, Transportation Research Board, National Research Council, Washington DC.

Dixon, K.K., J.E. Hummer, and A.R. Lorscheider. 1996. "Capacity for North Carolina Freeway Work Zones," Transportation Research Record 1529, Transportation Research Board, National Research Council, Washington DC, pp. 27-34.

Gannett Fleming, Inc. 1993. Technical Memorandum No. 2: Truck/Taxi Travel Survey, Tampa, Florida, July. 
Giuliano, G. 1989. "Incident Characteristics, Frequency, and Duration on a High Volume Urban Freeway," Transportation Research Part A: General 23(5), Transportation Research Board, National Research Council, Washington DC, pp 387-96.

Fischer, M.J. and M. Han. 2001. Truck Trip Generation Data: A Synthesis of Highway Practice, NCHRP Synthesis 298, Transportation Research Board, National Research Council, Washington, DC, p. 53, Table C-1C.

Habib, P.A. 1980. "Transportation System Management Options for Downtown Curbside Pickup and Delivery of Freight," Transportation Research Record 758, Transportation Research Board, National Research Council, Washington, DC, pp. 63-69.

Hogema, J.H., ARA Vanderhorst, and P.J. Bakker. 1994. Evaluation of the 16 Fog-Signaling System with Respect to Driving Behaviour (Evaluatie Van Het a 16 Mistsignaleringssysteem in Termen Van Het Rijgedrag). Report TNO-TM 1994 C-48. TNO Technische Menskunde, Soesterberg, Netherlands.

Ibrahim, A., and F.L. Hall. 1994. "Effect of Adverse Weather Conditions on Speed-Flow-Occupancy Relationships," Transportation Research Record 1457, Transportation Research Board, National Research Council, Washington DC, pp. 184-191.

Lamm, R., E.M. Choueiri, and T. Mailaender. 1990. "Comparison of Operating Speeds on Dry and Wet Pavements of Two-Lane Rural Highways," Transportation Research Record 1280, Transportation Research Board, National Research Council, Washington DC, pp. 199-207.

Metropolitan Transportation Authority. 2003. "Bridges \& Tunnels: Average Queue Waiting Time" (http://www.mta.nyc.ny.us/mta/ind-perform/month/bt-queue.htm).

Morris, A.G., A.L. Kornhauser, M.J. Kay. 1999. "Getting the Goods Delivered in Dense Urban Areas: A Snapshot of the Last Link of the Supply Chain," Transportation Research Record 1653, Transportation Research Board, National Research Council, Washington, DC, pp. 34-41.

(NCHRP) National Cooperative Highway Research Program. 1993. Electronic Toll and Traffic Management (ETTM) Systems - A Synthesis of Highway Practice, Transportation Research Board, National Research Council, Washington, DC.

Saka, A. A. 2002. Assessment of the Electronic Toll Collectionon Mobile Emissions in the Baltimore Metropolitan Area. Throughput Volumes, National Transportation Center, Baltimore, February, p. 10.

Schrank, D., and T. Lomax. 2004. 2004 Urban Mobility Study Data, (http://mobility.tamu.edu/ums/congestion data/tables/complete data.xls), Texas Transportation Institute (TTI), Texas A\&M University, College Station, Texas, accessed Oct. 1, 2004.

Skabardonis, A., T. Chira-Chavala, and D. Rydzewski. 1998. The I-880 Field Experiment: Effectiveness of Incident Detection Using Cellular Phones, California PATH Research Report UCB-ITS-PRR-98-1 Report for MOU 119, January.

(T.DOT) Tennessee Department of Transportation. Average Daily Traffic data, Nashville, Tennessee.

Traffic Technologies, Incorporated. 2001. United States Toll Facilities (http://www.ettm.com/).

(TRB) Transportation Research Board. 2000. Highway Capacity Manual, National Research Council, Washington, DC. 
(TRB) Transportation Research Board. 1985. Highway Capacity Manual, Special Report 209, National Research Council, Washington, DC.

U.S. Census Bureau. ZIP Code Business Patterns (C1-E99-ZCBP-09-US1)

(http://www.census.gov/mp/www/Tempcat/ZBP.html).

(U.S. DOL/BLS) U.S. Department of Labor, Bureau of Labor Statistics. State and County Employment and Wages from Covered Employment and Wages (http://www.bls.gov/cew/cewover.htm\#content).

(U.S. DOT/FHWA) U.S. Department of Transportation, Federal Highway Administration. 2001. Highway Statistics 2000 (http://www.fhwa.dot.gov/ohim/hs00/index.htm).

(U.S. DOT/FHWA) U.S. Department of Transportation, Federal Highway Administration. 2001. Toll Facilities in the United States: Bridges - Roads - Tunnels - Ferries, FHWA-PL-01-020, web-only publication (http://www.fhwa.dot.gov/ohim/tollpage.htm).

(U.S. DOT/FHWA) U.S. Department of Transportation, Federal Highway Administration. 2000. National Highway Planning Network (NHPN) Version 2.2, 1999 dataset (http://www.fhwa.dot.gov/hep10/data/nhpntoc.html).

(U.S. DOT/FHWA) U.S. Department of Transportation, Federal Highway Administration. 2000.

Highway Performance Monitoring System (HPMS), 1999 dataset

(http://www.fhwa.dot.gov/ohim/hpmspage.htm).

(U.S. DOT/FHWA) U.S. Department of Transportation, Federal Highway Administration. 2000. Highway Performance Monitoring System Field Manual (http://www.fhwa.dot.gov/ohim/hpmsmanl/appn.htm).

(U.S. DOT/FHWA) U.S. Department of Transportation, Federal Highway Administration. 2000.

Highway Statistics 1999 (http://www.fhwa.dot.gov/ohim/hs99/index.htm).

(U.S. DOT/FRA) U.S. Department of Transportation, Federal Railroad Administration, Office of Rail Safety. 2003. Highway-Rail Crossing Database Files (http://safetydata.fra.dot.gov/officeofsafety/Downloads/Default.asp).

(U.S. DOT/FRA) U.S. Department of Transportation, Federal Railroad Administration, Office of Policy and Program Development. 2002. GradeDec 2000 version 2.2: Reference Manual (http://www.fra.dot.gov/pdf/policy/Model.pdf).

(U.S. DOT/NHTSA) U.S. Department of Transportation, National Highway Traffic Safety Administration. Fatality Analysis Reporting System (FARS) database (http://wwwfars.nhtsa.dot.gov/main.cfm).

Vollmer Associated LLP. 2000. E-ZPass Evaluation Report: Travel Time Savings. New York Thruway Authority, August, pp. 32-33.

Wilbur Smith \& Associates. 2001. Operational and Traffic Benefits of E-ZPass to the New Jersey Turnpike. New Jersey Turnpike Authority, New Jersey, August, pp. 4-5. 